\title{
Evidence for Neutrino Oscillations in the Sudbury Neutrino Observatory \\ by
}

\author{
Alysia Diane Marino \\ A.B. (Princeton University) 1998 \\ M.A. (University of California, Berkeley) 2000 \\ A dissertation submitted in partial satisfaction of the \\ requirements for the degree of \\ Doctor of Philosophy \\ in \\ Physics \\ in the \\ GRADUATE DIVISION \\ of the \\ UNIVERSITY OF CALIFORNIA, BERKELEY
}

\author{
Committee in charge: \\ Dr. Kevin T. Lesko, Co-Chair \\ Professor Marjorie D. Shapiro, Co-Chair \\ Professor Kam-Biu Luk \\ Professor Heino Nitsche
}

Fall 2004 
The dissertation of Alysia Diane Marino is approved:

\begin{tabular}{lr}
\hline Co-Chair & Date \\
\hline Co-Chair & Date \\
\hline & \\
\hline & Date \\
\hline
\end{tabular}

University of California, Berkeley

Fall 2004 
Evidence for Neutrino Oscillations in the Sudbury Neutrino Observatory

Copyright 2004

by

Alysia Diane Marino 


\begin{abstract}
Evidence for Neutrino Oscillations in the Sudbury Neutrino Observatory by

Alysia Diane Marino

Doctor of Philosophy in Physics

University of California, Berkeley

Dr. Kevin T. Lesko, Co-Chair; Professor Marjorie D. Shapiro, Co-Chair
\end{abstract}

The Sudbury Neutrino Observatory (SNO) is a large-volume heavy water Čerenkov detector designed to resolve the solar neutrino problem. SNO observes charged-current interactions with electron neutrinos, neutral-current interactions with all active neutrinos, and elastic-scattering interactions primarily with electron neutrinos with some sensitivity to other flavors.

This dissertation presents an analysis of the solar neutrino flux observed in SNO in the second phase of operation, while $\sim 2$ tonnes of salt $(\mathrm{NaCl})$ were dissolved in the heavy water. The dataset here represents 391 live days of data. Only the events above a visible energy threshold of $5.5 \mathrm{MeV}$ and inside a fiducial volume within $550 \mathrm{~cm}$ of the center of the detector are studied. The neutrino flux observed via the charged-current interaction is $\left[1.71 \pm 0.065\right.$ (stat.) $\pm_{0.068}^{0.065}$ (sys.) \pm 0.02 (theor.) $] \times 10^{6} \mathrm{~cm}^{-2} \mathrm{~s}^{-1}$, via the elastic-scattering interaction is $\left[2.21 \pm 0.22\right.$ (stat.) $\pm_{0.12}^{0.11}$ (sys.) \pm 0.01 (theor.) $] \times 10^{6} \mathrm{~cm}^{-2} \mathrm{~s}^{-1}$, and via the neutral-current 
interaction is $\left[5.05 \pm 0.23\right.$ (stat.) $\pm_{0.37}^{0.31}$ (sys.) \pm 0.06 (theor.) $] \times 10^{6} \mathrm{~cm}^{-2} \mathrm{~s}^{-1}$. The electron-only flux seen via the charged-current interaction is more than $7 \sigma$ below the total active flux seen via the neutral-current interaction, providing strong evidence that neutrinos are undergoing flavor transformation as they travel from the core of the Sun to the Earth. The most likely origin of the flavor transformation is matter-induced flavor oscillation.

Dr. Kevin T. Lesko

Dissertation Committee Co-Chair

Professor Marjorie D. Shapiro Dissertation Committee Co-Chair 
To my parents and grandparents. 
With a grain of salt.

-Pliny the Elder, Natural History, Book 23, Section 8 


\section{Acronyms Used in this Dissertation}

AV: Acrylic Vessel; The Acrylic Vessel contains the 1000 metric tons of $\mathrm{D}_{2} \mathrm{O}$

CC: Charged-Current neutrino interaction with deuterium

ES: Elastic-Scattering neutrino interaction with electrons

FTG: The grid fitter, the reconstruction algorithm used in this dissertation, described in Chapter 3

MC: Monte Carlo simulated

m.w.e.: Meters Water Equivalent, used as a measure of the depth of an experiment, gives the equivalent depth of water that would provide the same mass of material above an experiment

NC: Neutral-Current neutrino interaction with deuterium

NCD: Neutral Current Detector, one of an array of ${ }^{3} \mathrm{He}$ proportional counters which has been inserted into the SNO detector to detect neutrons

OWL PMT: OutWard-Looking Phototube; There are 96 of these tubes looking into the Outer $\mathrm{H}_{2} \mathrm{O}$ (See Figure 2.1.)

PDF: probability density function

PMT: Photomultiplier tube 
PSUP: Pmt SUPport structure; the geodesic frame supporting the $\sim 10,000$ phototubes SNO: Sudbury Neutrino Observatory

SNOMAN: SNO Monte Carlo and ANalysis, the primary SNO software package

SNU: Solar Neutrino Unit, defined as $10^{-36} \mathrm{sec}^{-1}$ per target atom 


\section{Contents}

Glossary: Acronyms Used in this Dissertation iii

List of Figures $\quad$ ix

List of Tables $\quad$ xii

Preface: A Roadmap for this Dissertation $\quad$ xv

1 Introduction to the Solar Neutrino Problem 1

1.1 What is a Neutrino? . . . . . . . . . . . . . . . . . . . . 1

1.2 A Brief History of the Neutrino . . . . . . . . . . . . . . . 2

1.3 Solar Neutrinos . . . . . . . . . . . . . . . . . . 5

1.4 Solar Neutrino Experiments before SNO . . . . . . . . . . . . . 7

1.4.1 The Homestake Experiment . . . . . . . . . . . . . . . . 9

1.4.2 The Gallium Experiments . . . . . . . . . . . . . . . . . . . 9

1.4.3 Kamiokande and Super-Kamiokande . . . . . . . . . . . . . . . . 10

1.5 The Solar Neutrino Problem . . . . . . . . . . . . . . . . . . . . . . . . 11

1.6 Neutrino Oscillation . . . . . . . . . . . . . . . . . . . 13

1.6.1 Two-Flavor Oscillations . . . . . . . . . . . . . . . . 15

1.6.2 Oscillations in Matter . . . . . . . . . . . . . . . . . 15

1.6.3 Other Evidence for Neutrino Oscillation . . . . . . . . . . . . . . . . 17

1.7 Massive Neutrinos . . . . . . . . . . . . . . . . . . . . . . . 21

2 The Sudbury Neutrino Observatory $\quad 23$

2.1 The SNO Detector . . . . . . . . . . . . . . . . 23

2.1.1 The Heavy Water . . . . . . . . . . . . . . . . . . 25

2.1.2 The Acrylic Vessel . . . . . . . . . . . . . . . . . . 25

2.1 .3 The Light Water . . . . . . . . . . . . . . . . . . 27

2.1.4 The Photo-multiplier Tubes and PMT Support Structure . . . . . . 27

2.1.5 The Coordinate System . . . . . . . . . . . . . . . 28

2.1.6 Electronics and Data Acquisition . . . . . . . . . . . . . . . 30

2.1.7 Calibration System and Calibration sources . . . . . . . . . . . . 31 
2.2 Monte Carlo Simulation Package . . . . . . . . . . . . . . . . . . 34

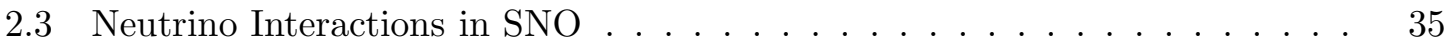

2.4 Previous Solar Neutrino Results from SNO . . . . . . . . . . . . . 38

3 Event Reconstruction $\quad 42$

3.1 Event Reconstruction Algorithm . . . . . . . . . . . . . . . . . . . 42

3.2 Reconstruction Spatial Performance . . . . . . . . . . . . . . . 45

3.3 Reconstruction Directional Performance . . . . . . . . . . . . . . . 52

3.4 Reconstruction Efficiency . . . . . . . . . . . . . . . . 54

3.5 Summary of Reconstruction . . . . . . . . . . . . . . . 56

4 Energy Calibration $\quad \mathbf{5 8}$

4.1 Energy Estimator based on ${ }^{16} \mathrm{~N}$ Source Calibration . . . . . . . . . . . 58

4.1.1 Number of hits on working PMTs: $n_{w h i t s} \ldots \ldots \ldots 58$

4.1.2 Noise and Tubes Online Correction . . . . . . . . . . . . . . . 59

4.1 .3 Date Correction . . . . . . . . . . . . . . . . 60

4.1 .4 Radial Correction . . . . . . . . . . . . . . . . 63

4.1.5 Number of effective PMT hits: $n_{\text {eff }} \ldots \ldots \ldots \ldots$. . . . . . . . 65

4.2 Verification of Energy Scale with ${ }^{252} \mathrm{Cf}$ and AmBe Sources . . . . . . . . . 66

4.3 Energy vs. Angle . . . . . . . . . . . . . . . . . . . . 69

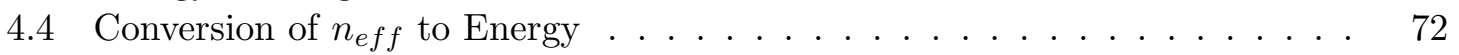

4.5 Energy Resolution . . . . . . . . . . . . . . . . . . . 73

4.6 Summary of Energy Uncertainty . . . . . . . . . . . . . . . 75

5 Event Isotropy $\quad \mathbf{7 6}$

5.1 Using Light Isotropy to Distinguish Neutrino Event Types . . . . . . . . . . 76

$5.2 \theta_{i j}$ - Measure of Isotropy . . . . . . . . . . . . . . . . . 77

5.3 Temporal Variation of $\theta_{i j} \ldots \ldots \ldots \ldots \ldots \ldots$

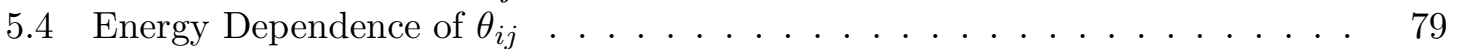

5.5 Radial Dependence of $\theta_{i j} \ldots \ldots \ldots \ldots \ldots$. . . . . . . . . . . . . . . . . . . . . . . 81

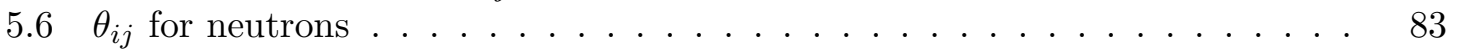

$5.7 \theta_{i j}$ for $4.4 \mathrm{MeV}$ Gammas from AmBe source . . . . . . . . . . . . 86

5.8 Summary of Uncertainties on $\theta_{i j} \ldots \ldots \ldots \ldots \ldots$

6 Neutron Detection Efficiency 90

6.1 Neutron Capture . . . . . . . . . . . . . . . . . . . . . . 90

6.2 The AmBe Source . . . . . . . . . . . . . . . . . . . . . . . 91

6.3 Determining the Number of $\gamma$-rays . . . . . . . . . . . . . . . . 93

6.4 Determining the Number of Coincidences . . . . . . . . . . . . . . . . 94

6.5 Efficiency vs Radius . . . . . . . . . . . . . . . . . . . . . . . 98

6.6 Summary of Neutron Detection Efficiency . . . . . . . . . . . . . . 101 
7 Removal of Non-Physics Events 103

7.1 Instrumental Backgrounds . . . . . . . . . . . . . . . . . . . . . 103

7.2 Cuts to Remove Instrumental Backgrounds . . . . . . . . . . . . . . 105

7.2.1 Level 1: Instrumental Background Cuts . . . . . . . . . . . . . . . 105

7.2.2 Level 2: High Level Cuts . . . . . . . . . . . . . . . . . . . . . 107

7.3 Purity of the Dataset . . . . . . . . . . . . . . . . . . 112

8 Physics Backgrounds $\quad \mathbf{1 1 5}$

8.1 Introduction to Physics Backgrounds . . . . . . . . . . . . . . . . . . 115

8.2 Internal $\mathrm{U}$ and $\mathrm{Th}$ Backgrounds . . . . . . . . . . . . . . . 116

8.2.1 ${ }^{238} \mathrm{U}$ and ${ }^{232} \mathrm{Th}$ Levels in Acrylic . . . . . . . . . . . . . . . . . 116

8.2.2 ${ }^{238} \mathrm{U}$ and ${ }^{232}$ Th Levels in $\mathrm{D}_{2} \mathrm{O}$ and $\mathrm{H}_{2} \mathrm{O} \ldots \ldots \ldots$

8.2.3 Summary of backgrounds from Internal U and Th . . . . . . . . . . 121

$8.3{ }^{238} \mathrm{U}$ and ${ }^{232} \mathrm{Th}$ in the PMTs and PSUP . . . . . . . . . . . . . . 123

8.4 External Gamma Rays . . . . . . . . . . . . . . . . . . . . . . 126

8.5 Muon-Induced Spallation _ . . . . . . . . . . . . . . . . . 130

8.5.1 Spallation on ${ }^{16} \mathrm{O} \ldots \ldots \ldots \ldots \ldots 131$

8.5.2 Spallation on $\mathrm{Cl}$ and $\mathrm{Na} \ldots \ldots \ldots \ldots . \ldots . \ldots . \ldots 134$

8.5.3 Spallation Neutrons . . . . . . . . . . . . . . . . . . . 134

8.5.4 Delayed Neutron-Capture Reactions . . . . . . . . . . . . . . . . 135

8.5.5 (n,p) Reactions and Muon Capture . . . . . . . . . . . . . . 136

8.5.6 Summary of Muon-Induced Backgrounds . . . . . . . . . . . . . . 138

8.6 Acrylic Vessel Events . . . . . . . . . . . . . . . . . . . . . . . . . . . 140

8.6.1 Description of the Events . . . . . . . . . . . . . . . . 140

8.6.2 Estimate of AV Event contamination . . . . . . . . . . . . . . . 145

8.7 Other Backgrounds . . . . . . . . . . . . . . . . . . . . 150

8.8 Summary of Backgrounds . . . . . . . . . . . . . . . 152

9 Signal Extraction $\quad 153$

9.1 Introduction . . . . . . . . . . . . . . . . . . 153

9.2 Maximum Likelihood Algorithm . . . . . . . . . . . . . . . . . 156

9.3 The PDFs . . . . . . . . . . . . . . . . . . . . 158

9.4 Performance of Extraction Algorithm on Monte Carlo Simulated Datasets . 159

9.5 The Dataset . . . . . . . . . . . . . . . . . . . . . . 160

9.6 Extraction on Data . . . . . . . . . . . . . . . . . . . . 161

9.7 Systematics . . . . . . . . . . . . . . . . . 164

10 Interpretation of Results and Conclusions $\quad 167$

10.1 Neutrino Fluxes . . . . . . . . . . . . . . . . . . 167

10.2 Interpretation of Fluxes . . . . . . . . . . . . . . . . 170

10.3 Neutrino Oscillation Parameters . . . . . . . . . . . . . . . . . 171

10.4 Conclusions . . . . . . . . . . . . . . . . . . . 175 
A KamLAND Electronics $\quad \mathbf{1 8 1}$

A.1 Waveform Digitization in the KamLAND Detector . . . . . . . . . . . . 181

A.2 ATWD Tester Setup . . . . . . . . . . . . . . . . . . . 183

A.3 Testing Criteria and Procedure . . . . . . . . . . . . . . . . 184

A.4 Testing Results . . . . . . . . . . . . . . . . . . . 187

$\begin{array}{lr}\text { Bibliography } & 189\end{array}$ 


\section{List of Figures}

1.1 The pp chain in the $\operatorname{Sun} \ldots \ldots \ldots \ldots \ldots$

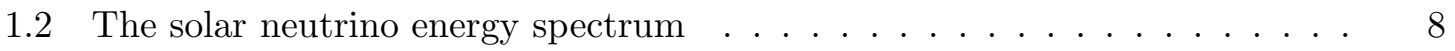

1.3 The solar sound speed . . . . . . . . . . . . . . . . . . . 12

1.4 Neutrino interactions with electrons . . . . . . . . . . . . . 16

1.5 The allowed neutrino oscillation parameters. . . . . . . . . . . . 18

2.1 A cross section of the SNO Detector. . . . . . . . . . . . . . 24

2.2 The SNO acrylic vessel which contains the $\mathrm{D}_{2} \mathrm{O} \ldots \ldots \ldots \ldots$

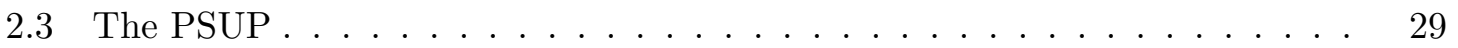

2.4 The source manipulator system . . . . . . . . . . . . . . . . 32

2.5 A view of the SNO acrylic vessel with the NCDs. . . . . . . . . . . 39

2.6 Flux of electron and non-electron ${ }^{8} \mathrm{~B}$ solar neutrinos from SNO . . . . . . 41

3.1 Time Residual distribution for fitter. . . . . . . . . . . . . . . . . 44

3.2 Angular distribution for fitter. . . . . . . . . . . . . . 46

3.3 Fit Deviation for ${ }^{16} \mathrm{~N} \ldots \ldots \ldots \ldots \ldots$. . . . . . . . . . . 47

3.4 Fit Deviation for ${ }^{252} \mathrm{Cf} \ldots \ldots \ldots \ldots \ldots$

3.5 Diagram of fitter pull . . . . . . . . . . . . . . . . . . 49

3.6 Fitter radial pull for ${ }^{16} \mathrm{~N}$ source . . . . . . . . . . . . . . . . . 50

3.7 Fitter radial pull near edge of $\mathrm{D}_{2} \mathrm{O}$ for ${ }^{16} \mathrm{~N}$ source . . . . . . . . . . . . 51

3.8 Fitter radial pull for electron and neutron $\mathrm{MC} \ldots \ldots \ldots$. . . . . . . . . . . . . . . . 52

3.9 Schematic of determining angular resolution with ${ }^{16} \mathrm{~N}$ source . . . . . . . 53

3.10 Angular resolution vs. ${ }^{16} \mathrm{~N}$ source radial position . . . . . . . . . . . . 54

3.11 Angular resolution for ${ }^{16} \mathrm{~N}$ source data and $\mathrm{MC} \ldots \ldots \ldots \ldots$

3.12 Reconstruction Efficiency . . . . . . . . . . . . . . . 56

$4.1 n_{\text {whits }}$ for a single ${ }^{16} \mathrm{~N}$ run $\ldots \ldots \ldots \ldots \ldots \ldots$

4.2 Noise and Online Corrected ${ }^{16} \mathrm{~N}$ Data at Center . . . . . . . . . . . . . . . 62

4.3 Noise and Online Corrected ${ }^{16} \mathrm{~N}$ MC at Center . . . . . . . . . . . . . . . . . 63

4.4 Noise, Online, and tcor Corrected ${ }^{16} \mathrm{~N}$ Data and MC . . . . . . . . . . . . 64

4.5 Noise, Online, and tcor Corrected ${ }^{16} \mathrm{~N}$ Data and MC vs. Radius . . . . . 65

4.6 Mean $n_{\text {eff for }}{ }^{16} \mathrm{~N}$ Data and MC vs. Radius . . . . . . . . . . . . . . 66 
$4.7 n_{\text {eff for }}{ }^{252} \mathrm{Cf}$ Data and $\mathrm{MC}$ vs. Radius . . . . . . . . . . . . . . . . 68

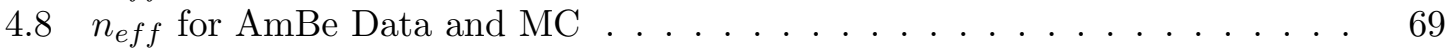

$4.9 n_{\text {eff }}$ vs. direction for ${ }^{16} \mathrm{~N}$ Data and $\mathrm{MC} \ldots \ldots \ldots \ldots$

$4.10 n_{\text {eff }}$ vs. direction for ${ }^{252} \mathrm{Cf}$ Data and $\mathrm{MC} \ldots \ldots \ldots . \ldots \ldots 71$

4.11 Total Energy vs. $n_{\text {eff }} \ldots \ldots \ldots \ldots \ldots \ldots$. . . . . . . . . . . . . . . . . . . 73

4.12 Width of $n_{\text {eff }}$ for ${ }^{16} \mathrm{~N}$ Data and MC vs. Radius . . . . . . . . . . . . . 74

4.13 Width of $n_{\text {eff }}$ for ${ }^{252} \mathrm{Cf}$ Data and $\mathrm{MC}$ vs. Radius . . . . . . . . . . . . . 74

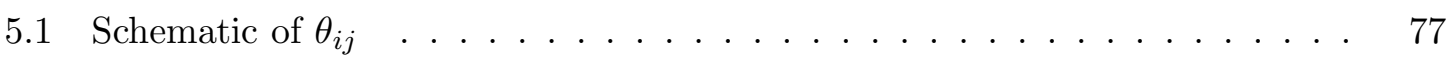

5.2 Example of a $\theta_{i j}$ distribution for electrons . . . . . . . . . . . . 78

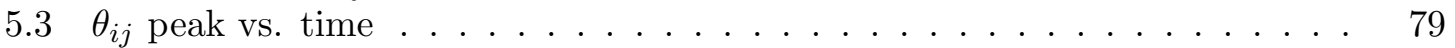

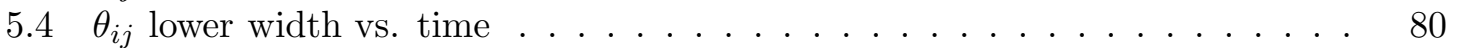

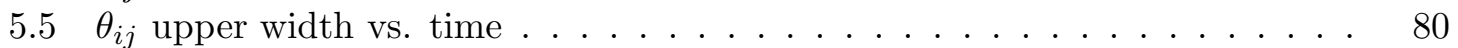

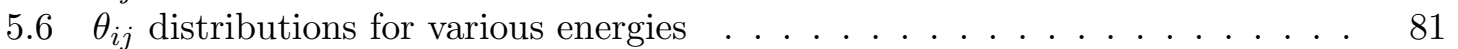

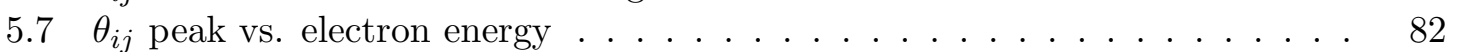

$5.8 \theta_{i j}$ lower width vs. electron energy . . . . . . . . . . . . . . . . . . . 82

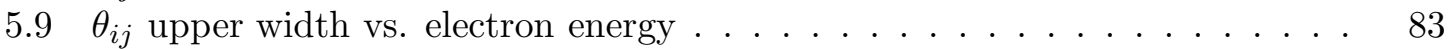

$5.10 \theta_{i j}$ peak vs. reconstructed position for ${ }^{16} \mathrm{~N} \ldots \ldots \ldots \ldots$. . . . . 84

$5.11 \theta_{i j}$ lower width vs. reconstructed position for ${ }^{16} \mathrm{~N} \ldots \ldots \ldots$. . . . . . 84

$5.12 \theta_{i j}$ upper width vs. reconstructed position for ${ }^{16} \mathrm{~N} \ldots \ldots \ldots$

5.13 Example of a $\theta_{i j}$ distribution for neutrons $\ldots \ldots \ldots \ldots$

$5.14 \theta_{i j}$ peak vs. reconstructed position for ${ }^{252} \mathrm{Cf} \ldots \ldots \ldots \ldots$

$5.15 \theta_{i j}$ lower width vs. reconstructed position for ${ }^{252} \mathrm{Cf} \ldots \ldots \ldots$. . . . . . 87

$5.16 \theta_{i j}$ upper width vs. reconstructed position for ${ }^{252} \mathrm{Cf} \ldots \ldots \ldots$

$5.17 \theta_{i j}$ distribution for $\gamma$-rays from AmBe source . . . . . . . . . . . . 89

6.1 Decay scheme of AmBe source . . . . . . . . . . . . . . . . . . 92

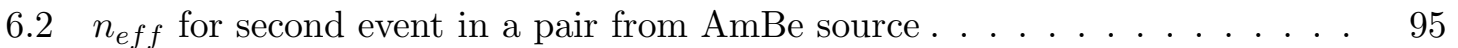

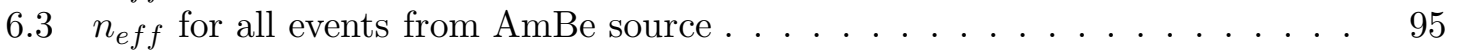

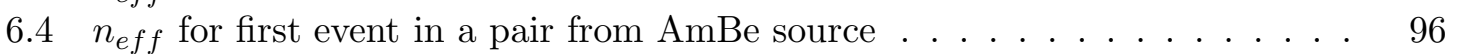

6.5 Neutron times for events from AmBe source . . . . . . . . . . . . . . 97

6.6 Time to next event from AmBe source data . . . . . . . . . . . . . . . . . . 99

6.7 AmBe Efficiency vs. Radius . . . . . . . . . . . . . . . . . . . . . . 100

$7.1{ }^{16} \mathrm{~N}$ source Level 2 Cut acceptance vs. Time . . . . . . . . . . . . . . . . . 109

$7.2{ }^{16} \mathrm{~N}$ Data Level 2 Cut acceptance vs. R . . . . . . . . . . . . . . . . 109

$7.3{ }^{16} \mathrm{~N}$ MC Level 2 Cut acceptance vs. R . . . . . . . . . . . . . . 110

$7.4{ }^{252}$ Cf Data Level 2 Cut acceptance vs. R . . . . . . . . . . . . . . . . . 111

$7.5{ }^{252} \mathrm{Cf}$ MC Level 2 Cut acceptance vs. R . . . . . . . . . . . . . . . 111

7.6 Region in Bifurcated-Analysis . . . . . . . . . . . . . . . . . . . . 114

$8.1{ }^{232}$ Th Decay Chain . . . . . . . . . . . . . . . . . . . . . . . . . . . . . . . . .

$8.2{ }^{238} \mathrm{U}$ Decay Chain . . . . . . . . . . . . . . . . . . . . 118

$8.3{ }^{208} \mathrm{Tl}$ and ${ }^{214} \mathrm{Bi}$ Decay Schemes . . . . . . . . . . . . . . . . . . 119

8.4 Schematic of external $\gamma$-ray analysis f . . . . . . . . . . . . . . 128 


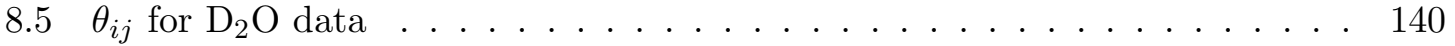

$8.6 \theta_{i j}$ for $\mathrm{D}_{2} \mathrm{O}$ data with ITR cut applied . . . . . . . . . . . . 141

8.7 Side View 1 of AV Events . . . . . . . . . . . . . . . . . . . . . . 142

8.8 Side View 2 of AV Events . . . . . . . . . . . . . . . . . . . . 143

8.9 Top view of AV Events . . . . . . . . . . . . . . . . . . . . . . . . . . . . . . . . 144

8.10 AV events vs. Time of Day . . . . . . . . . . . . . . . . 146

8.11 AV events vs. Time . . . . . . . . . . . . . . . . . 146

8.12 Fit for "AV" Events within $550 \mathrm{~cm} \ldots \ldots \ldots \ldots$. . . . . . . . 148

8.13 Fit for "AV" Events within $600 \mathrm{~cm} \ldots \ldots \ldots \ldots$

9.1 Definition of $\cos \left(\theta_{\text {sun }}\right) \ldots \ldots \ldots \ldots \ldots \ldots \ldots \ldots$

9.2 Predicted neutrino E, R, $\cos \left(\theta_{\text {sun }}\right)$, and $\theta_{i j}$ distributions $\ldots \ldots \ldots \ldots$

$9.3 \cos \left(\theta_{\text {sun }}\right)$ Distribution of Data and Fit . . . . . . . . . . . . . . . . . . 162

$9.4 \mathrm{R}_{f i t}$ Distribution of Data and Fit . . . . . . . . . . . . . 162

$9.5 \theta_{i j}$ Distribution of Data and Fit . . . . . . . . . . . . . . 163

$9.6 n_{\text {eff }}$ Distribution of Data and Fit . . . . . . . . . . . . . 163

10.1 Total Flux vs. Electron Neutrino Flux . . . . . . . . . . . . . . . . . . 172

10.2 Schematic of oscillation contour generation procedure . . . . . . . . . . 174

10.3 Allowed oscillation contours for SNO salt flux results . . . . . . . . . . . 176

10.4 Allowed oscillation contours for $\mathrm{SNO}$ salt $+\mathrm{D}_{2} \mathrm{O}$ flux results $\ldots \ldots \ldots$. . . 177

A.1 The KamLAND detector . . . . . . . . . . . . . . . . . . . . . . 182

A.2 Sample Waveform from KamLAND ATWD . . . . . . . . . . . . . . . 184 


\section{List of Tables}

1.1 Results from previous solar neutrino experiments . . . . . . . . . . . 11

3.1 Summary of Reconstruction Systematics . . . . . . . . . . . . . . 57

4.1 Summary of Energy systematics _ . . . . . . . . . . . . . 75

5.1 Summary of Isotropy systematics . . . . . . . . . . . . . . . . 89

6.1 Neutron Detection Efficiency inside $725 \mathrm{~cm}$ and above $n_{\text {eff }}=24 \ldots$. . . 101

6.2 Neutron Detection Efficiency inside $550 \mathrm{~cm}$ and above $5.5 \mathrm{MeV}$. . . . . . 102

7.1 Instrumental Cut Acceptance . . . . . . . . . . . . . . . . . . . . 107

7.2 Level 2 Cut Acceptance . . . . . . . . . . . . . . . . . . . . . . . . . . . . . . . . . . . .

7.3 Instrumental background contamination in the dataset . . . . . . . . . . . . 114

8.1 AV Radioactivity Concentrations . . . . . . . . . . . . . . . . . . . 120

$8.2 \mathrm{D}_{2} \mathrm{O}$ and $\mathrm{H}_{2} \mathrm{O}$ Radioactivity Concentrations . . . . . . . . . . . . . 121

8.3 Summary of Čerenkov backgrounds from $\mathrm{U}$ and $\mathrm{Th}$. . . . . . . . . . . . . . 122

8.4 Summary of neutron backgrounds from $\mathrm{U}$ and $\mathrm{Th}$ in $\mathrm{D}_{2} \mathrm{O} \ldots \ldots \ldots$

8.5 PMT radioactivity . . . . . . . . . . . . . . . . . . . . 124

8.6 Summary of backgrounds from $\mathrm{U}$ and Th in PMTs . . . . . . . . . . . . 125

8.7 External $\gamma$-ray contamination . . . . . . . . . . . . . . . . . . . . 129

8.8 Systematics on external $\gamma$-ray Contamination . . . . . . . . . . . . . . . 130

8.9 Possible Spallation Products in Salt Phase . . . . . . . . . . . . . . . . . 132

8.10 Neutron-capture processes . . . . . . . . . . . . . . . . . . . . 135

$8.11(\mathrm{n}, \mathrm{p})$ or $\mu^{-}$capture processes $\ldots \ldots \ldots \ldots \ldots$

8.12 Muon Background Summary . . . . . . . . . . . . . . . . . . 139

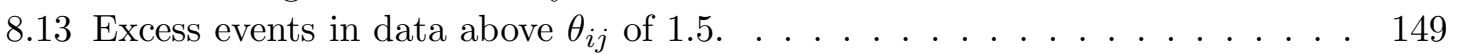

8.14 Systematic studies for "AV" event estimate . . . . . . . . . . . . . . . 149

8.15 Additional Backgrounds . . . . . . . . . . . . . . . . . . 151

8.16 Background Summary _. . . . . . . . . . . . . . . . . . 152

9.1 Signal extraction on $100 \mathrm{MC}$ test sets $\ldots \ldots \ldots \ldots$ 
9.2 Signal extraction results on Data . . . . . . . . . . . . . . . . . 161

9.3 Signal extraction correlation coefficients . . . . . . . . . . . . . . . 161

9.4 Uncertainties on Fit Results . . . . . . . . . . . . . . . . . . . . 165

10.1 Corrections to Flux Conversion _ . . . . . . . . . . . . . . . . 169

10.2 Flux Conversion . . . . . . . . . . . . . . . . . 170

10.3 Flux Results . . . . . . . . . . . . . . . . . . . 170

A.1 ATWD failure mode summary f . . . . . . . . . . . . . . . . 188 


\section{Acknowledgments}

First and foremost, I gratefully acknowledge the support of the U.S. Department of Energy, under contract No. DE-AC03-76SF00098, which has made this work possible.

Huge thanks my advisors, Kevin Lesko and Marjorie Shapiro. A special thank you to Alan Poon, Colin Okada, and Charles Currat, who at one time or another have put up with me as an officemate and provided much guidance and practical advice (and sometimes lots of chocolate). My fellow LBNL SNO colleagues Yuen-dat Chan, Reyco Henning, Xin Chen, Rick Norman, and Bob Stokstad provided much nuclear physics knowledge and put up with me as an apartmentmate in Sudbury.

Thanks to Stuart Freedman for allowing me the opportunity to get some hardware experience on KamLAND. Thanks also to Fred, Leo, Harold, Thorsten, Sanshiro, and Bruce Berger for their help with setting up the KamLAND chip tester and of course thanks to all of the chip testers. Throughout my time at LBL, both the library and NERSC were indispensable. Karen Edwards and Jeanne Miller ensured that all of those trips to Sudbury went smoothly.

Thanks to the 2003 AL West Division Champion Oakland A's. While all of those baseball games probably didn’t help me graduate any faster, they sure did make life more entertaining. My fellow INPA graduate students helped to keep lunchtime and teatime amusing. Above all, thanks to my family, my roommates, and Alex for keeping me (mostly) sane and going to baseball games with me. 


\section{Preface: A Roadmap for this}

\section{Dissertation}

I am a firm believer in the importance of having a map to get where one is going. To that aim, before we begin I would like to say a few words on the outline of this dissertation.

Chapter 1 begins with an account of the history of the neutrino and lays out the solar neutrino problem, which has been an outstanding mystery in particle physics for the past four decades. To put it simply, too few neutrinos from the Sun are observed at the Earth. With the Sudbury Neutrino Observatory (SNO), described in Chapter 2, this mystery has finally be solved. Previous solar neutrino experiments have been primarily sensitive to only one of the three types of neutrino, the electron neutrino. With its unique heavy water target, SNO can see all three kinds of neutrinos. If the flux of electron neutrinos from the Sun is measured to be less than the total neutrino flux, then this is evidence that neutrinos are undergoing flavor changes. This gives a possible resolution to the solar neutrino problem, and also strongly suggests that at least some neutrino flavors have mass.

The goal of this dissertation is to determine the flux of solar neutrinos on the Earth via various neutrino interactions. Unfortunately, this is not as simple as just counting up 
the number of observed neutrinos. There are many backgrounds in the detector, and it is not possible to distinguish the different neutrino interactions from one another on an event-by-event basis. Instead we must sum together many neutrino events and look at the resulting distributions to tell how many neutrinos of each type were present.

The first piece of information we need to know is where is the event located in the detector. We would also like to know which direction the event is traveling in. Both of these are treated in Chapter 3, which describes the event reconstruction within the detector.

Secondly, we need to know the energy of the event. We need to apply an energy cut to the data to reduce the low-energy backgrounds coming from radioactivity in the detector components. Chapter 4 will treat the energy calibration of the detector.

To help distinguish one type of neutrino interaction from another we can look at the isotropy of the pattern of light seen in the detector. Chapter 5 will describe how the isotropy of an event is quantified and will look at the calibration of the isotropy of the detector.

With the reconstruction, energy and isotropy of an event determined, all of the basics building blocks for the analysis are in place. Next we need to understand the backgrounds in the data and the efficiency of the detector. Chapter 6 explores the neutron detection efficiency of the detector. Chapter 7 deals with the non-physics backgrounds (electronics noise, etc.) and estimates their contribution to the final dataset. Chapter 8 estimates the backgrounds in the dataset from physics interactions, such as low-energy radioactive decays and muons.

In Chapter 9 the scheme for extracting the neutrino fluxes from the data is pre- 
sented. In Chapter 10 all of this information is put together to extract neutrino fluxes from the data, which are interpreted in a two-neutrino oscillation framework.

The Appendix describes work that was performed by the author on the electronics for the KamLAND reactor neutrino experiment.

Enjoy! 


\section{Chapter 1}

\section{Introduction to the Solar Neutrino}

\section{Problem}

\subsection{What is a Neutrino?}

Neutrinos are elusive particles. They have no electric charge and a very small mass relative to the other known particles. Though abundant in nature, neutrinos interact with other matter very weakly, making them extremely difficult to detect. It has been pointed out that a $50 \mathrm{MeV}$ neutrino can easily penetrate a piece of solid lead a light year thick without undergoing an interaction [1]. We believe that neutrinos are one of the fundamental building blocks of nature. Our present understanding ${ }^{1}$ is that all matter is composed of two types of particles: quarks and leptons. Quarks combine to form protons and neutrons and other heavy particles, while leptons are divided into charged leptons (such as electrons) and

\footnotetext{
${ }^{1}$ For any poor soul who stumbles across this dissertation 100 years from now and is convulsed with laughter while reading this paragraph, the author wishes to remind you that the present understanding is, of course, subject to change.
} 
neutrinos. At this point, it will be useful embark upon a brief historical digression to more fully explain the present picture of the neutrino.

\subsection{A Brief History of the Neutrino}

The story of the neutrino (denoted by the Greek letter $\nu$ ) begins in the 1930s. At that time, atomic nuclei were believed to consist of electrons and protons (with the mass number of protons and the mass number minus the atomic number of electrons in the nucleus) [2]. One of the difficulties of this model concerned nuclear $\beta$ decays, which had been observed to emit electrons with a broad spectrum of energies. As $\beta$ decay was believed to involve the ejection of one of the electrons from the nucleus, a discrete electron energy should have been observed due to the discrete quantum states in the nucleus and the fact that only two particles were present in the final state. Pauli first proposed a way out of this crisis in his famous letter of 1930 to the "radioactive ladies and gentleman" at a physics meeting in Tübingen. ${ }^{2}$ In this brief letter (an English translation of which can be found in [2]), Pauli hypothesized "the possibility that there could exist in the nuclei electrically neutral particles that I wish to call neutrons, which have spin $1 / 2$ and obey the exclusion principle." He went on to note that these particles are emitted along with the electron in $\beta$ decay -making it a 3-body final state- and that their mass must be less than 0.01 times the proton mass.

In 1932 Chadwick discovered the particles that we now call neutrons, but they were too heavy to satisfy Pauli's explanation of energy conservation in $\beta$ decay. In 1934

\footnotetext{
${ }^{2}$ Pauli elected not to attend this meeting due to a ball in Zürich.
} 
Fermi published in Italian [3] and German [4] his theory of $\beta$ decay (an English translation appears in [5]), in which he assumed the existence of Pauli's light neutral particle, which he called the neutrino. During a $\beta$ decay, a neutron is converted into a proton inside the nucleus, and an electron and a neutrino (really an anti-neutrino) are emitted.

In the late 1930s and 1940s several cloud chamber experiments studying nuclear recoil in $\beta$ decay further illustrated the need for these extra particles to conserve energy and momentum in decays, thus hinting at the existence of the neutrino $[6,7,8]$. The first direct detection of neutrinos occurred at the Savannah River nuclear reactor in 1956 [9]. Here Reines and Cowan and their group used a Cadmium-doped water target detecting the inverse- $\beta$ decay induced by anti-neutrinos coming from the reactor. This reaction $(\bar{\nu}+\mathrm{p}$ $\left.\rightarrow n+e^{+}\right)$produced a positron, detected by the two $0.511 \mathrm{MeV}$ gamma rays produced in positron/electron annihilation, and a neutron, which would capture on Cadmium a few $\mu$ sec later and emit gamma rays.

In 1956 Ray Davis and others [10] looked for the production of electrons by antineutrinos coming from the Savannah River Reactor using $\mathrm{Cl}$ as the target $\left(\bar{\nu}+n \rightarrow p+e^{-}\right)$. If neutrinos and anti-neutrinos are not distinct particles then this reaction, which is possible for neutrinos, should also occur for the anti-neutrinos coming from the reactor [1]. ${ }^{3}$ The work of the Davis group set an upper limit on the reaction rate [10] that was well below what would have been expected if neutrinos and anti-neutrinos are identical. In present terminology, we would say that $e^{-}, \mu^{-}, \tau^{-}$and $\nu$ have been assigned a lepton number of +1 , with $e^{+}, \mu^{+}, \tau^{+}$and $\bar{\nu}$ having a lepton number of -1 . It is believed that the total

\footnotetext{
${ }^{3}$ This neutrino interaction on $\mathrm{Cl}$ would go on to play a very important role in the detection of solar neutrinos.
} 
lepton number must be conserved in any standard electroweak interaction. For example, the $\bar{\nu}+n \rightarrow p+e^{-}$has a lepton number of -1 on the left side and +1 on the right side, so it is forbidden. However, $\nu+n \rightarrow p+e^{-}$is allowed since it has a lepton number of +1 on both sides.

In $1957 \mathrm{C} . \mathrm{S} . \mathrm{Wu}$ and others showed using polarized ${ }^{60}$ Co that $\beta$ decay violates parity [11], with the electron almost always traveling in the direction opposite the nuclear spin. In other words the angular distribution of the emitted $\beta \mathrm{s}$ is not symmetric with respect to the spin of the decaying nucleus. This was interpreted by Lee and Yang [12] into the two-component theory of the neutrino. They proposed that perhaps the neutrino spin always has the same orientation with respect to the neutrino momentum. This theory requires the neutrinos to be massless, since if a neutrino has mass, it must be traveling slower than the speed of light, making it possible to boost to a frame of reference where the neutrino spin will take on the opposite orientation with respect to the momentum. In 1959 an experiment performed by M. Goldhaber and others on ${ }_{63}^{152} \mathrm{Eu}[13]$ investigated the nature of this fixed orientation. It was revealed that the neutrino is "left-handed". Anti-neutrinos, which have the opposite spin orientation, are "right-handed".

All of the work discussed so far focussed on neutrinos and anti-neutrinos in interactions involving electrons. In 1962 it was observed that the neutrinos produced in pion decay will produce muons, not electrons, when they interact with matter [14]. This indicated that there are at least two families of neutrinos, electron neutrinos and muon neutrinos. When the $\tau$ lepton was discovered in the mid-1970s [15], the existence of a $\tau$ neutrino was quickly inferred. Interactions of $\nu_{\tau}$ were first observed in 2001 [16]. At the present moment we 
believe that leptons, like quarks, are divided into three doublets [17]:

$$
\left(\begin{array}{c}
e \\
\nu_{e}
\end{array}\right)\left(\begin{array}{c}
\mu \\
\nu_{\mu}
\end{array}\right)\left(\begin{array}{c}
\tau \\
\nu_{\tau}
\end{array}\right)
$$

Each charged lepton has a corresponding electrically-neutral neutrino, for a total of three neutrino flavors: electron $(\mathrm{e}), \mathrm{mu}(\mu)$ and tau $(\tau)$. Further support for the number of neutrino flavors comes from $\mathrm{e}^{+} \mathrm{e}^{-}$collider experiments. By studying the decay modes of the $\mathrm{Z}$ boson, the combined results from the 4 LEP experiments (ALEPH, DELPHI, L3, and OPAL) indicate that there are $2.984 \pm 0.008$ neutrino flavors which couple to the $\mathrm{Z}[18]$.

The mass of the neutrino will become very important later in this chapter and obtaining a clearer understanding of neutrino masses is one of the primary motivations for the work described in this dissertation. The masses have not yet been directly measured. The present limit on the electron neutrino mass is $<225 \mathrm{eV}$ (determined from the electron capture decay of ${ }^{163} \mathrm{Ho}$ ) and on the electron anti-neutrino mass is $<3 \mathrm{eV}$ (determined from ${ }^{3} \mathrm{H} \beta$ decay) [18]. The upper limits on the $\nu_{\mu}$ and $\nu_{\tau}$ masses are $0.19 \mathrm{MeV}$ and $18.2 \mathrm{MeV}$ respectively [18]. Currently there are no direct limits on on the $\bar{\nu}_{\mu}$ and $\bar{\nu}_{\tau}$ masses. ${ }^{4}$

\subsection{Solar Neutrinos}

Though neutrinos are difficult to detect and to study, they are quite abundant in nature. For example the average banana emits roughly 1.2 million neutrinos per day due to the decay of radioactive ${ }^{40} \mathrm{~K} .{ }^{5}$ More significant terrestrial sources include nuclear reactors and the decays of cosmic-ray muons and pions in the atmosphere. Neutrinos are

\footnotetext{
${ }^{4}$ The $\nu$ and $\bar{\nu}$ masses are not necessarily equal if CPT symmetry is violated.

${ }^{5}$ The average banana contains $467 \mathrm{mg}$ of potassium [19], which is $0.0117 \%{ }^{40} \mathrm{~K}$ in nature [20]. With a half-life of 1.3 billion years, ${ }^{40} \mathrm{~K}$ can decay by either $\beta^{+}$or $\beta^{-}$, producing both neutrinos and anti-neutrinos.
} 
also produced in great numbers in a core collapse supernova, where the core of a massive star explodes and forms a neutron star, releasing on the order of $10^{58}$ neutrinos [21]. Perhaps the largest set of neutrinos in the universe is the relic neutrinos left over from the Big Bang. These neutrinos and anti-neutrinos have a number density of 100 per $\mathrm{cm}^{3}$ per neutrino flavor. However, since they have an average temperature of $1.9 \mathrm{~K}(0.25 \mathrm{eV})$, they will be extremely difficult to observe directly [22].

The Sun is the most significant source of neutrinos within our solar system. A hydrogen-buring main sequence star, the Sun is fueled by nuclear fusion reactions occurring within the solar core (inner $\sim 20 \%$ of the Sun's radius). The principal fusion process in the Sun, called the p-p chain, involves 11 separate reactions, which can be summarized by the overall reaction $4 p \rightarrow \alpha+2 e^{+}+2 \nu_{e}$ [23]. Figure 1.1 shows the full p-p chain of interactions [23]. It should be emphasized that all of these reactions produce only electron neutrinos. The neutrinos produced by the two-proton fusion reaction are referred to as pp neutrinos, those from the interaction involving ${ }^{3} \mathrm{He}$ and a proton are called hep neutrinos, and those from the two-proton-and-electron reaction are known as pep neutrinos. The remaining neutrinos are typically labelled by the initial nuclei in the reactions where neutrinos are produced. The neutrinos produced by the various $\mathrm{p}-\mathrm{p}$ chain interactions have different neutrino energies. Figure 1.2 shows the energy spectra for the various neutrino interactions. Note that above $5 \mathrm{MeV}$ only neutrinos from the ${ }^{8} \mathrm{~B}$ and hep reactions are produced in significant amounts.

The study of solar neutrinos is important because it provides a window into the interior of the Sun. The photons which we see from the Sun emerge from only the outer 


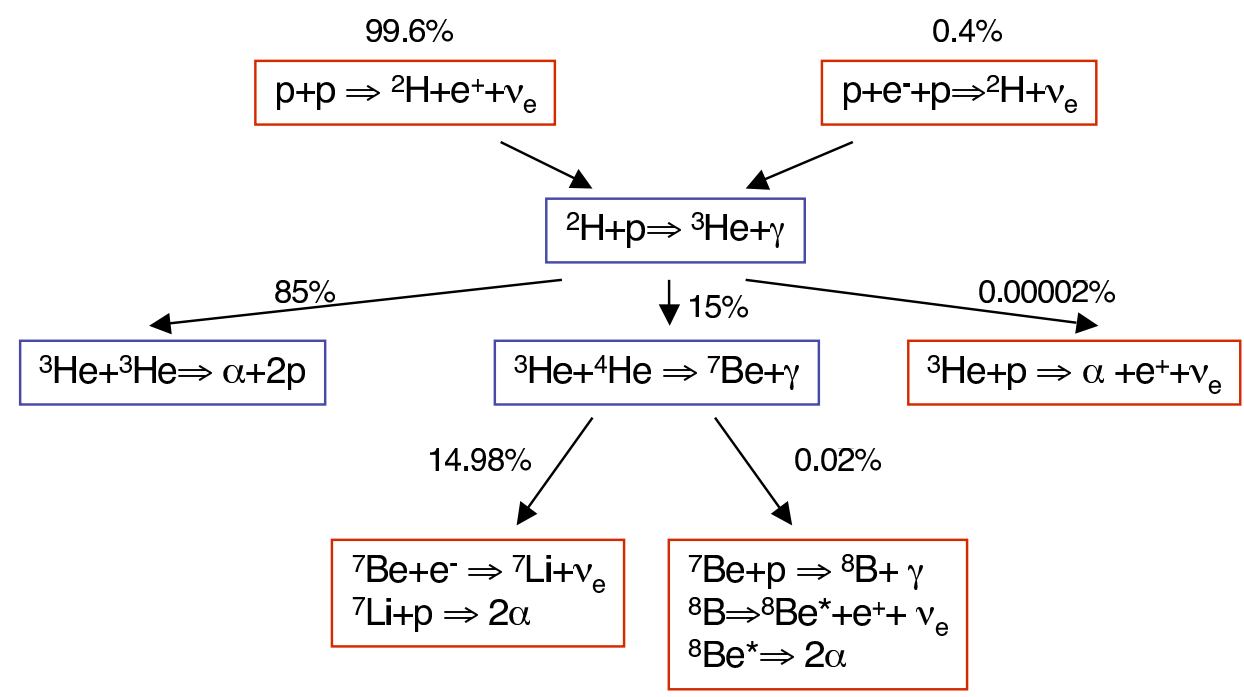

Figure 1.1: The individual fusion reactions that make up the pp chain in the core of the Sun $[23]$.

$0.1 \%$ of the Sun's radius [25]. Since neutrinos have such small interaction cross sections, they stream out of the solar core essentially undisturbed. Detailed measurements of the solar neutrino flux probe the nuclear fusion reactions fueling the Sun. Due to the large distance scales involved, solar neutrinos also provide a useful tool for the study of neutrino properties.

\subsection{Solar Neutrino Experiments before SNO}

In the late $1950 \mathrm{~s}$ it was realized that the decay of ${ }^{8} \mathrm{~B}$ in Figure 1.1 would provide a source of high-energy neutrinos from the Sun [26] and attempts were made to estimate the flux at the Earth [26, 27]. Experimenters began searching for methods of detecting these neutrinos in the 1960s. The first attempt to detect solar neutrinos was made by Ray Davis, using ${ }^{37} \mathrm{Cl}$ as a target. The experiment was located 2300 feet below the surface in a 


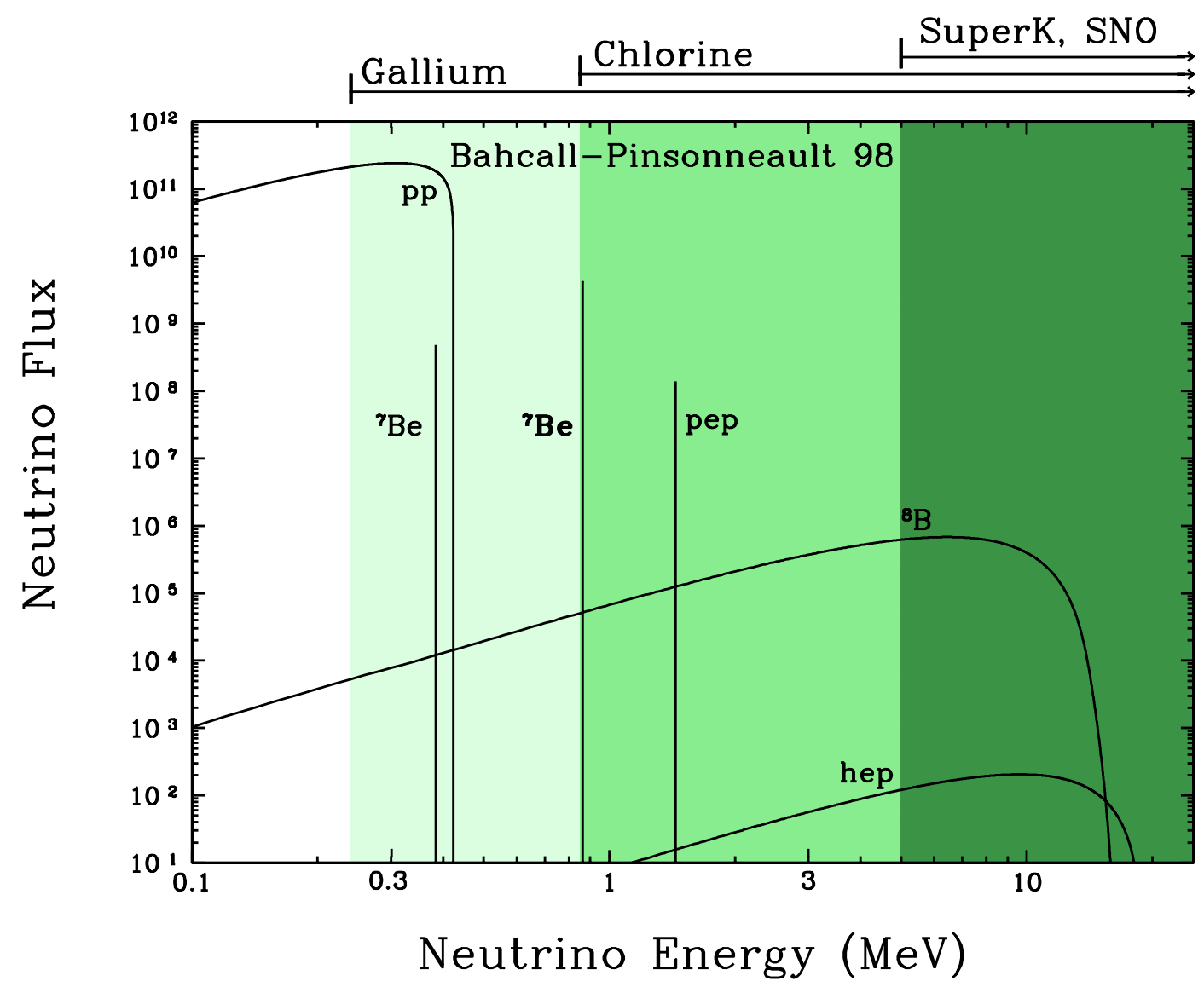

Figure 1.2: This figure, from John Bahcall, is an update of one in [24]. The shaded regions indicate the approximate energy thresholds for various neutrino detectors. 
limestone mine in Barbeton, Ohio, and only an upper limit could be obtained due to the cosmic-ray muon backgrounds [28], emphasizing the great importance that deep locations have in the study of solar neutrinos.

\subsubsection{The Homestake Experiment}

Continuing on with the work that had started in the Barbeton mine, Ray Davis and his group constructed a detector in the Homestake gold mine in Lead, South Dakota at a depth of $4850 \mathrm{ft}$ (4400 m.w.e.) [29]. The experiment used a radiochemical assay technique based on the reaction

$$
\nu_{e}+{ }^{37} \mathrm{Cl} \rightarrow{ }^{37} \mathrm{Ar}+e^{-}
$$

Using a large tank of cleaning fluid as the target, the ${ }^{37} \mathrm{Ar}$ produced by neutrino interactions was then extracted by purging the tank with helium, collecting the He and Ar. With a 35day half-life, the ${ }^{37} \mathrm{Ar}$ could then be counted. This experiment took data for over 30 years. The observed solar neutrino rate was $2.56 \pm 0.16$ (stat.) \pm 0.16 (syst.) SNU $^{6}$ [30], compared to a predicted rate of $7.7_{-1.0}^{+1.2} \mathrm{SNU}[31]$.

\subsubsection{The Gallium Experiments}

Another reaction which has been used to study solar neutrinos is

$$
\nu_{e}+{ }^{71} \mathrm{Ga} \rightarrow{ }^{71} \mathrm{Ge}+e^{-}
$$

The threshold for this reaction is $0.233 \mathrm{MeV}$, so it is sensitive to neutrinos coming from the pp reaction. There have been two approaches to using gallium to detect solar neutrinos,

\footnotetext{
${ }^{6} \mathrm{~A}$ SNU, or Solar Neutrino Unit, is defined as $10^{-36} \mathrm{sec}^{-1}$ per target atom.
} 
one using gallium metal (the SAGE experiment) and one using a gallium chloride solution (the GALLEX experiment, continued in the GNO experiment). SAGE, located in the underground Baksan Neutrino Observatory in the Caucasus mountains of Russia, observed a neutrino rate of $67.2 \pm_{7.0}^{7.2}$ (stat.) $\pm_{3.0}^{3.5}$ (syst.) SNU, while the predicted rate for gallium is 129 SNU [32]. The GALLEX and GNO experiments, both located in the Gran Sasso Laboratory in Italy, have observed a rate of $74.1 \pm_{6.8}^{6.7} \mathrm{SNU}[33]$.

\subsubsection{Kamiokande and Super-Kamiokande}

Both the Kamiokande experiment and its successor, the Super-Kamiokande experiment have used water as a target for neutrino interactions, relying on the neutrino-electron elastic-scattering interaction,

$$
\nu+e^{-} \rightarrow \nu+e^{-}
$$

The energetic electrons, which move faster than the speed of light in water, are then detected by the cone of Cerenkov light that they produce. There are two channels by which the reaction in Equation 1.3 can proceed, one of which is possible only for electrons, while the other is possible for all flavors of neutrinos. The result is that this interaction has a cross section for electron neutrinos that is approximately 6 times larger than that for other neutrino flavors [34]. Both experiments were placed in the Kamioka mine, roughly $200 \mathrm{~km}$ west of Tokyo. The Kamiokande experiment measured a neutrino flux that was $0.492 \pm_{0.033}^{0.034}$ (stat.) \pm 0.058 (syst.) of the expected signal [35]. Based on 1496 days of data, the Super-Kamiokande collaboration has reported detecting $0.465 \pm_{.013}^{.015}$ of the expected solar neutrino signal [36] 


\begin{tabular}{|l|c|c|c|}
\hline Experiment & Observed & Expected & Reference \\
\hline \hline Homestake & $2.56 \pm 0.16$ (stat.) \pm 0.16 (syst.) SNU & $7.7_{-1.0}^{+1.3}$ SNU & {$[30,31]$} \\
\hline SAGE & $67.2 \pm_{7.0}^{7.2}$ (stat.) $\pm_{3.0}^{3.5}$ (syst.) SNU & $129_{-6}^{+8}$ SNU & {$[32]$} \\
\hline GNO and GALLEX & $74.1 \pm_{6.8}^{6.7}$ SNU & $129_{-6}^{+8}$ SNU & {$[33]$} \\
\hline Kamiokande & $0.492 \pm_{.033}^{.034}($ stat. $) \pm .058($ syst. $) \times \mathrm{SSM}$ & $1.0_{-0.14}^{+0.19}$ & {$[35]$} \\
\hline Super-K & $0.465 \pm_{.013}^{.015} \times \mathrm{SSM}$ & $1.0_{-0.14}^{+0.19}$ & {$[36]$} \\
\hline \hline
\end{tabular}

Table 1.1: Results from previous solar neutrino experiments. The uncertainties on the expected fluxes come from [31].

\subsection{The Solar Neutrino Problem}

As was described in the previous section, several experiments have made measurements of the solar neutrino flux, using a variety of targets and reactions. The results of these experiments are summarized in Table 1.1. All of the experiments have observed a significant deficit of solar neutrinos compared to the theoretical predictions. This is the heart of the solar neutrino problem. There are two possible solutions to this inconsistency: our solar model may be flawed or our understanding of the neutrino may be incomplete or incorrect.

We can examine other solar physics research to help evaluate the accuracy of our solar model. The field of helioseismology uses oscillations of the Sun's surface to probe its structure. John Bahcall writes [23], "The technique is analogous to striking a bell and using the frequencies of the emitted sound to make inferences about the bell's constitution."

Figure 1.3 shows the difference between the measured solar sound speeds (which depend on the density) and the predicted sound speeds from the 1998 Bahcall-Pinsonneault solar model. The observed discrepancies are much smaller than what would be required for the solar neutrino predictions to match the data. This makes a solution to the solar neutrino problem involving flaws in the solar model less likely. 


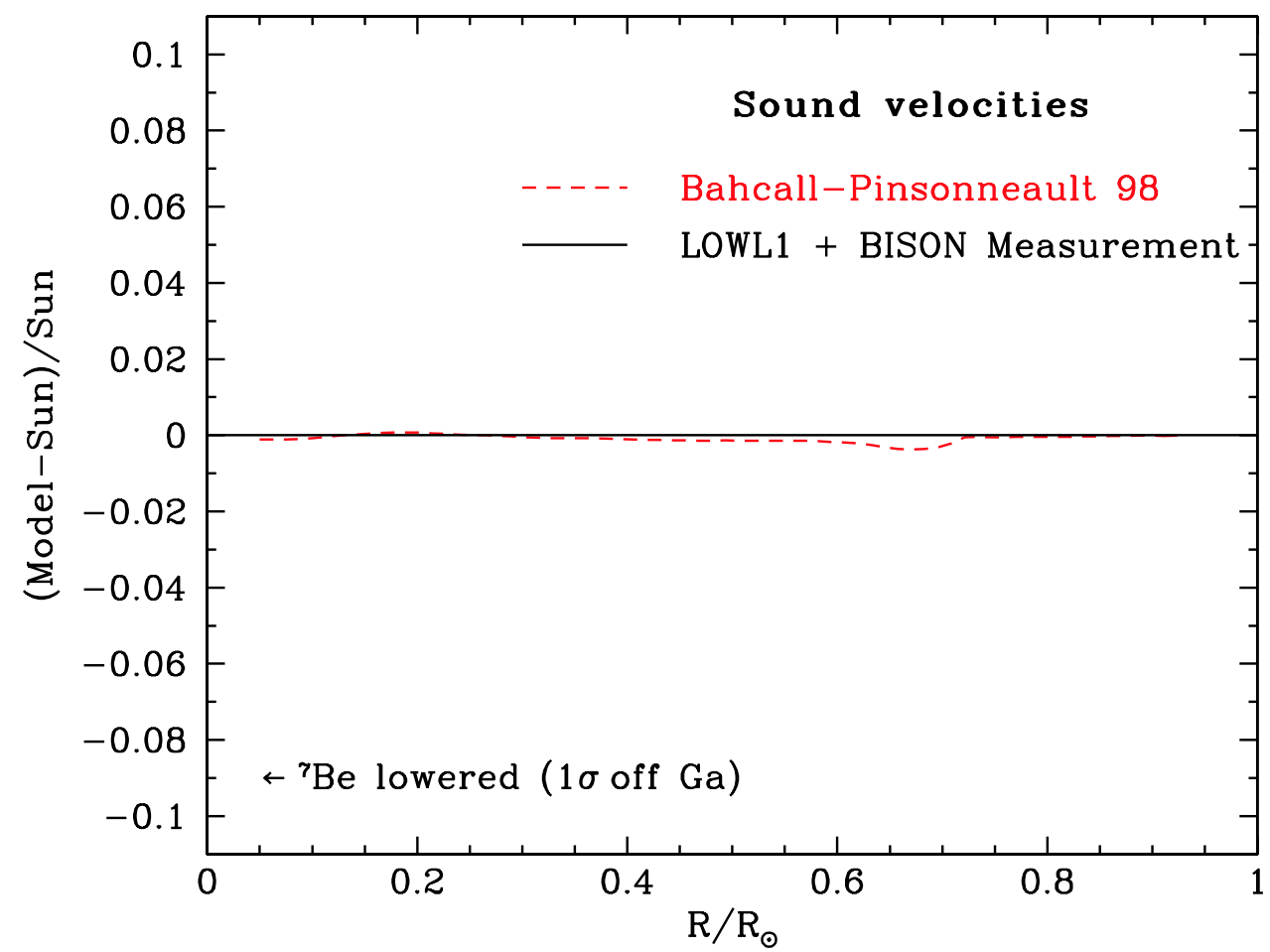

Figure 1.3: This figure, from [31], shows the difference between sound speeds from the Bahcall-Pinsonneault 1998 standard solar model and helioseismological measurements as a function of the radial position in the Sun. The arrow indicates the discrepancy which would be required to bring the solar model neutrino predictions to within $1 \sigma$ of the solar neutrino measurements. 
Another possible explanation to the solar neutrino problem involves the properties of the neutrino. As mentioned above, there are three neutrino flavors. Only one of these flavors, the electron neutrino, is produced in the core of the Sun. All of the experiments on the Earth have been primarily sensitive to electron neutrinos. If during the course of traveling from the Sun to the Earth the neutrino could somehow change flavors, then this might explain why we have detected too few electron neutrinos at the Earth. The next section will discuss how such a transformation might occur.

\subsection{Neutrino Oscillation}

We know that the there are three different neutrino flavor states $(\mathrm{e}, \mu$, and $\tau)$ that couple via the weak interaction. Now, suppose that they are composed of three mass eigenstates, which are different from the flavor eigenstates. This is really not so unreasonable, since a similar phenomenon occurs in the quark sector where the weak interaction eigenstates are different from the strong interaction eigenstates [1]. Furthermore, suppose that the flavor eigenstates, $\nu_{l}$, are a linear combination of the mass eigenstates, $\nu_{m}$, related by a matrix ${ }^{7}, U_{l m}$. We can denote the flavor eigenstates as

$$
\nu_{l}=\sum_{m=1}^{N} U_{l m} \nu_{m}
$$

where $N$ is the number of neutrino flavors. We can transform this equation to obtain an expression for the mass eigenstates,

$$
\nu_{m}=\sum_{l^{\prime}=1}^{N} U_{l^{\prime} m}^{*} \nu_{l^{\prime}} .
$$

\footnotetext{
${ }^{7}$ This section follows a treatment given in [37]
} 
Suppose at time $t=0$ that we are in a pure flavor state, $\nu_{l}$, with a momentum ${ }^{8} p$.

The wave function at time $t=0$ is

$$
\psi(x, t=0)=\sum_{m=1}^{N} U_{l m} \nu_{m} e^{i p x} .
$$

Evolving this equation in time gives

$$
\psi(x, t)=\sum_{m=1}^{N} U_{l m} \nu_{m} e^{i p x} e^{-i E_{m} t} .
$$

If the neutrino mass, $M_{m}$, is much less than the momentum $p$, then the neutrino is traveling close to the speed of light, $c$. Working in units where $c=1$ and $\hbar=1$, we have $E_{m} \simeq$ $p+M_{m}^{2} / 2 p$. Defining $x=0$ at $t=0$, we can rewrite Equation 1.7 as

$$
\psi(x, x) \simeq \sum_{m=1}^{N} U_{l m} \nu_{m} e^{-i\left[M_{m}^{2} / 2 p\right] x}
$$

Using Equation 1.5, the expression can be rewritten in terms of the $\nu_{l}$ flavor eigenstates as

$$
\psi(x, x) \simeq \sum_{l^{\prime}=1}^{N}\left[\sum_{m=1}^{N} U_{l m} e^{-i\left[M_{m}^{2} / 2 p\right] x} U_{l^{\prime} m}^{*}\right] \nu_{l^{\prime}}
$$

At time $t=0$ and $x=0$ our neutrino had flavor $l$. We can now ask what is the probability that the neutrino has flavor $l^{\prime}$ when it has reached $x$.

$$
P\left(l^{\prime}, x\right)=\left|\left\langle\psi_{l^{\prime}}(x) \mid \psi_{l}(x)\right\rangle\right|=\left[\sum_{m^{\prime}=1}^{N} U_{l m^{\prime}}^{*} e^{-i\left[M_{m^{\prime}}^{2} / 2 p\right] x} U_{l^{\prime} m^{\prime}}\right]\left[\sum_{m=1}^{N} U_{l m} e^{-i\left[M_{m}^{2} / 2 p\right] x} U_{l^{\prime} m}^{*}\right]
$$

If we now assume that $\mathrm{CP}$ is conserved, then $U$ is real, and we obtain

$$
P\left(l^{\prime}, x\right)=\left[\sum_{m=1}^{N} U_{l m}^{2} U_{l^{\prime} m}^{2}+\sum_{m \neq m^{\prime}}^{N} U_{l m} U_{l m^{\prime}} U_{l^{\prime} m^{\prime}} U_{l^{\prime} m} \cos \left(x / L_{m m^{\prime}}\right)\right],
$$

where the oscillation length, $L_{m m^{\prime}}$ is defined as

$$
L_{m m^{\prime}}=2 \pi \frac{2 p}{\left|M_{m}^{2}-M_{m^{\prime}}^{2}\right|} \equiv 2 \pi \frac{2 p}{\Delta M_{m m^{\prime}}^{2}} .
$$

\footnotetext{
${ }^{8}$ For a wave packet treatment that does not assume that the neutrinos have a perfectly defined momentum, see [38].
} 
Equation 1.11 indicates that the probability of detecting a neutrino in a given weak interaction flavor state oscillates as a function of distance. Note that for an oscillation to occur, at least some of the neutrino flavors must have non-zero masses and the masses must not be equal.

\subsubsection{Two-Flavor Oscillations}

Though it is believed that there are only three active neutrino flavors which couple to the weak interaction, typically it is assumed that one of the oscillation lengths is much longer than the other. Therefore, it is normally sufficient to think about neutrino oscillation between two flavors. In this case, the mixing matrix, $U_{l m}$, reduces to a rotation matrix,

$$
U=\left|\begin{array}{cc}
\cos (\theta) & \sin (\theta) \\
-\sin (\theta) & \cos (\theta)
\end{array}\right|,
$$

where $\theta$ is the mixing angle. The probability of starting in flavor $l$ and remaining in flavor $l$ at position $x$ is

$$
P(l \rightarrow l, x)=1-\sin ^{2}(2 \theta) \sin ^{2}(\pi x / L) .
$$

\subsubsection{Oscillations in Matter}

So far, the discussion of oscillations has only dealt with neutrinos freely traveling in a vacuum. In the presence of matter, there are additional effects which can arise. Electron neutrinos can interact with electrons via the $\mathrm{W}$ or $\mathrm{Z}$ boson, while the $\mu$ and $\tau$ neutrinos can only interact with electrons via the $\mathrm{Z}$ boson, as shown in Figure 1.4. Therefore, in the absence of free $\mu \mathrm{s}$ and $\tau \mathrm{s}$, the electron neutrino eigenstate will propagate differently than the $\mu$ and $\tau$ states. An analogous situation arises with the propagation of light in 
birefringent crystals, where the index of refraction is depends on the polarization of the light.

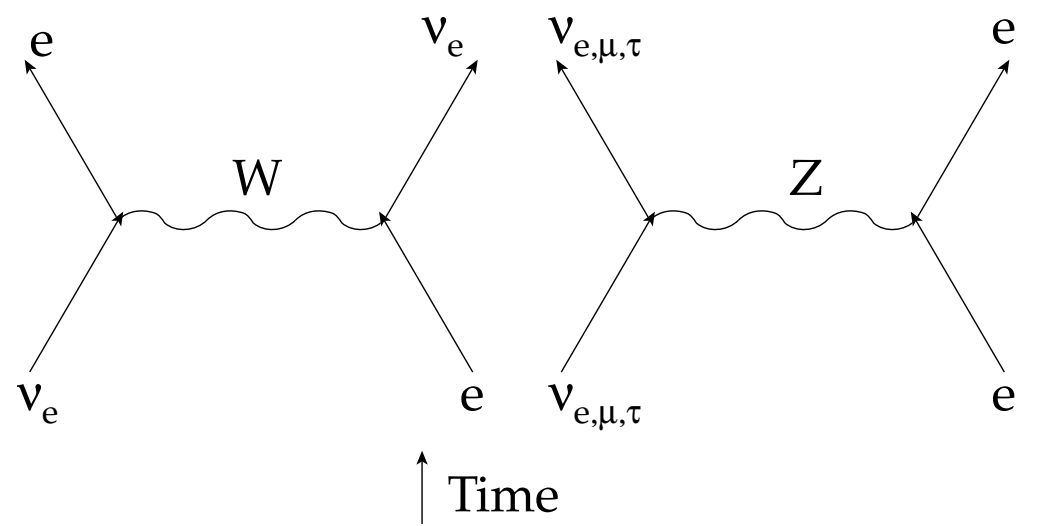

Figure 1.4: The Feynman diagrams for the possible neutrino interactions with electrons. Note that the electron neutrinos can interact via the $\mathrm{W}$ or the $\mathrm{Z}$ boson.

In the case of constant density, effects in matter will modify the result in Equation 1.14 to $[23]$

$$
P_{\text {matter }}(l \rightarrow l, x)=1-\sin ^{2}\left(2 \theta_{m}\right) \sin ^{2}\left(\pi x / L_{m}\right),
$$

where the oscillation length in matter is defined as

$$
L_{m} \equiv \frac{L}{D_{m}}=\frac{L}{\left[1-2\left(L G_{F} n_{e} / \sqrt{2} \pi\right) \cos (2 \theta)+\left(L G_{F} n_{e} / \sqrt{2} \pi\right)^{2}\right]^{1 / 2}},
$$

and the mixing angle in matter, $\theta_{m}$, is given by

$$
\sin \left(2 \theta_{m}\right)=\frac{\sin (2 \theta)}{D_{m}} .
$$

Here $G_{F}$ is the Fermi constant and $n_{e}$ is the electron number density. No new free parameters have been introduced, but instead the terms are modified by factors that depend on the electron density of the matter. Notice in these relations that there is a density where 
the $L n_{e} G_{f} / \sqrt{2} \pi=\cos (2 \theta)$, making $\sin \left(2 \theta_{m}\right)=1$, and thus a great enhancement in the mixing can occur. This enhancement is known as the MSW effect, named after Wolfenstein, who first considered the equations for neutrino propagation in matter [39], and Mikheyev and Smirnov, who appreciated the importance this could have for matter with varying density [40]. For the case of a slowly varying electron density, as is the case for neutrinos emerging from the Sun, it is possible that for some values of $\Delta M_{m m^{\prime}}^{2}$ nearly all of the electron neutrinos will emerge from the Sun as a different flavor [23]. It is interesting to note that this effect is reversible, so that if the neutrinos from the Sun enter a region of slowly increasing density, some of the neutrinos will convert back into electron neutrinos. We will actually see more electron neutrinos from the Sun at night [23].

If we combine all of the results from the solar neutrino experiments, we can determine a range of values for $\Delta M_{m m^{\prime}}^{2}$ and $\theta$ that would yield a suppression of the solar electron neutrino flux that is consistent with what is observed. Figure 1.5 shows the regions of the parameter space which were allowed prior to the results of the SNO experiment. ${ }^{9}$

\subsubsection{Other Evidence for Neutrino Oscillation}

There are now signs of neutrino oscillation from sources other than solar neutrinos. In addition to neutrinos coming from the Sun, we can also detect neutrinos which are produced in the upper atmosphere from cosmic-ray interactions. The collisions of cosmic rays with nuclei typically result in hadronic showers. The pions in the showers can decay by processes such as $\pi^{+} \rightarrow \mu^{+}+\nu_{\mu}$. The muons produced then typically decay by

\footnotetext{
${ }^{9}$ Note that $\Delta m^{2}$ can be positive or negative. In the presence of matter effects, these two scenarios will behave differently. As was pointed out in [41], one can use $\tan ^{2} \theta$, instead of $\sin ^{2} 2 \theta$, to take into account all of the possible oscillation phase space.
} 


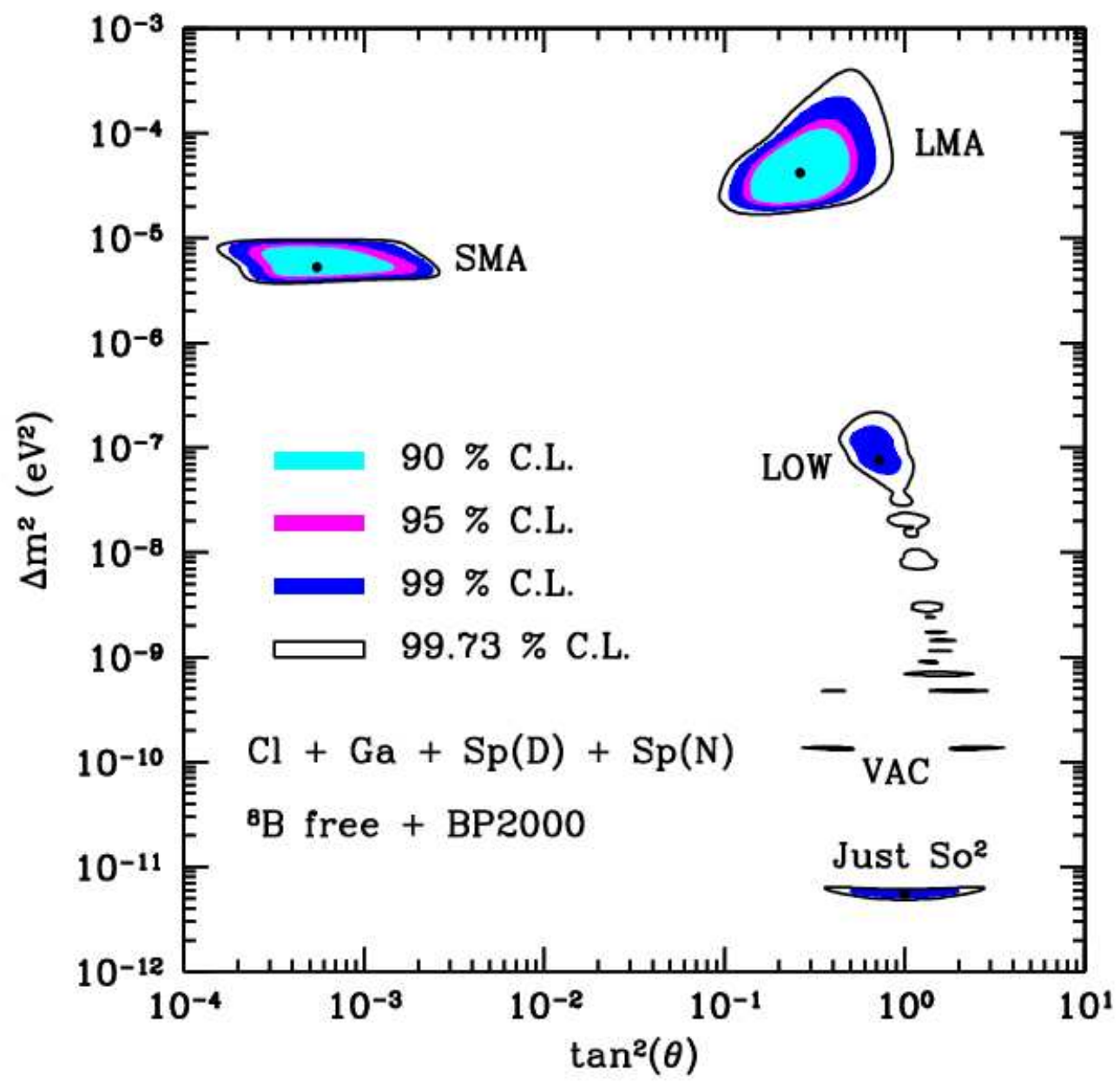

Figure 1.5: The shaded regions in this figure, from [42], show the values for $\Delta M_{m m^{\prime}}^{2}$ and $\theta$ that are consistent with the results from the solar neutrino experiments prior to the $\mathrm{SNO}$ results. Observations from the $\mathrm{Cl}, \mathrm{Ga}$, and Super-Kamiokande experiments have been included here. The regions depict the $90 \%$ confidence level region (inner light blue region), the $95 \%, 99 \%$. and the outer $99.74 \%$ confidence level region (white enclosed in black line). The name of each region is printed near it. 
$\mu^{+} \rightarrow e^{+}+\bar{\nu}_{\mu}+\nu_{e}$. The combination of these two interactions yields an expected ratio for the number of muon-type neutrinos to electron-type neutrinos of approximately two. The Super-Kamiokande experiment has performed an analysis of the atmospheric neutrino interactions occurring within their detector [43]. They see a deficit of muon neutrinos as a function of the zenith angle. Since atmospheric neutrinos reaching Super-Kamiokande from below have travelled a longer pathlength through the Earth to reach the detector than those which reach Super-Kamiokande from above, the fact that they detect fewer muon neutrinos traveling upward is consistent with the interpretation that some of the muon neutrinos have oscillated into another flavor while traveling through the Earth. Since they do not see a corresponding enhancement of upward-going electron neutrinos, this would suggest that that the $\nu_{\mu} \mathrm{s}$ have oscillated into $\nu_{\tau} \mathrm{s}$.

There is also evidence for neutrino disappearance coming from a reactor neutrino experiment. Located in western Japan, in the former home of the Kamiokande experiment, the KamLAND experiment (short for Kamioka Liquid scintillator Anti-Neutrino Detector) was designed to search for anti-neutrinos coming from commercial nuclear power reactors. ${ }^{10}$ The flux-weighted average distance between the nuclear reactors and the detector is roughly $180 \mathrm{~km}$. The observed anti-neutrino flux is $0.686 \pm 0.044$ (stat.) \pm 0.045 (syst.) of the expected signal for no oscillations [44]. Assuming CPT is invariant, the KamLAND experiment is sensitive to the same region of the oscillation parameter space as the solar neutrino experiments due to its long baseline. The deduced oscillation parameters for the KamLAND result are in good agreement with those obtained by a global analysis of the solar neutrino

\footnotetext{
${ }^{10} \mathrm{~A}$ more detailed description of the KamLAND detector and some of the work that the author did on the detector electronics can be found in Appendix A.
} 
experiments.

The Liquid Scintillator Neutrino Detector (LSND) experiment at Los Alamos also reported evidence for neutrino oscillation. The detector consisted of a large tank of liquid scintillator, surrounded by phototubes, which was placed down stream of a muon beam at the Los Alamos Meson Facility. LSND saw an excess of events in searches for both $\nu_{\mu} \rightarrow \nu_{e}$ oscillations [45] and $\bar{\nu}_{\mu} \rightarrow \bar{\nu}_{e}$ oscillations [46]. The miniBooNE experiment at Fermilab [47] has been designed to confirm the LSND signal and should have results available within the next year or two.

So far we have seen evidence for neutrino oscillation in three different regimes: solar/reactor electron neutrinos, atmospheric muon neutrinos, and accelerator muon neutrinos. Currently each of these areas requires a different value of $\Delta M_{m m^{\prime}}^{2}$. The solar results favor a $\Delta M_{m m^{\prime}}^{2}$ between $10^{-5} \mathrm{eV}^{2}$ and $10^{-4} \mathrm{eV}^{2}$, the atmospheric results favor a $\Delta M_{m m^{\prime}}^{2}$ between $2 \times 10^{-3} \mathrm{eV}^{2}$ and $3 \times 10^{-3} \mathrm{eV}^{2}$, and the LSND results favor a $\Delta M_{m m^{\prime}}^{2}$ between $4 \times 10^{-2} \mathrm{eV}^{2}$ and $1 \mathrm{eV}^{2}$ [18]. If there are only three neutrinos flavors, then the three $\Delta M_{m m^{\prime}}^{2}$ values must sum up to zero, and they do not. This has led some theorists to hypothesize the existence of a fourth (or fifth or sixth ...) neutrino species, assuming that all of the experimental results are correct. However, we already know from results at LEP [18] that there are only 3 light neutrino flavors that couple to the $\mathrm{Z}$ boson. So, these additional neutrino species must be "sterile", referring to the fact that they do not interact via the weak interaction. 


\subsection{Massive Neutrinos}

The results of numerous experiments now provide evidence for disappearing neutrinos. At the present moment the favored explanation for this disappearance is that the neutrinos are undergoing oscillations. However, as was pointed out in Equation 1.12, for these oscillations to occur, at least some of the neutrino flavors must have non-zero masses. Currently the Standard Model of particles and interactions only includes massless neutrinos and it is not such a simple matter to insert non-zero masses.

If we want to add neutrino masses into the Standard Model in the same fashion as the other lepton masses (via a so-called Dirac mass term), then this requires the existence of right-handed neutrinos and left-handed anti-neutrinos. Since these particles have not been observed in weak interactions, Dirac-type neutrinos would require some new physics to be added to the Standard Model.

On the other hand, we could instead consider adding them into the Standard Model via a Majorana-type mass term, an option only available to chargeless particles. Here the neutrino is its own anti-particle and the left-handed particles are what we call neutrinos and the right handed particles are what we call anti-neutrinos. One of the interesting things about the Majorana mass term is that it allows a particle to transform into an antiparticle [48]. This violates the conservation of lepton number, one of the principles of the Standard Model.

Therefore, if neutrino flavor oscillations can be confirmed, then there must be non-zero neutrino masses and this will require an extension of the Standard Model. The remainder of this dissertation will focus on the Sudbury Neutrino Observatory, which was 
designed with the goal of confirming solar neutrino oscillation in mind. 


\section{Chapter 2}

\section{The Sudbury Neutrino}

\section{Observatory}

\subsection{The SNO Detector}

The Sudbury Neutrino Observatory (SNO) is a large volume heavy water Čerenkov detector. The idea to construct a neutrino detector using heavy water was proposed by Herbert Chen [49], who realized that heavy water could be used to detect neutrinos of all flavors, thus providing a direct approach to resolving the solar neutrino problem.

The SNO detector is located in INCO's Creighton Mine No. 9, just outside Sudbury, Ontario, Canada. Contained in a barrel-shaped $22 \mathrm{~m}$ diameter by $34 \mathrm{~m}$ tall cavity, the detector sits beneath $2.06 \mathrm{~km}$ of rock. This depth corresponds to an overburden of $\sim 6000$ meters water equivalent $[50,51]$. An illustration of the SNO detector is shown in Figure 2.1 and an extensive description is contained in [50]. 


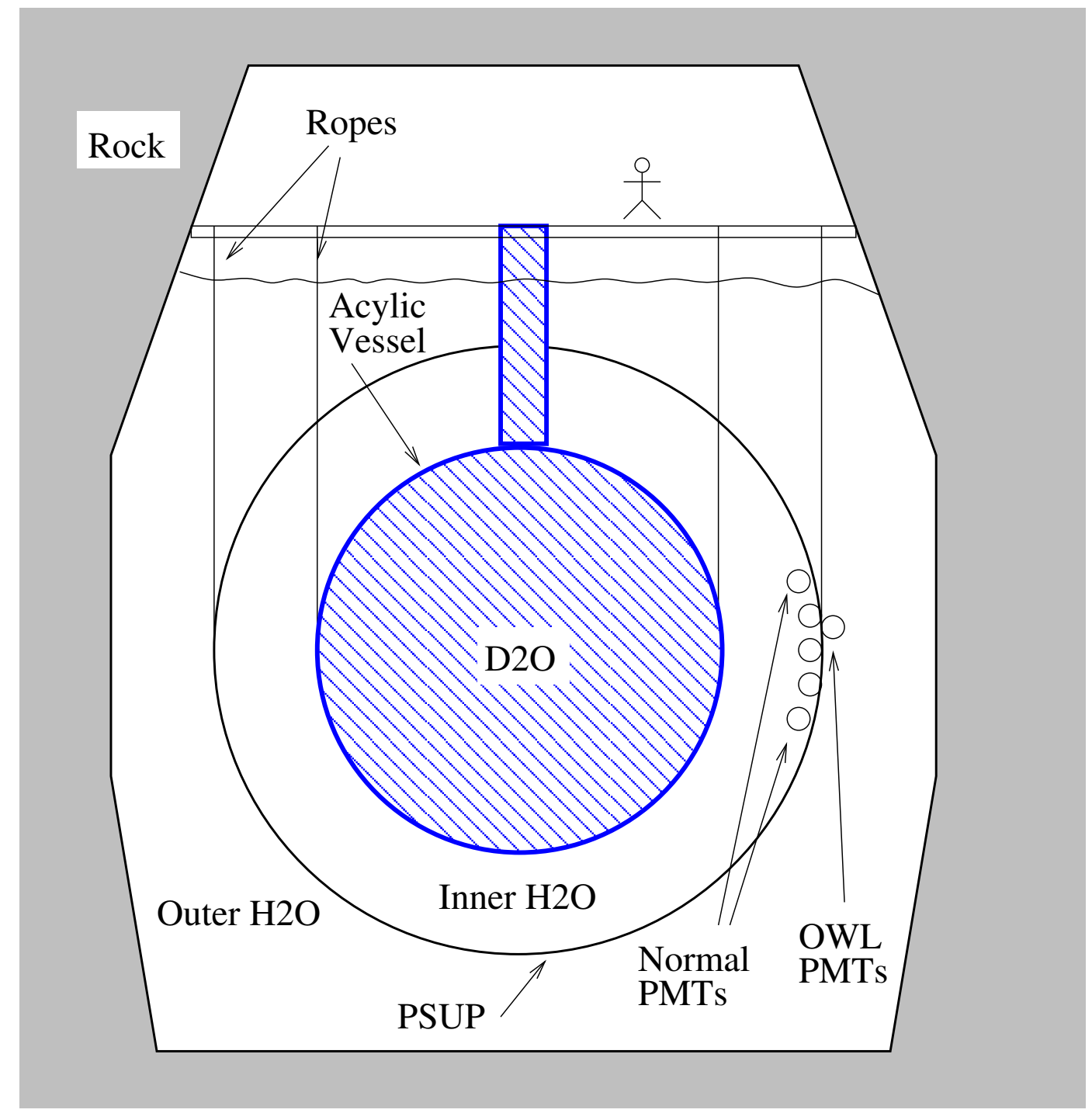

Figure 2.1: A cross section of the SNO Detector. 


\subsubsection{The Heavy Water}

At the center of this detector is 1000 metric tons of heavy water $\left(\mathrm{D}_{2} \mathrm{O}\right)$, on loan from Ontario Hydro. The composition of the hydrogen in the heavy water is $99.917 \%{ }^{2} \mathrm{H}$ (with the balance as ${ }^{1} \mathrm{H}$ ), while the oxygen content is $99.12 \%{ }_{8}^{16} \mathrm{O}, 0.17 \%{ }_{8}^{17} \mathrm{O}$, and $0.71 \%$ ${ }_{8}^{18} \mathrm{O}$ [50]. In May 2001, 2 metric tons of $\mathrm{NaCl}$ were mixed into the $\mathrm{D}_{2} \mathrm{O}$, resulting in a $0.2 \%$ brine solution. The salt was removed from the water in September 2003, primarily by the use of a reverse-osmosis unit. Spectroscopic analysis indicated that the level of $\mathrm{NaCl}$ remaining in the $\mathrm{D}_{2} \mathrm{O}$ following the salt removal is $<1 \mathrm{ppm}[52]$.

\subsubsection{The Acrylic Vessel}

The $\mathrm{D}_{2} \mathrm{O}$ is contained by an acrylic vessel $(\mathrm{AV})$. Consisting of a $12.12 \mathrm{~m}$ diameter sphere with a $1.5 \mathrm{~m}$ diameter, $6.8 \mathrm{~m}$ tall cylindrical chimney [50], the AV is shown in Figure 2.2. The chimney allows access to the $\mathrm{D}_{2} \mathrm{O}$ for the insertion of calibration sources into the $\mathrm{D}_{2} \mathrm{O}$ and for pipes to circulate the $\mathrm{D}_{2} \mathrm{O}$. The $\mathrm{AV}$ is supported by 10 loops of a specially designed rope of Vectran filaments.

The sphere of the AV is composed of 122 panels of ultra-violet transmitting (UVT) acrylic. UVT acrylic was chosen to allow greater transmission of Čerenkov light from the $\mathrm{D}_{2} \mathrm{O}$ in the region of peak PMT sensitivity. The panels were bonded together using a partially polymerized adhesive. They are $5.6 \mathrm{~cm}$ thick, except for the equatorial panels housing the suspension ropes, which are $10 \mathrm{~cm}$ thick. The chimney was fabricated from ultraviolet-absorbing (UVA) acrylic to reduce the light entering the detector from regions other than the central $\mathrm{D}_{2} \mathrm{O}$ sphere. 


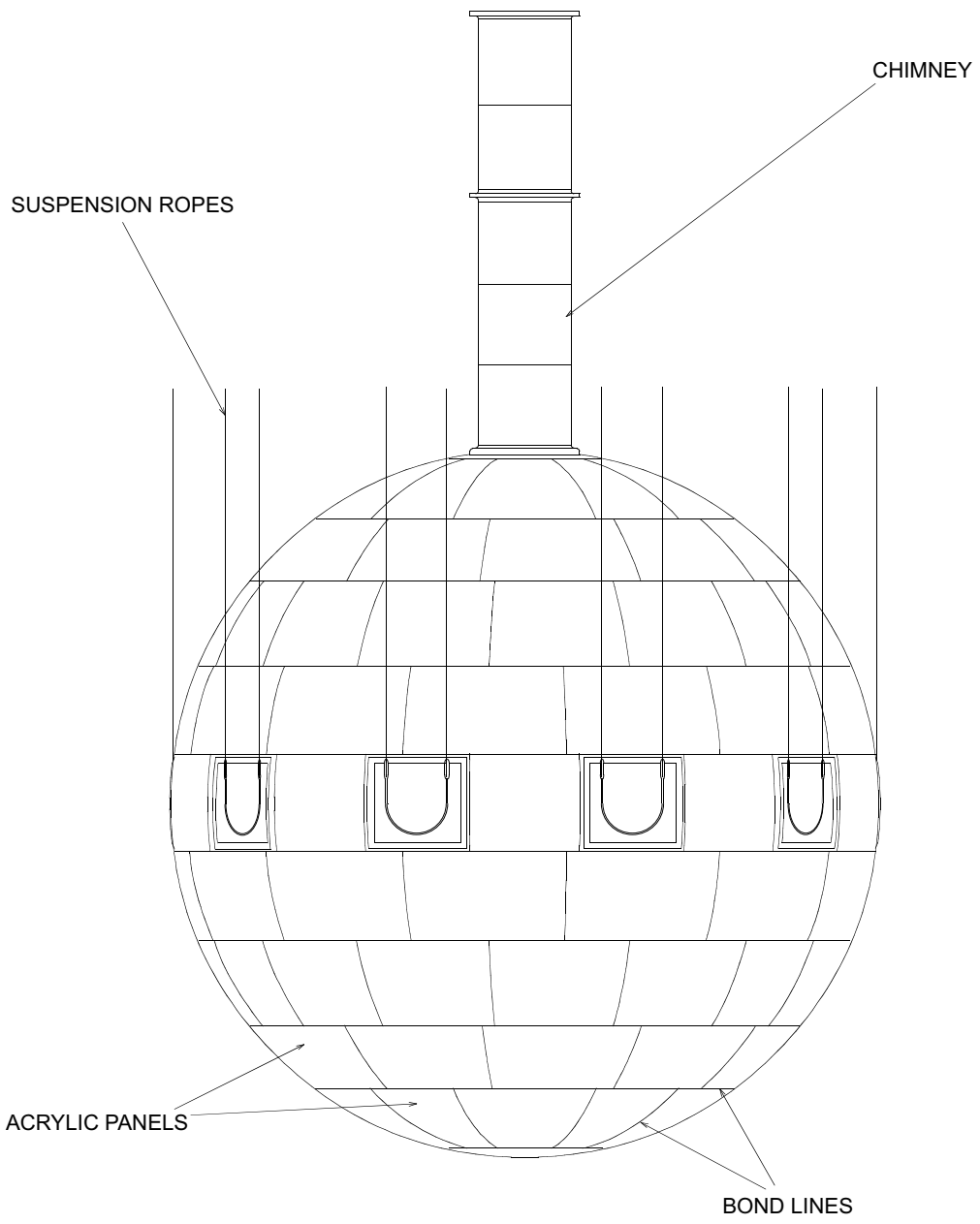

Figure 2.2: The SNO acrylic vessel which contains the $\mathrm{D}_{2} \mathrm{O}$. 


\subsubsection{The Light Water}

Surrounding the AV is 7400 metric tons of ultrapure $\mathrm{H}_{2} \mathrm{O}$, with 1700 metric tons in the Inner $\mathrm{H}_{2} \mathrm{O}$ region between the PMTs and the $\mathrm{AV}$, and 5700 metric tons in the Outer $\mathrm{H}_{2} \mathrm{O}$ region filling the remainder of the cavity. The water provides buoyancy to help support the weight of the $\mathrm{D}_{2} \mathrm{O}$ and the PMTs and also provides shielding from neutrons and high-energy gammas coming from the rock walls.

\subsubsection{The Photo-multiplier Tubes and PMT Support Structure}

Submerged into the $\mathrm{H}_{2} \mathrm{O}$ is the PMT Support Structure (PSUP). The stainless steel PSUP is a $889 \mathrm{~cm}$ radius geodesic composed of triangles joined at 91 nodes [50], as depicted in Figure 2.3. The primary purpose of this structure is to support the photomultiplier tubes (PMTs) which detect the light produced by neutrino interactions. The structure also needed to be lightweight, low in radioactivity, and water-tight. There are 9438 PMTs which look inward at the $\mathrm{D}_{2} \mathrm{O}$ volume. Each inward-looking PMT is housed in a hexagon constructed of black acrylonitrile-butadiene-styrene (or ABS) plastic. The hexagons support light concentrators which are attached to the faces of the PMTs. The concentrators consist of 18 thin curved "petals" of aluminum which have been coated in a dielectric to reduce corrosion. Detailed in [53], the concentrators increase the effective area of each PMT by $75 \%$ and have been tuned so that the PMTs are most sensitive to light coming from within the $\mathrm{D}_{2} \mathrm{O}$. The hexagonal PMT housings are bolted together into panels which are affixed to the PSUP frame. Since the plastic of the hexagons is opaque, it provides a light barrier between the Inner and Outer $\mathrm{H}_{2} \mathrm{O}$. There are an additional 91 outward-looking PMTs (or OWLs) 
which look for light produced in the Outer $\mathrm{H}_{2} \mathrm{O}$ (Figure 2.1) to serve as a tag for cosmic muons that originate outside of the detector. There are also four "neck" PMTs mounted at the top of the AV chimney to tag static discharges occurring on the AV chimney near the air/water interface.

SNO uses $20.4 \mathrm{~cm}$ diameter Hamamatsu R1408 PMTs. The envelopes surrounding the PMTs are composed of a special glass (Schott 8246), which has low intrinsic Th and U levels $(<100 \mu \mathrm{g}$ per PMT [50]). The 9438 inward-facing PMTs provide a photocathode coverage of $31 \%$. The addition of the light concentrators increases the effective coverage to $54 \%$ [50]. The photo-multiplier tube efficiency, detailed in [54], peaks at a value of $\sim 13 \%$ in the range of 350 to $475 \mathrm{~nm}$ (blue to ultraviolet). The efficiency falls under $1 \%$ below 250 $\mathrm{nm}$ and above $625 \mathrm{~nm}$. The Earth's magnetic field can reduce the PMT efficiency by up to $20 \%$ [55]. Due to its northern location, the magnetic field at SNO only deviates from vertical by $15^{\circ}$. The vertical component of the Earth's magnetic field is cancelled by 14 field compensation coils running through the cavity walls. The residual horizontal component which is not cancelled reduces the efficiency by less than $3 \%$.

\subsubsection{The Coordinate System}

Since the detector is largely spherical, it is convenient to define $x=0, y=0, z=0$ to be at the center of the $\mathrm{D}_{2} \mathrm{O}$ region. The positive z-axis is defined to be pointing upward through the AV chimney (or toward the surface), the positive x-axis is aligned $40.4^{\circ}$ east of true north (with respect to the Earth's rotation axis), and the positive y-axis is aligned $49.6^{\circ}$ west of true north [56]. 


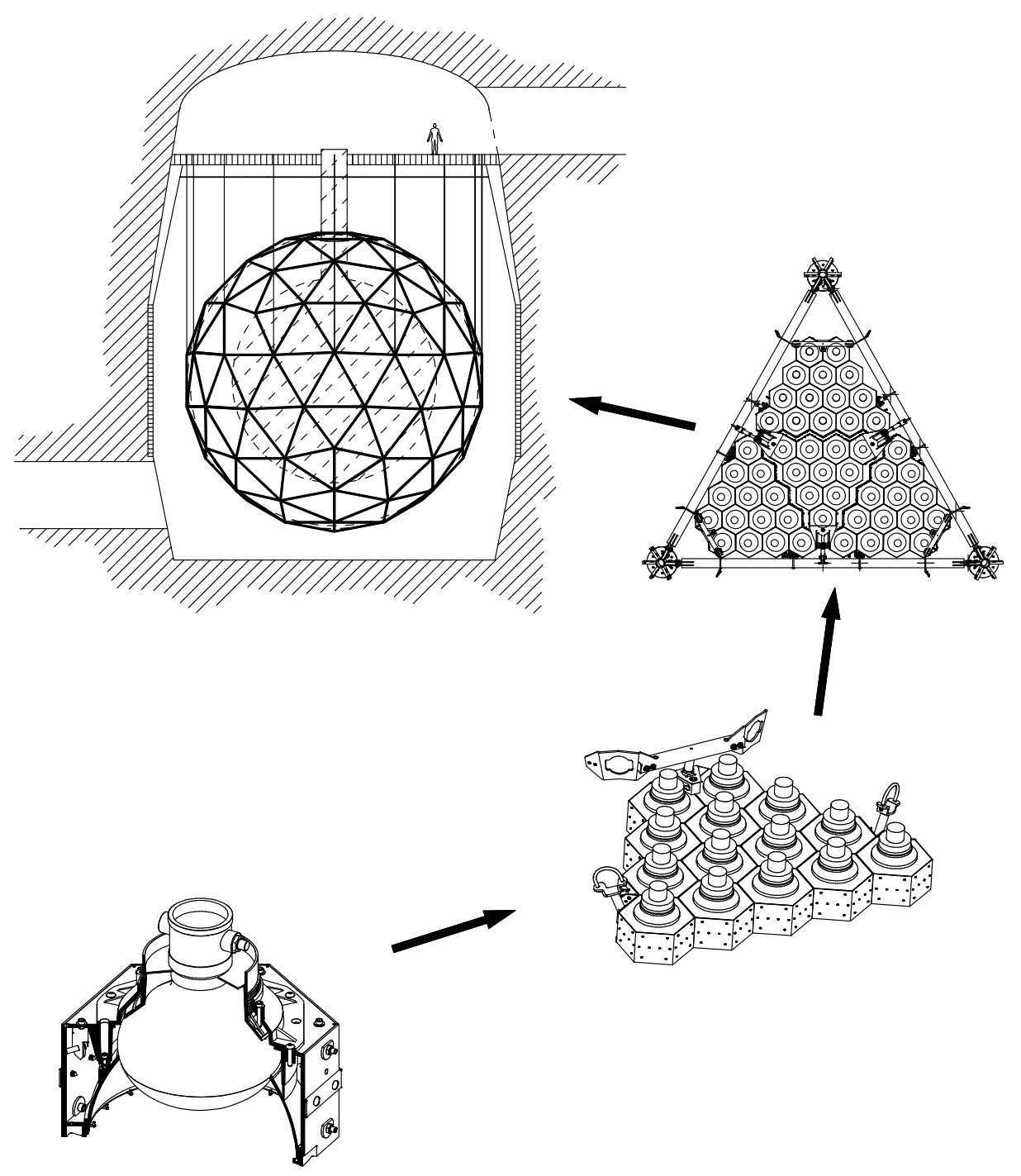

Figure 2.3: The PMT Support Structure (PSUP), showing how the hexagonal PMT housings are arranged together into panels and placed in the detector. 


\subsubsection{Electronics and Data Acquisition}

As SNO is a real time solar neutrino detector, it is important to have fast electronics, with as little dead time as possible. Though the detector typically has a trigger rate of $\sim 20 \mathrm{~Hz}$, the electronics were designed to handle sustained rates of up to $1 \mathrm{kHz}$ (necessary during calibrations, etc.) and bursts of up to $1 \mathrm{MHz}$. The design of the electronics system is more fully described in $[50,57]$. A brief summary of the system will be given here.

The electronics for the PMTs is distributed between 19 crates. Each crate houses 16 cards, with each card serving 32 PMT channels. The PMTs are attached to the cards by a single $75 \Omega$ cable which transmits both the high voltage and the PMT output signal. The pulse from a PMT is first fed into a discriminator. If the pulse is above the discriminator threshold, then a 400 ns timing cycle will begin. The PMT pulse is then fed through a low-gain and a high-gain integrator to determine the total charge on the PMT. If a global trigger signal is received during the $400 \mathrm{~ns}$ cycle, then the timing and charge information from that PMT is recorded. If no global trigger is received within $400 \mathrm{~ns}$, the PMT channel resets itself.

Several different conditions can produce a global trigger. Typically we want to trigger the detector when a flash of Čerenkov light is produced. This can result in a large number of photo-multiplier tube hits or a large amount of deposited charge. Since it takes light roughly 66 ns to travel across the detector, a typical global trigger condition requires 18 photo-multiplier tubes $(\sim 3 \mathrm{MeV})$ to register a signal above the discriminator threshold within $100 \mathrm{~ns}$ of each other. To record muon events, the detector will also trigger if $12 \mathrm{OWL}$ tubes register a signal. In additional to the triggers designed to record physics events, there 
are also a number of diagnostic triggers, such as a pulsed global trigger, which forces a readout of the detector 5 times a second, and an asynchronous forced trigger, which forces a global trigger after every 1000 triggers. Both of these triggers are useful for identifying the background photo-multiplier tube rates. There is also a trigger that records events when a signal is detected on a hydrophone underground. Such a signal might be caused by blasting

or some other seismic event. While an individual channel has a deadtime of $400 \mathrm{~ns}$, the overall trigger deadtime is less than $10 \mathrm{~ns}$. As the typical trigger rate in the detector is on the order of $20 \mathrm{~Hz}$, the trigger deadtime is negligible.

It is also necessary to have accurate absolute time information, especially in the event of a supernova. SNO uses two clocks to determine the absolute event time [50]. A GPS system provides a $10 \mathrm{MHz}$ clock signal, accurate to within $100 \mathrm{~ns}$, to provide the Universal time for synchronization with other detectors. The GPS system is located on the surface, and is connected to the electronics via a $4 \mathrm{~km}$ fiber optic cable, on which the transmission time is continuously monitored. A $50 \mathrm{MHz}$ quartz oscillator located underground provides more accurate timing between events.

\subsubsection{Calibration System and Calibration sources}

SNO uses a variety of calibration sources to understand the optical, neutron capture, and energy response of the detector. SNO has two methods for deploying sources in the $\mathrm{D}_{2} \mathrm{O}$ [50]. The first is a vertical cable that can be used to position sources inside the $\mathrm{D}_{2} \mathrm{O}$ along the $\mathrm{z}$-axis. SNO also has a calibration deployment system which can move sources along a plane in the $\mathrm{D}_{2} \mathrm{O}$ by means of two vectran ropes which are attached to fixed points near the equator of the AV, as shown in Figure 2.4. SNO has two sets of such ropes, 
one that can move a source in the $\mathrm{x}-\mathrm{z}$ plane and one that can move a source in the $\mathrm{y}-\mathrm{z}$ plane. There are also a series of tubes which penetrate the PSUP to allow sources to be lowered in the $\mathrm{H}_{2} \mathrm{O}$.

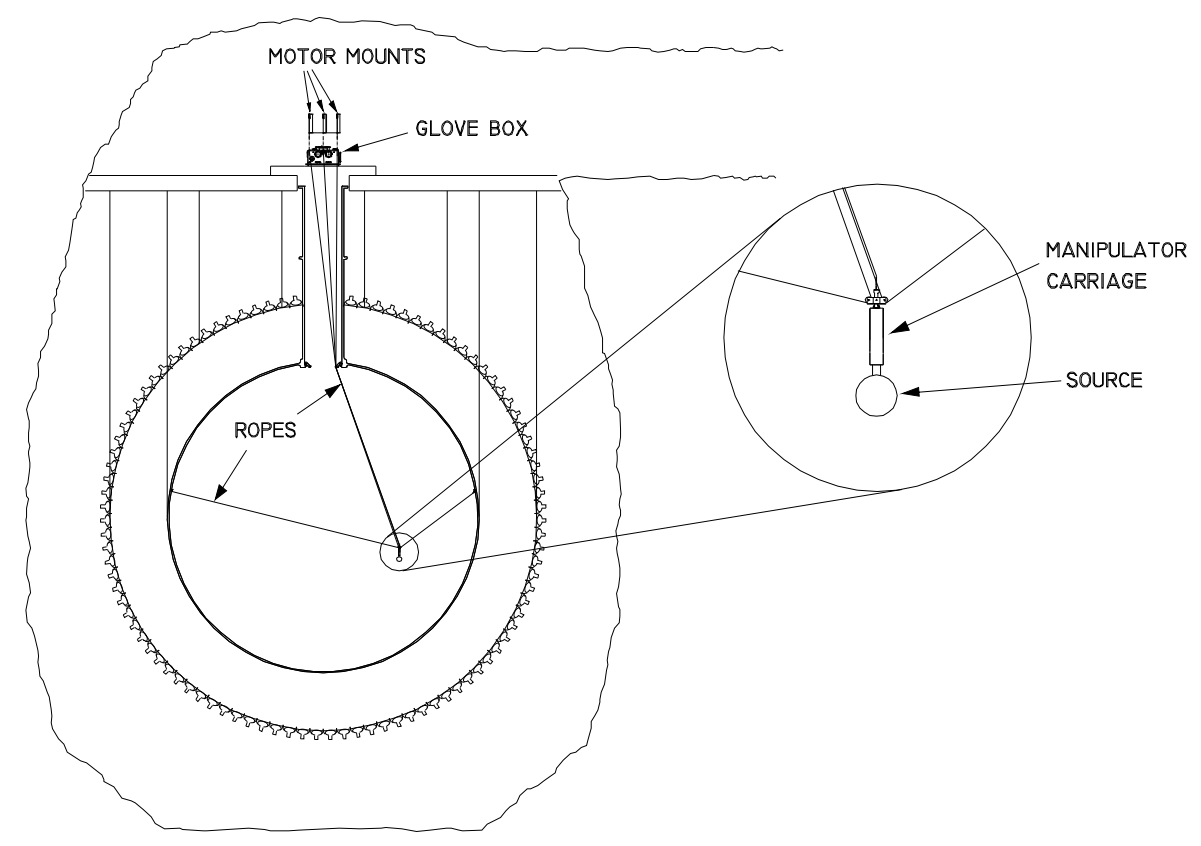

Figure 2.4: The calibration source manipulator system that can move the source along a plane of the detector.

\section{The Laserball}

For the optical and some of the electronics timing calibrations a laserball source is used [58]. A laser on the deck produces light, which is transmitted via a fiber-optic cable to a diffuser ball that can be deployed into the detector. A series of dyes can be used to can be used to shift the output of the laser to different wavelengths from 337-620 nm, with 600 ps pulses [50]. Neutral density filters adjust the intensity of the light. 


\section{The ${ }^{16} \mathbf{N}$ Source}

The primary calibration source used in the evaluation of the energy response of the detector is a ${ }^{16} \mathrm{~N}$ source [59]. A deuterium-tritium generator, located underground near the detector, produces neutrons, which yield ${ }^{16} \mathrm{~N}$ via an $(\mathrm{n}, \mathrm{p})$ interaction on the ${ }^{16} \mathrm{O}$ in $\mathrm{CO}_{2}$ gas. The gas travels into the ${ }^{16} \mathrm{~N}$ source chamber via a capillary tube. The ${ }^{16} \mathrm{~N}$ source consists of a hollow chamber surrounded by plastic scintillator, inside of a stainless steel cylinder. With a $7.13 \mathrm{sec}$ half-life, ${ }^{16} \mathrm{~N}$ emits a $6.13 \mathrm{MeV} \gamma$ along with a $\beta$ in $66.2 \%$ of its decays [60]. A PMT is used to detect the light from a $\beta$ in the scintillator, providing a tag for the emission of a $6.13 \mathrm{MeV} \gamma$-ray. ${ }^{1}$

\section{The ${ }^{252}$ Cf Neutron Source}

To probe the neutron response, $\mathrm{SNO}$ uses a ${ }^{252} \mathrm{Cf}$ fission source. The source consists of ${ }^{252} \mathrm{Cf}$ encased in acrylic and enclosed in a stainless steel sheath. The fission decay of ${ }^{252} \mathrm{Cf}$ yields an average of 3.773 neutrons per fission [61].

\section{The AmBe n-Source}

SNO also has an additional neutron source, a ${ }^{214} \mathrm{Am}-{ }^{9} \mathrm{Be}$ source. In this source, ${ }^{241} \mathrm{Am}$ emits $\alpha$-rays which strike a ${ }^{9} \mathrm{Be}$ target, producing neutrons via the ${ }^{9} \operatorname{Be}(\alpha, n){ }^{12} \mathrm{C}$ reaction [62]. For the energy spectrum of $\alpha$ s coming from ${ }^{241} \mathrm{Am}, 59.1 \pm 1.5$ percent of the reactions [63] yield a 4.439 $\mathrm{MeV}$ accompanying the neutron. In the remainder of the decays, a neutron is emitted and ${ }^{12} \mathrm{C}$ is in the ground state. Therefore, this source can potentially

\footnotetext{
${ }^{1}$ Actually in $4.8 \%$ of the decays a $7.11 \mathrm{MeV}$ is emitted in coincidence with the $\beta$. This higher energy $\gamma$-ray is fully modeled in the Monte Carlo simulation.
} 
be employed as a tagged-neutron source or as a source of $4.4 \mathrm{MeV} \gamma$-rays.

\section{The ${ }^{8}$ Li $\beta$ Source}

A tagged $\beta$ source for SNO utilizes the decay of ${ }^{8} \mathrm{Li}$. Arising from a deuteriumtritium accelerator, neutrons strike a ${ }^{11} \mathrm{~B}$ target, producing ${ }^{8} \mathrm{Li}$ via the ${ }^{11} \mathrm{~B}(n, \alpha){ }^{8} \mathrm{Li}$ interaction. The radioactive ${ }^{8} \mathrm{Li}$ gas is then transported via tubing into the source container deployed in the detector [64]. ${ }^{8} \mathrm{Li}$ typically decays by the emission of $2 \alpha$ s and a $\beta^{-}$with a maximum energy of $16 \mathrm{MeV}$ [60]. By looking for the scintillation light produced by the $\alpha \mathrm{s}$ in ${ }^{4}$ He gas in the source chamber, the $\beta$ emission is tagged.

\section{The pT Source}

To calibrate the high-energy response of the detector, a pT source was developed $[65,66]$. Inside of the source, protons are accelerated and strike a tritium $\left({ }^{3} \mathrm{H}\right)$ target, producing ${ }^{4} \mathrm{He}$ by a $(\mathrm{p}, \gamma)$ reaction. Since ${ }^{4} \mathrm{He}$ does not have a bound excited state, a monoenergetic $19.8 \mathrm{MeV}$ is emitted.

\subsection{Monte Carlo Simulation Package}

The multipurpose SNO Monte Carlo simulation and analysis software -known as SNOMAN- has been developed over the past decade with significant contributions from many authors [67].

The SNOMAN package is primarily written in FORTRAN and relies upon the CERN Program Library, including the ZEBRA [68] memory manager and the GEANT Version 3 [69] detector description and simulation tool. To simulate the interactions of 
the electron, photons, and other particles, SNOMAN uses several packages including EGS4 (to simulate electron and $\gamma$-ray interactions, [70]), FLUKA (high-energy hadronic interactions, [71]), MCNP (from Los Alamos for the simulation of neutron interactions, [72]), MUSIC (MUon SImulation Code, see [73]), the Soudan 2 astronomy library, and MSW code developed by Naoya Hata [74]. SNOMAN also includes various routines to assist in the processing of the real neutrino data such as event vertex reconstruction and event filtering to remove event backgrounds. SNOMAN writes out analyzable files in both the HBOOK format [75] (for use with PAW [76]) or ROOT format [77].

\subsection{Neutrino Interactions in SNO}

Inside of the heavy water target of the SNO detector, there are three types of neutrino interactions which can occur [34]:

$$
\begin{gathered}
\nu_{x}+e^{-} \rightarrow \nu_{x}+e^{-} \\
\nu_{e}+d \rightarrow p+p+e^{-} \\
\nu_{x}+d \rightarrow \nu_{x}+p+n
\end{gathered}
$$

The first of these reactions, given by Equation 2.1, is an elastic-scattering (ES) interaction. It should be noted that this interaction can occur for any neutrino flavor, $\nu_{x}$, but the cross section is 6 to 7 times greater for $\nu_{e}[23] .^{2}$ The scattered electron direction is highly correlated with the incident neutrino direction. For example, above an electron

\footnotetext{
${ }^{2}$ The exact ratio depends on the energy threshold. For a kinetic energy above $5.0 \mathrm{MeV}$, the value is $6.64[23]$.
} 
kinetic energy threshold of $5.0 \mathrm{MeV}, 90 \%$ of the electrons will be scattered within $15^{\circ}$ of the incident neutrino direction [23, pg. 234]. Since it involves only electrons, this interaction occurs in both light water and heavy water.

The charged-current $(\mathrm{CC})$ reaction, Equation 2.2, is unique since it can occur for only $\nu_{e}$. The electron energies are well correlated with the neutrino energy and are typically 1.44 MeV below the neutrino energies [34]. The angular distribution of the electrons with respect to the neutrino direction in the center-of-mass frame can be described by $1-\frac{1}{3} \cos \theta_{\nu}$, meaning that there are actually slightly more electrons which are emitted backward than forward.

The final reaction, the neutral-current (NC) reaction of Equation 2.3, involves the breakup of a deuteron by a neutrino and has a threshold of $2.2 \mathrm{MeV}$. This is an extremely important reaction to study because it is equally sensitive to all neutrino flavors and can measure the total solar neutrino flux. If the neutrino flux observed via the $\mathrm{NC}$ interaction is significantly larger than the flux observed via the CC or ES interactions, then this is a smoking gun for neutrino flavor transformations.

While the ES and CC interactions produce an electron as the final product, the NC interaction yields a neutron. Relativistic electrons in the detector produce a cone of Cerenkov light, which provides both electron energy and directional information. On the other hand, the neutrons that are produced in the detector will rapidly thermalize and walk randomly around the detector before they eventually capture on a nucleus and produce an observable signal. As a result of this process, any information about the initial neutrino energy and direction is lost for the neutral-current reaction. 
Since the NC interaction is very important and is not observed by other experiments, the SNO detector has implemented three different methods for the detection of neutrons over the course of data taking. The first phase consisted of pure $\mathrm{D}_{2} \mathrm{O}$ inside the AV. A free neutron randomly walks around the $\mathrm{D}_{2} \mathrm{O}$ before capturing on a nucleus. If the neutron captures on ${ }^{2} \mathrm{H}$ in the $\mathrm{D}_{2} \mathrm{O}$ then a $6.25 \mathrm{MeV} \gamma$-ray is produced [50]. On the other hand, if the neutron reaches the acrylic vessel, there is a high probability that it will capture on ${ }^{1} \mathrm{H}$, due to the larger $(\mathrm{n}, \gamma)$ cross section, yielding only a $2.2 \mathrm{MeV} \gamma$-ray, which is below the analysis threshold. The neutrons that reach the AV are essentially lost.

In May 2001, the second phase of neutron detection began with the injection of 2 tonnes of $\mathrm{NaCl}$ into the heavy water. The benefit of adding ${ }^{35} \mathrm{Cl}$ is two-fold. Since ${ }^{35} \mathrm{Cl}$ has a $(n, \gamma)$ cross section which is over 80,000 times larger than that of ${ }^{2} \mathrm{H}$, more neutrons will capture within the $\mathrm{D}_{2} \mathrm{O}$ region [60]. Also, a neutron capture on ${ }^{35} \mathrm{Cl}$ results in a cascade of $\gamma$-rays with a total energy of $8.6 \mathrm{MeV}$, allowing more of the neutron interactions to be observed above the detector energy threshold.

In September 2003 the salt was removed from the $\mathrm{D}_{2} \mathrm{O}$ via a reverse-osmosis processor. The third phase of neutron detection, using an array of ${ }^{3} \mathrm{He}$ proportional counters in the $\mathrm{D}_{2} \mathrm{O}$, began in December 2003. The ${ }^{3} \mathrm{He}$ counters, referred to as Neutral-Current Detectors (or NCDs), consist of strings of $5.08 \mathrm{~cm}$ diameter nickel tubes which are filled with ${ }^{3} \mathrm{He}-\mathrm{CF}_{4}$ gas and are anchored to the bottom of the acrylic vessel. They detect neutrons by the reaction

$$
{ }^{3} \mathrm{He}+n \rightarrow p+{ }^{3} \mathrm{He}+764 \mathrm{keV} .
$$

The proton-triton pair then ionizes the gas in the counter, which is detected on the high- 
voltage wire that runs through the center of the NCD tube. To help distinguish between neutron interactions and $\alpha$ and $\beta$ backgrounds (primarily from the NCD walls), the output signals are digitized so that pulse-shape discrimination can be used. The NCDs have very different systematics than the Čerenkov light analyses and therefore provide an important confirmation of the NC results. A side view of the detector with the NCDs in place is shown in Figure 2.5.

\subsection{Previous Solar Neutrino Results from SNO}

This thesis will focus on the second phase of the SNO detector, the $\mathrm{D}_{2} \mathrm{O}+\mathrm{NaCl}$ phase. However, important results have already been published using the pure $\mathrm{D}_{2} \mathrm{O}$ data. The first results from SNO [78] gave a measurement of the neutrino flux observed via the CC interaction of

$$
\left[1.75 \pm 0.07 \text { (stat) } \pm_{0.11}^{0.12}(\text { syst }) \pm 0.05(\text { theor })\right] \times 10^{6} \mathrm{~cm}^{-2} \mathrm{~s}^{-1}
$$

This is $3.3 \sigma$ below the solar neutrino flux measured by the Super-Kamiokande experiment using the ES reaction [78]. Since the ES reaction has a component that involves non-electron neutrinos, the discrepancy between these two results hints that many of the neutrinos from the Sun have changed to other flavors.

The second publication of the SNO collaboration provides even more convincing evidence for oscillations by providing measurements of the solar neutrino flux observed via the CC, ES and $\mathrm{NC}$ interactions during the pure $\mathrm{D}_{2} \mathrm{O}$ phase. The results yielded a solar neutrino flux observed via the NC interaction of

$$
\left[5.09 \pm_{0.43}^{0.44}(\text { stat }) \pm_{0.43}^{0.46} \text { (syst) }\right] \times 10^{6} \mathrm{~cm}^{-2} \mathrm{~s}^{-1}
$$




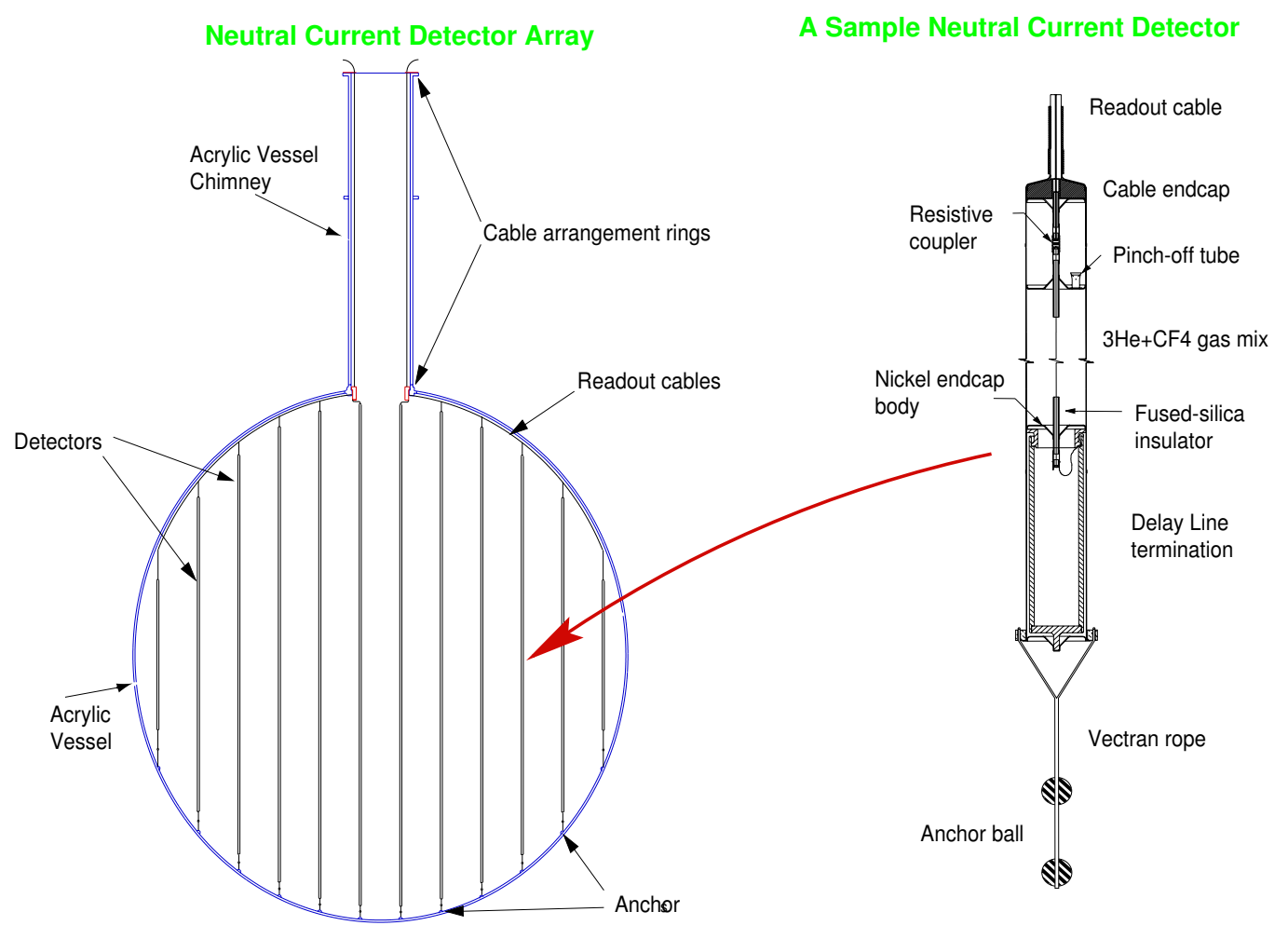

Figure 2.5: A view of the SNO acrylic vessel with the NCDs. 
for neutrinos with a kinetic energy above 5.0 MeV [79], which is in excellent agreement with the Standard Solar Model predicted flux of $\left[5.05 \pm_{0.81}^{1.01}\right] \times 10^{6} \mathrm{~cm}^{-2} \mathrm{~s}^{-1}[80]$. This provided the first direct measurement of the total active flux of neutrinos coming from the ${ }^{8} \mathrm{~B}$ reaction in the Sun. Since the flux of electron neutrinos observed [79] via the CC interaction was only,

$$
[1.76 \pm 0.05(\text { stat }) \pm 0.09(\text { syst })] \times 10^{6} \mathrm{~cm}^{-2} \mathrm{~s}^{-1}
$$

the non-electron neutrino component of the solar neutrino flux can be deduced to be

$$
\left[3.41 \pm_{0.45}^{0.45} \text { (stat) } \pm_{0.45}^{0.48} \text { (syst) }\right] \times 10^{6} \mathrm{~cm}^{-2} \mathrm{~s}^{-1}
$$

which is $5.3 \sigma$ above zero. Therefore these results strongly suggest that the electron neutrinos generated in the core of the Sun are transforming into other flavors. 


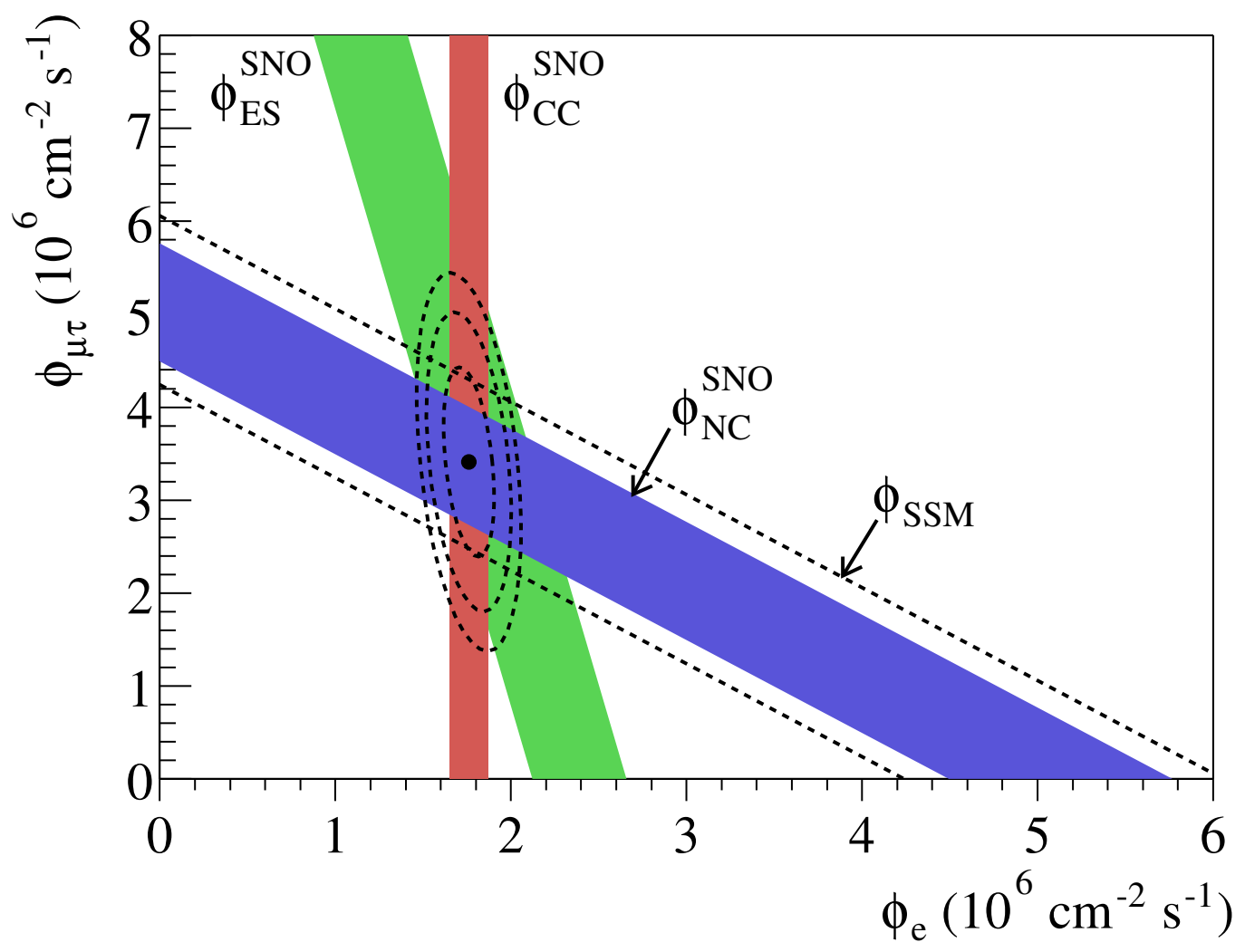

Figure 2.6: The solid bands show the sum of the $\mu$ and $\tau$ neutrino fluxes vs the electron neutrino flux for the CC, ES and $\mathrm{NC}$ reaction in $\mathrm{SNO}$ in the $\mathrm{D}_{2} \mathrm{O}$ phase (with $1 \sigma$ errors). The diagonal dashed lines show the prediction of the standard solar model [80]. This figure is taken from [79]. 


\section{Chapter 3}

\section{Event Reconstruction}

\subsection{Event Reconstruction Algorithm}

In order to analyze the neutrino signals in the data, it is important to understand where a given event originated from in the detector. Spatial reconstruction is important because this information can assist in distinguishing the various neutrino signals, as the ES interaction occurs in both the $\mathrm{H}_{2} \mathrm{O}$ and $\mathrm{D}_{2} \mathrm{O}$ and the $\mathrm{CC}$ and $\mathrm{NC}$ interactions are restricted to the $\mathrm{D}_{2} \mathrm{O}$. Good spatial resolution is also important because we would like to minimize the number of low-energy background events $(\beta-\gamma \mathrm{s}$ from $\mathrm{U}$ and Th chain activity, described in Chapter 8) from the $\mathrm{H}_{2} \mathrm{O}$, acrylic vessel, and PMTs that misreconstruct within the fiducial volume. To keep the backgrounds at an acceptable level, the fiducial volume is the inner $550 \mathrm{~cm}$-radius sphere of the AV. Again, the center of the coordinate system is defined to be the center of the $\mathrm{D}_{2} \mathrm{O}$ sphere, with the positive z-axis going through the AV chimney.

We also need to understand the event direction. This is particularly important

for the ES signal where the electron direction is highly correlated with the direction of the 
incident neutrino.

The reconstruction procedure begins by finding the event vertex. An energetic electron in $\mathrm{D}_{2} \mathrm{O}$ will travel approximately $0.45 \mathrm{~cm}$ per $\mathrm{MeV}$ [81]. The 5-15 MeV electrons of interest for solar neutrinos travel only a few centimeters before falling below the threshold for the emission of Čerenkov light. Therefore, we can effectively consider the light from a neutrino interaction to be emerging from a single point. To reconstruct the spatial position of an event, we rely upon the timing and spatial position of the PMTs which have detected light. The fitter used in this dissertation (the so-called Grid fitter, or ftg) does not make any assumptions about the angular distribution of the emitted light [82] to find the event position, allowing it to be effective at reconstructing events of various types, (such as neutrons, electrons, and $\beta-\gamma \mathrm{s}$ ) which can have very different angular distributions of light.

In order to reconstruct an event, the $(\mathrm{x}, \mathrm{y}, \mathrm{z})$ position and time $(\mathrm{t})$ of the event vertex must be determined. The fitter begins by performing search of the entire detector using a three-dimensional grid of points which are separated by 1.5 meters over a $10.5 \mathrm{~m}$ radius sphere. At each point, a log-likelihood function (which will be discussed below) is maximized with respect to the time of the event vertex. After this first grid search, which reduces the risk of getting stuck at a local maximum, the (x,y,z,t) of the best fit grid point are used as the starting point for a full maximization of the same log-likelihood function. In this second phase, all four parameters $(\mathrm{x}, \mathrm{y}, \mathrm{z}, \mathrm{t})$ are free to vary.

The log-likelihood function used in the maximization is a function of the residual time $\left(t_{\text {res }}\right)$, which is the PMT hit time $\left(t_{\text {hit }}\right)$ minus the fit event vertex time $\left(t_{f i t}\right)$ and minus the time it takes light to travel from the fit vertex $\left(r_{f i t}\right)$ to the PMT in question $\left(r_{p m t}(i)\right)$. 
Using the fact that light travels at a speed of $c / n$, where $n$ is the index of refraction averaged over the detector media, this can be written as

$$
t_{r e s}(i)=t_{\text {hit }}(i)-t_{f i t}(i)-\left|r_{f i t}-r_{p m t}(i)\right| n / c
$$

The time residual distribution should be approximately Gaussian centered around $t_{r e s}=0$. However, since there can be late PMT hits due to scattered and reflected light, there is a long tail on the $t_{r e s}>0$ side. There is also a flat background due to PMT noise hits. The $t_{r e s}$ probability distribution that is used by the fitter, $P\left(t_{r e s}\right)$ is plotted in Figure 3.1. With this probability distribution, the log-likelihood function which is maximized in the fits is:

$$
\log -\text { likelihood }=\sum_{i=1}^{\text {Nhits }} \log \left(P\left(t_{\text {res }}\left(i, r_{f i t}, t_{f i t}\right)\right)\right),
$$

where Nhits is the number of photo-multiplier tube hits in the event.

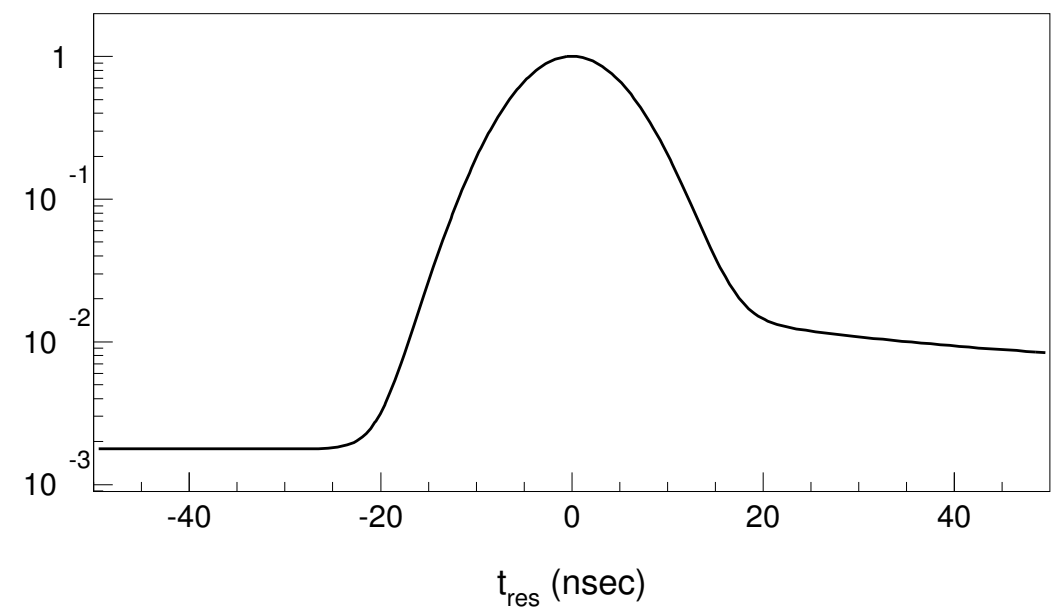

Figure 3.1: The probability distribution for $t_{r e s}$ that is used in event vertex reconstruction. The function shown here is for an event with 40 hits. 
Once the event vertex has been determined by the procedure outlined above, the fitter then determines the direction of the event. The concept of an event direction is only meaningful for a single particle event, such as a Čerenkov electron. To determine the direction, a probability density function (pdf) describing the Čerenkov cone of a 5 $\mathrm{MeV}$ electron is used [83]. The angular probability density for PMT hits, $P\left(\theta_{i}\right)$, is shown in Figure 3.2, where $\theta_{i}$ is the angle between the event direction and the PMT position (measured from the reconstructed event vertex). Again, the best fit angle is determined by maximizing the log-likelihood, given by:

$$
\log -\text { likelihood }=\sum_{i=1}^{\text {Nhits }} \log \left(P\left(\theta_{i}\right)\right) .
$$

Initially the likelihood is calculated for a grid of $\theta$ and $\phi$ values with a $10^{\circ}$ separation between the grid points. As is the case for the position reconstruction, the point with the largest likelihood in the grid search is then used as a starting point to find the $\theta$ and $\phi$ which maximizes the likelihood.

\subsection{Reconstruction Spatial Performance}

To evaluate the performance of the fitters, calibration data from several different sources was used. The primary source used to calibrate the Čerenkov response was a ${ }^{16} \mathrm{~N}$ source (described in [59]), which provides a tagged 6.13 MeV $\gamma$-ray. These calibrations are performed on a regular basis (approximately once a month). In the analysis presented here for the event reconstruction, all of the ${ }^{16} \mathrm{~N}$ source events were required to contain this tag. The ${ }^{16} \mathrm{~N}$ source is not strictly speaking a point source, since $\gamma$-rays will travel an average 


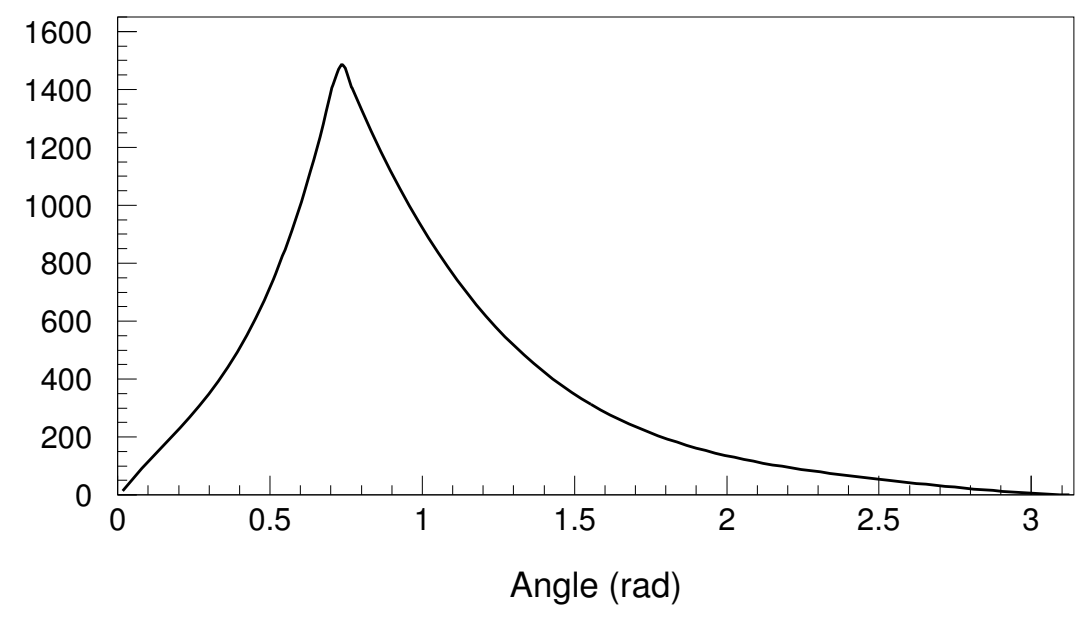

Figure 3.2: The probability density function for $\theta_{i}$ that is used for event direction reconstruction (the y-scale is arbitrary). The functional form is taken from [83] where it was determined by Monte Carlo simulations.

$\sim 40 \mathrm{~cm}$ from the source before Compton scattering off an electron. ${ }^{1}$

To calibrate the neutron response, a ${ }^{252} \mathrm{Cf}$ fission source is used. The neutrons produced by this source will randomly walk $1-2 \mathrm{~m}$ before capturing, so the ${ }^{252} \mathrm{Cf}$ source is also a rather diffuse source. Both the ${ }^{16} \mathrm{~N}$ source and ${ }^{252} \mathrm{Cf}$ source events were required to pass the instrumental backgrounds cuts which will be discussed in Chapter 7 .

Figure 3.3 shows the mean of the distance between the fit vertex and the center of the source, for both ${ }^{16} \mathrm{~N}$ data and Monte Carlo simulations at a variety of source positions in the $\mathrm{H}_{2} \mathrm{O}$ and $\mathrm{D}_{2} \mathrm{O}$. (This is the vector $r_{\text {dev }}$ in Figure 3.5.) Figure 3.4 shows a similar plot for data and Monte Carlo simulations from the ${ }^{252} \mathrm{Cf}$ source. The data and the Monte Carlo simulations show fairly good agreement on the resolution (to within $2 \mathrm{~cm}$ ). The

\footnotetext{
${ }^{1}$ Based on the equation in [84, Ch. 7], the Compton scattering cross section for $6.13 \mathrm{MeV} \gamma$-ray is roughly $7.21 \times 10^{-26} \mathrm{~cm}^{2}$, giving an interaction length of $41.5 \mathrm{~cm}$ in pure $\mathrm{D}_{2} \mathrm{O}$.
} 
largest discrepancy for the ${ }^{16} \mathrm{~N}$ data can be seen in a run which was taken near the neck (at $\mathrm{x}=0, \mathrm{y}=0, \mathrm{z}=+550)$. The values shown in Figures 3.3 and 3.4 do not reflect the true fitter resolution since the source is not a point source. Since the distribution of captures from the neutron source is inherently more diffuse that the $\gamma$-ray source, it is also not surprising that the $r_{d e v}$ is larger for events from the ${ }^{252} \mathrm{Cf}$ and the ${ }^{16} \mathrm{~N}$ source.

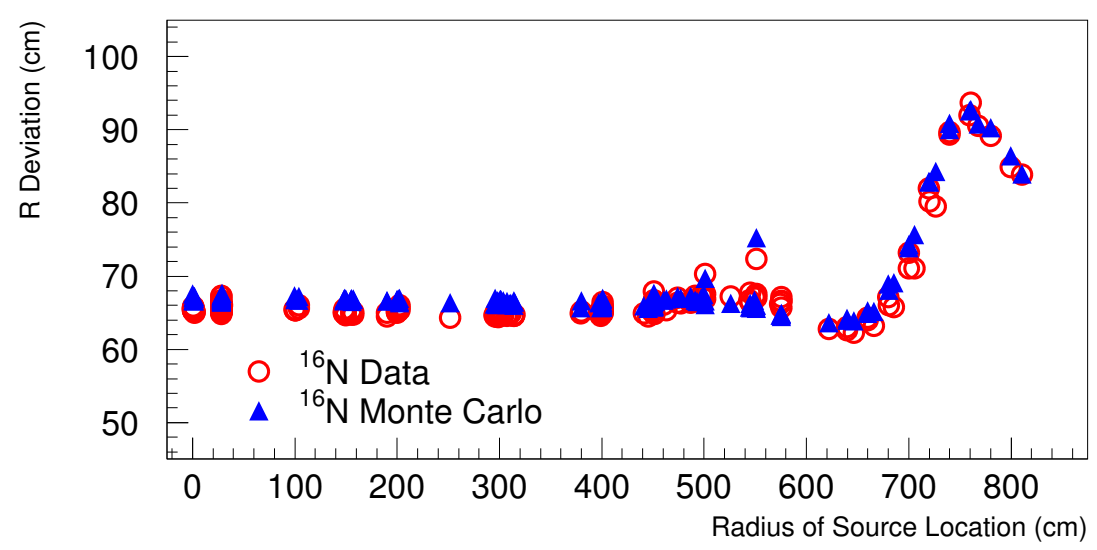

Figure 3.3: The mean distance between the source center and the reconstructed event vertices for ${ }^{16} \mathrm{~N}$ source data and Monte Carlo simulations, as a function of the source position in the detector. (This is the vector $r_{d e v}$ in Figure 3.5.)

Since the detector is spherical, the reconstructed radius is the most important measure of the position. We are primarily concerned with events coming from inside the $\mathrm{D}_{2} \mathrm{O}$, so in an analysis we can restrict ourselves to only looking at the inner part of the $\mathrm{D}_{2} \mathrm{O}$. A fiducial volume, which consists of only the events which are within $550 \mathrm{~cm}$ of the center of the detector, will be used for neutrino analysis. This is due to external backgrounds, such as "AV" events, external neutrons, and $\mathrm{H}_{2} \mathrm{O}$ radioactivity, which will be discussed in Chapter 8. Assuming the fitter response is symmetric, at the edge of the fiducial volume 


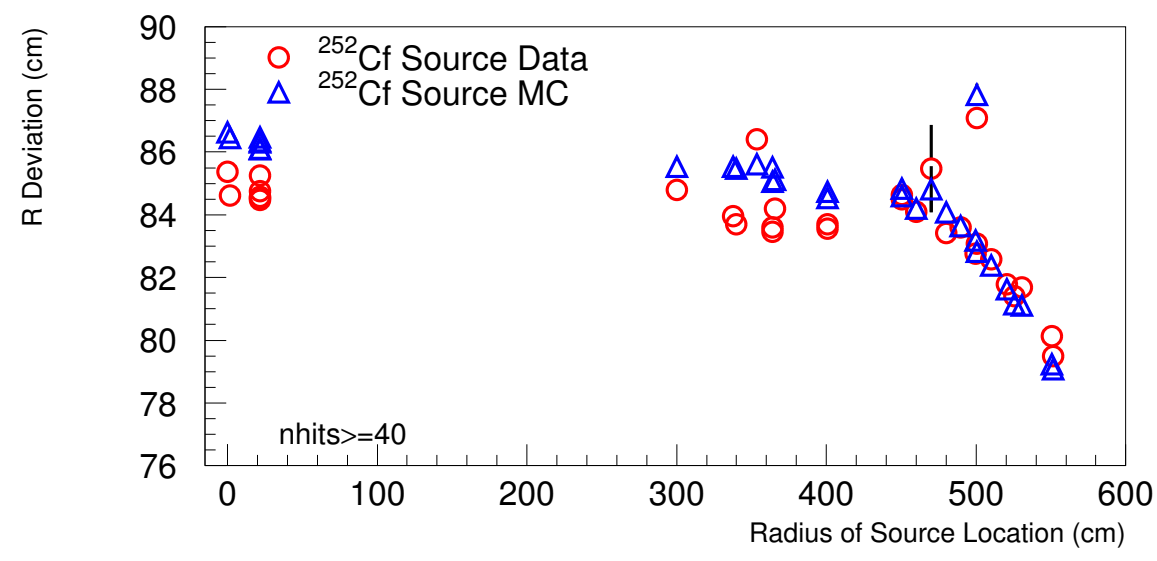

Figure 3.4: The mean distance between the source center and the reconstructed event vertices for ${ }^{252} \mathrm{Cf}$ source data and Monte Carlo simulations.

the fitter will push out the same number of events that it pulls in. Therefore the actual fitter resolution is of secondary importance compared to accuracy with which the fiducial volume can be determined. To understand the accuracy in determining the fiducial volume and to investigate if there is an overall tendency for the fitter to push events out or in, we can look at a parameter called fitter pull, which describes the tendency for a fitter to push or pull events radially. As is shown in Figure 3.5, $\mathrm{r}_{\text {pull }}$ is defined as the difference between the fit position and the source position projected along the radial vector to the center of the source. If the source distribution is symmetric and the fitter is not pushing out or pulling in events, then we would expect that the $\mathrm{r}_{\text {pull }}$ distribution should be symmetric around zero.

Figure 3.6 shows the mean fitter pull for ${ }^{16} \mathrm{~N}$ source data and Monte Carlo simulations. The mean was determined by fitting the distribution of events at each source location to a Gaussian. There is good agreement in the overall shape between the data and Monte 


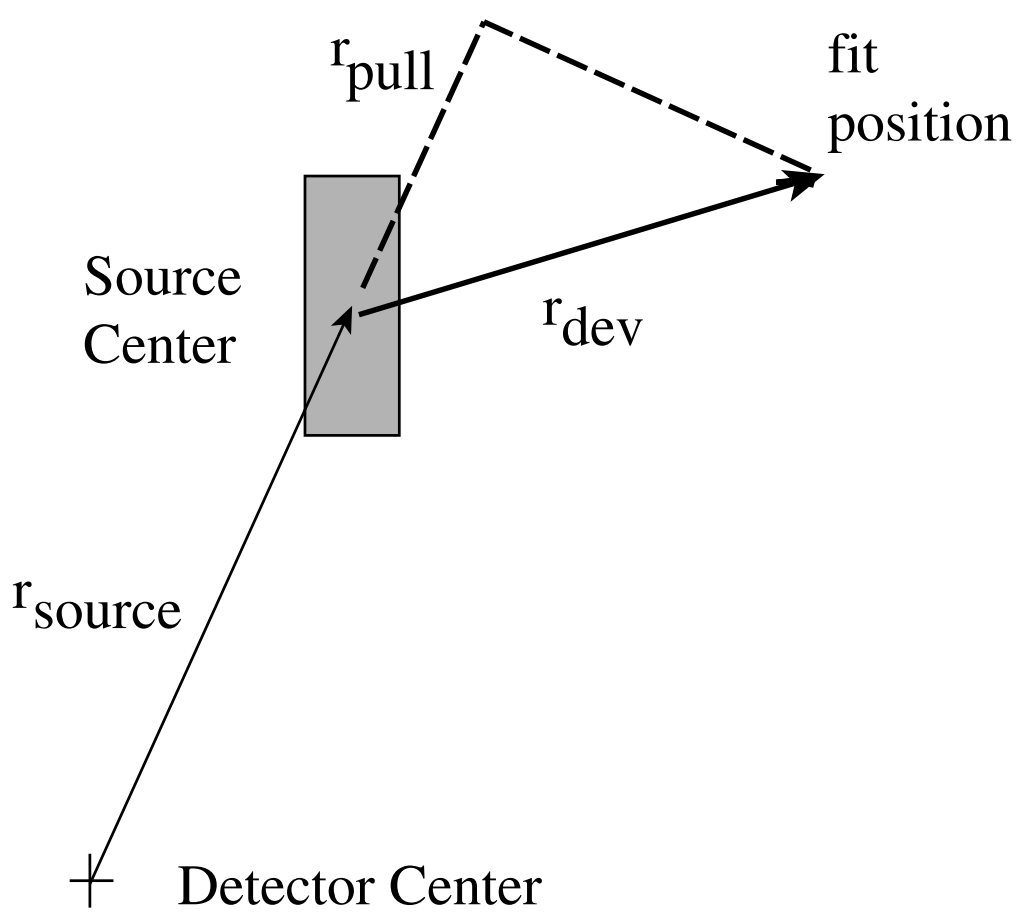

Figure 3.5: Fitter pull is defined as the difference between the source center position and reconstructed event vertex, projected along the radial vector to the center of the source. 
Carlo simulations. Much more scatter is seen in the data than the MC and this is due to the fact that our knowledge of the position of a deployed source in SNO is only accurate to 5-10 cm. A $5 \mathrm{~cm}$ inaccuracy in the position of the source could manifest itself as a $\pm 5 \mathrm{~cm}$ pull. Figure 3.7 shows a histogram of the mean radial pull for MC runs with the source in the $\mathrm{D}_{2} \mathrm{O}$ from 450 to $600 \mathrm{~cm}$. From this it can be seen that in the vicinity of the edge of the $\mathrm{D}_{2} \mathrm{O}$ the fitter pull in the radial direction is largely between +2 and $-2 \mathrm{~cm}$. Therefore, for the fiducial volume cut placed at $550 \mathrm{~cm}$, the overall uncertainty on the fiducial volume is $<2 \mathrm{~cm}$.

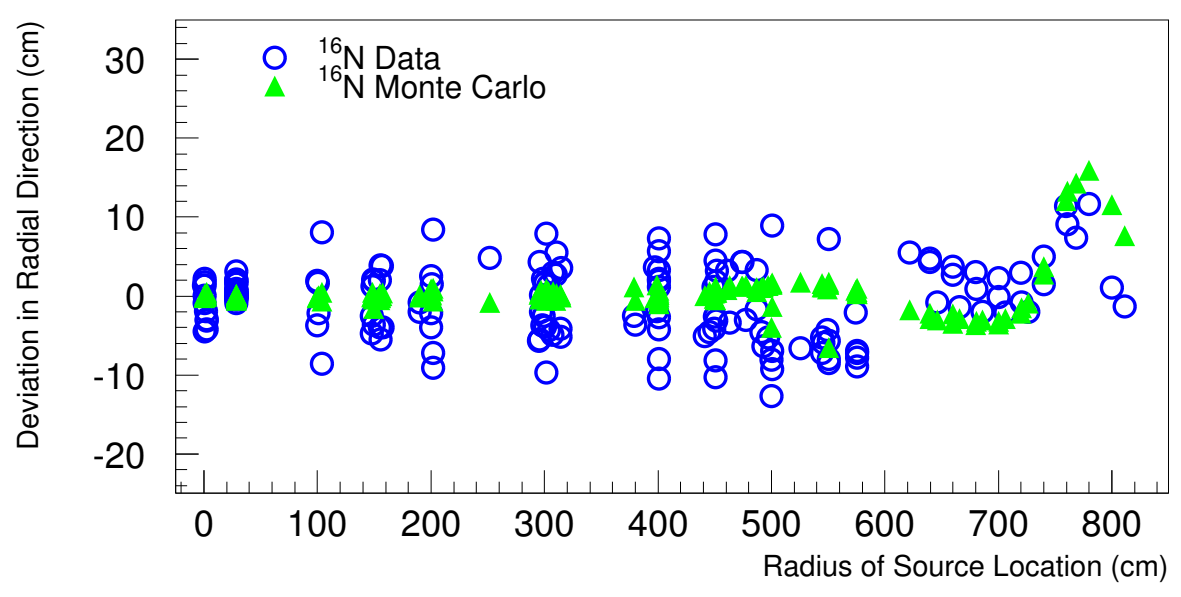

Figure 3.6: The mean fitter pull (determined by a gaussian fit) for data and Monte Carlo simulations from the ${ }^{16} \mathrm{~N}$ source.

We would also like to check that the fitter pull for neutron events is similar to that of electron events in order to ensure that we are sampling the same effective volume for all of our neutrino signals. It is not possible to study the fitter pull as a function of radius using neutron data since neutrons reaching the $\mathrm{AV}$ will capture on $\mathrm{H}$ and fall below 


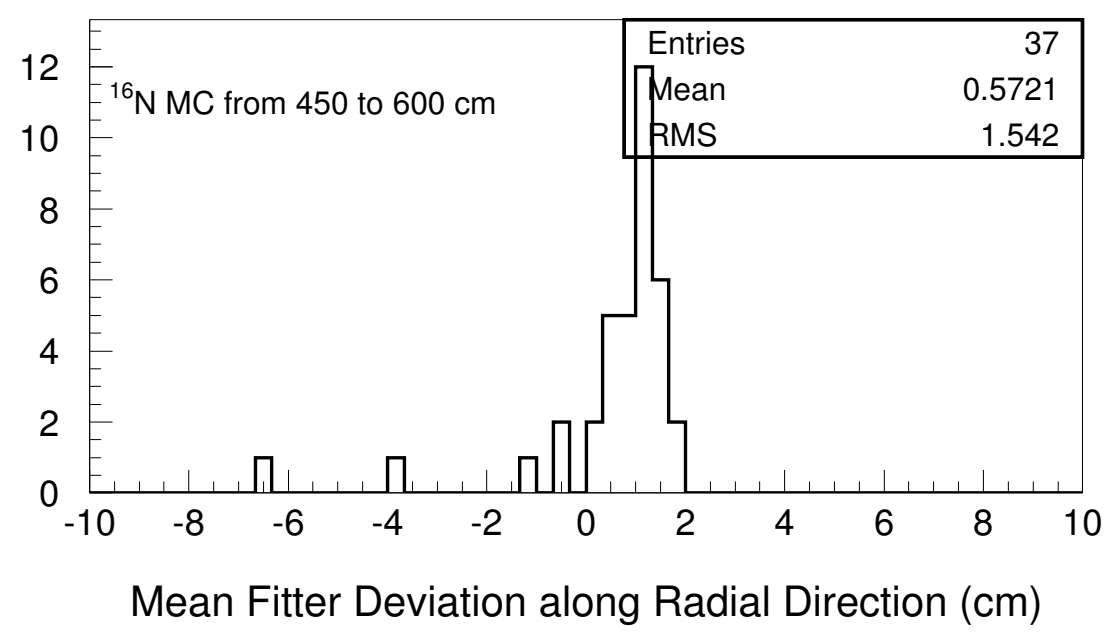

Figure 3.7: The mean fitter pull for ${ }^{16} \mathrm{~N}$ source MC for runs taken with the source in the $\mathrm{D}_{2} \mathrm{O}$ with the center of the source 450 to $600 \mathrm{~cm}$ from the center of the detector.

our detection threshold. Therefore, especially when the source is close to the AV, the radial distribution of the observed neutron events is not symmetric. On the other hand we can look at Monte Carlo simulations where we know the location of the neutron capture and see if there is trend among Monte Carlo simulated events to be pulled out or in with respect to their origin point. Figure 3.8 shows the pull along the radial direction for MC neutrons and MC $6 \mathrm{MeV}$ electrons (which have a similar light output to neutrons). Here the pull is based on the MC event position, rather than the center of the source (as was shown in Figure 3.5). For the neutrons, the MC capture position of the neutrons is used, while for the electrons the electron generation point is used. The agreement on the fitter pull between the neutron and electron MC events is good. Again the uncertainty on the radial position for events near $550 \mathrm{~cm}$ is roughly $2 \mathrm{~cm}$ for both neutrons and electrons. 


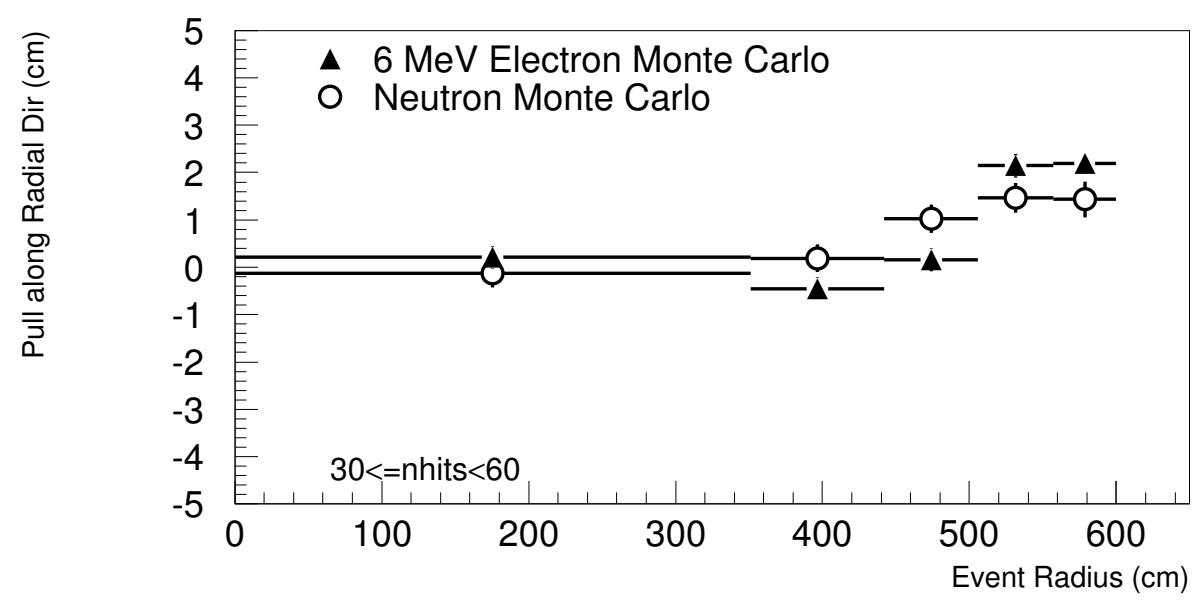

Figure 3.8: The fitter pull for electron and neutron MC events distributed throughout the $\mathrm{D}_{2} \mathrm{O}$. The pull here is based on the MC event position.

\subsection{Reconstruction Directional Performance}

To evaluate the performance of the event fitter in determining the event direction, the ${ }^{16} \mathrm{~N}$ source was used. Since the $\gamma$-rays will travel $\sim 40 \mathrm{~cm}$ before Compton scattering with an electron, then the vector between the event vertex and the source center gives us an indication of the initial particle direction. As is indicated in Figure 3.9, $\theta_{s}$, which is the angle between the event vertex and the reconstructed event direction, gives a measure of the fitter direction resolution. Only events which reconstruct more than $1 \mathrm{~m}$ from the center of the source are used. The distribution of $\cos \left(\theta_{s}\right)$, peaks at +1 . The value of $\cos \left(\theta_{s}\right)$ which encompasses $68 \%$ of the events between it and +1 is used to quantify the width of this distribution (see Figure 3.9). I will refer to this as the $68 \%$ value of $\cos \left(\theta_{s}\right)$.

Figure 3.10 gives the $68 \%$ values for ${ }^{16} \mathrm{~N}$ data and Monte Carlo simulations as a 


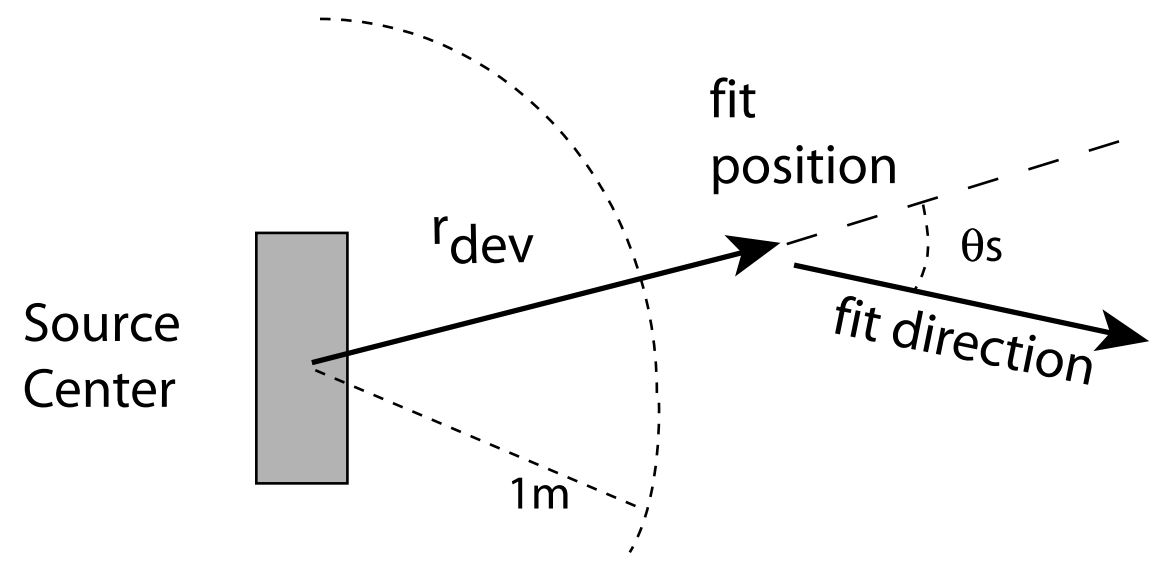

Histogram of $\cos (\theta s)$

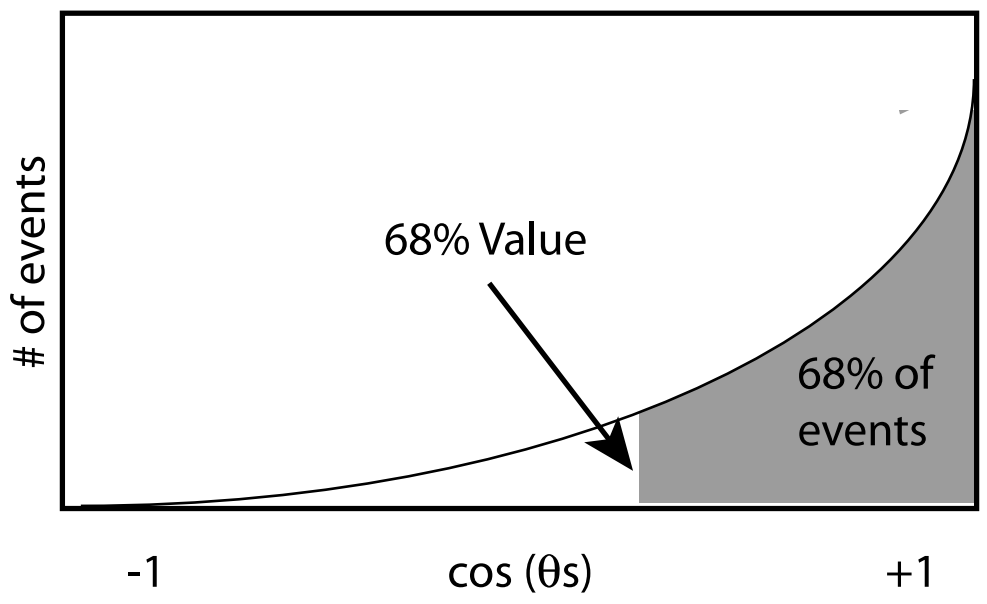

Figure 3.9: The top figure illustrates the definition of $\theta_{s}$. A typical $\cos \left(\theta_{s}\right)$ histogram is depicted in the lower plot. To quantify the narrowness of the distribution, the value which contains $68 \%$ of the event between it and +1 is used. 
function of the source location. Overall the agreement between the data and MC looks very good and the angular resolution remains relatively flat out to source positions within $500 \mathrm{~cm}$ of the center of the detector. Figure 3.11 shows the difference between the $68 \%$ values for the data and MC for runs in Figure 3.10 within $550 \mathrm{~cm}$ of the center. The average difference between the data and $\mathrm{MC}$ for the angular resolution is 0.007 (in $\cos \left(\theta_{s}\right)$ ), or roughly 0.8 degrees.

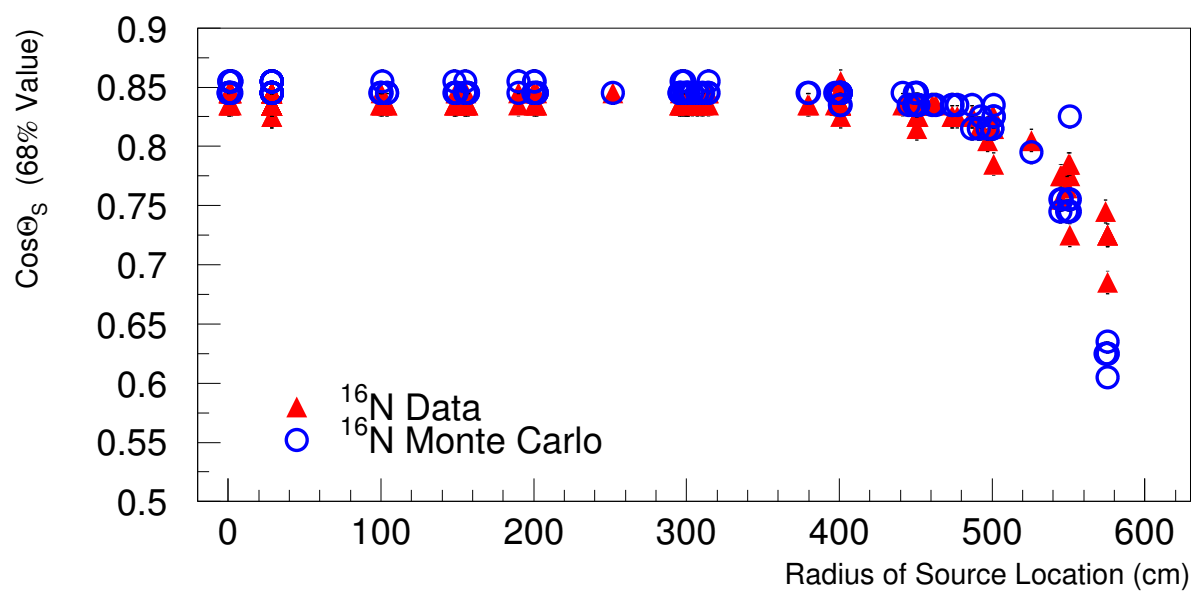

Figure 3.10: The $68 \%$ values for $\cos \left(\theta_{s}\right)$ values for ${ }^{16} \mathrm{~N}$ data and $\mathrm{MC}$ within the $\mathrm{D}_{2} \mathrm{O}$.

\subsection{Reconstruction Efficiency}

We also need to know the efficiency for successfully reconstructing an event. Here, we can take advantage of the fact that the ${ }^{16} \mathrm{~N}$ source is tagged, which allows us to select only events with a $\gamma$-ray. Figure 3.12, shows the fitter efficiency vs. the radius of the source 


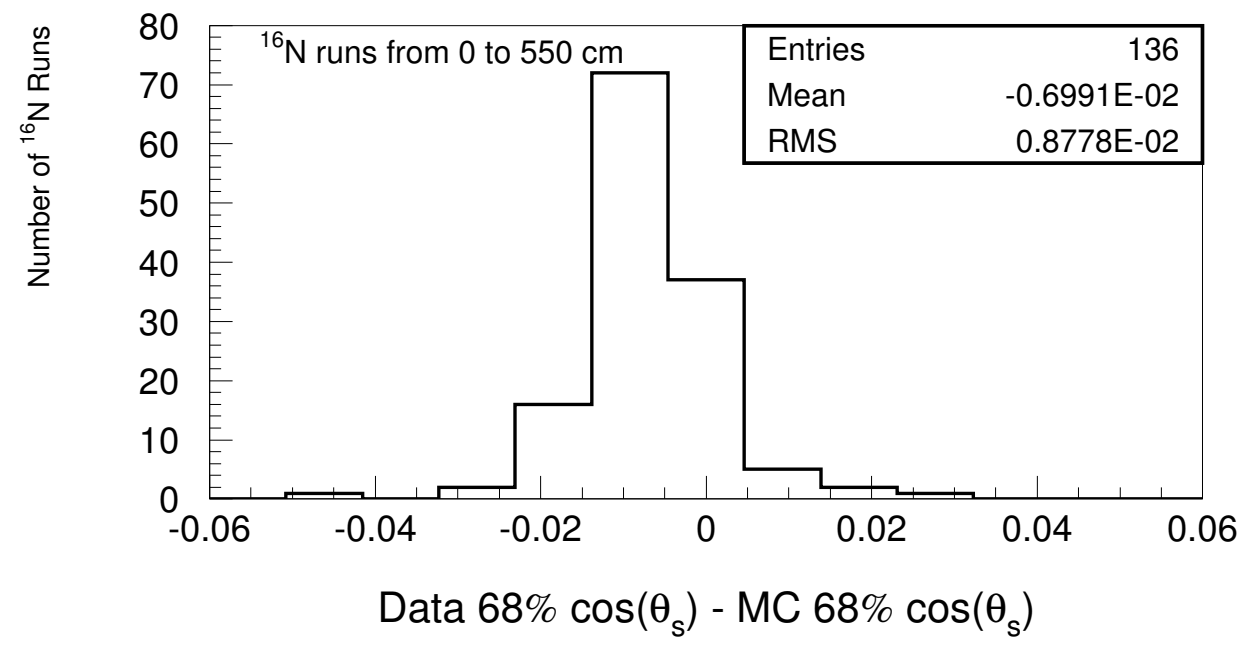

Figure 3.11: The difference between the data $68 \% \cos \left(\theta_{s}\right)$ value and the $\mathrm{MC} 68 \% \cos \left(\theta_{s}\right)$ value for ${ }^{16} \mathrm{~N}$ runs within $550 \mathrm{~cm}$ of the center.

location, with efficiency defined as

$$
\epsilon=\frac{\# \text { of tagged events with } 0<\text { fit radius }<1200 \mathrm{~cm}}{\# \text { of tagged events }} .
$$

Here the instrumental background cuts have not been applied to the data. The points in Figure 3.12 , have been fit to a function of the form $\epsilon=p 1-p 2(\exp (r / p 3))$, where $\mathrm{p} 1$, $\mathrm{p} 2$, and $\mathrm{p} 3$ are parameters determined by the fit. Averaging the resulting function over $r^{3}$ yields a fitter efficiency of $99.57 \pm 0.23 \%$ for events originating inside of $550 \mathrm{~cm}$. Note that this value represents the efficiency of the event being successfully reconstructed, and not the fraction of events which reconstruct within the fiducial volume. A similar procedure has also been applied to the Monte Carlo simulations (where $\epsilon$ is defined as the number of events reconstructing within $1200 \mathrm{~cm}$ over the total number of events), yielding a volumeweighted efficiency of $99.47 \pm 0.75 \%$ inside of $550 \mathrm{~cm}$, in good agreement with the value from 
data. Since both the data and the Monte Carlo simulations have a similar efficiency for reconstructing events, the relevant uncertainty on the neutrino fluxes is the uncertainty on the reconstruction efficiency, or $0.23 \%$.

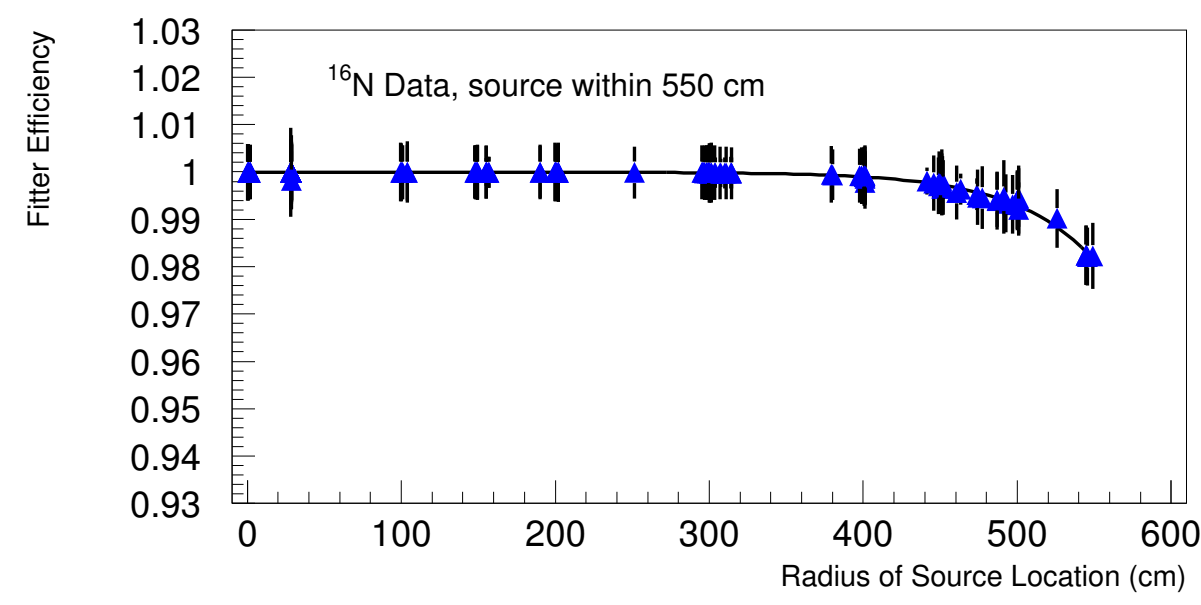

Figure 3.12: The fitter efficiency (defined in Equation 3.4) as a function of the source radial position. The line represents the function $\epsilon=1.000-4.3 \times 10^{-7}\left(e^{r / 51.6}\right)$.

\subsection{Summary of Reconstruction}

Table 3.1 summarizes the uncertainties due to event position and direction reconstruction. For the CC and ES, the values were obtained from the ${ }^{16} \mathrm{~N}$ source data and MC and the NC values generally come from the ${ }^{252} \mathrm{Cf}$ source data and MC. For the reconstruction efficiency and angular resolution only the ${ }^{16} \mathrm{~N}$ source was used, so the value it yielded has been assumed to hold for all three types of signals. 


\begin{tabular}{|c||c|c|c|}
\hline \hline Source & CC & ES & NC \\
\hline \hline Radial Pull & $<2 \mathrm{~cm}$ & $<2 \mathrm{~cm}$ & $<2 \mathrm{~cm}$ \\
\hline Data and MC Agreement on $\cos \left(\theta_{s}\right)$ & 0.007 & 0.007 & 0.007 \\
\hline Reconstruction Efficiency Uncertainty & $0.23 \%$ & $0.23 \%$ & $0.23 \%$ \\
\hline
\end{tabular}

Table 3.1: Reconstruction systematics within $550 \mathrm{~cm}$ 


\section{Chapter 4}

\section{Energy Calibration}

\subsection{Energy Estimator based on ${ }^{16} \mathrm{~N}$ Source Calibration}

As described in Chapter 2, SNO has a variety of calibration sources. The primary source for the energy calibration of the SNO detector is the ${ }^{16} \mathrm{~N}$ source, which provides tagged 6.13 $\mathrm{MeV} \gamma$-rays. The ${ }^{16} \mathrm{~N}$ source was deployed at the center of the detector on a regular basis over the time period spanning the official salt phase data. There were also a number of scans of the detector performed at various off-center positions in both the $\mathrm{H}_{2} \mathrm{O}$ and $\mathrm{D}_{2} \mathrm{O}$.

\subsubsection{Number of hits on working PMTs: $n_{\text {whits }}$}

The principal means of estimating the energy for an event in SNO is to count up the number of photo-multiplier tubes that fired within the trigger window. However, there are a number of corrections which must be applied to this. Over the lifetime of the detector, some photo-multiplier tubes have stopped functioning and at various times 


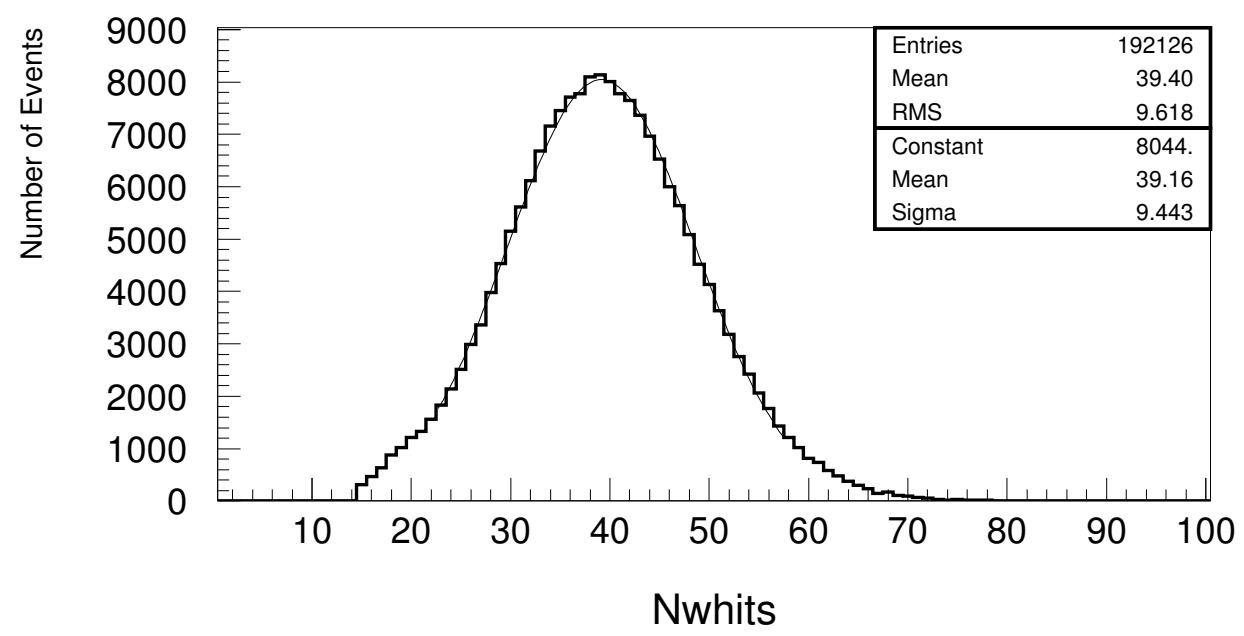

Figure 4.1: Distribution of $n_{\text {whits }}$ for a single ${ }^{16} \mathrm{~N}$ source run at the center of the detector. It has been fit to a Gaussian.

particular electronics cards may be temporarily offline. So, we should start by counting up only the hits on tubes which are believed to be working. This quantity will be referred to as $n_{w h i t s}$. Figure 4.1 shows a plot of $n_{\text {whits }}$ for a single run at the center of the detector. It is fairly Gaussian in shape. However, due to the presence of $7.11 \mathrm{MeV} \gamma$-rays in $4.8 \%$ of ${ }^{16} \mathrm{~N}$ decays, some deviations from Gaussian are expected. As was the case in the previous chapter, the ${ }^{16} \mathrm{~N}$ data events here are required to have a tag and to pass the instrumental background removal cuts which will be described in Chapter 7 .

\subsubsection{Noise and Tubes Online Correction}

Ultimately we would like an energy estimator to be stable with respect to time and not to have a bias with event position or direction. To achieve this, there are a number of corrections that must be applied to $n_{\text {whits }}$. Firstly, we must subtract off noise hits. For 
the SNO PMTs, the mean noise rate in a SNO PMT is approximately $500 \mathrm{~Hz}$ [50]. So, for 9000 photo-multiplier tubes there will be approximately 1.8 noise hits per event in a 400 nsec trigger window. As indicated in Chapter 2, the SNO trigger system has a pulsed global trigger which "captures" the state of the detector every $200 \mathrm{msec}$, giving an idea of the average number of noise hits. For each individual data run, the average number of hits in the pulsed trigger events has been calculated and this value is subtracted from $n_{\text {whits }}$. This noise rate is also included in Monte Carlo simulations, so it will also be subtracted from Monte Carlo simulated events.

Since the number of operating photo-multiplier tubes is changing over time, it is also necessary to correct for the number of online photo-multiplier tubes for a given run. At the commencement of production data for the salt phase, a number of ${ }^{16} \mathrm{~N}$ runs were taken. All of the subsequent data will be normalized to match the energy response of the detector on that day (July 26, 2001 or modified Julian day 9705), at which point there were 9115 PMTs online. ${ }^{1}$

\subsubsection{Date Correction}

Figure 4.2 shows a plot of the mean $n_{\text {whits }}$ (with the noise subtracted and the correction for the number of online photo-multiplier tubes applied) for the ${ }^{16} \mathrm{~N}$ source data at the center of the detector at various dates. Here the mean for each run has been determined by a fit to a Gaussian. Though the data has been corrected for the number of tubes online, there is still an overall decrease in the energy response over time. This is believed to be

\footnotetext{
${ }^{1} \mathrm{SNO}$ uses a modified Julian date, inherited from the Soudan2 astronomy libraries, defined as days since 12:00 (in Greenwich) on December 31, 1974. Throughout this dissertation, this will be referred to simply as Julian Date.
} 
due to an increase in the optical attenuation of the $\mathrm{D}_{2} \mathrm{O}$, which has also been observed in the optical measurements [85]. During the salt phase, some of the water purification systems for the $\mathrm{D}_{2} \mathrm{O}$, such as the reverse-osmosis unit, could not be used since they would have removed some of the salt. Over time there was a build up of impurities in the $\mathrm{D}_{2} \mathrm{O}$, especially $\mathrm{Mn}$ from the water radioassays which use $\mathrm{MnOx}$ coated beads. The last $\mathrm{MnOx}$ assay was performed in April 2003 (Julian Day 10332) and the salt was removed on Sept. 8, 2003 (Julian Day 10478). As can be seen in Figure 4.2, the slope of the energy drift becomes flatter after the last $\mathrm{MnOx}$ assay. Once the salt was removed, the energy response of the detector returned back to where it was at the start of the salt phase, further supporting the idea that impurities building up in the $\mathrm{D}_{2} \mathrm{O}$ were largely responsible for the energy drift with time.

In the MC simulations, some of the optical inputs have been varied as a function of time to echo the behavior seen in the data. At the present moment, the Monte Carlo simulation does not know that the salt was removed on Julian Day 10478, so the energy scale does not bounce back the way that it does in the data. As this dissertation focusses on the salt phase data (up to Julian Day 10478) this is not important. Figure 4.3 shows a plot of the mean $n_{\text {whits }}$ for ${ }^{16} \mathrm{~N}$ MC with corrections for PMT noise and the number of tubes online.

To correct for the remaining decrease in the light response over time, both the data and Monte Carlo simulations have been fit to lines (see Figures 4.2 and 4.3), and these lines were then used to normalize the energy scale to match the response at the start of the salt dataset. Here both the data and Monte Carlo simulations have been split into two 
regions: one for the data from Julian Day 9704 to 10332 (the date of the last MnOx assay), and to another after Julian Day 10332. The time correction functions for the data and MC are:

Region 1 (Julian Day 9704 to 10332):

$$
\begin{aligned}
& \text { Tcor }_{\text {data }}=59.44( \pm .17)-0.00209( \pm 0.00002) \times \text { Julian Day } \\
& \text { Tcor }_{m c}=60.01( \pm .17)-0.00223( \pm 0.00002) \times \text { Julian Day }
\end{aligned}
$$

Region 2 (Julian Day 10333 to end ):

$$
\begin{aligned}
& \text { Tcor }_{\text {data }}=49.45( \pm .39)-0.00111( \pm 0.00004) \times \text { Julian Day } \\
& \text { Tcor }_{m c}=46.96( \pm .47)-0.00096( \pm 0.00005) \times \text { Julian Day }
\end{aligned}
$$

Taking into account the correlations between the parameters, the uncertainty on the data time scale in Region 1 is roughly $0.03 \%$ and is roughly $0.06 \%$ in Region 2 . We can conservatively take $0.06 \%$ to be the uncertainty on the time dependence of the energy scale.

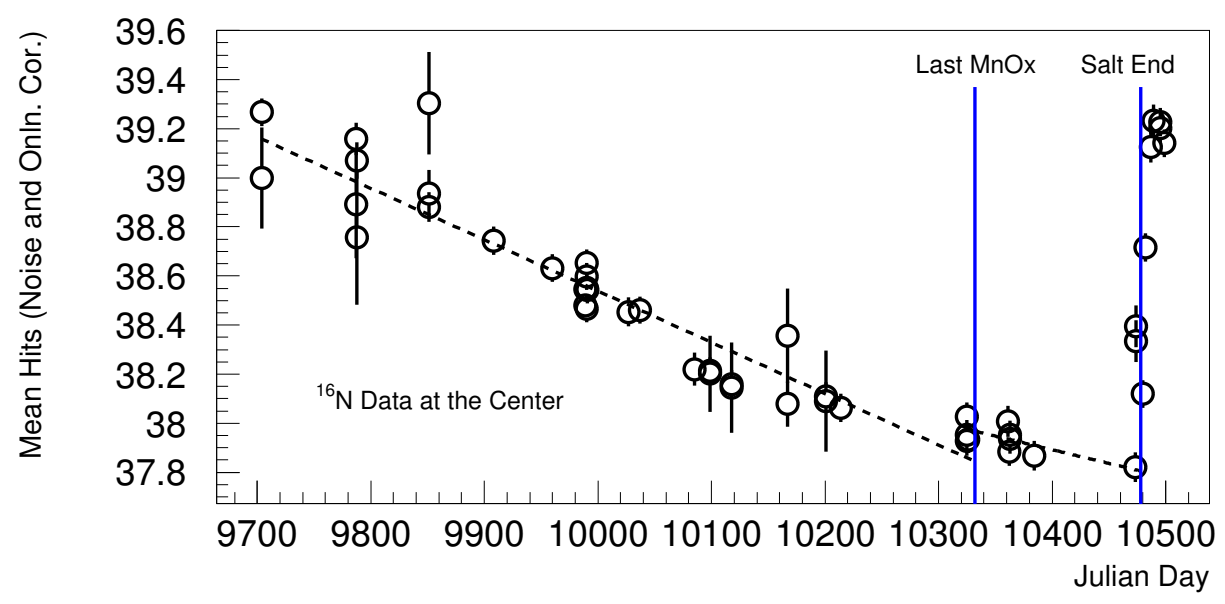

Figure 4.2: The mean $n_{\text {whits }}$ with the average noise level subtracted and corrected for the number of photo-multiplier tubes online for ${ }^{16} \mathrm{~N}$ source data. 
Figure 4.4 shows the ${ }^{16} \mathrm{~N}$ data and Monte Carlo simulations for runs at the center of the detector with Tcor applied. This correction to the data and the Monte Carlo simulations now gives a relatively flat response with respect to Julian Day, though an overall shift between the data and Monte Carlo simulations is visible.

\subsubsection{Radial Correction}

So far, only the energy response for runs taken at or near the center of the detector has been considered. However, there is also a variation in the energy response with respect to radius. Figure 4.5 shows the mean $n_{\text {whits }}$ (noise, online, and Tcor corrected) for ${ }^{16} \mathrm{~N}$ data and Monte Carlo simulated events plotted against $(r / 600)^{3}$. (Note that $(r / 600)^{3}$ is $<1$ for events inside the $\mathrm{D}_{2} \mathrm{O}$ and $>1$ for events in the $\mathrm{H}_{2} \mathrm{O}$.) Here the events in the $\mathrm{D}_{2} \mathrm{O}$ have been fit to a third-order polynomial and the events in the $\mathrm{H}_{2} \mathrm{O}$ have been fit to a second-order

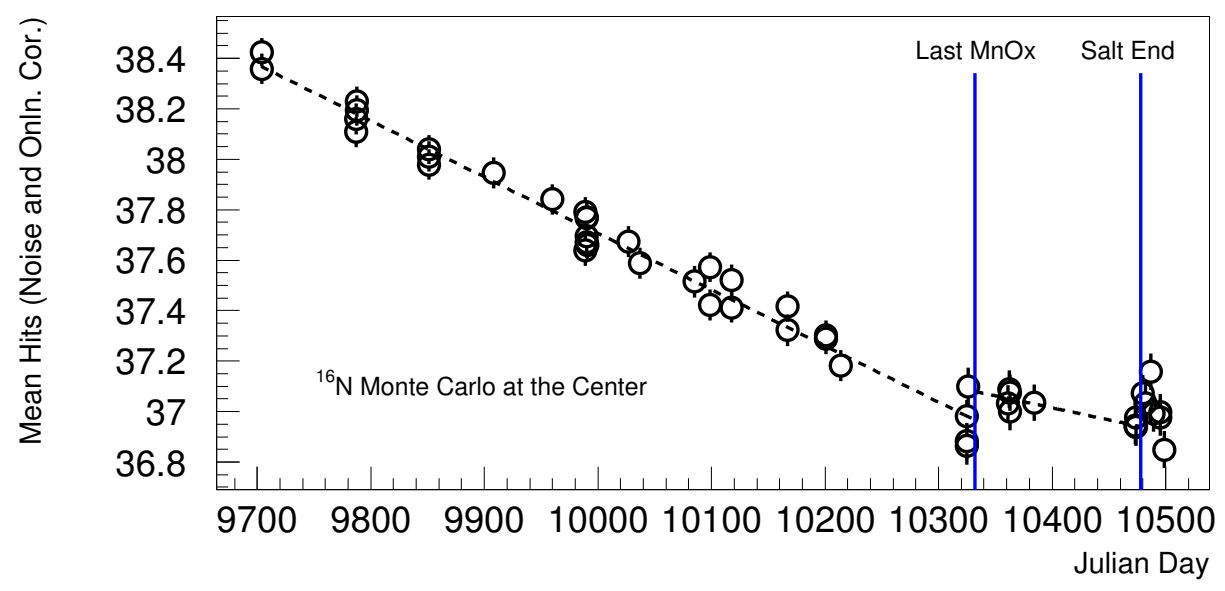

Figure 4.3: The mean of $n_{w h i t s}$ with the average noise level subtracted and corrected for the number of tubes online for ${ }^{16} \mathrm{~N}$ source MC. 


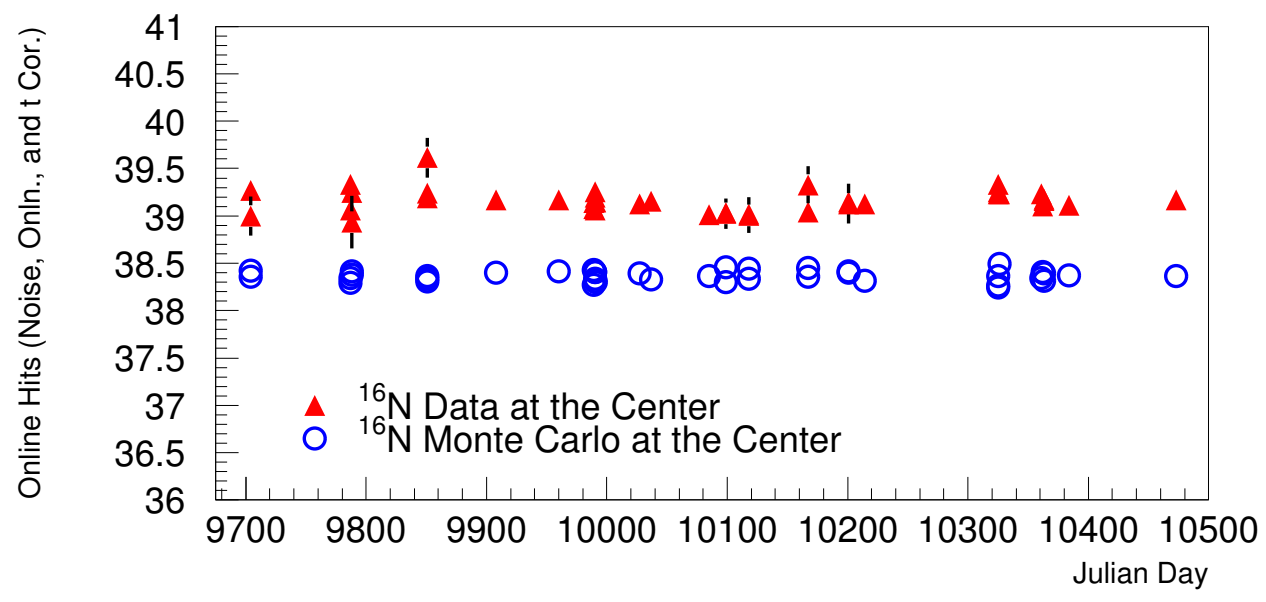

Figure 4.4: The mean $n_{w h i t s}$ corrected for average noise level, the number of tubes online and time corrected by the Tcor factor (Equation 4.1), plotted as a function of date.

polynomial. Defining $r 3=\left(r_{f i t} / 600\right)^{3}$, the functions describing the radial dependence in the $\mathrm{D}_{2} \mathrm{O}$ are

$$
\begin{aligned}
& \operatorname{Rcor}_{\text {data }}(r 3)=38.92+15.0 \times r 3-32.3 \times\left(r 3^{2}\right)+17.0 \times\left(r 3^{3}\right) \\
& \operatorname{Rcor}_{m c}(r 3)=38.08+11.9 \times r 3-23.2 \times\left(r 3^{2}\right)+11.0 \times\left(r 3^{3}\right),
\end{aligned}
$$

while the functions describing the radial dependence in the $\mathrm{H}_{2} \mathrm{O}$ are

$$
\begin{aligned}
& \operatorname{Rcor}_{d a t a}(r 3)=-11.6+78.9 \times r 3-28.41 \times\left(r 3^{2}\right) \\
& \operatorname{Rcor}_{m c}(r 3)=5.5+50.7 \times r 3-19.46 \times\left(r 3^{2}\right)
\end{aligned}
$$

To account for the fact that there is an overall difference of 0.84 hits between the response of the data and $\mathrm{MC}$ at the center, an additional scale factor of $M C_{\text {scale }}=$ $38.92 / 38.08=1.022$ is additionally applied to the Monte Carlo simulations. 


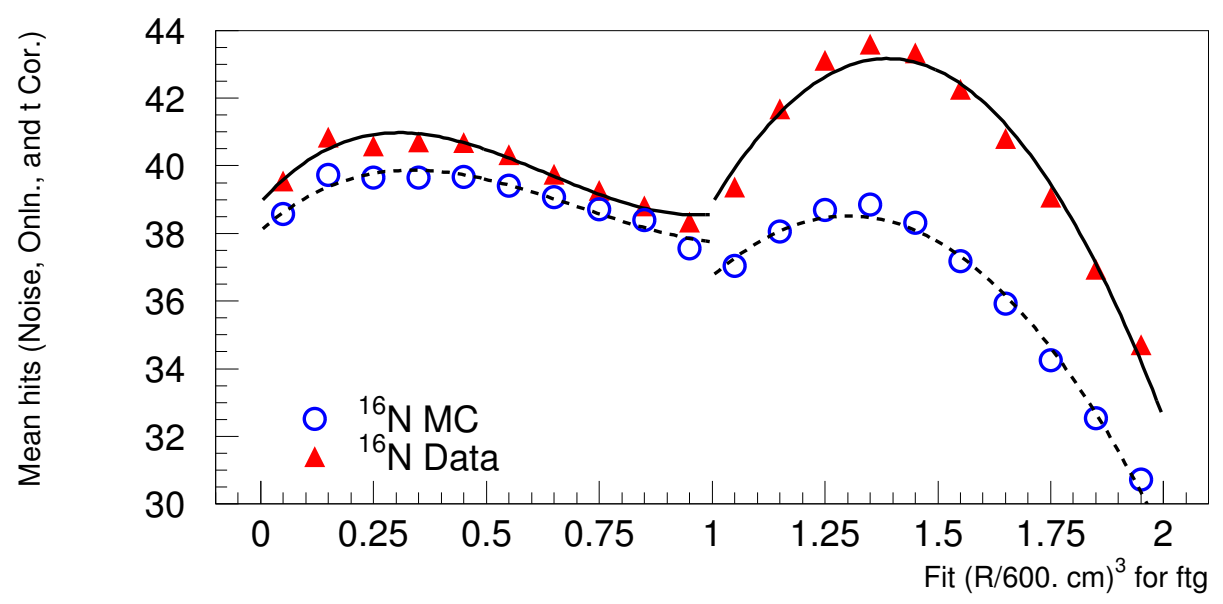

Figure 4.5: The mean $n_{\text {whits }}$ corrected for average noise level, the number of tubes online and Tcor, plotted against fit radius. For $\left.(r / 600)^{3}\right)<1$, the data and MC have been fit to a third-order polynomial and a second-order polynomial for $\left.(r / 600)^{3}\right)>1$.

\subsubsection{Number of effective PMT hits: $n_{e f f}$}

Putting together all of these corrections, we can write down the final expression for $n_{e f f}$, which is the effective number of hits, corrected for time, radius, noise hits, and the number of tubes online. For the data the expression is

$$
\begin{aligned}
n_{e f f}= & \left(n_{\text {whits }}-\# \text { noise hits }\right) \times \frac{9115}{\text { tubes online }} \\
& \times \operatorname{Tcor}_{\text {data }}(\text { Julian Day }) \times \operatorname{Rcor}_{\text {data }}(r 3),
\end{aligned}
$$

while for Monte Carlo simulations the corresponding expression is

$$
\begin{gathered}
n_{e f f}=\left(n_{\text {whits }}-\# \text { noise hits }\right) \times \frac{9115}{\text { tubes online }} \\
\times \operatorname{Tcor}_{m c}(\text { Julian Day }) \times \operatorname{Rcor}_{m c}(r 3) \times M C_{\text {scale }} .
\end{gathered}
$$


Figure 4.6 shows the resulting $n_{\text {eff }}$ plotted against the fit radius for ${ }^{16} \mathrm{~N}$ data and Monte Carlo simulations. For events within $556 \mathrm{~cm}$ of the center of the detector (the first 8 bins) the variation of the data as a function of fit radius is \pm 0.22 hits $(0.6 \%)$ and the data and the Monte Carlo simulations agree with each other to within \pm 0.1 hits (or $0.26 \%$ ).

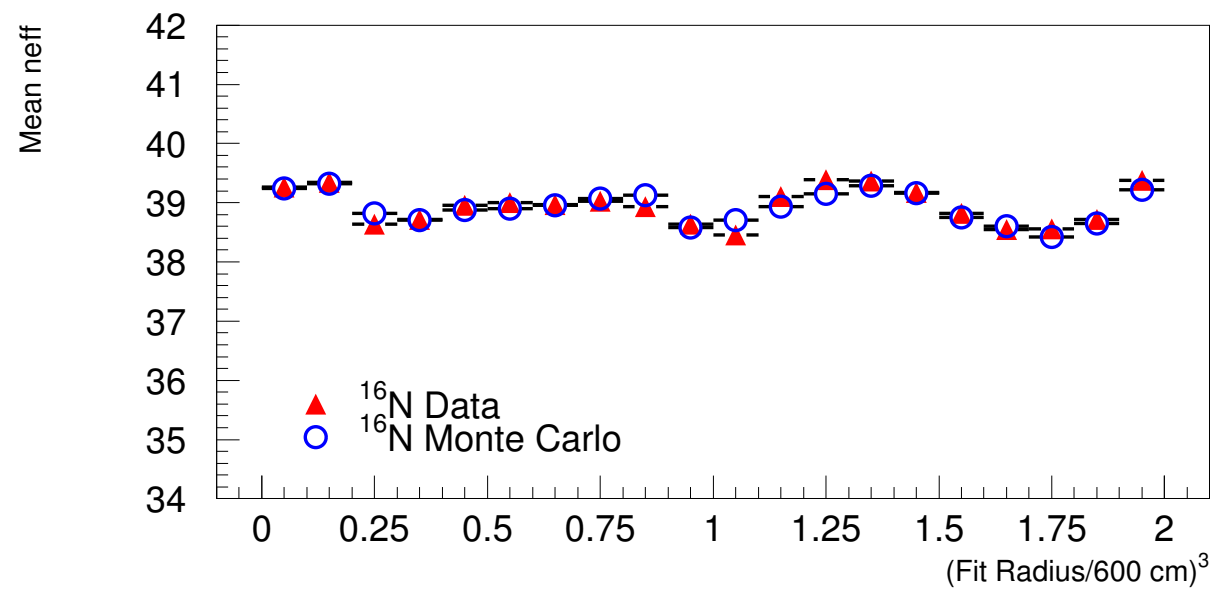

Figure 4.6: The mean $n_{\text {eff }}$ plotted against fit radius. The $\mathrm{MC}$ has also been corrected by $M C_{\text {scale }}$.

\subsection{Verification of Energy Scale with ${ }^{252} \mathrm{Cf}$ and AmBe Sources}

In the previous section, an energy estimator, $n_{e f f}$, was developed. It was also tuned so that the means of the ${ }^{16} \mathrm{~N}$ data and Monte Carlo simulation responses match. We would like to verify the agreement between the data and the Monte Carlo simulation with additional calibration sources.

Since the ${ }^{16} \mathrm{~N}$ source more closely resembles single electron events, we should ex- 
amine the energy response for neutron events. For the largest number of neutron statistics and source positions, we can look at the data from the ${ }^{252} \mathrm{Cf}$ source. Unfortunately this is not a tagged source. However we can apply some cuts to obtain a fairly pure sample of neutrons. The neutrons from ${ }^{252} \mathrm{Cf}$ are emitted in fission bursts. Prompt $\gamma$-rays associated with the fission are also emitted. To look for neutrons, bursts of 3 to 10 events within 15 msec of each other were located. A new burst could not begin until 40 msec after the first burst. Since the ${ }^{252} \mathrm{Cf}$ source fission rate is approximately $4 \mathrm{~Hz}$ [86], pile-up between subsequent fissions will be small. Events were also required to reconstruct within $675 \mathrm{~cm}$ of the center of the detector. Since the first event of a burst can be a signal from prompt $\gamma$-rays, the first event in each burst was eliminated.

Figure 4.7 shows a plot of $n_{\text {eff }}$ for neutron events from ${ }^{252} \mathrm{Cf}$ source data and Monte Carlo simulations as a function of the reconstructed events radius. The data and Monte Carlo simulations agree to within \pm 0.4 hits $(0.8 \%)$.

Since ${ }^{16} \mathrm{~N}$ and ${ }^{252} \mathrm{Cf}$ source events have a similar energies, it is also a good idea to check the energy response of the Monte Carlo simulation for events at a different energy. Here we can utilize the AmBe source. Inside the AmBe source, neutrons are produced via the ${ }^{9} \mathrm{Be}+\alpha \rightarrow n+{ }^{12} \mathrm{C}$ reaction. In roughly $59 \%$ of the decays, a $4.438 \mathrm{MeV} \gamma$-ray is produced in conjunction with a neutron [61]. Since neutrons typically take about 5 msec to capture, the $\gamma$-ray will appear in the data stream before the neutron. One can tag neutron events in the dataset by requiring the events to have between 45 and 80 hits (which is above most radioactive backgrounds and the $4.438 \mathrm{MeV} \gamma$-rays), to reconstruct less than $200 \mathrm{~cm}$ from the center of the source, and to have a large degree of isotropy in the distribution of 


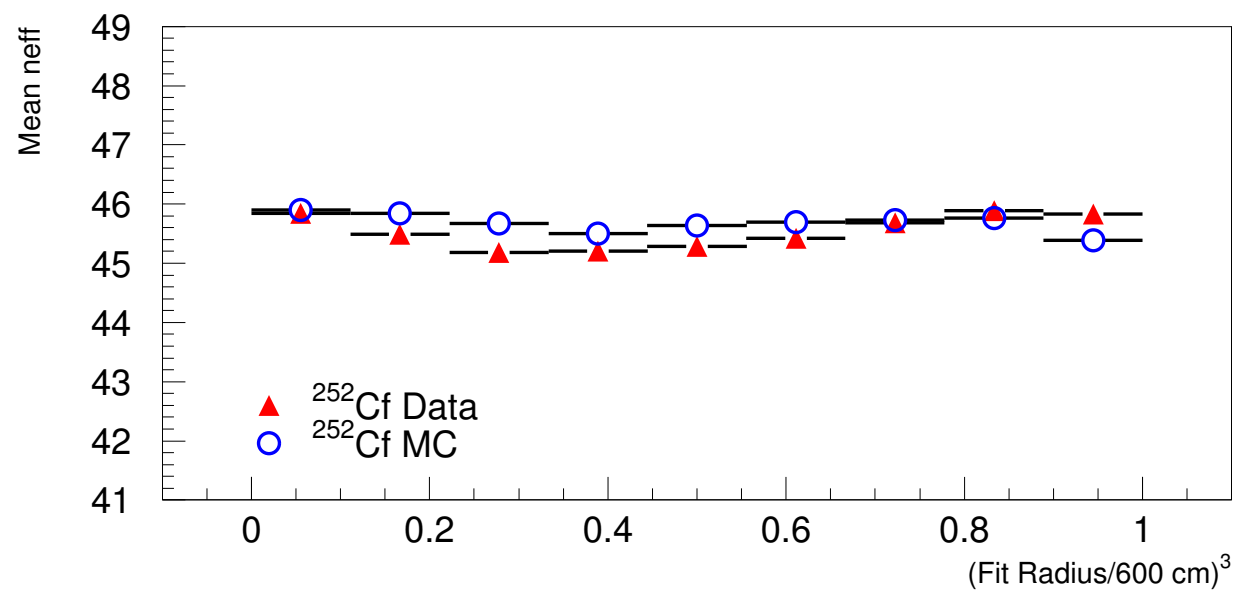

Figure 4.7: The mean $n_{\text {eff }}$ for ${ }^{252} \mathrm{Cf}$ data and $\mathrm{MC}$ as a function of fit radius.

light from the event (See Chapter 5). For each event, one can then search for the closest event before and after it. Looking at events which are 1 to $10 \mathrm{msec}$ before a neutron (possible $4.438 \mathrm{MeV} \gamma$-rays), and subtracting events which are 1 to $10 \mathrm{msec}$ after a neutron (a background sample), gives an energy distribution for $4.438 \mathrm{MeV} \gamma$-rays. Figure 4.8 shows the $n_{\text {eff }}$ distribution for data and Monte Carlo simulations for the $4.438 \mathrm{MeV} \gamma$-rays from several AmBe source runs summed together. The reason for the discrepancy on the low side is that the detector trigger threshold for these runs was 18 hits. When fit to Gaussians above $n_{\text {eff }}=18$, the mean $n_{\text {eff }}$ of the data is $24.92 \pm 0.07$ hits and the mean $n_{\text {eff }}$ for the $\mathrm{MC}$ is $25.09 \pm 0.02$ hits. The energy scale of the data and MC agree to within $0.7 \%$. 


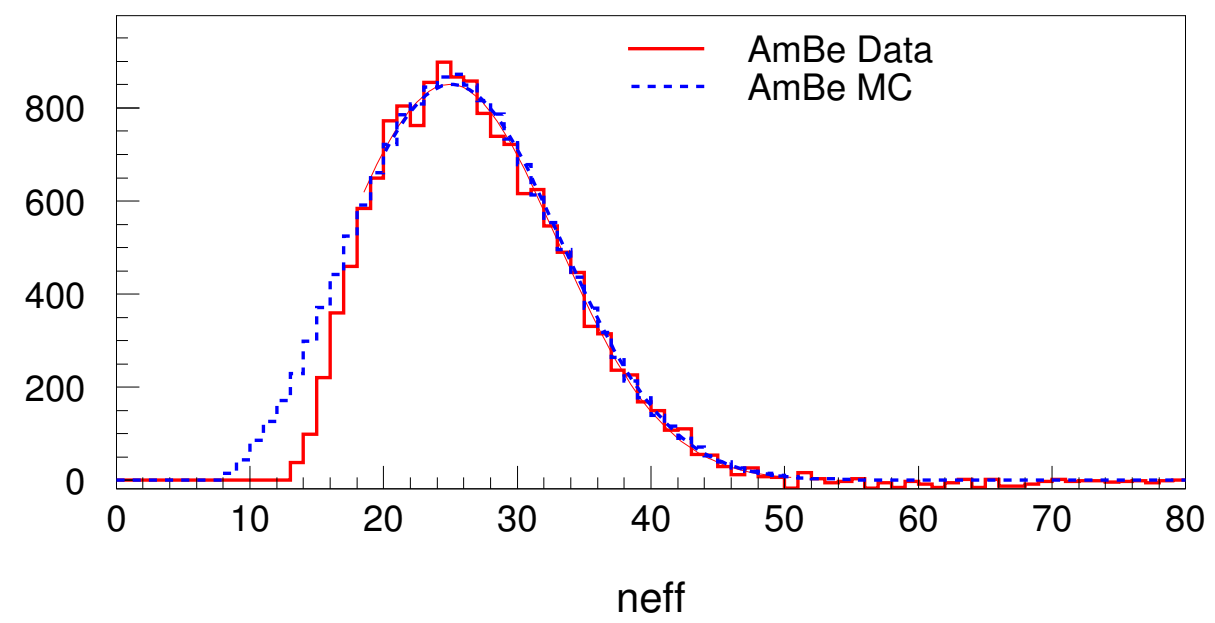

Figure 4.8: The mean $n_{\text {eff }}$ for several AmBe data and MC runs added together. The data was taken with a threshold of 18 hits, while the Monte Carlo has no threshold requirement.

\subsection{Energy vs. Angle}

Anther concern is the variation of the energy scale as a function of the direction of the event. Figure 4.9 shows the mean $n_{e f f}$ for events from several ${ }^{16} \mathrm{~N}$ runs at the center of the detector plotted as a function of the fit direction. The energy response of the detector is not completely uniform, but since the energy scale is determined by averaging over all directions, this is only a concern if the events from a particular signal type tend to travel in one direction more than another. For the NC events, which are distributed uniformly in all directions this is not a factor. However the ES and CC signals, whose event directions are related to the location of the Sun, will not sample the detector uniformly. To evaluate this, ES and CC Monte Carlo simulation files were generated with the same solar zenith angle and livetime distribution as the salt data. Using the response of the ${ }^{16} \mathrm{~N}$ data, the average 
shift in the energy compared to a uniform distribution was found to be $0.004 \%$ for the CC and $-0.06 \%$ for the ES.

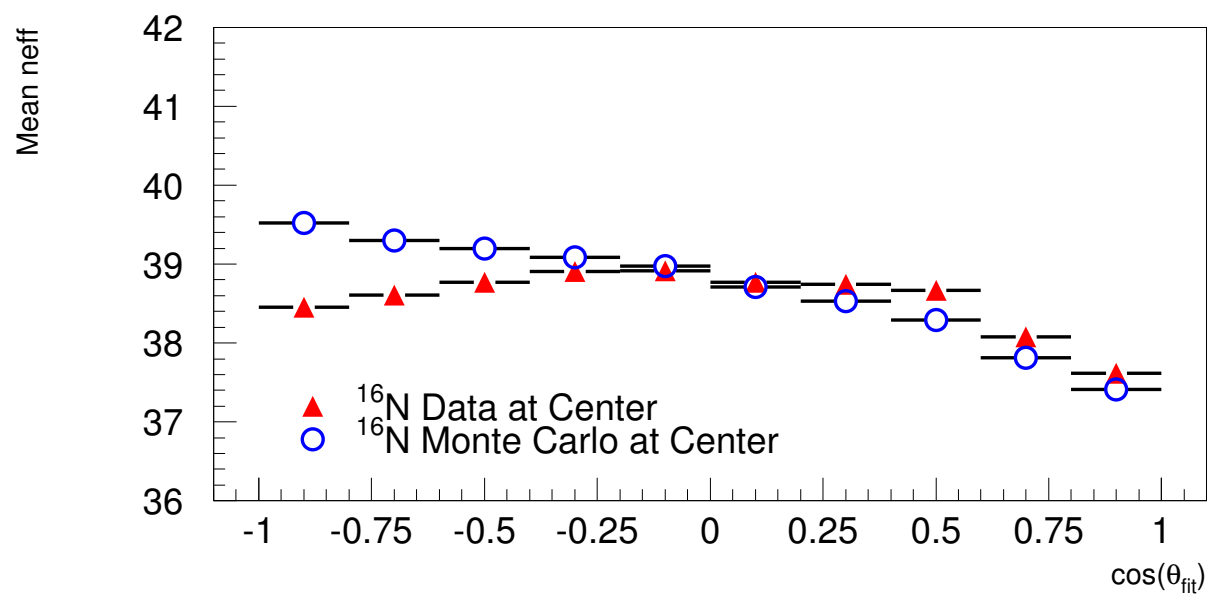

Figure 4.9: The mean $n_{e f f}$ as a function of the fit direction for many ${ }^{16} \mathrm{~N}$ runs at the center of the detector combined together. $\mathrm{A} \cos \left(\theta_{f i t}\right)$ value of +1 indicates that the reconstructed direction is upward.

A larger concern however is that the variation of the Monte Carlo simulation as a function of angle looks different than the variation of the data, especially around $\cos \left(\theta_{s}\right)=-$ 1. A similar effect can be seen in the ${ }^{252} \mathrm{Cf}$ source neutron data (though perhaps with a reduced magnitude since neutron events are less directional), as shown in Figure 4.10. This suggests that this effect might be related to an asymmetry in the detector response which is not modeled in the Monte Carlo simulations, rather than a failure to properly model the geometry of the ${ }^{16} \mathrm{~N}$ source.

The Monte Carlo simulation has been adjusted to match the data response by averaging over all angles. So, this is not an issue for the NC signal, where the discrepancy 
seen in the upward direction will balance out the discrepancy seen at the bottom of the detector. However, we might be concerned about the effect on a non-uniform distribution such as the CC and ES signal. For example, if all of the ES events pointed down, then there would be a large discrepancy between the data and MC. Again to evaluate the size of this discrepancy, ES and CC Monte Carlo simulation files with the appropriate run dates and times were examined. Averaging over all angles weighted by the expected angular distribution, the discrepancy between the data and MC was found to be $0.35 \%$ for the $\mathrm{CC}$ signal and $0.21 \%$ for the ES signal.

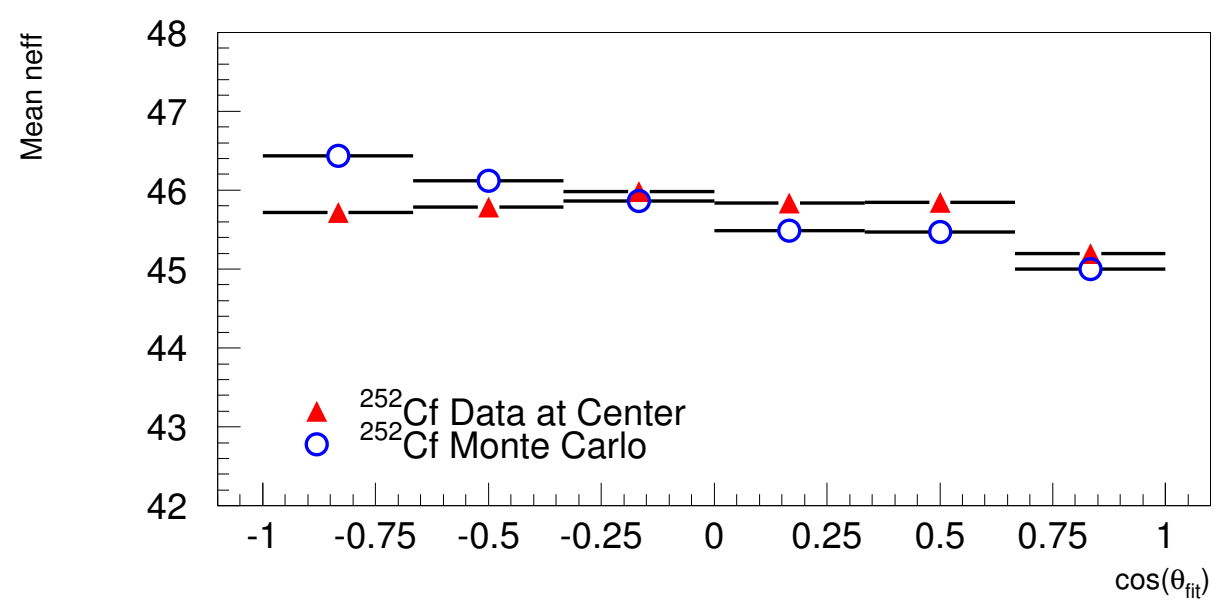

Figure 4.10: The mean $n_{\text {eff }}$ as a function of the fit direction for many ${ }^{252} \mathrm{Cf}$ runs at the center of the detector combined together. $A \cos \left(\theta_{\text {fit }}\right)$ value of +1 indicates that the direction is upward. 


\subsection{Conversion of $n_{e f f}$ to Energy}

So far we have described $n_{\text {eff }}$ and verified that the response of the data matches that of the Monte Carlo simulation. In this dissertation $n_{\text {eff }}$ will be the primary estimator of event energy. However, in order to extract meaningful quantities, we need to know how $n_{\text {eff }}$ translates into real energy. To do this, Monte Carlo simulated electrons were generated uniformly throughout the $\mathrm{D}_{2} \mathrm{O}$. Figure 4.11 shows the mean $n_{\text {eff }}$ for Monte Carlo simulated electrons of various energies. To obtain a relationship between $n_{\text {eff }}$ and visible energy (i.e. the electron kinetic energy), it has been fit to a parabola, yielding the following conversion:

$$
\text { Visible Energy }=0.71+0.1064 \times n_{e f f}+8.95 \times 10^{-5} \times n_{e f f}^{2}
$$

Note that according to this conversion, an electron must have at least $0.71 \mathrm{MeV}$ to be observed. The Cerenkov light production threshold for an electron is approximately 0.77 MeV. However the Čerenkov light output is non-linear below approximately 1.5 MeV (See for example Figure 19.2 in [87]). The visible energy here represents the electron kinetic energy. So, for a $\gamma$-ray this can be interpreted as the equivalent electron kinetic energy. Throughout this dissertation, this will be referred to as visible energy, and this is the energy that will be used to define energy thresholds. A $5.5 \mathrm{MeV}$ threshold is equivalent to 43.43 hits in $n_{e f f}$. The uncertainty on the parameterization in Equation 4.6 at $5.5 \mathrm{MeV}$ is 0.004 $\mathrm{MeV}$. 


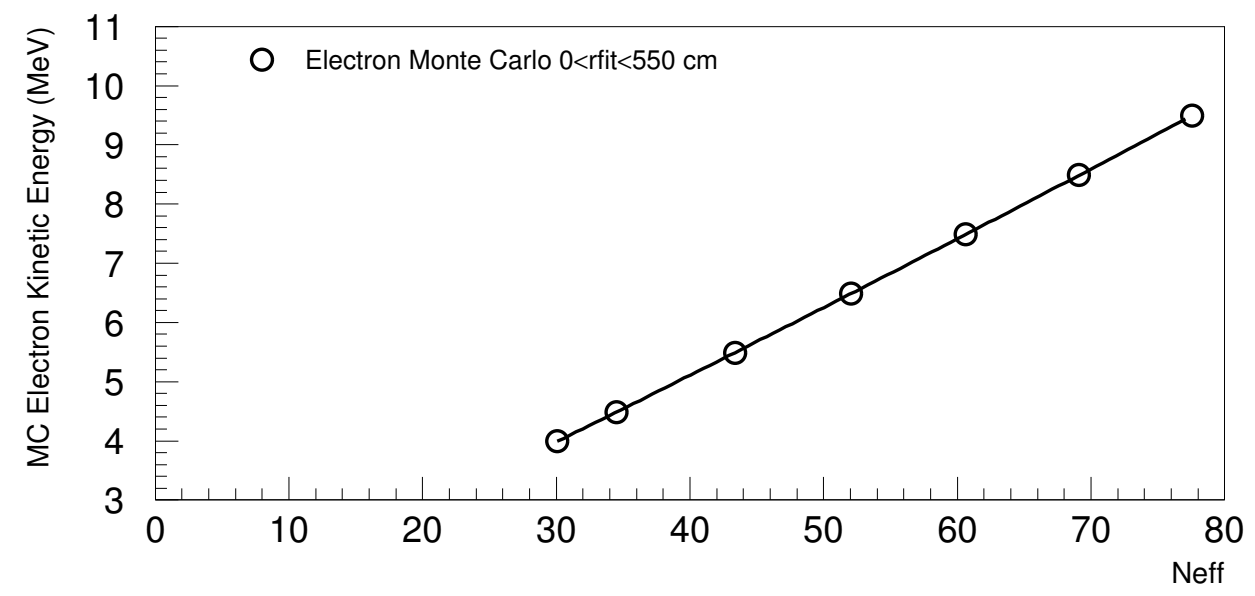

Figure 4.11: The kinetic energy for MC electrons vs the mean $n_{\text {eff }}$. It has been fit to a parabola.

\subsection{Energy Resolution}

So far, this chapter has focussed on the mean of the energy response. However, we must also evaluate the uncertainty on the width of the $n_{\text {eff }}$ distribution, which is effectively the energy resolution. The width of the $n_{\text {eff }}$ distribution as a function of the reconstructed radius is shown for ${ }^{16} \mathrm{~N}$ events in Figure 4.12. Though a discrepancy in the width can be seen in the $\mathrm{H}_{2} \mathrm{O}$, the data and $\mathrm{MC}$ agree to within 0.2 hits in the $\mathrm{D}_{2} \mathrm{O}$.

Figure 4.13 shows a similar plot for ${ }^{252} \mathrm{Cf}$ data and Monte Carlo simulations. Here the discrepancy is much larger, 0.35 hits. The reason for this discrepancy is unknown and will be treated as a systematic uncertainty. 


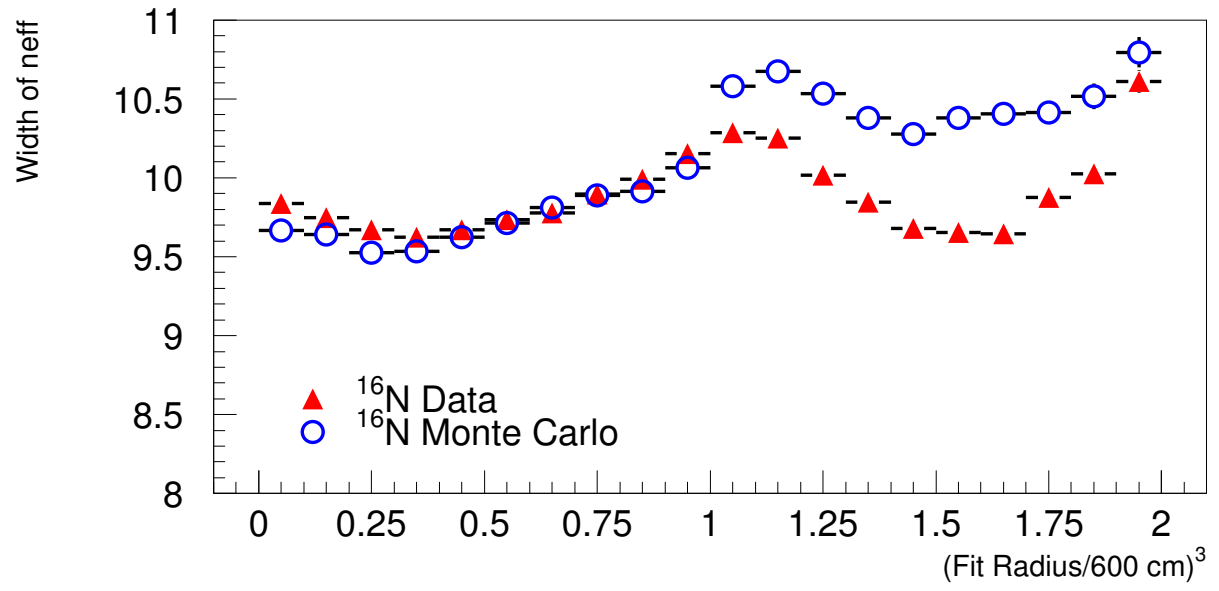

Figure 4.12: The width of $n_{\text {eff }}$ for ${ }^{16} \mathrm{~N}$ data and MC.

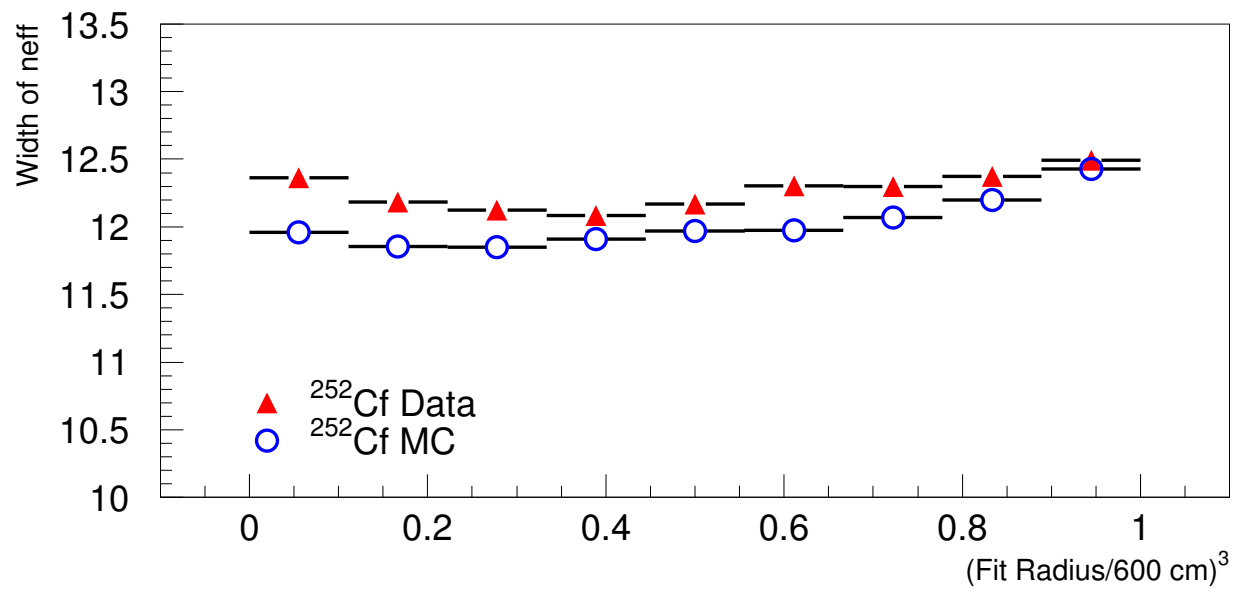

Figure 4.13: The width of $n_{\text {eff }}$ for ${ }^{252} \mathrm{Cf}$ data and MC. 


\subsection{Summary of Energy Uncertainty}

The primary uncertainties on the energy response are given in Table 4.1 for events within $550 \mathrm{~cm}$ of the center of the detector. For the ES and CC signals, the AmBe source data was used to obtain the uncertainty on the agreement between the data and MC. The ${ }^{16} \mathrm{~N}$ source was used for the other uncertainties on the $\mathrm{CC}$ and $\mathrm{ES}$ events. For the $\mathrm{NC}$ signal, ${ }^{252} \mathrm{Cf}$ neutron data was used wherever possible, but for example the time correction was based on the ${ }^{16} \mathrm{~N}$ source data due to its greater time coverage.

Adding together in quadrature all of the uncertainties on the energy at a threshold of $5.5 \mathrm{MeV}$, we obtain a combined uncertainty of $0.044 \mathrm{MeV}$, or $0.81 \%$. Though there is fairly good agreement on the energy resolution for the ${ }^{16} \mathrm{~N}$ data and Monte Carlo simulations, there is a discrepancy in the energy resolution seen in the ${ }^{252} \mathrm{Cf}$ data and Monte Carlo simulations. This will be treated as a systematic uncertainty in the determination of the number of neutrino events.

\begin{tabular}{c|c|c|c|}
\hline \hline Source of & \multicolumn{3}{|c|}{ Uncertainty (\%) } \\
\cline { 2 - 4 } Uncertainty & CC & ES & NC \\
\hline \hline Agreement Between Data and MC & 0.70 & 0.70 & 0.58 \\
\hline Radial Variation of Energy Scale & 0.21 & 0.21 & 0.51 \\
\hline Time Correction of Energy Scale & 0.06 & 0.06 & 0.06 \\
\hline Directional Variation & 0.004 & 0.06 & 0.0 \\
\hline Directional Diff Between Data and MC & 0.35 & 0.21 & 0.00 \\
\hline Uncertainty in $n_{e f f}$ to Tot. E scale & 0.06 & 0.06 & 0.06 \\
\hline \hline Total Uncertainty on Energy Scale & 0.81 & 0.77 & 0.77 \\
\hline \hline Uncertainty on Energy Resolution & 1.5 & 1.5 & 2.9 \\
\hline \hline
\end{tabular}

Table 4.1: Energy systematics at an energy threshold of $5.5 \mathrm{MeV}$ (visible energy). 


\section{Chapter 5}

\section{Event Isotropy}

\subsection{Using Light Isotropy to Distinguish Neutrino Event Types}

As was mentioned in Chapter 2, neutrinos are detected in SNO via three interactions: the Charged-Current (CC), Elastic-Scattering (ES), and Neutral-Current (NC) interactions. The CC and ES interactions both produce a single electron that is detected by its cone of Čerenkov light above some energy threshold. The NC interaction produces a free neutron. In the salted $\mathrm{D}_{2} \mathrm{O}$ of the $\mathrm{SNO}$ detector, neutrons will typically capture on ${ }^{35} \mathrm{Cl}$, yielding an excited state of ${ }^{36} \mathrm{Cl}$. Its de-excitation to the ground state produces a cascade of $\gamma$-rays with a total energy of $8.6 \mathrm{MeV}$. Over $97 \%$ of these transitions will produce two or more $\gamma$-rays . Details of the ${ }^{36} \mathrm{Cl}$ level scheme can be found in [88].

The multiple uncorrelated $\gamma$-rays give an additional handle on distinguishing the NC neutrino signal from the CC and ES signals. The light distribution from NC events is in general more isotropic than that of $\mathrm{CC}$ and ES events. Therefore, it is important to have a parameter that quantifies the isotropy of the light pattern for an event. 


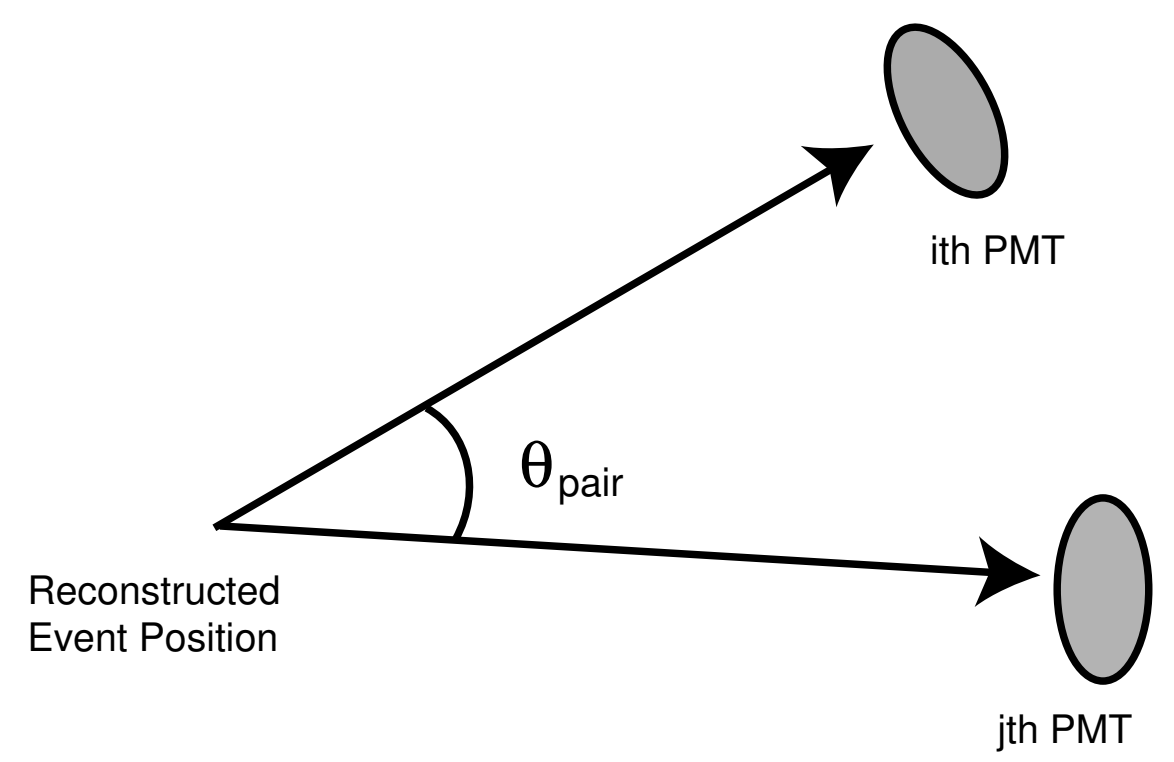

Figure 5.1: This schematic depicts $\theta_{\text {pair }}$, the angle between a pair of PMT hits relative to the reconstructed position. $\theta_{i j}$ is defined as the mean $\theta_{\text {pair }}$ for all possible pairings of prompt photo-multiplier tube hits.

\section{$5.2 \theta_{i j}$ - Measure of Isotropy}

The parameter which will be used in this dissertation to describe the event isotropy, $\theta_{i j}$, is the mean angle between pairs of PMTs. A schematic of the pair angle, $\theta_{\text {pair }}$, is shown for two photo-multiplier tubes in Figure 5.1. Note that it is defined relative to the reconstructed event vertex. $\theta_{i j}$ is the mean value of $\theta_{\text {pair }}$ (in radians), averaged over all possible pairs of PMTs. To exclude reflected light and other late hits, only prompt photomultiplier tube hits are used. (Using the definition given in Equation 3.1, prompt means hits with $\left|t_{\text {res }}\right|<9$ nsec.) A larger value of $\theta_{i j}$, signifying a larger average pair angle, indicates a more isotropic distribution of photo-multiplier tube hits.

Figure 5.2 shows the $\theta_{i j}$ distribution for $5 \mathrm{MeV}$ MC electrons. The distribution is not Gaussian in shape. However, the $\theta_{i j}$ spectrum for electrons can be well described by 


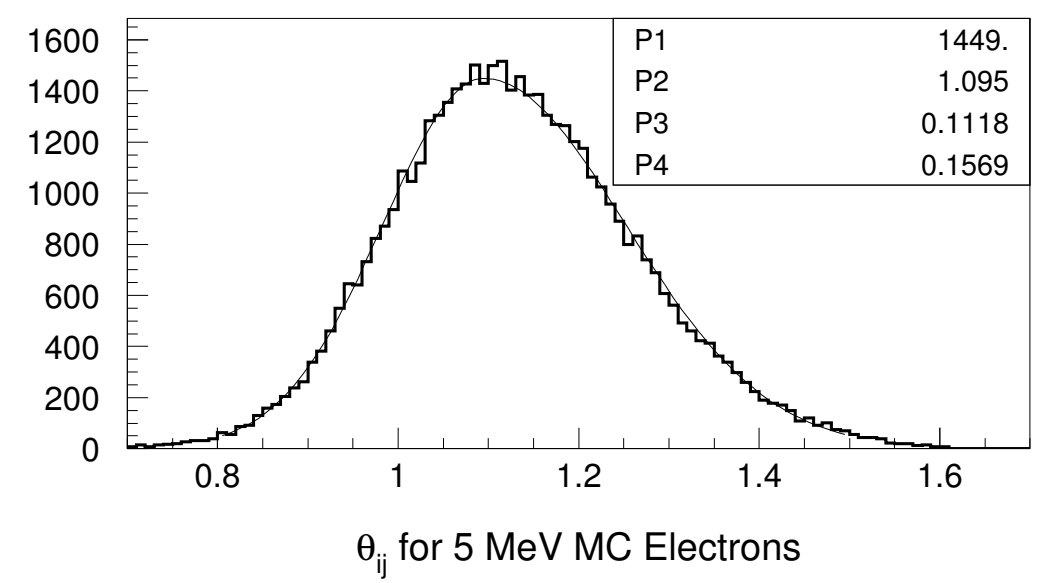

Figure 5.2: The $\theta_{i j}$ distribution for 4.5 MeV (kinetic energy) Monte Carlo simulated electrons. It has been fit to Equation 5.1.

two half-Gaussians, with different widths. In other words,

$$
N\left(\theta_{i j}\right)= \begin{cases}\left.p_{1} \times \exp \left(-\left(\theta_{i j}-p_{2}\right)^{2}\right) /\left(2 p_{3}^{2}\right)\right) & \text { for } \theta_{i j}<p_{2} \\ \left.p_{1} \times \exp \left(-\left(\theta_{i j}-p_{2}\right)^{2}\right) /\left(2 p_{4}^{2}\right)\right) & \text { for } \theta_{i j} \geq p_{2} .\end{cases}
$$

Figure 5.2 shows $5 \mathrm{MeV}$ Monte Carlo simulated electrons fit using Equation 5.1. Here the amplitude of the two half-gaussians $\left(p_{1}\right)$ has been forced to match so that $p_{2}$ represents the peak of the distribution. Throughout this chapter, I will refer to $p_{2}$ as the peak of $\theta_{i j}, p_{3}$ as the lower width, and $p_{4}$ as the upper width.

\subsection{Temporal Variation of $\theta_{i j}$}

It is important to verify that the behavior of $\theta_{i j}$ is not varying over time. In order to do this, the ${ }^{16} \mathrm{~N}$ source data was used since there are many ${ }^{16} \mathrm{~N}$ runs taken at the center of the detector over time. Here several ${ }^{16} \mathrm{~N}$ source runs were examined and their resulting 


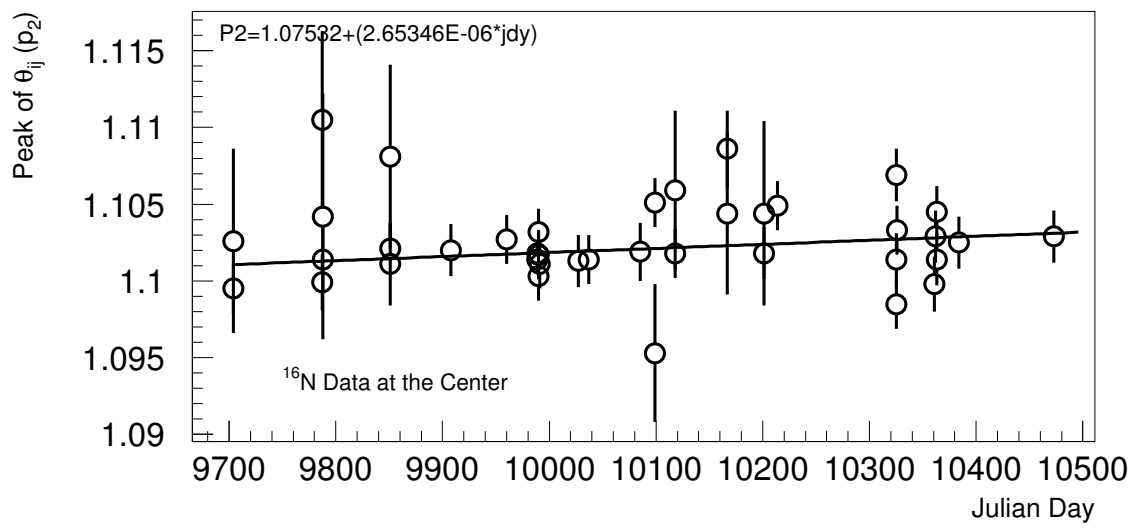

Figure 5.3: The fit values obtained for the peak of $\theta_{i j}$ ( $p_{2}$ in Equation 5.1) for ${ }^{16} \mathrm{~N}$ source runs at the center vs the Julian date of the source run.

$\theta_{i j}$ distributions were fit to the function described by Equation 5.1. The ${ }^{16} \mathrm{~N}$ events with the source at the center of the detector were required to have at least 20 hits, to reconstruct within $400 \mathrm{~cm}$ of the center of the detector, to have an observed beta tag, and to pass the instrumental background cuts which will be described in Chapter 7. Figures 5.3, 5.4, and 5.5, show plots of the $\theta_{i j}$ peak, lower and upper widths as a function of the Julian day.

From the resulting linear fits, it can be seen that over the 600 days of the salt phase, the variation over time on the peak of $\theta_{i j}$ is $0.09 \%$. The temporal variation on the upper width is $1.3 \%$, and the variation on the lower width is $0.3 \%$.

\subsection{Energy Dependence of $\theta_{i j}$}

We must also consider the variation of $\theta_{i j}$ with the energy of the event. Figure 5.6 shows the $\theta_{i j}$ distributions of MC electrons of various energies. The electrons have been 


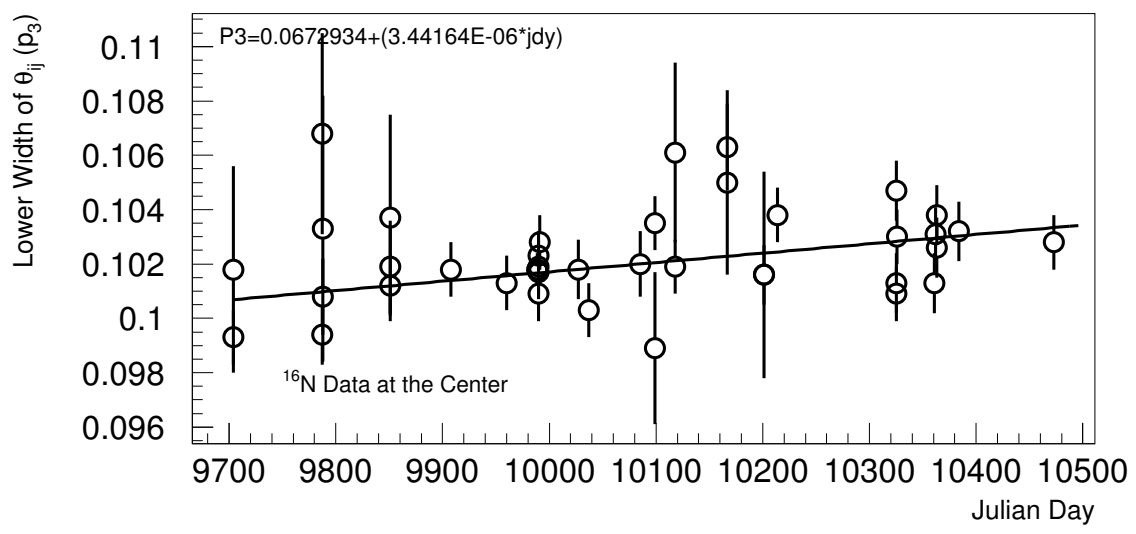

Figure 5.4: The fit values obtained for the lower width of $\theta_{i j}\left(p_{3}\right.$ in Equation 5.1) for ${ }^{16} \mathrm{~N}$ source runs at the center vs the Julian date of the source run.

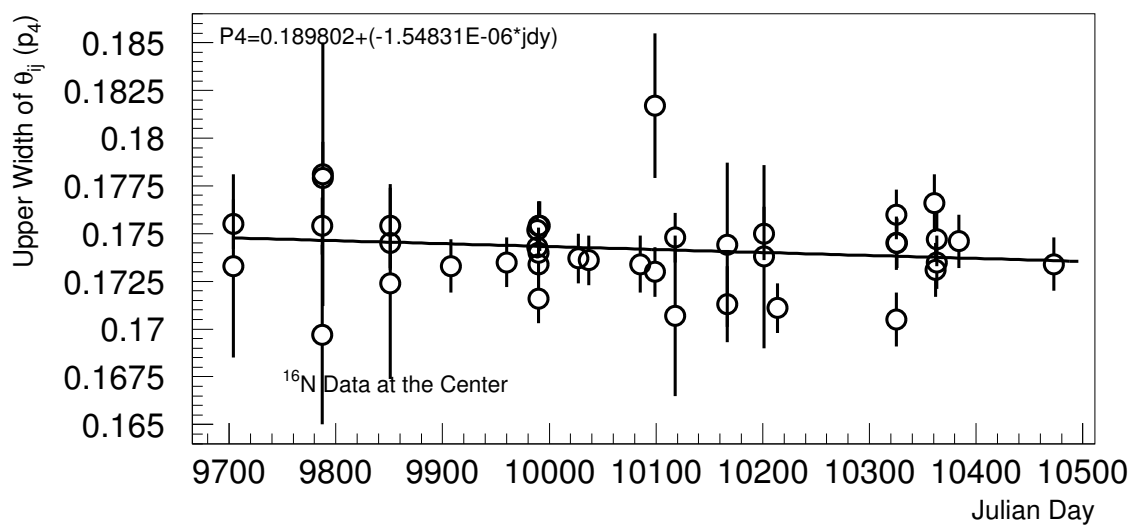

Figure 5.5: The fit values obtained for the upper width of $\theta_{i j}\left(p_{4}\right.$ in Equation 5.1) for ${ }^{16} \mathrm{~N}$ source runs at the center vs the Julian date of the source run. 


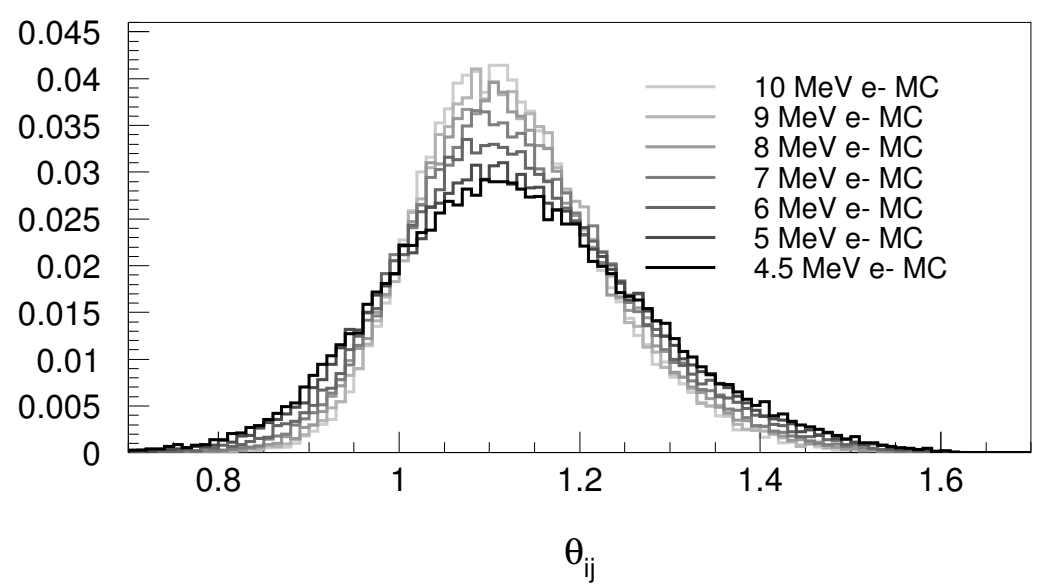

Figure 5.6: The $\theta_{i j}$ distributions for MC electrons at several different energies. The energies shown here are the total electron energy.

distributed uniformly throughout the heavy water. As the energy of the electrons increases, the $\theta_{i j}$ distribution becomes narrower. The increase in the number of tubes for the higher energy events leads to less statistical variation in $\theta_{i j}$. These distributions have also been fit to Equation 5.1. The resulting parameters have been plotted against the MC event energy in Figures 5.7, 5.8, and 5.9. The peak of the $\theta_{i j}$ distribution varies by approximately $1.3 \%$ over the energy region of interest for the neutrino analysis.

\subsection{Radial Dependence of $\theta_{i j}$}

We must also consider the variation of $\theta_{i j}$ with position. Again the ${ }^{16} \mathrm{~N}$ source was used. Figure 5.10 shows the fitted peak of the $\theta_{i j}$ distribution versus the reconstructed radial position for both ${ }^{16} \mathrm{~N}$ data and $\mathrm{MC}$ at a variety of source locations throughout the $\mathrm{D}_{2} \mathrm{O}$ and $\mathrm{H}_{2} \mathrm{O}$. Figures 5.11 and 5.12 show similar plots for the upper width and the 


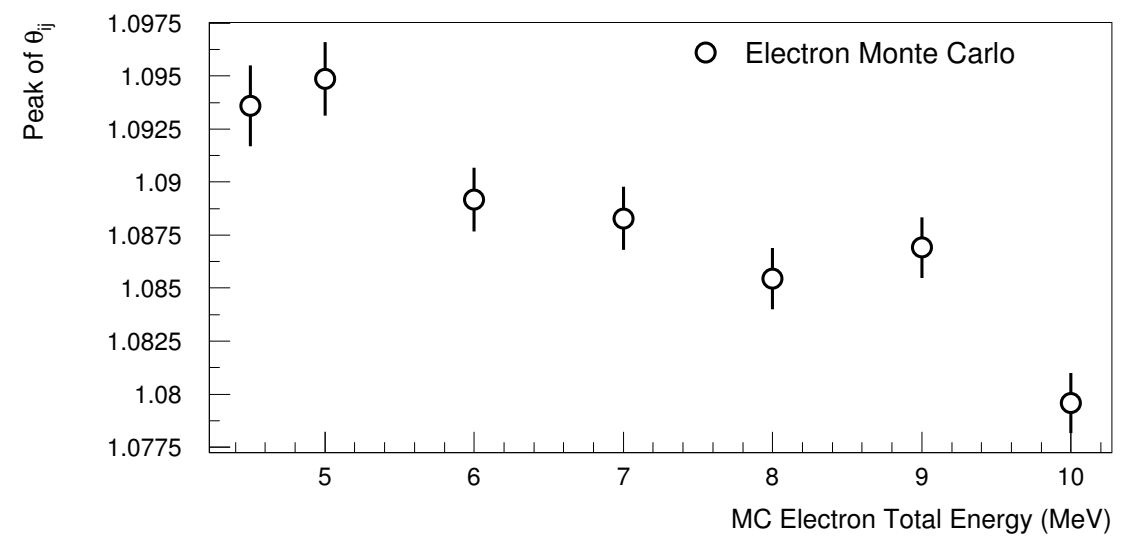

Figure 5.7: The fit value of the $\theta_{i j}$ peak for MC electrons of various energies.

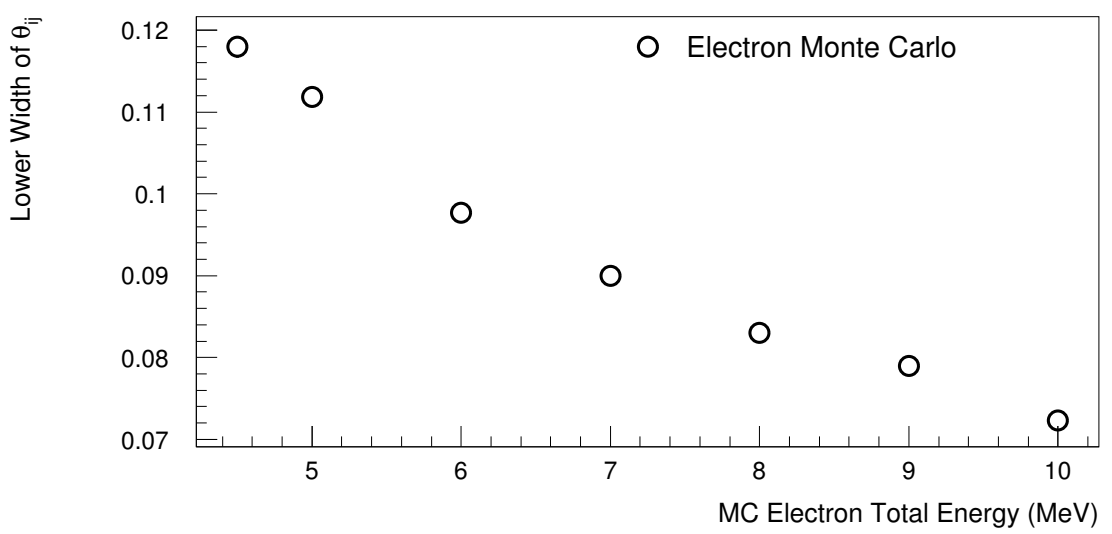

Figure 5.8: The fit value of the lower width of $\theta_{i j}$ for MC electrons of various energies. 


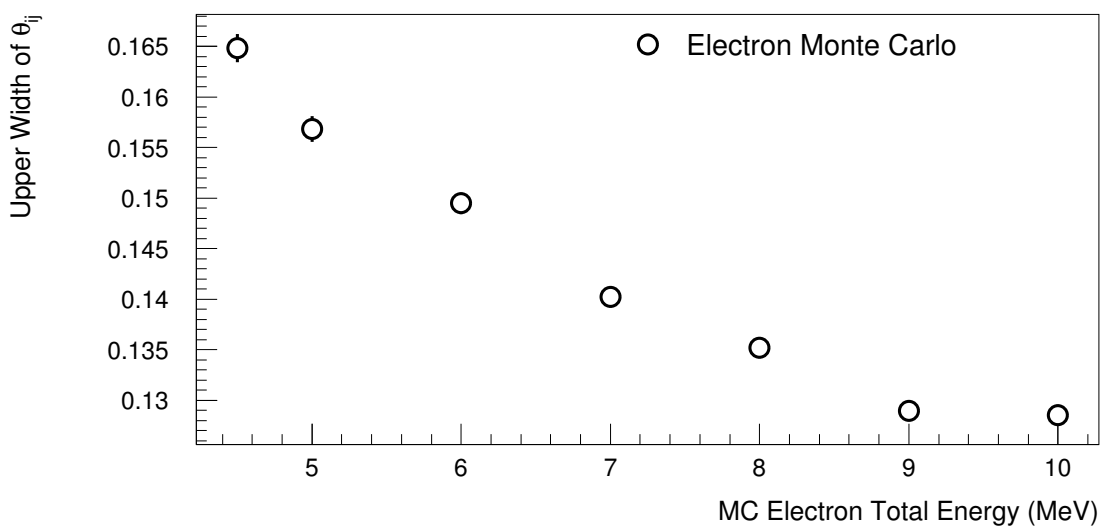

Figure 5.9: The fit value of the upper width of $\theta_{i j}$ for $\mathrm{MC}$ electrons of various energies.

lower width. Though a discrepancy is visible near the acrylic vessel (corresponding to a fit radius $/ 600 \mathrm{~cm}$ of 1$)$, within the fiducial volume $\left(\mathrm{r}<550 \mathrm{~cm}\right.$, or $\left.(\mathrm{r} / 600 \mathrm{~cm})^{3}<0.770\right)$ the agreement between the Monte Carlo simulations and the data appears quite good for all three parameters. Inside $550 \mathrm{~cm}$ the volume weighted difference between the data and Monte Carlo simulations is $0.54 \%$ for the peak, $1.4 \%$ for the lower width, and $1.0 \%$ for the upper width.

\section{$5.6 \theta_{i j}$ for neutrons}

It is also necessary to evaluate the performance of $\theta_{i j}$ for neutrons, using data from the ${ }^{252} \mathrm{Cf}$ source. As was the case with the studies of reconstruction and energy, a burst analysis has been applied to the source data. Only events in bursts of 3-10 events are shown here and the first event in a burst is not used, since there can be contamination 


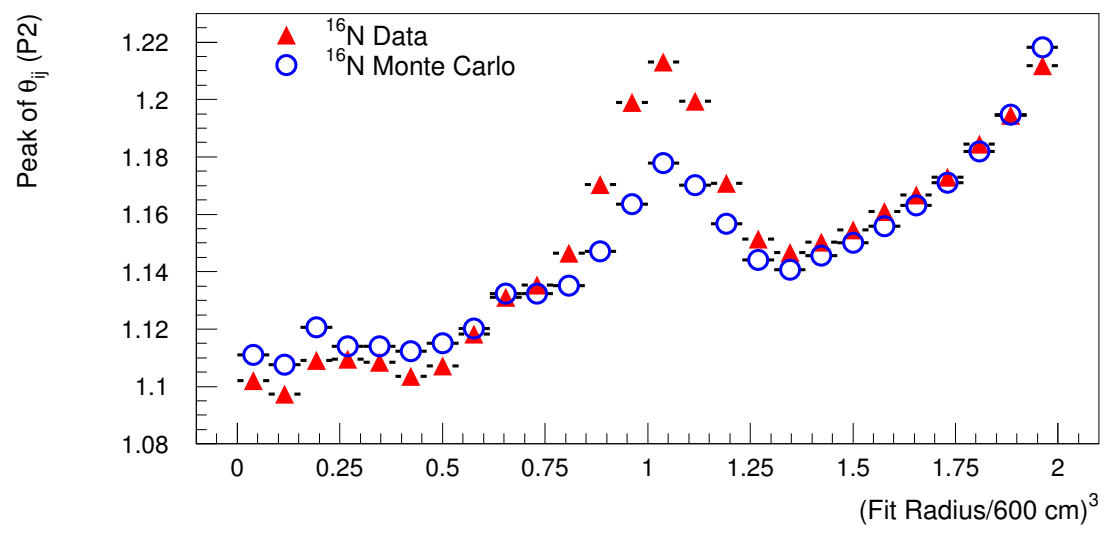

Figure 5.10: The fit values of the $\theta_{i j}$ peak plotted against the reconstructed event position for ${ }^{16} \mathrm{~N}$ source runs at a variety of locations.

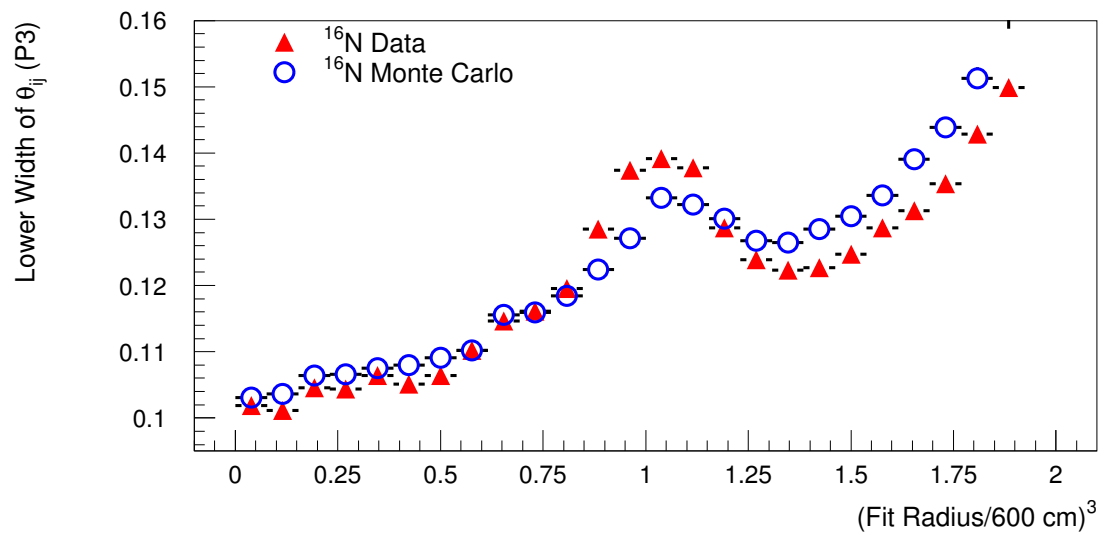

Figure 5.11: The fit values of the $\theta_{i j}$ lower width, $p_{3}$, plotted against the reconstructed event position for ${ }^{16} \mathrm{~N}$ source runs at a variety of locations. 


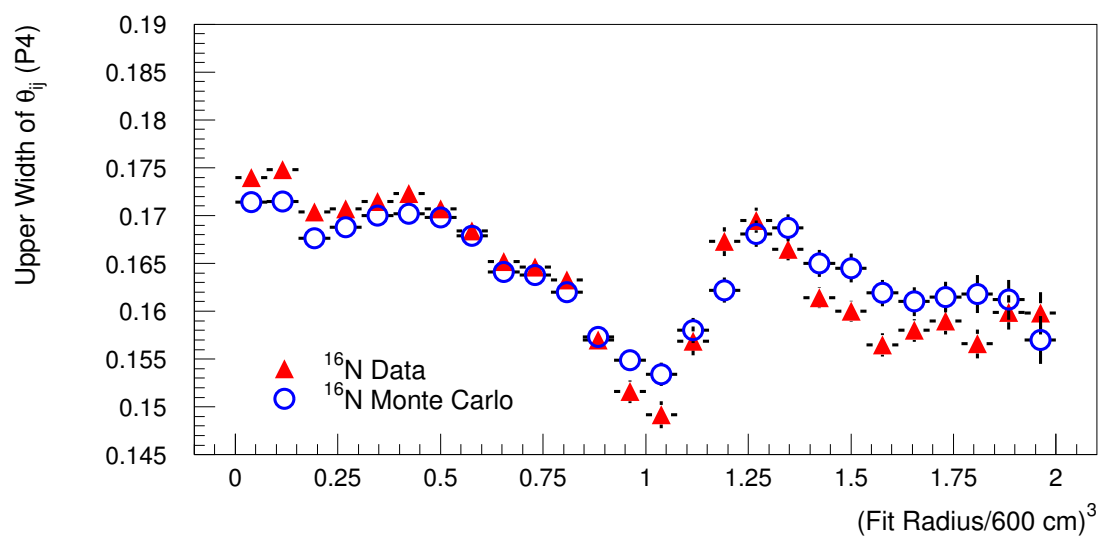

Figure 5.12: The fit values of the $\theta_{i j}$ upper width, $p_{4}$, plotted against the reconstructed event position for ${ }^{16} \mathrm{~N}$ source runs at a variety of locations.

from prompt fission $\gamma$-rays. Figure 5.13 shows the $\theta_{i j}$ distribution for neutrons from a ${ }^{252} \mathrm{Cf}$ source run located near the center of the detector. It has been fit to Equation 5.1. The fit is not quite as good as the fit to the ${ }^{16} \mathrm{~N}$ source data, especially for large values of $\theta_{i j}$. However, we can still use this parameterization to evaluate the differences between the data and the MC $\theta_{i j}$ shapes.

Figure 5.14 shows the fitted peak of the the $\theta_{i j}$ distribution versus the reconstructed radial position for ${ }^{252} \mathrm{Cf}$ data and MC. Several ${ }^{252} \mathrm{Cf}$ runs throughout the $\mathrm{D}_{2} \mathrm{O}$ have been summed together. Figures 5.15 and 5.16 show similar plots for the upper width and the lower width. As was the case for the ${ }^{16} \mathrm{~N}$ source, within the fiducial volume $(\mathrm{r}<550$ $\mathrm{cm}$, or $\left.(\mathrm{r} / 600 \mathrm{~cm})^{3}<0.770\right)$, the agreement between the Monte Carlo simulations and the data appears quite good for all three parameters. Inside $550 \mathrm{~cm}$ the volume weighted difference between the data and Monte Carlo simulations is $0.1 \%$ for the peak, $1.1 \%$ for the 


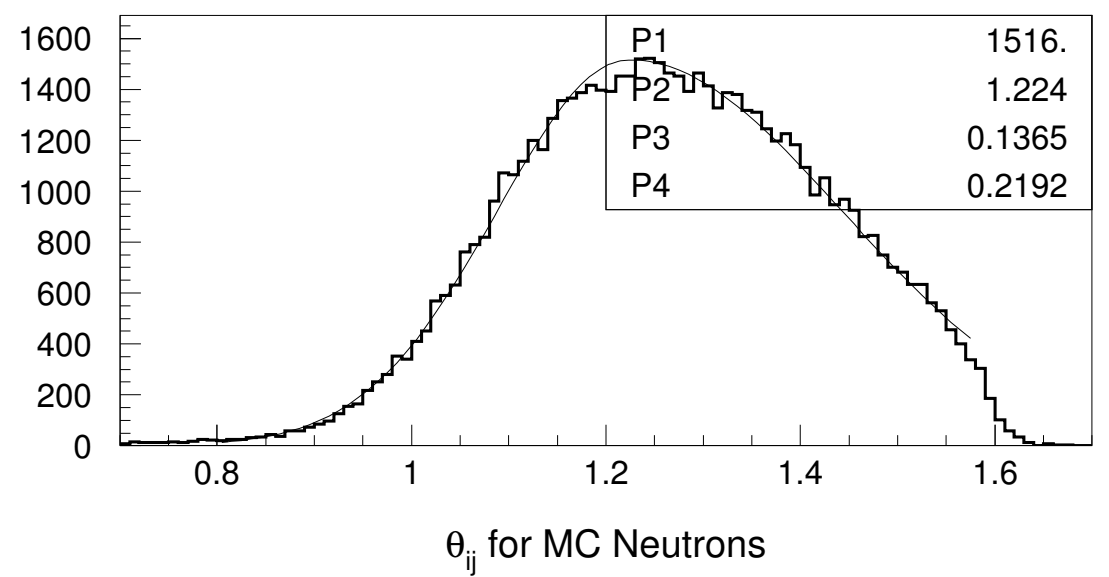

Figure 5.13: The $\theta_{i j}$ distribution for Monte Carlo simulated neutrons. It has been fit to Equation 5.1.

lower width, and $1.0 \%$ for the upper width.

\section{7 $\theta_{i j}$ for 4.4 MeV Gammas from AmBe source}

As a final cross check, we can also look at the $4.4 \mathrm{MeV} \gamma$-rays from the AmBe source. Using the procedure described in Chapter 4, we can obtain a clean sample of 4.4 $\mathrm{MeV} \gamma$-rays by looking for neutrons and subtracting the events just after a neutron from the events just prior to a neutron. Figure 5.17 shows a volume-weighted $\theta_{i j}$ distribution of 4.4 $\mathrm{MeV} \gamma$-ray events from data and MC. When the distributions are fit to Equation 5.1, the peak for the data is $1.156 \pm 0.012$ (stat.) and the peak of the MC is $1.141 \pm 0.004$ (stat.). The lower width of the data is $0.1463 \pm 0.0084$ and the peak of the MC is $0.1391 \pm 0.0026$. The upper width is $0.1903 \pm 0.0100$ and the peak of the MC is $0.1904 \pm 0.0032$. Though the $\mathrm{MC}$ and data peaks differ by $1.3 \%$, the statistics are poor and this discrepancy is only $1.25 \sigma$. 


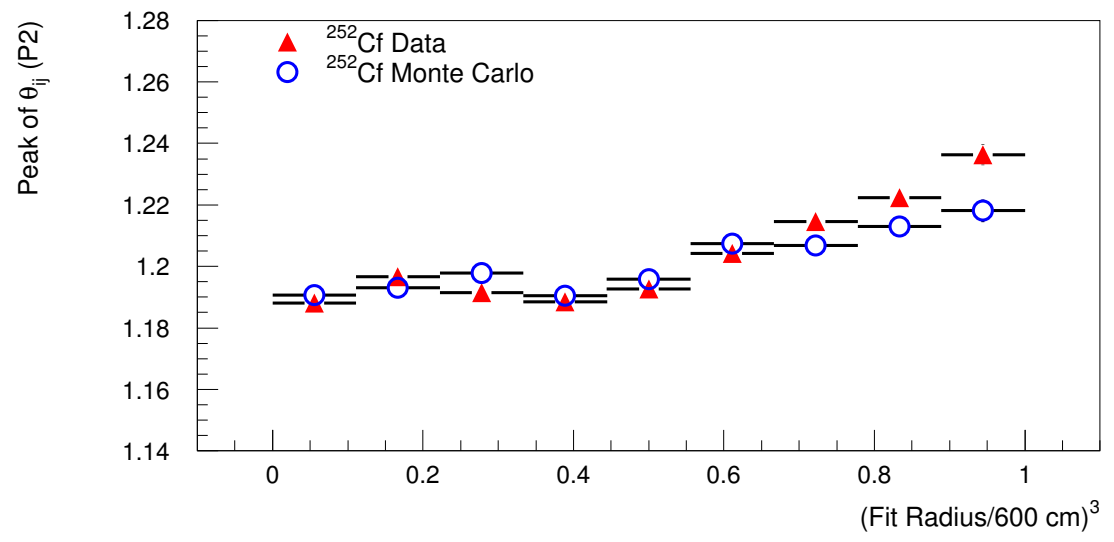

Figure 5.14: The fit value of the $\theta_{i j}$ peak plotted against the reconstructed event position for ${ }^{252} \mathrm{Cf}$ source runs at a variety of locations.

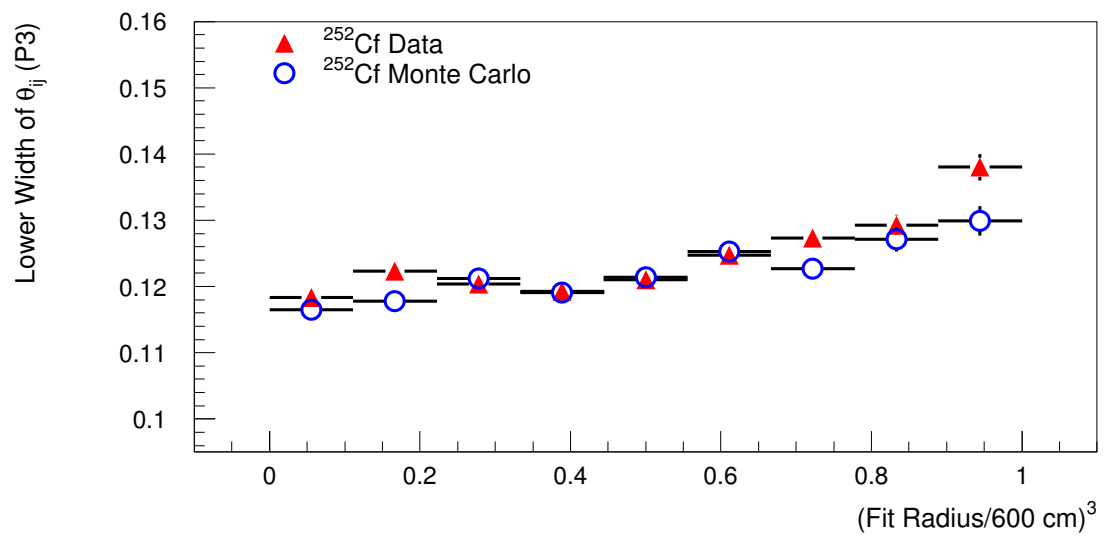

Figure 5.15: The fit value of the $\theta_{i j}$ lower width, $p_{3}$, plotted against the reconstructed event position for ${ }^{252} \mathrm{Cf}$ source runs at a variety of locations. 


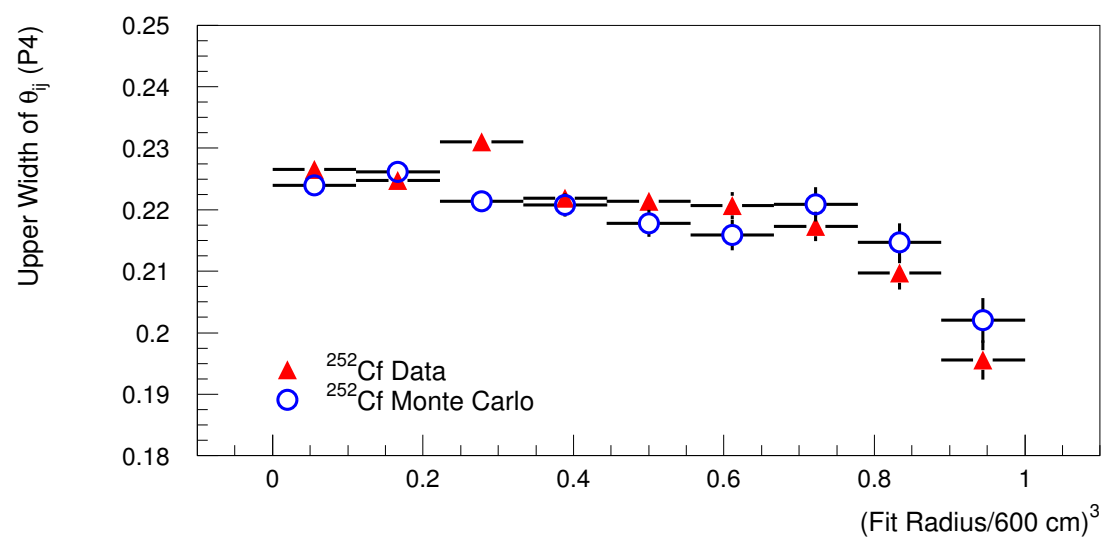

Figure 5.16: The fit value of the $\theta_{i j}$ upper width, $p_{4}$, plotted against the reconstructed event position for ${ }^{252} \mathrm{Cf}$ source runs at a variety of locations.

\subsection{Summary of Uncertainties on $\theta_{i j}$}

Table 5.1 summarizes the uncertainties on the various parameters describing the $\theta_{i j}$ shape. The temporal variation of the parameters was measured with the ${ }^{16} \mathrm{~N}$ source since there are far fewer central ${ }^{252} \mathrm{Cf}$ runs. It is assumed here that the neutrons will show the same temporal variation. The primary uncertainty on the $\theta_{i j}$ spectrum is due to the differences seen between the data and MC. To obtain the total uncertainty, the individual uncertainties have been added in quadrature. 


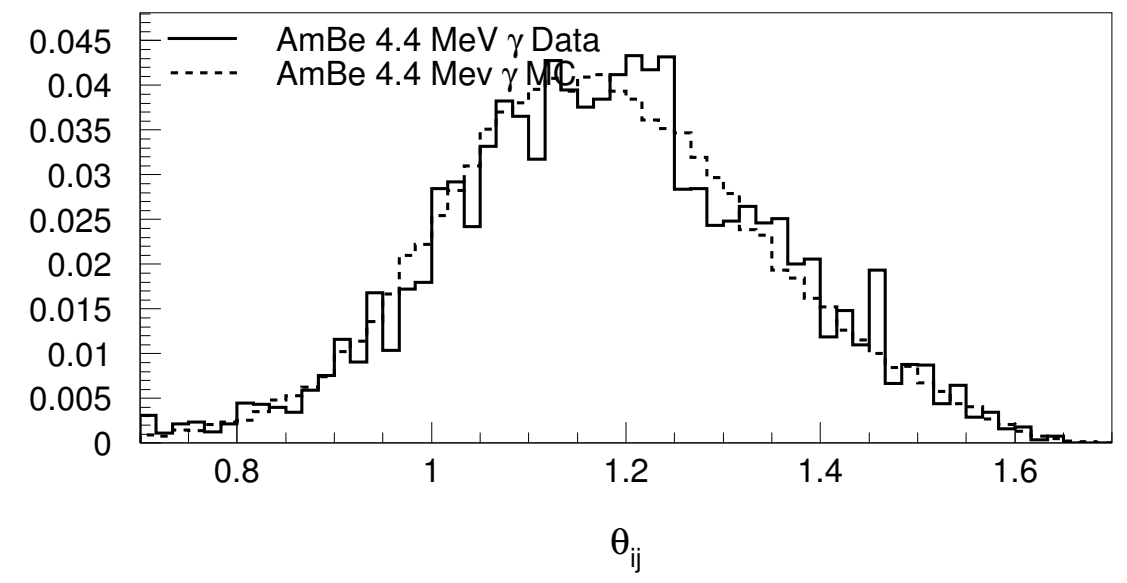

Figure 5.17: The volume-weighted $\theta_{i j}$ distribution for data and Monte Carlo simulated 4.4 $\mathrm{MeV} \gamma$-ray events from the AmBe source.

\begin{tabular}{|c|c||c|c|}
\hline \hline Parameter & Source & $\begin{array}{c}\text { Electron } \\
\text { Uncert }(\%)\end{array}$ & $\begin{array}{c}\text { Neutron } \\
\text { Uncert }(\%)\end{array}$ \\
\hline \hline$p_{2}$ & Diff Between Data and MC & 0.6 & 0.1 \\
\hline$p_{2}$ & Time Variation & 0.09 & 0.09 \\
\hline$p_{2}$ & Total Uncertainty & 0.6 & 0.13 \\
\hline \hline$p_{3}$ & Diff Between Data and MC & 1.4 & 1.1 \\
\hline$p_{3}$ & Time Variation & 1.3 & 1.3 \\
\hline$p_{3}$ & Total Uncertainty & 1.9 & 1.7 \\
\hline \hline$p_{4}$ & Diff Between Data and MC & 1.0 & 1.0 \\
\hline$p_{4}$ & Time Variation & 0.3 & 0.3 \\
\hline$p_{4}$ & Total Uncertainty & 1.0 & 1.0 \\
\hline \hline
\end{tabular}

Table 5.1: Systematic Uncertainties on Isotropy Parameter $\theta_{i j}$ 


\section{Chapter 6}

\section{Neutron Detection Efficiency}

\subsection{Neutron Capture}

In order to determine the neutrino flux via the neutral-current interaction, it is important to understand the neutron detection process in SNO. When a free neutron is produced, by the NC interaction or another means, it will rapidly thermalize and then walk randomly around the detector before capturing on a nucleus and producing $\gamma$-rays. As a result of the thermalization process, any information about the initial neutrino energy and direction is lost.

In the second phase of the $\mathrm{SNO}$ experiment, $\mathrm{NaCl}$ was added to the $\mathrm{D}_{2} \mathrm{O}$ to produce a $0.2 \%$ salt solution. Since ${ }^{35} \mathrm{Cl}$ has a $(n, \gamma)$ cross section which is over 80,000 times larger than that of ${ }^{2} \mathrm{H}$, most of the neutrons produced near the center of the detector will capture on ${ }^{35} \mathrm{Cl}$, which is $75.8 \%$ of natural $\mathrm{Cl}[60]$. Neutron capture on ${ }^{35} \mathrm{Cl}$ results in a cascade of $\gamma$-rays with a total energy of $8.6 \mathrm{MeV}$. Based on a MC simulation, a neutron will random walk an average of $70 \mathrm{~cm}$ in the salted $\mathrm{D}_{2} \mathrm{O}$ before capturing. A neutron at the center of the 
detector will randomly walk for approximately $5.5 \mathrm{msec}$ and neutrons distributed uniformly throughout the $\mathrm{D}_{2} \mathrm{O}$ will walk for an average time of approximately $5.1 \mathrm{msec}$.

If a neutron reaches the acrylic vessel or the $\mathrm{H}_{2} \mathrm{O}$, then there is a high probability that it will capture on ${ }^{1} \mathrm{H}$, which has a neutron capture cross section that is about $1 \%$ of the $\mathrm{Cl}$ capture cross section [60] but is present in a greater concentration. When a neutron captures on ${ }^{1} \mathrm{H}$, a $2.2 \mathrm{MeV} \gamma$-ray is emitted, which is below the detection threshold for the experiment. So, these neutrons are essentially lost. For this reason, we expect that the neutron detection efficiency will decrease at large radii.

In order to determine the flux of solar neutrinos via the neutral-current interaction, we must understand the total neutron detection efficiency integrated over the entire volume of interest and the competition between capture on $\mathrm{Cl}, \mathrm{D}$, and $\mathrm{H}$.

\subsection{The AmBe Source}

To evaluate the neutron detection efficiency inside a particular fiducial volume and above an energy threshold, we can use the Monte Carlo simulation. However, we should test the performance of the Monte Carlo simulations against calibration data. The primary source used in SNO for the calibration of the neutron detection efficiency is the

${ }^{252} \mathrm{Cf}$ source. However, this dissertation will present an alternate calibration technique using the Americium-Beryllium (AmBe) source. Though the ${ }^{252} \mathrm{Cf}$ source has been deployed in a large number of positions for a longer duration of time, the AmBe source has the advantage of being a tagged neutron source with only a single neutron emitted in each decay.

In this source, ${ }^{241} \mathrm{Am}$ emits $\alpha$-rays, which strike a ${ }^{9} \mathrm{Be}$ target, producing neutrons 


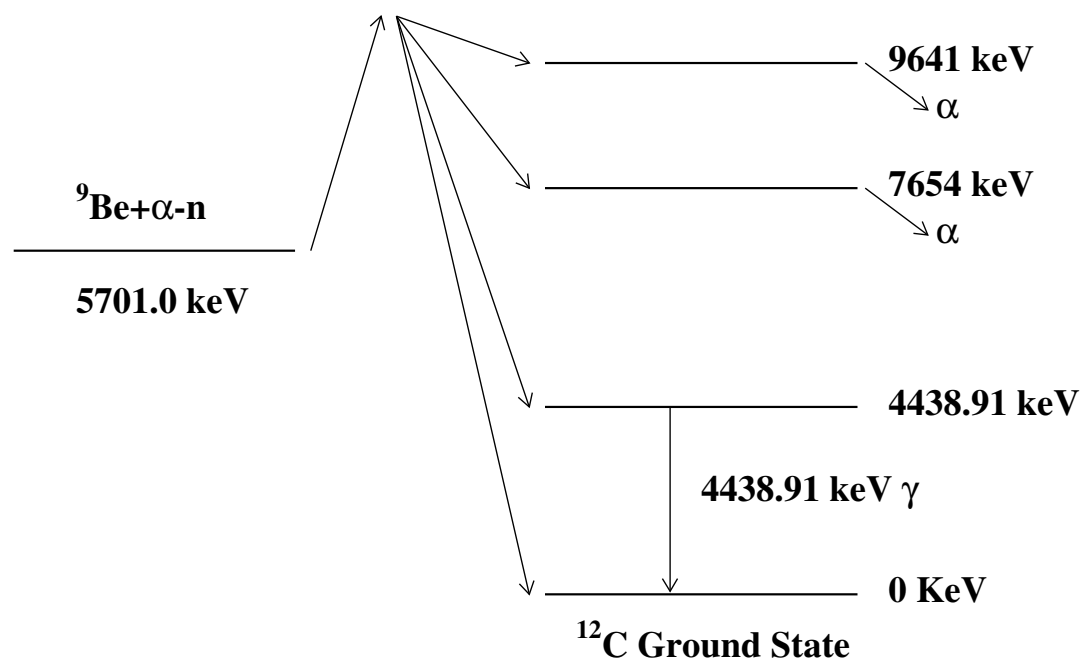

Figure 6.1: This drawing, based on one in [63], depicts the levels involved in the ${ }^{9} \mathrm{Be}+\alpha-\mathrm{n}$ reaction.

via the ${ }^{9} \mathrm{Be}(\alpha, n){ }^{12} \mathrm{C}$ reaction [62] (Q-value of $5.7 \mathrm{MeV}$ ). Figure 6.1 depicts the populated levels in ${ }^{12} \mathrm{C}$. Since the alphas from ${ }^{241} \mathrm{Am}$ have an energy up to $5.6 \mathrm{MeV}$ [60], there are three possible excited states of ${ }^{12} \mathrm{C}$ which can be populated in addition to the ground state. The two most energetic states (9641 keV and $7654 \mathrm{keV}$ ) almost always decay by $\alpha$ emission, and only emit a $\gamma$-ray in less than $0.000001 \%$ and $0.05 \%$ of all decays, respectively [63]. However, the $4438 \mathrm{keV}$ state will always emit a $\gamma$-ray. The exact ratio of $4.439 \mathrm{MeV} \gamma$-rays to neutrons depends on the initial spectrum of the $\alpha$ s. For the $\alpha \mathrm{s}$ from ${ }^{241} \mathrm{Am}$, it has been measured that $59.1 \pm 1.5$ percent of the reactions yield a $4.439 \mathrm{MeV} \gamma$-ray [63]. Since the neutrons will take time to thermalize and capture, the $\gamma$-ray will usually be detected before the neutron. The decay rate of the source was measured to be a few Hz. 
We can express the number of observed coincidences as

$$
N_{\text {coinc }}=N_{\text {neutron }} \times \frac{N_{\gamma}}{N_{\text {neutron }}} \times \epsilon_{n} \times \epsilon_{\gamma}
$$

where $\epsilon_{n}$ is the efficiency for detecting neutrons, $\epsilon_{\gamma}$ is the efficiency for detecting the 4.4

$\mathrm{MeV} \gamma$-rays, $N_{\gamma}$ is the number of $\gamma$-rays emitted by the source, and $N_{\text {neutron }}$ is the number of neutrons emitted by the source. This can be rearranged as

$$
\epsilon_{n}=\frac{N_{\text {coinc }}}{N_{\gamma} \times \epsilon_{\gamma}}
$$

Here the numerator is the number of observed coincidences and the denominator is the number of observed $\gamma$-rays. So, this gives a method to determine the neutron detection efficiency for a given source run.

\subsection{Determining the Number of $\gamma$-rays}

To determine the number of $\gamma$-rays in a given source run (the denominator in Equation 6.2), we can look at the energy distribution for the events in the run. We expect to see contributions from $\gamma$-rays and neutrons, in addition to the random backgrounds and neutrons we might expect with no source deployed. For the $\gamma$-ray energy response, we can use the Monte Carlo simulation. However, as was observed in Chapter 4 the energy scale has an uncertainty of $\pm 0.8 \%$. This is a systematic uncertainty which must be taken into account when using the MC for the $\gamma$-ray shape.

For the neutrons, we can use the AmBe source data to provide us with a neutron spectrum. To do this, we can look for isolated event pairs, presumably $\gamma$-n coincidences. We define a pair as having two and only two events within $30 \mathrm{msec}$ of one another, and no 
other events within 60 msec before or after the first event. Figure 6.2 shows a plot of $n_{\text {eff }}$ for the second event in a pair, which should be a neutron. ${ }^{1}$ Also shown in Figure 6.2 is the expected shape for neutrons from Monte Carlo simulations. The shape seen in the second event is in very good agreement with that expected for neutrons.

We also need to understand the shape of the background without a source. To do this, several neutrino runs taken just prior to the AmBe calibrations were combined together and scaled to reflect the contribution expected for the duration of the particular AmBe run.

With the $n_{\text {eff }}$ spectrum, the number of observed 4.4 MeV $\gamma$-rays in the AmBe run can be determined. Figure 6.3 shows the $n_{\text {eff }}$ spectrum for an AmBe run at the center. Only events with a reconstructed position within $725 \mathrm{~cm}$ of the center of the detector and $n_{\text {eff }}$ between 24 and 100 hits have been used. A fit has been performed to determine the number of neutrons and $\gamma$-rays present in the source run. This gives the number of observed $\gamma$-rays, which is the denominator in Equation 6.2.

\subsection{Determining the Number of Coincidences}

Now we must determine the total number of coincidences observed in the dataset.

To do this we can look at the distribution of time to the next event. In principle, we expect to see an exponential with a 5 msec time constant, corresponding to the time between a $\gamma$-ray and its associated neutron. For neutrons, we expect that the time to the next event should follow an exponential with a slope of a few $\mathrm{Hz}$, corresponding to the time between

\footnotetext{
${ }^{1} n_{e f f}$ is defined in Chapter 4.
} 


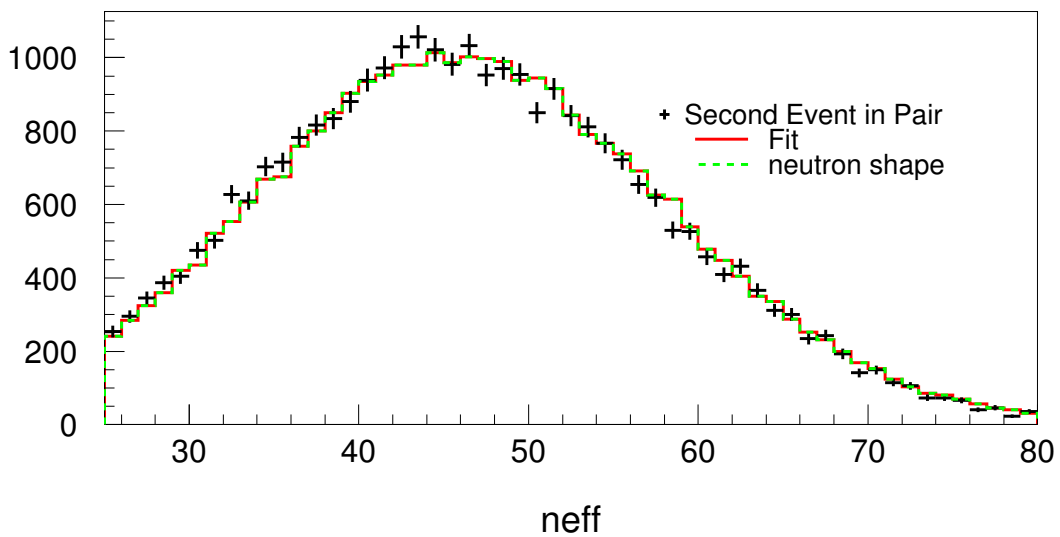

Figure 6.2: The $n_{\text {eff }}$ spectrum for the second event in a clean pair from an AmBe source run near the center. Also shown here is the expected MC neutron shape.

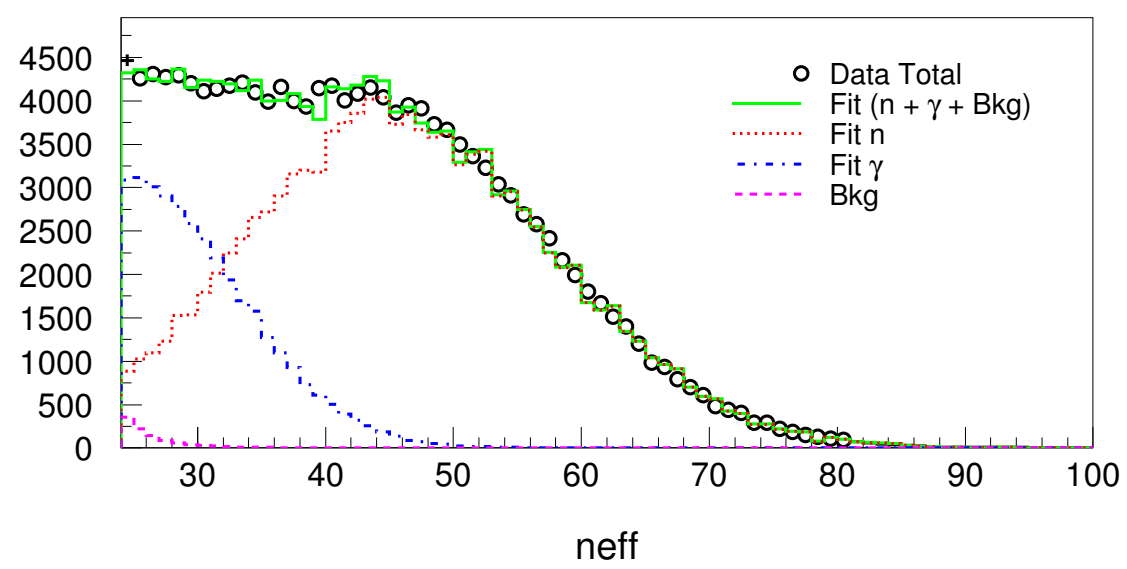

Figure 6.3: The $n_{\text {eff }}$ spectrum for all events with $R_{f i t}<725 \mathrm{~cm}$ for an AmBe source run at the center. It has been fit to determine the number of neutron and $\gamma$-ray events present. 


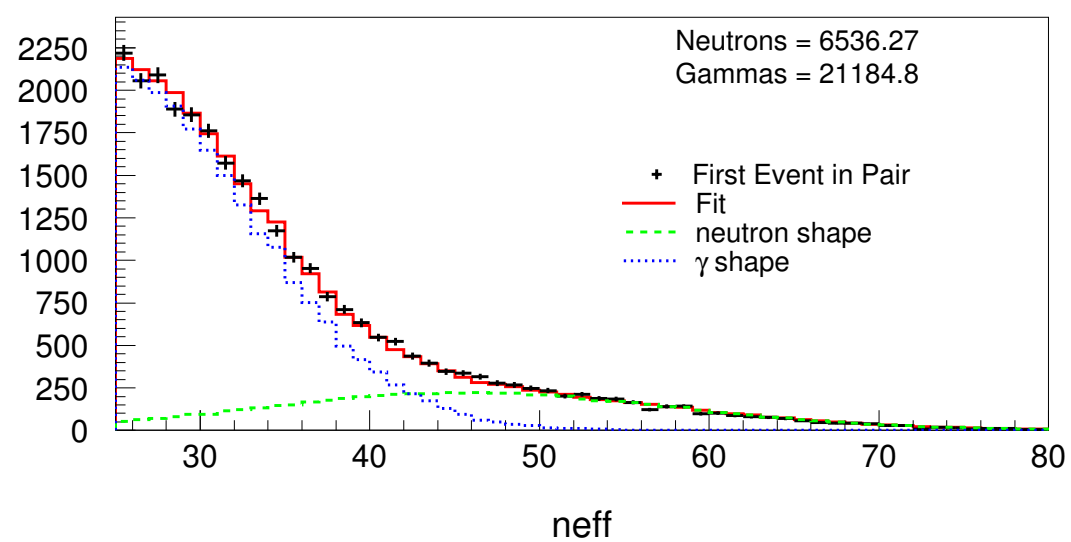

Figure 6.4: The $n_{\text {eff }}$ spectrum for the first event in a clean pair from an AmBe source run near the center. It has been fit to determine the number of neutron and $\gamma$-ray events.

subsequent AmBe source interactions.

To test this, we can look for neutrons by requiring them to have at least 50 hits and to reconstruct within $725 \mathrm{~cm}$ of the center of the detector. Figure 6.5 shows a plot of the time to the next event and the time to the previous event for "neutrons". The time to the previous event has the expected shape, with the $5 \mathrm{msec}$ component expected from the $\gamma$-ray coincidences and a long exponential corresponding to the time to a previous decay from the source. However, if we look at the time to the next event, it is not merely the exponential constant of $200-500$ msec that we would expect from the source decay rate. There is also a 5 msec component from the neutron capture time. This would suggest that some of the neutrons might have neutron afterwards. Further evidence of this is seen in Figures 6.4 and 6.2, which show the $n_{\text {eff }}$ for the first and second events in a pair. While the second events are consistent with the expected shape for neutrons, the first event appears to consist of both $\gamma$-rays and neutrons. For the run at the center shown in Figures 6.4 


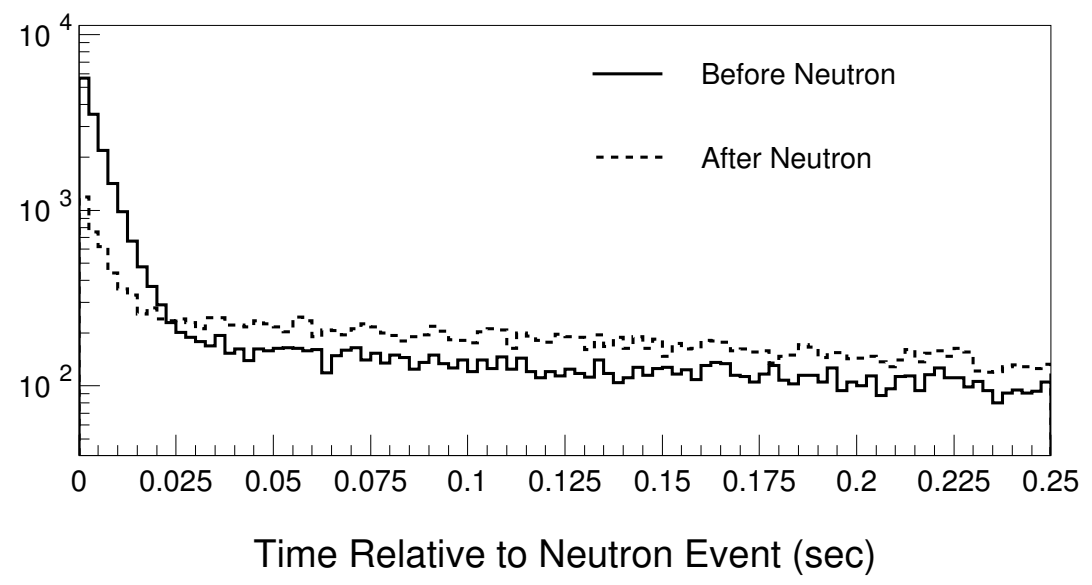

Figure 6.5: The time to the next event just before and just after a neutron. Here a neutron is defined as having more than 50 hits and a reconstructed position within $725 \mathrm{~cm}$ of the center of the detector. Before a neutron we expect to see a $5 \mathrm{msec}$ signal due to the $\gamma \mathrm{n}$ pairs coming from the source. However, a 5 msec component is also visible after a neutron, suggesting that some neutrons are correlated in time with other neutrons.

and 6.2 , roughly $24 \%$ of the first events in a pair appear to be neutrons. So, it appears that there are a significant number of neutron-neutron pairs in the data.

We might expect to see some neutron-neutron pairs due to the photodisintegration of deuterium by the $4.4 \mathrm{MeV} \gamma$-rays. A MC simulation reveals that $0.6 \%$ of $4.4 \mathrm{MeV}$ $\gamma$-rays will photodisintegrate deuterium to produce a neutron. Assuming that the detection efficiency above $n_{\text {eff }}=24$ is roughly $50 \%$ for $4.4 \mathrm{MeV}$-rays and roughly $95 \%$ for neutrons produced at the center of the detector, we can estimate that of the observed pairs, approximately $1 \%$ could be an n-n pair created by photodisintegration. The larger source of $n-n$ pairs is the $(n, 2 n)$ reaction on deuterium. This reaction has a threshold of $2.2 \mathrm{MeV}$. For the neutrons produced by the AmBe source, the Q-value for the reaction to the ground state of ${ }^{12} \mathrm{C}$ is $5.7 \mathrm{MeV}$. So, in the decays where a $4.4 \mathrm{MeV} \gamma$-ray is not emitted and the 
${ }^{9} \mathrm{~B}$ transitions directly to the ground state of ${ }^{12} \mathrm{C}$, the neutron will be energetic enough to liberate a neutron from a deuteron. For $5.85 \mathrm{MeV}$ neutrons, the $(\mathrm{n}, 2 \mathrm{n})$ cross section on deuterium has been measured at $61 \mathrm{mb}$ [89]. This is significantly larger than the $(\gamma, \mathrm{n})$ cross section on deuterium, which is $2.5 \mathrm{mb}$ for a $4.0 \mathrm{MeV} \gamma$-ray [90]. So, a significant fraction of event pairs can be n-n pairs.

To determine the number of $n-\gamma$ coincidences, we must take into account the background from n-n coincidences. So, in looking at a plot of the time to the next event for all events in the run, we must take into account the fact that the background consists of both the relatively flat exponential due to the source decay rate and a 5 msec exponential component from n-n and n- $\gamma$ pairs. We can obtain the shape of the background signal from looking at the time to the next event after a neutron, shown in Figure 6.5. Essentially this tells us how often we expect to see an n-n pair. Figure 6.6 shows a plot of the time to the next event for all events in a specific AmBe source run. It has been fit to an exponential plus the background shape. The exponential fit yielded an exponential decay constant of $5.2 \mathrm{msec}$, in good agreement with the expected lifetime of neutrons in the salted $\mathrm{D}_{2} \mathrm{O}$. The area of the exponential fit reflects the number of observed n- $\gamma$ coincidences in the data. So, the numerator in Equation 6.2 has now been obtained.

\subsection{Efficiency vs Radius}

Using the procedure outlined in the previous sections, it is possible to estimate the neutron detection efficiency for $n_{\text {eff }}>24$ hits and $R_{f i t}<725 \mathrm{~cm}$ for a given source run, based on Equation 6.2. As was mentioned above, the MC was used to provide the $4.4 \mathrm{MeV}$ 


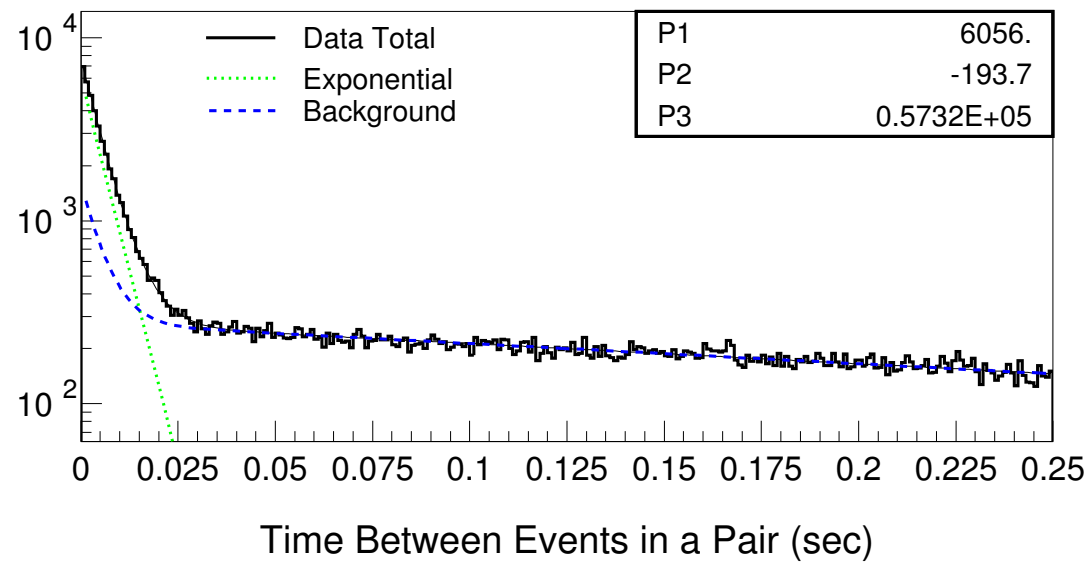

Figure 6.6: The time to the next event for all events in an AmBe source run located near the center of the detector. It has been fit to an exponential $\left(p_{1} \times e^{p_{2} t}\right)$ plus a background (with a normalization of $p_{3}$ ) that was derived from looking at the time to the next event after neutrons. The fit returns values for $p_{1}, p_{2}$, and $p_{3}$.

$\gamma$-ray response in the extraction of the number of $\gamma$-rays. The uncertainty on the energy scale for the MC is $0.8 \%$. To address this systematic, the MC was also shifted by $\pm 0.8 \%$ and the resulting uncertainty on the neutron capture efficiency was added in quadrature with the statistical error.

Figure 6.7 shows the results obtained for the neutron detection efficiency for data and MC at several different source locations. Unfortunately, the AmBe source was deployed in a very limited number of locations and the statistics at each location are low due to the low source rate.

In [91], a semi-empirical model of the neutron detection efficiency as a function of radius is given as

$$
\epsilon(s)=\epsilon_{0}-\frac{\alpha}{\beta+\left(1-\frac{s}{R}\right)^{2}}
$$




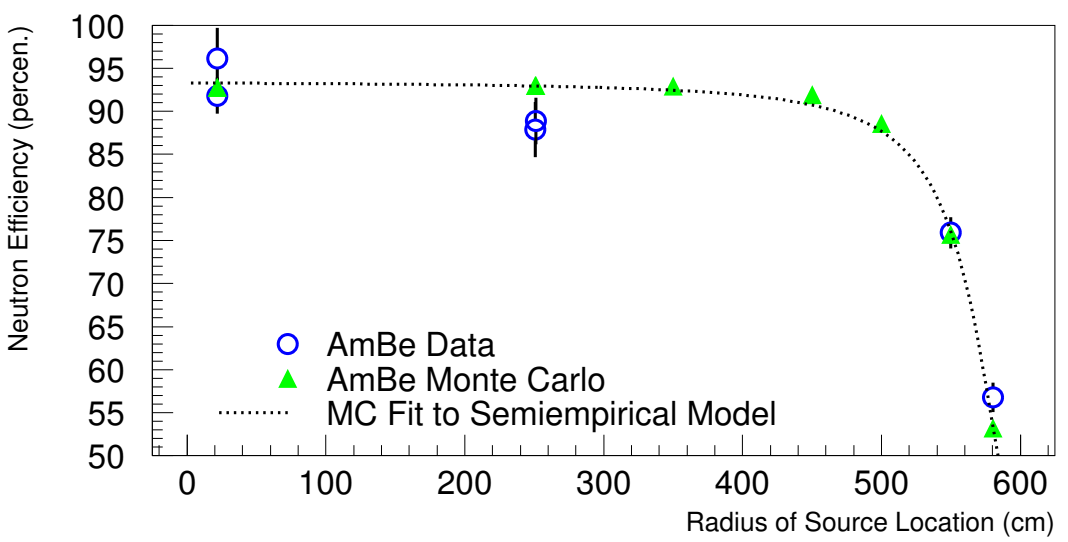

Figure 6.7: The neutron detection efficiency for $n_{\text {eff }}>24$ hits, and $R_{f i t}<725 \mathrm{~cm}$ for AmBe data and Monte Carlo simulations. The efficiency for each source run was determined using Equation 6.2, and the error bars here include a systematic uncertainty on the MC energy scale for $4.4 \mathrm{MeV} \gamma$-rays. The Monte Carlo simulations fit to Equation 6.3 is plotted here.

Here $\mathrm{R}$ is $600 \mathrm{~cm}$, and $\epsilon_{0}, \alpha$, and $\beta$ are free parameters with $\epsilon_{0}$ representing the neutron detection efficiency for an infinite volume. Using this expression and integrating it over a sphere of radius $\mathrm{R}$ gives,

$$
\epsilon_{n}=\epsilon_{0}-3 \alpha \Phi(\beta)
$$

with

$$
\Phi(\beta)=1+\ln \left(\frac{\beta}{1+\beta}\right)+\frac{1-\beta}{\sqrt{\beta}} \tan ^{-1}\left(\frac{1}{\sqrt{\beta}}\right)
$$

The points in Figure 6.7 have been fit to Equation 6.3, and the values obtained for the parameters $\alpha, \beta$, and $\epsilon_{0}$ have been inserted into Equation 6.4 to obtain the efficiency averaged over the entire volume. The values obtained are summarized in Table 6.1. Though the data and $\mathrm{MC}$ are in good agreement with each other, the statistical uncertainty on the value obtained from the data is $\pm_{0.036}^{0.031}$. Since the source position is only accurate to $\sim \pm 5$ 
cm, Table 6.1 also includes the integrated neutron efficiency obtained when the source positions are shifted radially inward or outward by $\pm 5 \mathrm{~cm}$. The uncertainty from the source location has been added in quadrature with the statistical error to obtain a final value of $0.814 \pm_{0.038}^{0.033}$ for the detection efficiency using source data with $n_{\text {eff }}>24, \mathrm{R}_{f i t}<725 \mathrm{~cm}$. The fractional uncertainty on this value is $\pm_{5.1}^{4.1} \%$

\begin{tabular}{|c|c|c|}
\hline \hline Type & Efficiency & Stat. Uncert. \\
\hline \hline MC & 0.820 & \pm 0.0032 \\
\hline Data (nominal source position) & 0.814 & $\pm_{0.036}^{0.031}$ \\
\hline Data (source R shifted out 5 cm) & 0.825 & $\pm_{0.030}^{0.027}$ \\
\hline Data (source R shifted in 5 cm) & 0.801 & $\pm_{0.044}^{0.035}$ \\
\hline \hline Data (combined) & 0.814 & $\pm_{0.038}^{0.033}$ \\
\hline
\end{tabular}

Table 6.1: Integrated neutron detection efficiency for $n_{\text {eff }}>24, \mathrm{R}_{\text {fit }}<725 \mathrm{~cm}$

\subsection{Summary of Neutron Detection Efficiency}

The neutron detection efficiencies given in Table 6.1 represent the neutron detection efficiency for events with $n_{\text {eff }}>24$ hits and $R_{f i t}<725 \mathrm{~cm}$. In the final analysis, we need to known the neutron detection efficiency for energy $>5.50 \mathrm{MeV}$ and $R_{f i t}<550 \mathrm{~cm}$. We can use the Monte Carlo simulation to give us the final detection efficiency, however we must take into account the uncertainty on the measurement made with the data. Monte Carlo simulations of the NC signal at 100 times the expected signal from the Standard Solar Model have been performed for each run in the final dataset. These Monte Carlo simulations give a neutron detection efficiency of $0.391 \pm 0.001$ (stat.). The results from the data (in Table 6.1) give a fractional uncertainty on the neutron detection efficiency is $\pm_{5.1}^{4.1}$ 
\%. This gives a value for the neutron detection efficiency of $0.391 \pm_{0.020}^{0.016}$.

It is also the case that the uncertainty on the energy threshold and the fiducial volume (due to reconstruction radial pull) will have an impact on the neutron detection efficiency. However, these two factors will be accounted for later when determining the number of neutrino events in the salt dataset, and will not be included here.

For comparison purposes, the neutron detection efficiency obtained with the ${ }^{252} \mathrm{Cf}$ source for the full salt dataset is $0.398 \pm 0.005$ (stat.) $\pm_{0.010}^{0.012}[92]$, in good agreement with the value obtained here with the AmBe source.

\begin{tabular}{|c|c|}
\hline \hline Efficiency & Uncertainty \\
\hline \hline 0.391 & $\pm_{0.020}^{0.016}$ \\
\hline
\end{tabular}

Table 6.2: Integrated neutron detection efficiency for visible energy $>5.50 \mathrm{MeV}$ and $\mathrm{R}_{\text {fit }}<550 \mathrm{~cm}$. 


\section{Chapter 7}

\section{Removal of Non-Physics Events}

\subsection{Instrumental Backgrounds}

In the previous chapters, the event reconstruction, energy calibration, event isotropy, and neutron detection response of the SNO detector have been evaluated. These are the basic building blocks required to search for neutrino signatures in the data. But, before moving on to extracting the neutrino signals, we must first consider the subject of backgrounds. This chapter is devoted to instrumental backgrounds, i.e. those that are associated with artifacts of the detector system. The subsequent chapter will be devoted to the physics backgrounds expected in the detector, such as events from the intrinsic radioactivity, background neutrons, and products from muon interactions.

Unfortunately there are several classes of background events seen in the SNO detector that arise from electronic noise and static discharges. The major instrumental backgrounds are described below:

Flashing Photo-multiplier Tubes: By far most of the background events stem 
from flashing photo-multiplier tubes. Occasionally there is an electrostatic discharge in a PMT. This results in the emission of light and the deposition of a large amount of charge into the flashing photo-multiplier tube's dynode. Its neighboring channels on the electronics card will pick up some of this charge and also register hits. Then, at a time corresponding to the time that it takes light to travel across the PMT array, photo-multiplier tubes on the opposite side of the detector will see light. These events occur periodically in the detector, but large bursts are seen during periods of seismic activity or following blasting in the mine.

Neck Events: A class of events near the top of the chimney of the acrylic vessel (the "neck" of the detector) casts light down onto the photo-multiplier tubes located at the bottom of the detector. They are believed to be caused by a static discharge near the surface of the water. A set of photo-multiplier tubes was added to the top of the chimney to detect this light and veto these events.

Breakdown: When a the insulation in the connector of a photo-multiplier tube fails it can produce electrical pickup in a single electronics crate and will sometimes produce large bursts of light in the detector.

Retriggers: When a physics event fires are large number of the PMTs in the detector - such as a muon that triggers thousands of PMTs - the large amount of digital read-out activity causes cross talk between the electronics channels. This causes them to fire again, and if enough channels refire, then the detector can be triggered again. For these events, the charges on the channels will be low and the time at which the channels refire will be distributed over a broad time window. 


\subsection{Cuts to Remove Instrumental Backgrounds}

In order to remove instrumental backgrounds, we can first examine the charge, location, and timing of the photo-multiplier tube hits. Here the location can refer to the physical location of the tube in the detector, or to its location in the electronics. ${ }^{1}$ With this in mind, a series of instrumental background cuts has been developed.

\subsubsection{Level 1: Instrumental Background Cuts}

The instrumental background cuts are more fully described in [93, 94].

\section{Cuts based on the locations of the photo-multiplier tube hits}

Junk: Removes events that have multiple entries for a photo-multiplier tube or are orphans (collections of hits which could not be associated with a specific trigger).

Neck: Removes events that have at least 2 hits recorded in the "neck" photomultiplier tubes or events with one neck tube hit with a large charge and early time.

OWL: Removes events where more than 5 outward-looking tubes (i.e. muon veto) have fired.

Crate Isotropy: Removes events where more than $70 \%$ of the events are in one electronics crate and more than $80 \%$ of those are in two or fewer cards.

Flasher Geometry: Looks for a cluster of 4 or more adjacent hits. Removes the event if the average distance to the other tubes is more than $1200 \mathrm{~cm}$. Designed to cut flashing photo-multiplier tube events.

\footnotetext{
${ }^{1}$ As was described in Chapter 2, the electronics location is described by a crate, card, and channel number.
} 


\section{Cuts based on the time of the photo-multiplier tube hits ${ }^{2}$}

Retrigger $(*)$ : Removes events which are less than $5 \mu$ sec after another event.

Nhit Burst(*): Removes events when more than 6 events with over 40 hits occur within less than 4 seconds of one another.

Muon Follower(*): Tags muons (events with more than 150 hits on normal PMTs and more than five OWL tubes) and removes all events for the next 20 seconds. More will be said about muon followers in the next chapter.

Missed Muon Follower(*): Removes all events within 250 msec after an event with over 150 hits (in case the muon was missed by the tag in the Muon Follower cut)

In-Time Channel: Removes events where less than $60 \%$ of the hits are in a $93 \mathrm{~ns}$ wide time window.

Fitterless Time Spread: Looks at tubes that are within 3 meters of each other in space and 25 nsec of each other in time. Removes the event if the median pairwise tube-to-tube time difference is greater than 6.8 nsec.

\section{Cuts based on the charges of the photo-multiplier tube hits}

Analog Measurement Board: Looks at the sum of all the PMT pulses for an event. If the integral or peak is more than $3.7 \sigma$ away from the expected value for an event with that number of photo-multiplier tube hits, the event is removed.

ESUM: If the event only triggered the detector because it was above the charge threshold, but did not satisfy any trigger conditions for the number of hit photo-multiplier

\footnotetext{
${ }^{2}$ The first four cuts, denoted by a $(*)$, cut out blocks of time in the data stream. The SNO data livetime is calculated with the time lost from these cuts taken into account.
} 


\begin{tabular}{|c|c|}
\hline Electrons (\%) & Neutrons (\%) \\
\hline $99.63_{-0.15}^{+0.09}$ & $99.60_{-0.15}^{+0.09}$ \\
\hline
\end{tabular}

Table 7.1: Level 1 cut acceptance for events within the fiducial volume and above $5.50 \mathrm{MeV}$ (visible energy), from [95].

tubes, then it is removed (i.e. the event has a lot of charge deposited but only a small number of photo-multiplier tubes).

OWL Trigger: Removes events where there is a large light deposition in the outward-looking PMTs.

Q vs T: Removes events where the tube with the largest charge in the event is well above the average charge expected for an event and this tube also fired more than 60 ns before the median tube time.

Q Cluster: Removes events that have a cluster of hits in of 4 out of 5 adjacent channels, and at least one of the channels in the cluster has a high charge.

The acceptance of these cuts has been evaluated for electrons and neutrons in [95].

The results, determined with the tagged ${ }^{16} \mathrm{~N},{ }^{8} \mathrm{Li}$, and burst filtered ${ }^{252} \mathrm{Cf}$ sources, are summarized in Table 7.1. As was mentioned above, this acceptance does not include the Retrigger, Nhit Burst, Muon Follower, and Missed Muon Follower cuts because the effect of these cuts is taken into account when determining the livetime for the dataset.

\subsubsection{Level 2: High Level Cuts}

In addition to the instrumental background cuts described in the previous section, there is a second level of cuts that depend on event reconstruction. The purpose of these cuts is to remove poorly reconstructed events and to reject events that do not resemble 
Čerenkov light. The two Level 2 cuts are:

In-Time Ratio: The in-time ratio (or ITR) is the number of hits that occur within a -2.5 to 5.0 ns window (relative to the mean arrival time of direct light hits) divided by the total number of hits in the events. Events are required to have ITR $>0.55$. In other words, at least $55 \%$ of the photo-multiplier tube hits must be prompt.

$\theta_{\mathbf{i j}}: \theta_{i j}$ has already been extensively discussed in Chapter 5. A cut requiring events to have $\theta_{i j}>0.75$ is also applied to the data. This removes events in which the hits are clustered into a small region of the detector, which might be a sign that the events are not arising from Čerenkov light.

Since both of these cuts depend on the reconstructed vertex, the cut acceptance must be evaluated for the reconstruction algorithm used in this dissertation. To determine the acceptance, we can utilize the ${ }^{16} \mathrm{~N}$ and ${ }^{252} \mathrm{Cf}$ source data. The acceptance of the Level 2 cuts is determined by the number of good physics events that passed the Level 2 cuts divided by the number of events that passed the Level 1 cuts from the same data sample.

For the ${ }^{16} \mathrm{~N}$ source data, the events have been required to have a beta tag present. Figure 7.1 shows the acceptance above $5.50 \mathrm{MeV}$ for ${ }^{16} \mathrm{~N}$ runs near the center of the detector as a function of time. The points have been fit to a line. Taking half of the difference in the fit at the start of the dataset and the end of the dataset gives an uncertainty of $\pm 0.001 \%$ in the acceptance due to time variation. Figures 7.2 and 7.3 show the acceptance as a function of the reconstructed event radius for data and MC. Though some differences are seen at large radii, the volume weighted average of the data over the fiducial volume of $\mathrm{r}<550 \mathrm{~cm}$ (or $\mathrm{r}^{3} / 600 \mathrm{~cm}^{3}<0.77$ ) agrees with the average from the MC to within $0.002 \%$. 


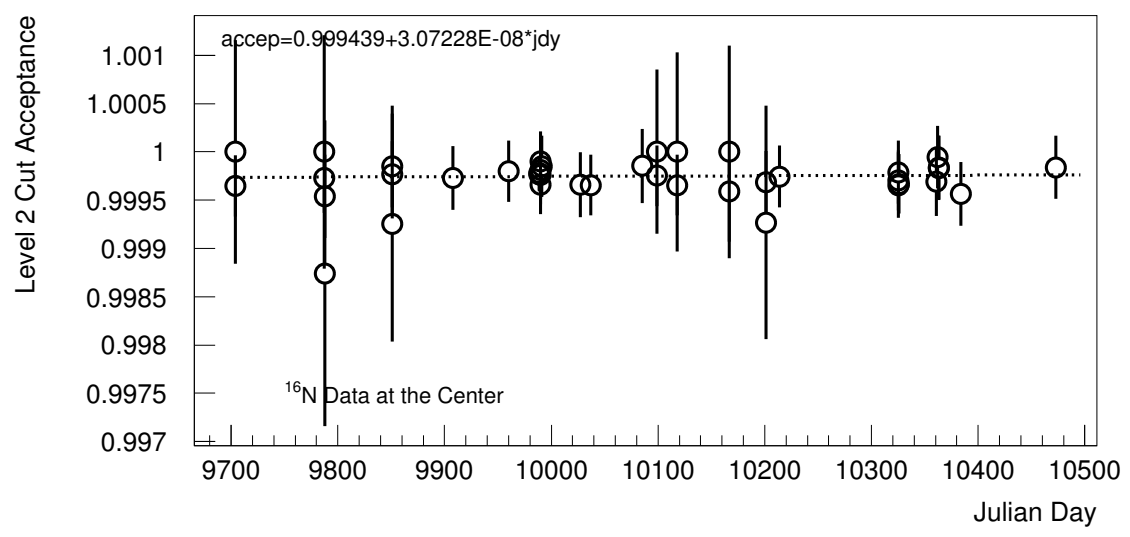

Figure 7.1: Acceptance of the Level 2 cuts above $5.50 \mathrm{MeV}$ and within $550 \mathrm{~cm}$ as a function of time for ${ }^{16} \mathrm{~N}$ source data near the center of the detector

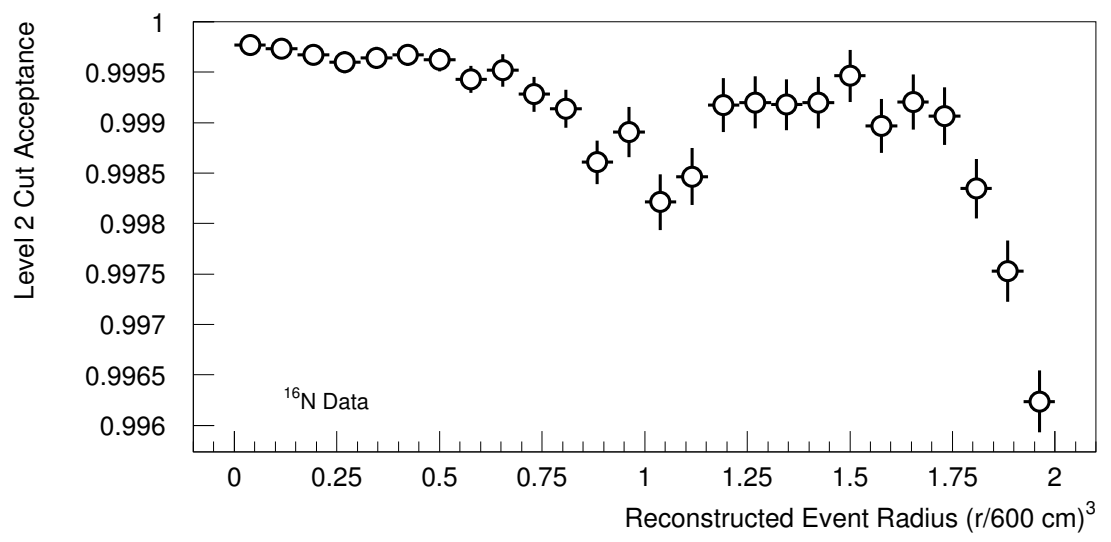

Figure 7.2: Level 2 cut acceptance above $5.50 \mathrm{MeV}$ as a function of reconstructed radius for ${ }^{16} \mathrm{~N}$ source data. 


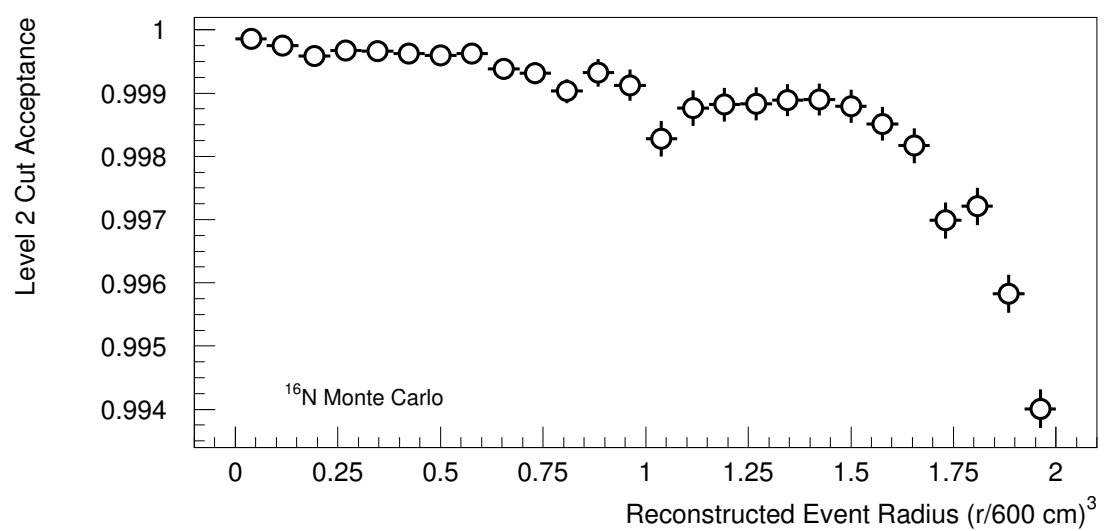

Figure 7.3: Level 2 cut acceptance above $5.50 \mathrm{MeV}$ as a function of reconstructed radius for ${ }^{16} \mathrm{~N}$ source $\mathrm{MC}$.

We also need to look at the acceptance for ${ }^{252} \mathrm{Cf}$ source data and Monte Carlo simulations to verify the performance for neutrons. Figures 7.4 and 7.5 show the Level 2 cut acceptance for ${ }^{252} \mathrm{Cf}$ source data and Monte Carlo simulations as a function of the reconstructed position. For the data, the Level 1 cuts have been applied, and the events are required to occur as part of a burst of 3 to 10 events, with the first event being excluded (since it can be $\gamma$-rays associated with the fission). The volume weighted average value of the data agrees with that from the Monte Carlo simulations to within $0.01 \%$ inside of the fiducial volume.

The ${ }^{252} \mathrm{Cf}$ and ${ }^{16} \mathrm{~N}$ source data and $\mathrm{MC}$ tell us the systematic uncertainties associated with the Level 2 cut acceptance. However, the acceptance will vary somewhat with the energy of the event. Events with fewer hits will tend to have a smaller acceptance since the $\theta_{i j}$ and ITR distributions will have longer tails. This is a concern for the elastic-scattering 


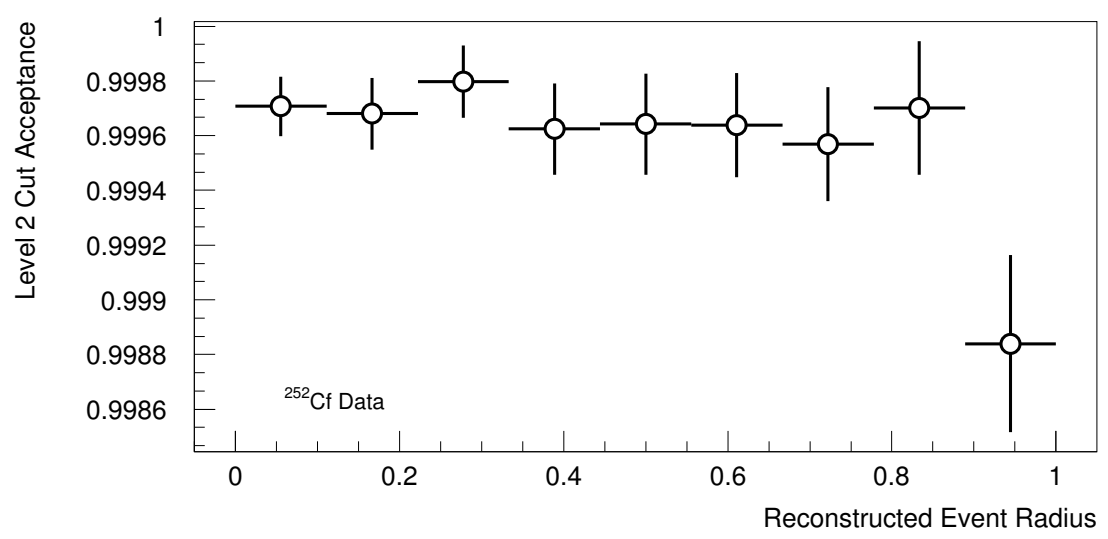

Figure 7.4: Level 2 cut acceptance above $5.50 \mathrm{MeV}$ as a function of reconstructed radius for ${ }^{252} \mathrm{Cf}$ source data.

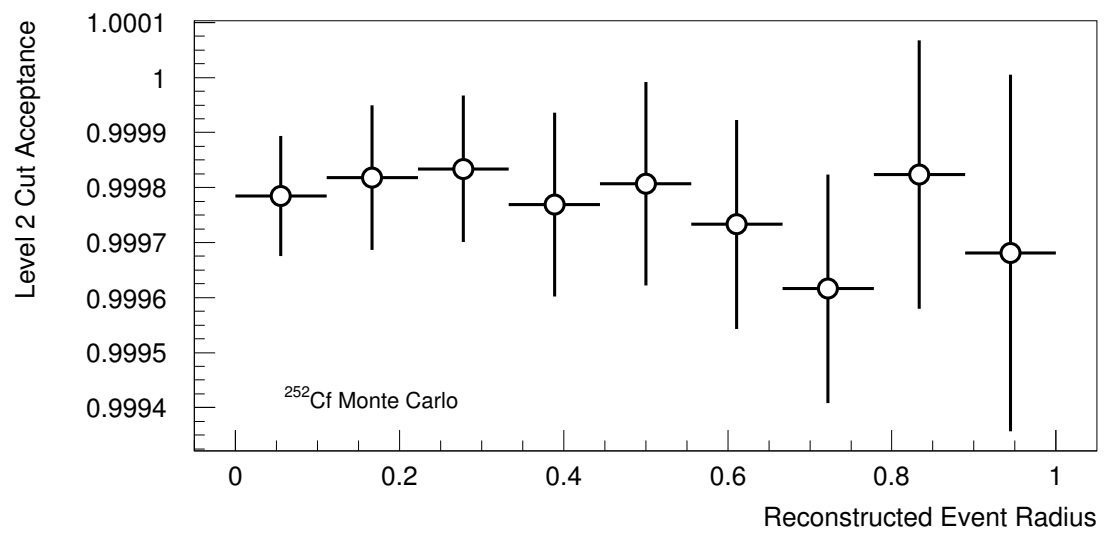

Figure 7.5: Level 2 cut acceptance above $5.50 \mathrm{MeV}$ as a function of reconstructed radius for ${ }^{252} \mathrm{Cf}$ source $\mathrm{MC}$. 


\begin{tabular}{|c|c|c|c|}
\hline Signal & CC (\%) & ES (\%) & NC (\%) \\
\hline \hline Acceptance & 99.975 & 99.948 & 99.984 \\
\hline \hline Stat. Uncert. & 0.006 & 0.018 & 0.012 \\
\hline Time Variation & 0.001 & 0.001 & 0.001 \\
\hline Agreement of MC with Data & 0.002 & 0.002 & 0.010 \\
\hline \hline Total Uncertainty & 0.006 & 0.018 & 0.016 \\
\hline
\end{tabular}

Table 7.2: Level 2 cut acceptance for events within the fiducial volume and above $5.50 \mathrm{MeV}$ (visible energy).

signal, since the ES events have a lower average energy than the $\mathrm{CC}, \mathrm{NC}$, or ${ }^{16} \mathrm{~N}$ source data. For this reason, we will use the Monte Carlo simulations of the neutrino signals to give us the final acceptance numbers, since these will take into account the expected energy spectrum for each neutrino signal. A limit on the temporal variation of the acceptance comes from the ${ }^{16} \mathrm{~N}$ source, and the ${ }^{16} \mathrm{~N}$ and ${ }^{252} \mathrm{Cf}$ source data and $\mathrm{MC}$ give us an idea of how well the data agrees with the Monte Carlo simulations. The acceptance values and systematic uncertainties are summarized in Table 7.2.

\subsection{Purity of the Dataset}

The purity of the dataset has been determined in [95], where a bifurcated analysis technique was used. Suppose that there are two orthogonal sets of cuts designed to remove background events from the data. There are four possible outcomes for events, depicted in Figure 7.6. We can write down the following relations to describe the number of events 
with each of the four possible outcomes

$$
\begin{gathered}
N=S+\beta \\
a=\epsilon_{1} \epsilon_{2} S+\kappa_{1} \kappa_{2} \beta \\
a+b=\epsilon_{2} S+\kappa_{2} \beta \\
a+d=\epsilon_{1} S+\kappa_{1} \beta
\end{gathered}
$$

where $\mathrm{N}$ is the total number of events, $\mathrm{S}$ is the number of signal events, $\beta$ is the number of background events, $\epsilon_{1}$ and $\epsilon_{2}$ represent the acceptance of cuts 1 and 2 for the signal, $\kappa_{1}$ and $\kappa_{2}$ are the acceptance of cuts 1 and 2 for the background. The regions a,b,c, and d are defined in Figure 7.6. In these four equations, N, a, b, and d are known by counting up the number of events in the dataset and the number that pass or fail cut 1 and cut 2 . From a study of the cut acceptance, $\epsilon_{1}$ and $\epsilon_{2}$ can be determined. Therefore, by arranging these relations, $\kappa_{1} \kappa_{2} \beta$, or the number of background events remaining in the sample after cuts 1 and 2 are applied can be determined. This analysis assumes that there is only one type of background and is only sensitive to backgrounds that the cuts were designed to remove. It also requires that the cuts are orthogonal to each other.

Before performing the analysis, the junk cut, the muon follower cut, retrigger, nhit burst, and ITC cut, energy threshold and fiducial volume cuts were applied. Cut 1 was then composed of the remaining Level 1 cuts (see Section 7.2.1) and the Cut 2 was composed of the Level 2 cuts (see Section 7.2.2). The Level 1 cuts are based on photo-multiplier tube information like the charge, position and timing, while the Level 2 cuts are based on reconstruction, which is only weakly correlated with the individual photo-multiplier tube information. The result obtained for the contamination of the dataset by this bifurcated technique was $<3$ events at the $95 \%$ confidence level [95]. 


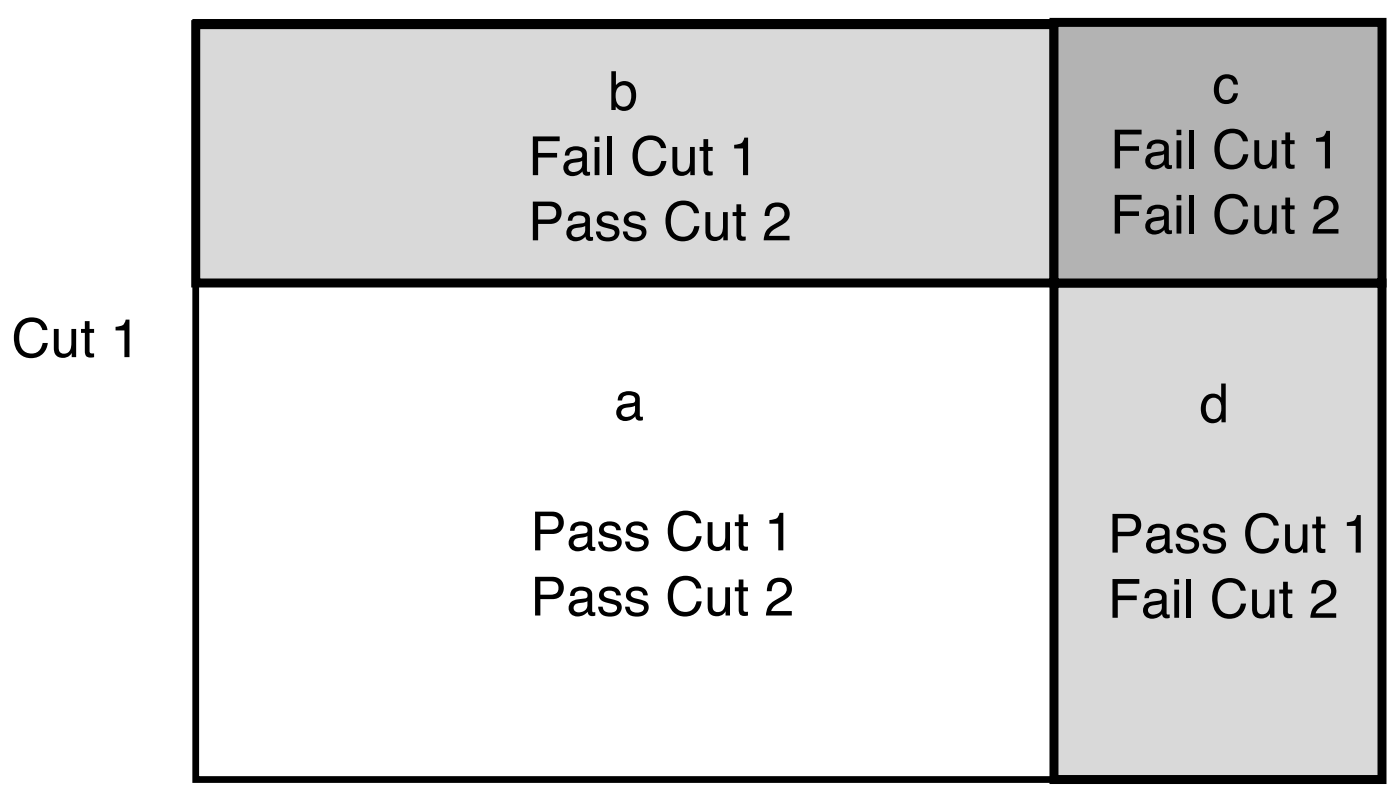

Cut 2

Figure 7.6: The definitions of regions a,b,c, and d in Equations 7.1.

\begin{tabular}{|c|}
\hline Instrumental Background Events \\
\hline$<3$ events \\
\hline \hline
\end{tabular}

Table 7.3: Estimated instrumental background contamination remaining the the dataset within the fiducial volume and above $5.50 \mathrm{MeV}$ (visible energy) following the application of the Level 1 and Level 2 cuts [95]. 


\section{Chapter 8}

\section{Physics Backgrounds}

\subsection{Introduction to Physics Backgrounds}

In addition to the instrumental backgrounds in Chapter 7, there are a large number of physics processes that can also contribute backgrounds to the neutrino signal. The dominant source of background is the low-energy $\beta$ and $\gamma$-rays from $U$ and Th inside the detector (Section 8.2) and in the PMTs and PSUP (Section 8.3). Higher energy $\gamma$-rays from the steel in the PSUP and the rock wall can also enter the fiducial volume (Section 8.4). Though the cosmic-ray muon flux in SNO is greatly reduced by the rock overburden, muons will still enter the detector, where they can leave behind neutrons and other radioactive products (Section 8.5). A class of highly isotropic events that reconstruct near the AV has been observed and it is necessary to estimate how many of these events remain inside the fiducial volume (Section 8.6). Contributions from other miscellaneous process, such as atmospheric neutrinos and $\alpha$-induced interactions, have also been estimated (Section 8.7). 


\subsection{Internal U and Th Backgrounds}

The primary sources of radioactive backgrounds in the SNO detector are ${ }^{232} \mathrm{Th}$ and ${ }^{238} \mathrm{U}$ and their decay chain progenies. Figure 8.1 shows the ${ }^{232} \mathrm{Th}$ decay chain to stable ${ }^{208} \mathrm{~Pb}$. The vast majority of the decay steps produce alphas (which will not produce Cerenkov radiation in $\mathrm{D}_{2} \mathrm{O}$ unless they are above a $\mathrm{GeV}$ in energy) and low-energy betas, which are significantly below the detector threshold. The nucleus of concern here is ${ }^{208} \mathrm{Tl}$, which can yield a $\beta$ up to $5 \mathrm{MeV}$ and will always produce a $2.6 \mathrm{MeV} \gamma$-ray. Recall that $\gamma$ rays above $2.2 \mathrm{MeV}$ are capable of photodisintegrating deuterium, producing a free neutron. So, ${ }^{208} \mathrm{Tl}$ is a background to the Čerenkov signal for CC and ES events and to the neutron signal.

Figure 8.2 shows the decay chain for ${ }^{238} \mathrm{U}$, where the major concern is ${ }^{214} \mathrm{Bi}$, which can produce a $3.3 \mathrm{MeV} \beta$ and produces a $2.45 \mathrm{MeV} \gamma$-ray in $1.5 \%$ of its decays. The decay schemes for both ${ }^{208} \mathrm{Tl}$ and ${ }^{214} \mathrm{Bi}$ are shown in Figure 8.3.

As will be described below, the levels of $\mathrm{U}$ and Th in the detector components have been determined by a variety of methods. Monte Carlo simulations of the $\mathrm{U}$ and $\mathrm{Th}$ chain backgrounds in various regions of the detector have been performed.

\subsection{1 $\quad{ }^{238} \mathrm{U}$ and ${ }^{232} \mathrm{Th}$ Levels in Acrylic}

For the acrylic vessel, there are two sources of $U$ and Th activity. The first is the intrinsic radioactivity in the bulk of the acrylic. The second is activity on the surface of the AV due to mine dust which settled on the surface during construction. In [96], the amounts of surface and bulk $\mathrm{U}$ and Th was estimated. Table 8.1 summarizes the results. From the 


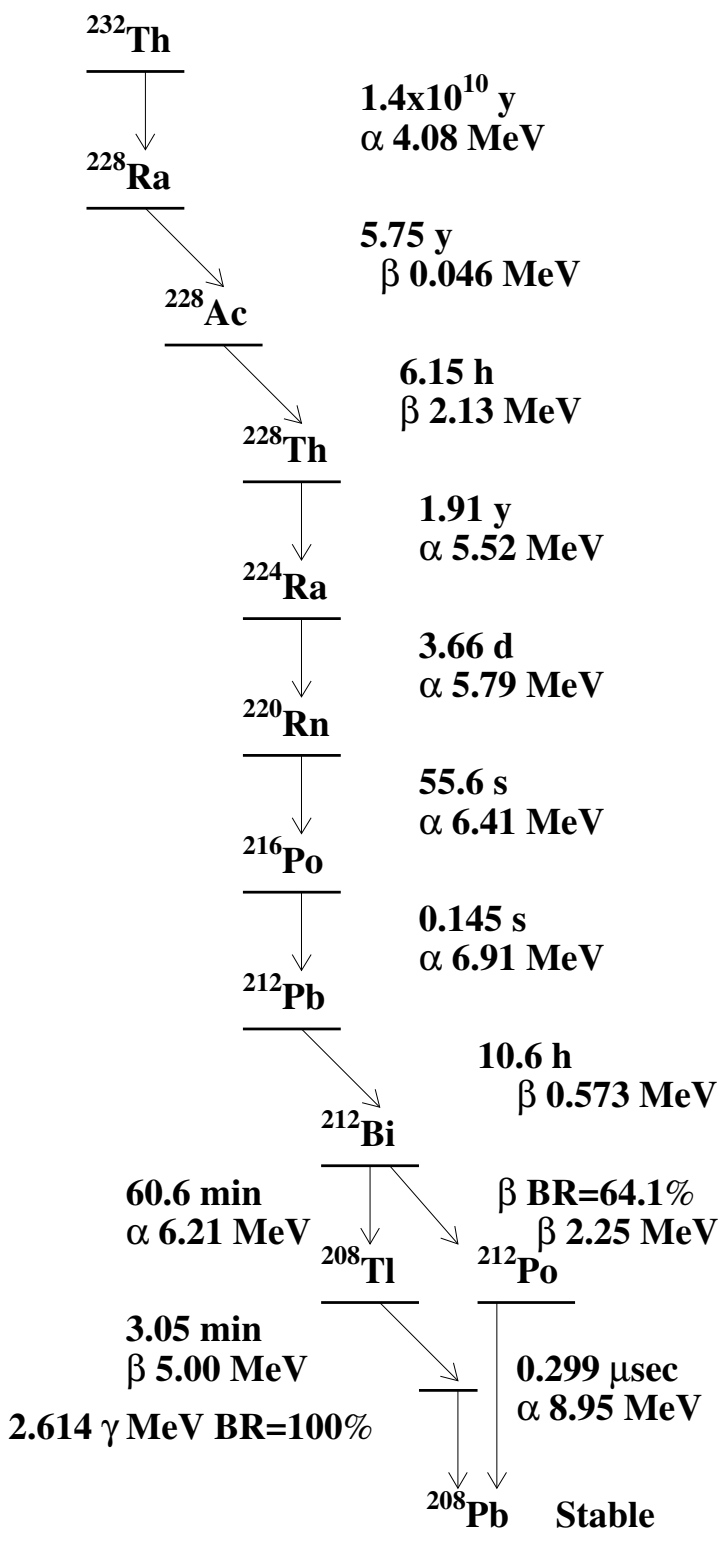

Figure 8.1: The ${ }^{232}$ Th decay chain, based on a figure in [34] and with data from [60]. Printed are the half-live, and the $\beta$ and $\alpha$ Q-values. $\gamma$-rays below $2.2 \mathrm{MeV}$ are not included. 


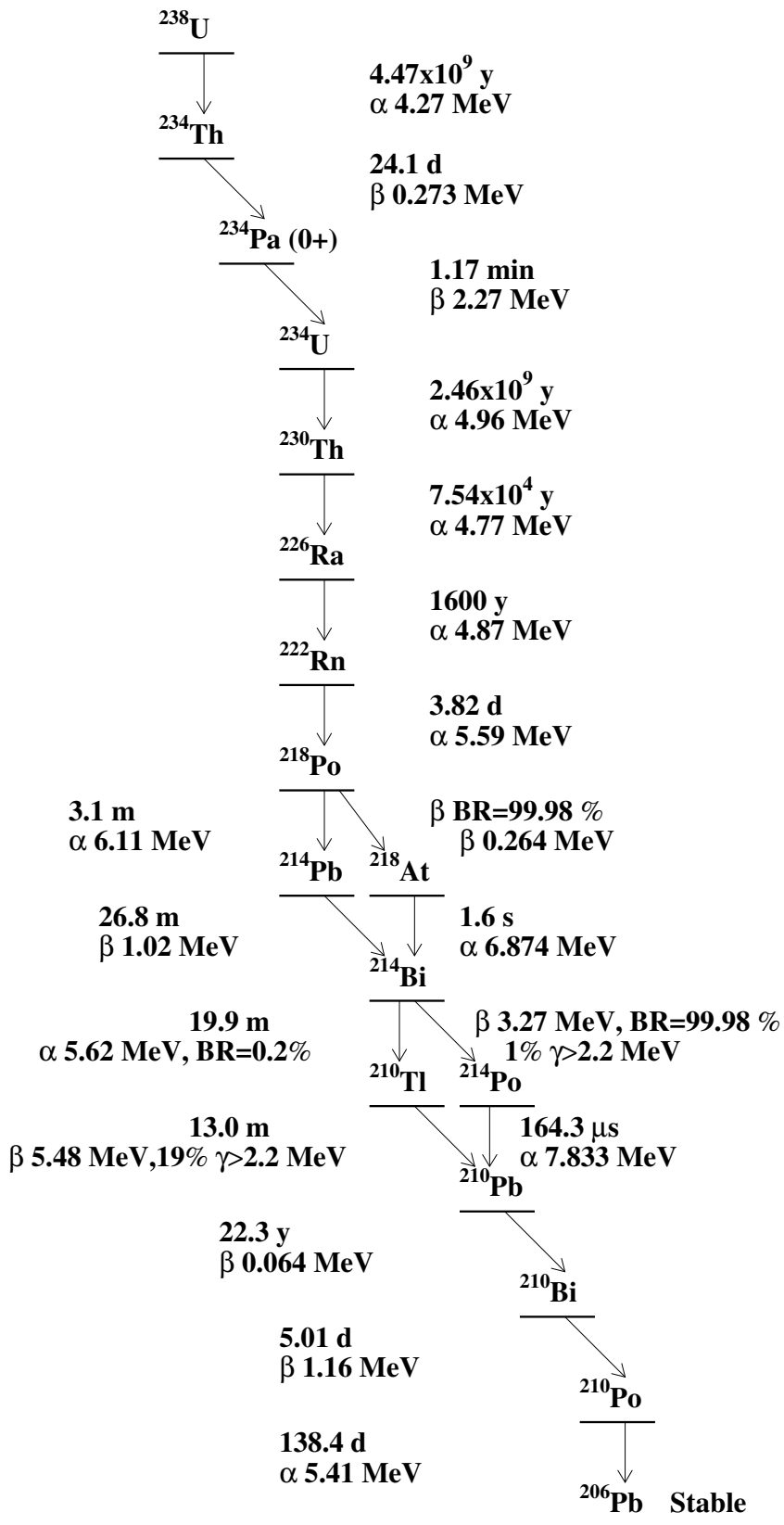

Figure 8.2: The ${ }^{238} \mathrm{U}$ decay chain, based on a figure in [34] and with data from [60]. Printed are the half-lives, and the $\beta$ and $\alpha$ Q-values. $\gamma$-rays below $2.2 \mathrm{MeV}$ are not included. 

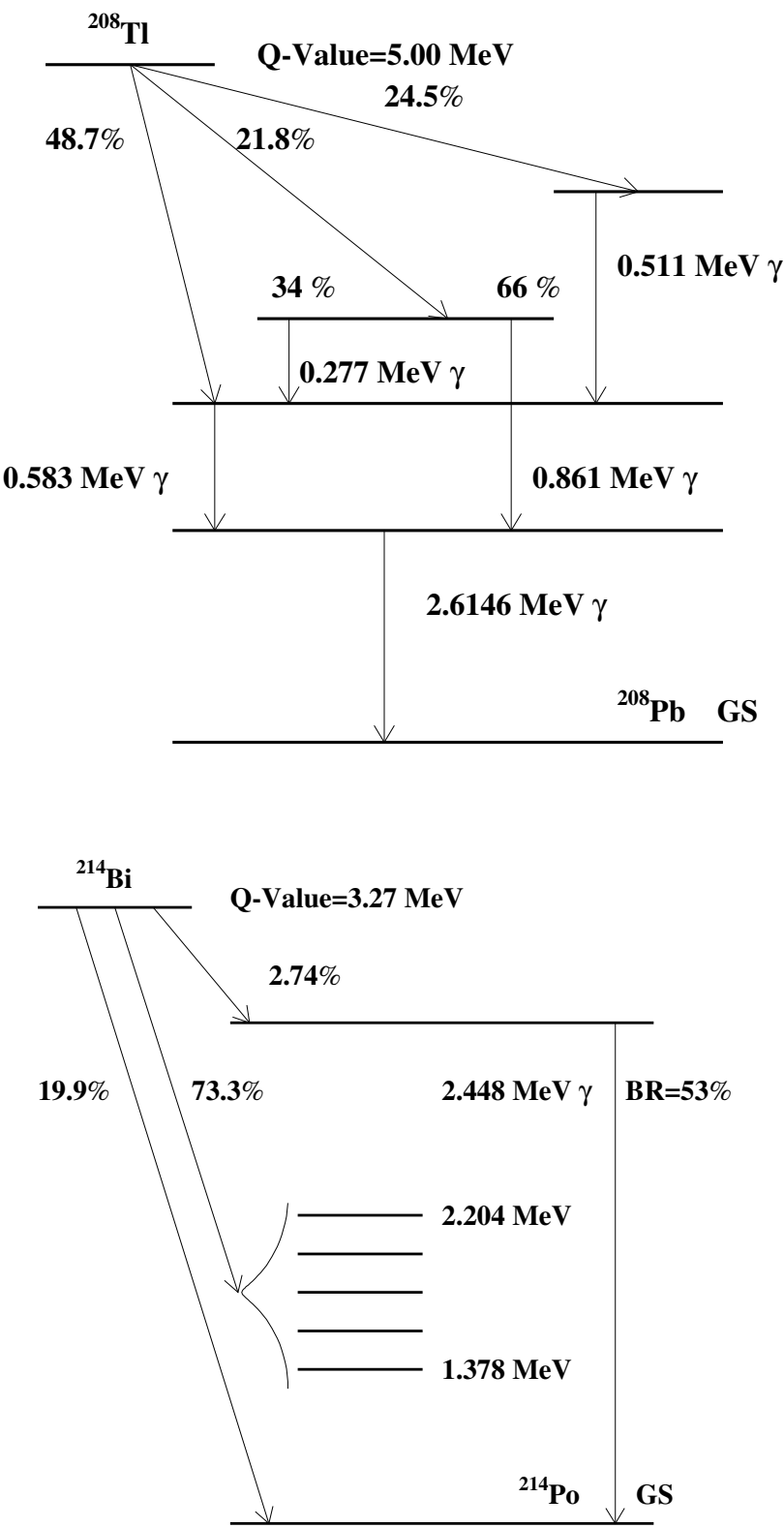

Figure 8.3: Simplified ${ }^{208} \mathrm{Tl}$ and ${ }^{214} \mathrm{Bi}$ decay schemes based on a figure in [34] and with data from [60]. 


\begin{tabular}{|c|c|c|c|c|}
\hline Medium & Source & Amount $(\mu \mathrm{g})$ & Progeny & Progeny Rate $\left(\mathrm{s}^{-1}\right)$ \\
\hline AV Surface & ${ }^{238} \mathrm{U}$ & $0.34 \pm 0.06$ & ${ }^{214} \mathrm{Bi}$ & $0.0042 \pm 0.0007$ \\
\hline AV Surface & ${ }^{232} \mathrm{Th}$ & $1.83 \pm 0.25$ & ${ }^{208} \mathrm{Tl}$ & $0.00268 \pm 0.00037$ \\
\hline AV Bulk & ${ }^{238} \mathrm{U}$ & $15 \pm 15$ & ${ }^{214} \mathrm{Bi}$ & $0.19 \pm 0.19$ \\
\hline AV Bulk & ${ }^{232} \mathrm{Th}$ & $7.5 \pm 1.3$ & ${ }^{208} \mathrm{Tl}$ & $0.011 \pm_{0.0015}^{0.0025}$ \\
\hline \hline AV Total & ${ }^{238} \mathrm{U}$ & $15.3 \pm 15$ & ${ }^{214} \mathrm{Bi}$ & $0.194 \pm 0.19$ \\
\hline AV Total & ${ }^{232} \mathrm{Th}$ & $9.33 \pm \pm_{1.3}^{1.7}$ & ${ }^{214} \mathrm{Bi}$ & $0.0137 \pm_{0.0015}^{0.0025}$ \\
\hline \hline
\end{tabular}

Table 8.1: Concentrations of $\mathrm{U}$ and Th on the AV surface and the AV bulk from [96].

amounts, it is possible to estimate the decay rate for the progenies of interest $\left({ }^{214} \mathrm{Bi}\right.$ and ${ }^{208} \mathrm{Tl}$ ), and these have also been included in Table 8.1.

\subsection{2 ${ }^{238} \mathrm{U}$ and ${ }^{232} \mathrm{Th}$ Levels in $\mathrm{D}_{2} \mathrm{O}$ and $\mathrm{H}_{2} \mathrm{O}$}

SNO uses two techniques to determine the ${ }^{238} \mathrm{U}$ and ${ }^{232} \mathrm{Th}$ levels in $\mathrm{H}_{2} \mathrm{O}$ and $\mathrm{D}_{2} \mathrm{O}$.

In-Situ: This technique looks for signatures of ${ }^{214} \mathrm{Bi}$ and ${ }^{208} \mathrm{Tl}$ with the PMT hit pattern to determine the levels of ${ }^{238} \mathrm{U}$ and ${ }^{232} \mathrm{Th}$ in the data. Low-energy monitoring windows in the $\mathrm{D}_{2} \mathrm{O}$ and $\mathrm{H}_{2} \mathrm{O}$ are examined and a maximum likelihood fit to the $\theta_{i j}$ distribution determines the relative amounts of ${ }^{208} \mathrm{Tl}$ and ${ }^{214} \mathrm{Bi} .\left({ }^{208} \mathrm{Tl}\right.$ tends to have a more isotropic light pattern since it always produces a $\beta$ and at least two $\gamma$-rays when it decays.)

Ex-Situ: This technique consists of a variety of chemical assays to determine the concentrations of ${ }^{232} \mathrm{Th}$ and ${ }^{238} \mathrm{U}$ decay chain products. There are actually three different chemical assays that are performed. In the first, the water is passed over $\mathrm{MnOx}$ coated beads, which collect Ra. The Ra then decays to Rn and then to Po, which is detected by $\alpha$ counters. The abundances of various alphas reveal the $\mathrm{U}$ and $\mathrm{Th}$ concentrations. In the second process, the water is passed through hydrous titanium oxide (HTiO) coated fibers. 


\begin{tabular}{|c|c|c|c|c|}
\hline Medium & Source & Concen. $(\mathrm{g} / \mathrm{g})$ & Progeny & Progeny Rate $\left(\mathrm{s}^{-1}\right)$ \\
\hline $\mathrm{D}_{2} \mathrm{O}$ & ${ }^{238} \mathrm{U}$ & $8.28 \pm_{2.10}^{1.38} \times 10^{-15}$ & ${ }^{214} \mathrm{Bi}$ & $0.103 \pm_{0.026}^{00.017}$ \\
\hline $\mathrm{D}_{2} \mathrm{O}$ & ${ }^{232} \mathrm{Th}$ & $1.11 \pm 0.69 \times 10^{-15}$ & ${ }^{208} \mathrm{Tl}$ & $0.00162 \pm 0.00101$ \\
\hline $\mathrm{H}_{2} \mathrm{O}$ & ${ }^{238} \mathrm{U}$ & $20.2 \pm 6.1 \times 10^{-14}$ & ${ }^{214} \mathrm{Bi}$ & $4.27 \pm 1.29$ \\
\hline $\mathrm{H}_{2} \mathrm{O}$ & ${ }^{232} \mathrm{Th}$ & $5.45 \pm 2.05 \times 10^{-14}$ & ${ }^{208} \mathrm{Tl}$ & $0.136 \pm 0.051$ \\
\hline
\end{tabular}

Table 8.2: Concentration of $\mathrm{U}$ and $\mathrm{Th}$ in the $\mathrm{H}_{2} \mathrm{O}$ and $\mathrm{D}_{2} \mathrm{O}$ from $[97,98]$.

The fibers are then brought to the surface, the Ra is extracted chemically, and a delayed coincidence counting of the $\mathrm{Bi}$ and subsequent Po decays determines the concentrations of ${ }^{224} \mathrm{Ra}$ (Th chain) and ${ }^{226} \mathrm{Ra}$ (U chain). Since ${ }^{222} \mathrm{Rn}$ from air in the laboratory can enter into system and look like U chain activity, a third technique involves a direct assay of the $\mathrm{Rn}$ in the water by degassing the water, collecting $\mathrm{Rn}$ and alpha counting it for ${ }^{222} \mathrm{Rn}$.

The results of these two techniques are summarized in Table 8.2 [97, 98]. Also listed in the table is the decay rate of the progeny nucleus $\left({ }^{208} \mathrm{Tl}\right.$ or $\left.{ }^{214} \mathrm{Bi}\right)$.

\subsubsection{Summary of backgrounds from Internal $U$ and Th}

Simulations of ${ }^{208} \mathrm{Tl}$ and ${ }^{214} \mathrm{Bi}$ decays distributed uniformly throughout the $\mathrm{H}_{2} \mathrm{O}$ $\mathrm{D}_{2} \mathrm{O}$ and $\mathrm{AV}$ bulk have been performed. By scaling them to the appropriate decay rates, given in Tables 8.1 and 8.2, one can estimate the number of Čerenkov background events expected within the fiducial volume of $550 \mathrm{~cm}$ and above $5.5 \mathrm{MeV}$ of visible energy in the entire 391.4 day dataset. The data cleaning cuts (both Level 1 and Level 2) have also been applied. Systematic uncertainties due to the energy scale, energy resolution, and fiducial volume have been included. The Čerenkov backgrounds are summarized in Table 8.3. Since the number of events is so small, they will be treated as a systematic uncertainty on the 


\begin{tabular}{|c|c|c|}
\hline Medium & Source & Number of Background Events \\
\hline $\mathrm{D}_{2} \mathrm{O}$ & ${ }^{238} \mathrm{U}$ & $1.03 \pm_{0.37}^{0.95}$ \\
\hline $\mathrm{D}_{2} \mathrm{O}$ & ${ }^{232} \mathrm{Th}$ & $0.219 \pm_{0.145}^{0.300}$ \\
\hline $\mathrm{H}_{2} \mathrm{O}$ & ${ }^{238} \mathrm{U}$ & $0.856 \pm 0.501$ \\
\hline $\mathrm{H}_{2} \mathrm{O}$ & ${ }^{232} \mathrm{Th}$ & $0.204 \pm 0.156$ \\
\hline $\mathrm{AV}$ & ${ }^{238} \mathrm{U}$ & $0 \pm 0.077$ \\
\hline $\mathrm{AV}$ & ${ }^{232} \mathrm{Th}$ & $0.045 \pm 0.046$ \\
\hline \hline Total & Both & $2.35 \pm_{0.66}^{1.13}$ \\
\hline
\end{tabular}

Table 8.3: Numbers of Cerenkov background events in the fiducial volume and above the energy threshold arising from the $\mathrm{U}$ and Th concentrations in the $\mathrm{AV}, \mathrm{D}_{2} \mathrm{O}$, and $\mathrm{H}_{2} \mathrm{O}$.

\begin{tabular}{|c|c|c|}
\hline Medium & Source & Number of Internal Bkg. Neutrons \\
\hline $\mathrm{D}_{2} \mathrm{O}$ & ${ }^{238} \mathrm{U}$ & $42 \pm_{10.6}^{7.0}$ \\
\hline $\mathrm{D}_{2} \mathrm{O}$ & ${ }^{232} \mathrm{Th}$ & $45 \pm 27$ \\
\hline \hline $\mathrm{D}_{2} \mathrm{O}$ & Total & $87 \pm_{29}^{27}$ \\
\hline
\end{tabular}

Table 8.4: Numbers of detected internal neutrons arising from the $\mathrm{U}$ and $\mathrm{Th}$ in the $\mathrm{D}_{2} \mathrm{O}$.

number of neutrino events.

In addition to the Čerenkov background from the $U$ and Th chain activity, we must also estimate the number of free neutrons produced by the photodisintegration of deuterium. The background neutrons from $\mathrm{U}$ and $\mathrm{Th}$ in the $\mathrm{D}_{2} \mathrm{O}$ will be distributed uniformly throughout the $\mathrm{D}_{2} \mathrm{O}$ and will look identical to the neutrons from the $\mathrm{NC}$ interactions. In [99] it was estimated that for the levels of $\mathrm{U}$ and $\mathrm{Th}$ present in the $\mathrm{D}_{2} \mathrm{O}, 0.293 \pm 0.18$ neutrons per day are produced in the $\mathrm{D}_{2} \mathrm{O}$ from $\mathrm{Th}$ in the $\mathrm{D}_{2} \mathrm{O}$, and $0.277 \pm_{0.070}^{0.046}$ neutrons per day are produced in the $\mathrm{D}_{2} \mathrm{O}$ from $\mathrm{U}$. In Chapter 6, it was found that $39.1 \%$ of neutrons will be captured and be detected within the signal region. The number of internal background neutrons from $\mathrm{U}$ and $\mathrm{Th}$ in the $\mathrm{D}_{2} \mathrm{O}$ for the 391.4 day dataset are summarized in Table 8.4. 
In addition to the background neutrons produced in the $\mathrm{D}_{2} \mathrm{O}$, neutrons will also be produced from $\mathrm{U}$ and $\mathrm{Th}$ activity in the $\mathrm{AV}$ and $\mathrm{H}_{2} \mathrm{O}$. However, all of these neutrons, coming from outside of the heavy water will have a different radial profile than the neutrons which are generated uniformly in the $\mathrm{D}_{2} \mathrm{O}$. Therefore, during the signal extraction process to be described in Chapter 9, the number of external neutron events is included as a parameter to be determined by the fit to the data, along with the numbers of events from the various neutrino signals.

\section{3 ${ }^{238} \mathrm{U}$ and ${ }^{232} \mathrm{Th}$ in the PMTs and PSUP}

Though the PMT components were specially selected to be as low in radioactivity as possible, they still contain a significant amount of radioactivity. Here the largest sources of radioactivity that can contribute are the PMT glass and the PMT reflector petals, since these are fully exposed to the rest of the detector. The U and Th content of the PMT glass has been estimated in [100] and upper limits one the $\mathrm{U}$ and Th content in the PMT reflector petals are presented in [53]. Only the front faces of the PMT dynodes and the ABS plastic buckets surrounding the PMTs are visible to the detector, so only a small amount of the light should enter the detector, with the rest being absorbed by the surrounding material. I have assumed that less than $50 \%$ of the mass of the PMT dynode and plastic buckets can

contribute to the radioactivity background seen in the detector. An upper limit of $5 \times 10^{-9}$ $\mathrm{g} / \mathrm{g}$ of $\mathrm{U}$ and $\mathrm{Th}$ in the plastic bucket has been obtained in measurements by LBNL and the content in the dynode is given in [100]. The concentrations and total mass of $\mathrm{U}$ and Th from these sources is summarized in Table 8.5. 


\begin{tabular}{|c|c|c|c|c|}
\hline Component & Source & $\begin{array}{c}\text { Amount per } \\
\text { PMT }(\mu \mathrm{g})\end{array}$ & Progeny & $\begin{array}{c}\text { Progeny } \\
\text { Rate }\left(\mathrm{s}^{-1}\right)\end{array}$ \\
\hline PMT Glass & ${ }^{238} \mathrm{U}$ & 18 & ${ }^{214} \mathrm{Bi}$ & 2100 \\
\hline PMT Glass & ${ }^{232} \mathrm{Th}$ & 18 & ${ }^{208} \mathrm{Tl}$ & 250 \\
\hline PMT Dynode & ${ }^{238} \mathrm{U}$ & 16.9 & ${ }^{214} \mathrm{Bi}$ & 1980 \\
\hline PMT Dynode & ${ }^{232} \mathrm{Th}$ & 16.1 & ${ }^{208} \mathrm{Tl}$ & 220 \\
\hline PMT Petals & ${ }^{238} \mathrm{U}$ & $<26.6$ & ${ }^{214} \mathrm{Bi}$ & $<3200$ \\
\hline PMT Petals & ${ }^{232} \mathrm{Th}$ & $<2.7$ & ${ }^{208} \mathrm{Tl}$ & $<40$ \\
\hline PMT Bucket & ${ }^{238} \mathrm{U}$ & $<7.2$ & ${ }^{214} \mathrm{Bi}$ & $<850$ \\
\hline PMT Bucket & ${ }^{232} \mathrm{Th}$ & $<7.2$ & ${ }^{208} \mathrm{Tl}$ & $<100$ \\
\hline \hline Total & ${ }^{238} \mathrm{U}$ & $<68.7$ & ${ }^{214} \mathrm{Bi}$ & $<8130$ \\
\hline Total & ${ }^{232} \mathrm{Th}$ & $<44$ & ${ }^{208} \mathrm{Tl}$ & $<610$ \\
\hline
\end{tabular}

Table 8.5: Concentration of $\mathrm{U}$ and Th in PMT components.

Unlike the $\mathrm{U}$ and $\mathrm{Th}$ in the $\mathrm{H}_{2} \mathrm{O}$ and $\mathrm{D}_{2} \mathrm{O}$, I will use a source instead of $\mathrm{MC}$ to estimate the number of Th and $\mathrm{U}$ background events that reconstruct inside of the fiducial volume and above the energy threshold. (The PMT backgrounds take an extremely long time to simulate due to the large number of events, and the detector optics are much more uncertain at large radii.) A Th source was placed into the detector near the PSUP, 849.8 $\mathrm{cm}$ from the center of the detector. The source was deployed for 1908.6 minutes, and is known to have $2.0 \pm 0.1 \times 10^{7}{ }^{208} \mathrm{Tl}$ decays per minute [101]. So, $3.81 \times 10^{10}{ }^{208} \mathrm{Tl}$ decays would have occurred during the source runs. In looking at runs where the source was not present, we would expect to see $16.23 \pm 0.23$ events above $5.5 \mathrm{MeV}$ and inside of $550 \mathrm{~cm}$ during the the source runs. However, in the Th source runs, $33 \pm 5$ (syst.) \pm 5.7 (stat.) events were observed inside the signal window, with an excess of $16.75 \pm 5$ (syst.) \pm 5.7 (stat.) events. (The systematic uncertainty includes a $\pm 1 \%$ uncertainty on the energy scale, a smearing of the energy resolution, and a $\pm 10 \mathrm{~cm}$ uncertainty on the fiducial volume cut.)

In 391 days of SNO neutrino data, we expect roughly $2.06 \times 10^{10}{ }^{208} \mathrm{Tl}$ decays to 


\begin{tabular}{|c|c|c|}
\hline Medium & Source & Number of Bkg Events \\
\hline PMTs & ${ }^{238} \mathrm{U}$ & $<11.5$ \\
\hline PMTs & ${ }^{232} \mathrm{Th}$ & $<13.1$ \\
\hline \hline PMTs & Combined & $<17.4$ \\
\hline
\end{tabular}

Table 8.6: Numbers of Cerenkov background events to the CC and ES signals arising from the $\mathrm{U}$ and $\mathrm{Th}$ in the PMTs.

occur in the PMTs (See Table 8.5). By comparing this to the number of events in the Th source data, we expect fewer than $9.0 \pm 2.7$ (syst.) \pm 3.1 (stat.) of these events to be inside the signal region.

For U, we expect $2.75 \times 10^{11}{ }^{214} \mathrm{Bi}$ decays to occur in the PMTs during the 391 day dataset. This is 13.3 times the rate of ${ }^{208} \mathrm{Tl}$ decays. However, from MC simulations of events at large radii, we expect that for the same number of ${ }^{214} \mathrm{Bi}$ and ${ }^{208} \mathrm{Tl}$ decays, we expect to only see $6.3 \pm 1.6 \%$ as many ${ }^{214} \mathrm{Bi}$ events (since the total energy in the decay is much less). Therefore, we can expect fewer than $7.58 \pm 3.0$ (syst.) \pm 2.6 (stat.) events to make it into the signal region of the dataset from the U content in the PMT components.

Since many of the U and Th estimates for the PMT components in Table 8.5 are upper limits, we should take both of these values as an upper limit, giving limits of $<13.1$ events from PMT Th and $<11.5$ events for PMT U. These are summarized in Table 8.6. We only need to be concerned the PMT radioactivity as a background to the Cerenkov signal. Any neutrons which might be coming from the PMT radioactivity, will be accounted for in the signal extraction process by the fit for the number of external neutrons. 


\subsection{External Gamma Rays}

In addition to the low-energy events arising directly from the $\mathrm{U}$ and $\mathrm{Th}$ within the detector components, there are also high-energy $\gamma$-ray produced outside the $\mathrm{D}_{2} \mathrm{O}$ region that can reconstruct within the neutrino signal region. High-energy $\gamma$-rays can originate in the rock, in the PMT glass and the PMT Support Structure (PSUP). The high-energy $\gamma$-rays which are emitted from the rock wall will preferentially appear near the equator of the SNO detector, due to the cylindrical shape of the SNO cavity. The flux of high-energy $\gamma$-rays coming from the PSUP and from the PMT glass will appear isotropically.

High-energy $\gamma$-rays can be produced by $(\mathrm{n}, \gamma)$ interactions on elements such as $\mathrm{Fe}$ in the rock walls and PSUP stainless steel. The fast neutrons in these reactions arise from the spontaneous fission of $\mathrm{U}$ and Th and from $(\alpha, \mathrm{n})$ reactions involving $\alpha$ s from the $\mathrm{U}$ and Th decay chains. Another major source of external $\gamma$-rays is $\alpha$-induced $\gamma$-rays, from $(\alpha, \gamma)$, $(\alpha, \mathrm{n} \gamma)$ and $(\alpha, \mathrm{p} \gamma)$ reactions on elements in the PMT glass such as $\mathrm{Al}, \mathrm{B}, \mathrm{Si}$, and $\mathrm{K}$ [102]. Again, these $\alpha$ s come from the Th and U decay chains. Of particular concern here are $\alpha$ reactions on aluminum which can produce $\gamma$-rays greater than $9 \mathrm{MeV}$.

In order to study the behavior of $\gamma$-rays in the SNO detector, we can utilize the ${ }^{16} \mathrm{~N}$ source, which provides a tagged source of $6.13 \mathrm{MeV} \gamma$-rays. This source was deployed in the guide tubes at large radii during the salt phase, at both positive and negative $\mathrm{z}$ positions. The ${ }^{16} \mathrm{~N}$ source data has been cleaned up by requiring the presence of an event tag in the ${ }^{16} \mathrm{~N}$ source and all of the standard data cleaning cuts have been applied except for the nhit burst cut. We can perform a box analysis, as depicted in Figure 8.4 using the ${ }^{16} \mathrm{~N}$ source data in a shell of the $\mathrm{H}_{2} \mathrm{O}$ to determine the high-energy background in the neutrino 
dataset. We can define a scaling factor for the source data,

$$
\eta\left(\text { Energy } \geq E_{f}, R_{f i t}<R_{f}\right)=\frac{\text { Number of Events in } \Omega_{f}}{\text { Number of Events in } \Omega_{0}}
$$

where $\Omega_{0}$ is defined as a radial shell in the $\mathrm{H}_{2} \mathrm{O}$ and $\Omega_{f}$ is the fiducial volume for neutrino analysis.

As the sources were located at $\mathrm{r}=800 \mathrm{~cm}$, it is desirable to choose a radial shell which is inside of $800 \mathrm{~cm}$ to insure that we are only looking at the part of the radial distribution that is falling smoothly and pointing inward. The radial profile of the ${ }^{16} \mathrm{~N}$ source was found to peak at roughly $775 \mathrm{~cm}$, so a radial slice of 725 to $775 \mathrm{~cm}$ was chosen for $\Omega_{0}$. It is also desirable to pick an energy threshold above the $\mathrm{U}$ and Th chain backgrounds in the $\mathrm{H}_{2} \mathrm{O}$. So, a lower bound of $6.50 \mathrm{MeV}$ and an upper bound of $14.5 \mathrm{MeV}$ (visible energy) were selected to define the $\Omega_{0}$ region in the light water. We would also like to restrict ourselves to events that are going inward. This is done using the parameter $\vec{u} \cdot \vec{r}$, which is the dot product of the unit vector describing the fit direction (u) and the unit vector describing the fit vertex $(r)$. Events which are pointing inwards toward the center of the detector will have $\vec{u}$ and $\vec{r}$ pointing in opposite directions, giving a $\vec{u} \cdot \vec{r}$ value of -1 . A cut of $\vec{u} \cdot \vec{r}<-0.75$ has also been chosen for $\Omega_{0}$. So, the final selection of $\Omega_{0}$ is

$$
\Omega_{0} \equiv 725<R_{f i t}<775,6.5 \leq \text { Energy }<14.5 \mathrm{MeV}, \vec{u} \cdot \vec{r}<-0.75
$$

The fiducial region for the neutrino analysis, $\Omega f$, is the same as that used for the neutrino analysis, namely

$$
\Omega_{f} \equiv 0<R_{\text {it }}<550 \mathrm{~cm}, 5.5 \mathrm{MeV} \leq \text { Energy } .
$$

With the two box regions, one can use the ${ }^{16} \mathrm{~N}$ data to obtain $\eta$. By looking at the number of events in the neutrino dataset in $\Omega_{0}$ and using the $\eta$ obtained from the ${ }^{16} \mathrm{~N}$ runs, 


\section{SNO Cavity}

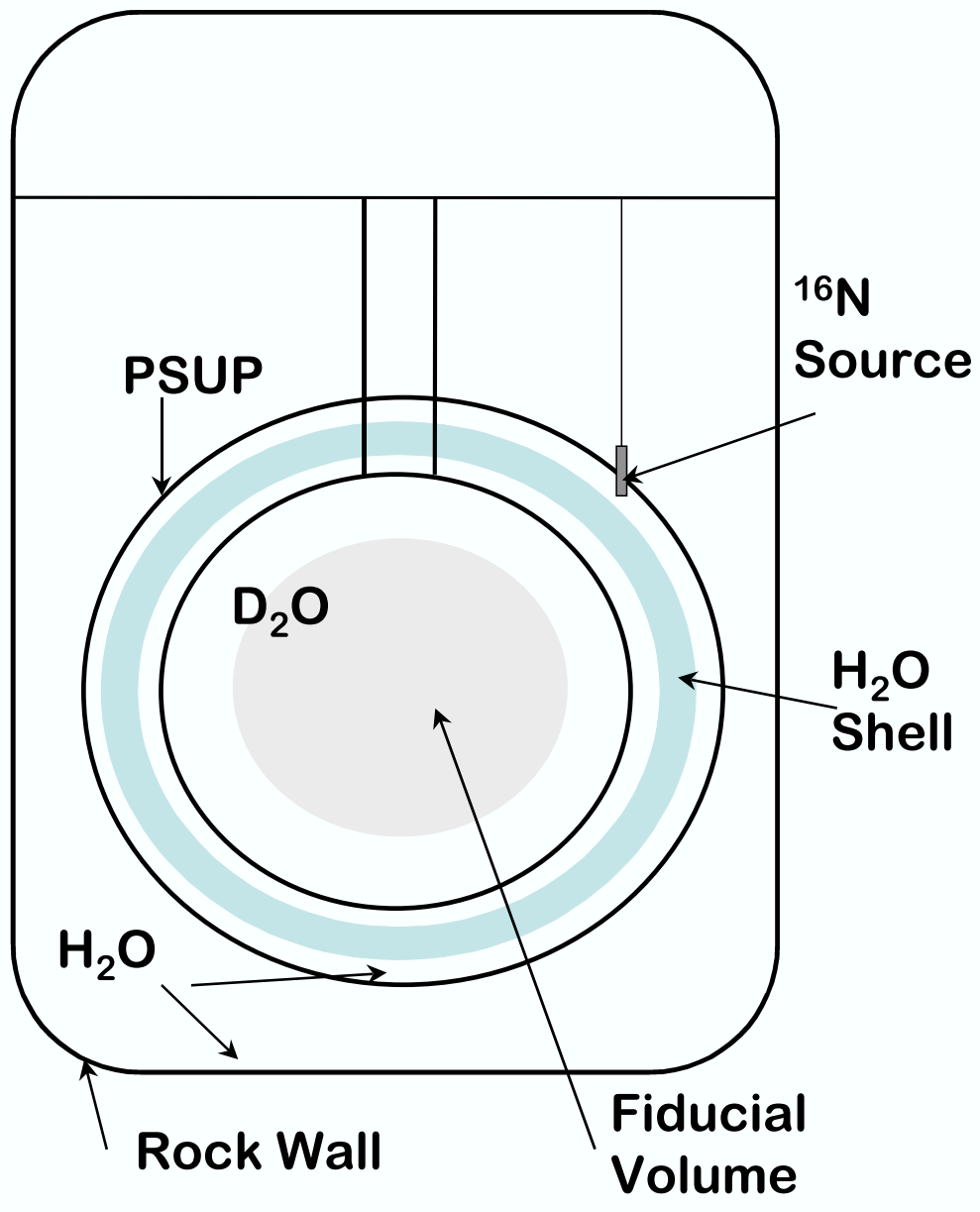

Figure 8.4: SNO cavity, the fiducial volume, and the high-radius shell used with the ${ }^{16} \mathrm{~N}$ source for estimating the high-energy $\gamma$-ray contamination. 
the number of high-energy $\gamma$-ray events in the signal region of the neutrino data, $\Omega_{f}$, can be inferred. It should be noted that this number represents the misreconstructed background for the Čerenkov signals and does not necessarily represent the physical number of $\gamma$-rays that enter the $\mathrm{D}_{2} \mathrm{O}$. Table 8.7 gives estimated high-energy background contamination inside $550 \mathrm{~cm}$ based on two ${ }^{16} \mathrm{~N}$ runs, one at $+\mathrm{z}$ and one and $-\mathrm{z}$. Taking the average of the upper and lower values yields a contamination of $6.32 \pm 1.41$ for $R_{f i t}<550 \mathrm{~cm}$, with the error here being statistical.

\begin{tabular}{|c|c|c|c|}
\hline \hline Source & $\mathrm{Z}_{\text {source }}$ & \# Events in Data & Stat. Uncert. \\
\hline \hline${ }^{16} \mathrm{~N}$ & +503 & 6.71 & \pm 2.25 \\
\hline${ }^{16} \mathrm{~N}$ & -503 & 5.93 & \pm 1.72 \\
\hline \hline${ }^{16} \mathrm{~N}$ & Average & 6.32 & \pm 1.41 \\
\hline \hline
\end{tabular}

Table 8.7: Estimated external $\gamma$-ray contamination in the neutrino data with $R_{f i t}<550$ $\mathrm{cm}$, energy $>5.50 \mathrm{MeV}$. The errors are statistical only.

We must also consider some of the systematic uncertainties on this estimate, summarized in Table 8.8. As was noted above, there appears to be a slight asymmetry between the runs at the top and the run at the bottom of the detector, which gives an uncertainty of $5.3 \%$. We can also consider what happens when the cut values on $\Omega_{0}$ are relaxed or tightened. The $\vec{u} \cdot \vec{r}$ cut value was varied to -0.8 and -0.5 . The radial cut on $\Omega_{0}$ was broadened to $675<R_{\text {fit }}<775 \mathrm{~cm}$. Also the low-energy bound on $\Omega_{0}$ was changed from $6.5 \mathrm{MeV}$ to $6.0 \mathrm{MeV}$ and to $7.0 \mathrm{MeV}$. From $\mathrm{MC}$ simulations of $\mathrm{U}$ and $\mathrm{Th}$ in the $\mathrm{H}_{2} \mathrm{O}$ described in section 8.2, less than 1 event from the $\mathrm{H}_{2} \mathrm{O} \mathrm{U}$ and Th backgrounds is expected to enter into $\Omega_{0}$. These systematics are listed in Table 8.8. Combining the statistical and systematic uncertainties gives an estimate of $6.32 \pm_{1.6}^{2.1}$ events, or a $68 \%$ CL limit of $<8.42$. 


\begin{tabular}{|c|c|}
\hline \hline Systematic & Uncertainty \\
\hline \hline Z-Asymmetry & $\pm 6.2 \%$ \\
\hline Varying $\vec{u} \cdot \vec{r}$ cut & $\pm_{2.5}^{1.7 \%}$ \\
\hline Varying R cut & $\pm 7.1 \%$ \\
\hline Varying E cut & $\pm_{9.2}^{23.7} \%$ \\
\hline $\mathrm{H}_{2} \mathrm{O}$ U and Th & $<0.08 \%$ \\
\hline \hline Total & $\pm_{11.4}^{25.6 \%}$ \\
\hline \hline
\end{tabular}

Table 8.8: Systematics on estimates of external $\gamma$-rays.

\subsection{Muon-Induced Spallation}

Another source of backgrounds to the SNO experiment are cosmic-ray muons. Due to its great depth (6800 ft or 6000 m.w.e.), the muon flux in SNO is less than 100 per day. The muons themselves are quite distinctive due to the large amount of energy that they deposit (roughly $5 \mathrm{MeV}$ per $\mathrm{cm}[18]$ ) both inside the detector and into the light water veto outside the PMTs. Light produced in the light water outside the PSUP is detected by the outward-looking tubes, and serves as a muon veto. The muons themselves are not a significant background for neutrino analysis. However, when a muon travels through the SNO detector, several processes can occur which can leave behind long-lived radioactive nuclei. The first such process is muon capture, where a muon stops in the detector, captures on a nucleus, and a muon neutrino is produced. An example of such a process is $\mu+{ }_{8}^{16} \mathrm{O} \rightarrow{ }_{7}^{16} \mathrm{~N}+\nu_{\mu}$.

Another process which can occur is muon-induced spallation, where a muon breaks apart a nucleus into smaller fragments, some of which can be radioactive. These unstable products can be backgrounds to the NC solar neutrino signal if they decay by the production of a neutron, or if they produce a $\gamma$-ray with an energy above $2.2 \mathrm{MeV}$, which can 
photodisintegrate ${ }^{2} \mathrm{H}$. The radioactive muon followers can also form a background to the CC signal if they undergo beta decay. Table 8.9 lists all of the possible spallation and muoncapture products that can be produced by nuclei up to ${ }^{37} \mathrm{Cl}$ that have half-lives longer than 1 second and decay by the emission of a neutron, a $\beta$ above $4 \mathrm{MeV}$, or a $\gamma$-ray above 2.2 $\mathrm{MeV}$.

The muons can also disintegrate ${ }^{2} \mathrm{H}$, producing prompt neutrons. The majority of the neutrons will thermalize and capture on ${ }^{35} \mathrm{Cl}$ in the salt $\mathrm{D}_{2} \mathrm{O}$ in roughly 5 msec and should be removed by the muon follower cut. However, the neutrons produced by muons will be much higher in energy than those produced by the NC interaction. From [103], the neutron energies can be over $100 \mathrm{MeV}$. So, we might also be concerned with these fast neutrons undergoing $(\mathrm{n}, \mathrm{p})$ and $(\mathrm{n}, \alpha)$ reactions to produce long lived nuclei.

\subsubsection{Spallation on ${ }^{16} \mathrm{O}$}

From muon spallation on ${ }^{16} \mathrm{O}$ there are several nuclei with half-lives longer than 5 seconds that might still remain in the data after the $20 \mathrm{sec}$ muon follower short cut is applied. To get an idea of the spallation production rate, we can look at Super-K data. Based on the Super-K thesis of Ishino [104], roughly 1,444,000 events above $2 \mathrm{MeV}$ (total energy) were removed by the spallation cut in 503.8 days of data. Assuming that these events were all real spallation events, and using the fact that the muon rate in Super-K is roughly $2 \mathrm{~Hz}$ for the entire inner detector, we can estimate that there are roughly $2866{ }^{16} \mathrm{O}$ spallation events per day from 172,800 muons per day, for a spallation fraction of roughly 1.65\%. Recall that Super-K does not see the neutrons produced by spallation, so we can use this number as an estimate of the fraction of the time radioactive nuclei are produced 


\begin{tabular}{|c|c|c|c|c|c|c|}
\hline Nuc. & $\begin{array}{l}\text { Mode } \\
\text { Decay }\end{array}$ & $\begin{array}{l}(\%) \\
\text { B.R. }\end{array}$ & $\begin{array}{c}(\mathrm{MeV}) \\
\beta \text { Max }\end{array}$ & $\begin{array}{c}(\mathrm{MeV}) \\
\gamma \text { Max }\end{array}$ & $\begin{array}{l}\text { Product } \\
\text { Unstable }\end{array}$ & $\begin{array}{r}\text { (sec) } \\
\mathbf{T}_{1 / 2}\end{array}$ \\
\hline${ }_{4}^{11} \mathrm{Be}$ & $\beta^{-} \gamma$ & 86.1 & 11.5 & $<2.2$ & - & 13.81 \\
\hline${ }_{4}^{11} \mathrm{Be}$ & $\beta^{-} \gamma$ & 10.7 & 6.5 & $>2.2$ & - & 13.81 \\
\hline${ }_{6}^{15} \mathrm{C}$ & $\beta^{-}$ & 37 & 9.771 & - & - & 2.5 \\
\hline${ }_{6}^{15} \mathrm{C}$ & $\beta^{-} \gamma$ & 63 & 4.47 & 5.3 & - & 2.5 \\
\hline${ }_{7}^{16} \mathrm{~N}$ & $\beta^{-}$ & 28 & 10.42 & - & - & 7.13 \\
\hline${ }_{7}^{16} \mathrm{~N}$ & $\beta^{-} \gamma$ & 72 & 4.2 & $\geq 6.13$ & - & 7.13 \\
\hline${ }_{7}^{17} \mathrm{~N}$ & $\beta^{-} n$ & 95 & $\sim 3$ & - & - & 4.17 \\
\hline${ }_{7}^{17} \mathrm{~N}$ & $\beta^{-} \gamma$ & 5 & 8.6 & 0.870 & - & 4.17 \\
\hline${ }_{8}^{14} \mathrm{O}$ & $\beta^{+} \gamma$ & 99.3 & 2.831 & 2.312 & - & 70.6 \\
\hline${ }_{8}^{19} \mathrm{O}$ & $\beta^{-} \gamma$ & 99.9 & 4.8 & $<2.2$ & - & 26.9 \\
\hline${ }_{8}^{21} \mathrm{O}$ & $\beta^{-} \gamma$ & $\sim 35$ & 4.65 & $>2.2$ & ${ }_{9}^{21} \mathrm{~F}$ & 3.4 \\
\hline${ }_{8}^{21} \mathrm{O}$ & $\beta^{-} \gamma$ & $\sim 65$ & 6.739 & $<2.2$ & ${ }_{9}^{21} \mathrm{~F}$ & 3.4 \\
\hline${ }_{9}^{20} \mathrm{~F}$ & $\beta^{-} \gamma$ & 100.0 & 5.391 & 1.633 & - & 11.00 \\
\hline${ }_{9}^{21} \mathrm{~F}$ & $\beta^{-} \gamma$ & 99.8 & 5.684 & $<2.2$ & - & 4.16 \\
\hline${ }_{9}^{22} \mathrm{~F}$ & $\beta^{-} \gamma$ & $\sim 28$ & 5.295 & $>2.2$ & - & 4.23 \\
\hline${ }_{9}^{22} \mathrm{~F}$ & $\beta^{-} \gamma$ & $\sim 72$ & 9.54 & $<2.2$ & - & 4.23 \\
\hline${ }_{9}^{23} \mathrm{~F}$ & $\beta^{-} \gamma$ & $\sim 25$ & 6.165 & $>2.2$ & - & 2.23 \\
\hline${ }_{9}^{23} \mathrm{~F}$ & $\beta^{-} \gamma$ & $\sim 75$ & 8.48 & $<2.2$ & - & 2.23 \\
\hline${ }_{10}^{18} \mathrm{Ne}$ & $\beta^{+} \gamma$ & 100 & 4.446 & 1.04 & ${ }_{9}^{18} \mathrm{~F}$ & 1.67 \\
\hline${ }_{10}^{23} \mathrm{Ne}$ & $\beta^{-} \gamma$ & 100 & 4.37 & $<2.2$ & - & 37.2 \\
\hline${ }_{10}^{* 24} \mathrm{Ne}$ & $\beta^{-} \gamma$ & 100 & 1.998 & 0.874 & ${ }_{11}^{24} \mathrm{Na}$ & 202.8 \\
\hline $\begin{array}{l}24 \\
{ }_{11}^{24} \mathrm{Na}\end{array}$ & $\beta^{-} \gamma$ & 100 & 1.393 & 2.75 & - & 53820 \\
\hline${ }_{11}^{26} \mathrm{Na}$ & $\beta^{-} \gamma$ & $\sim 95$ & 7.504 & $<2.2$ & - & 1.072 \\
\hline${ }_{11}^{26} \mathrm{Na}$ & $\beta^{-} \gamma$ & $\sim 5$ & 6.37 & $>2.2$ & - & 1.072 \\
\hline $\begin{array}{l}22 \\
12\end{array}$ & $\beta^{+} \gamma$ & 100 & 4.2 & 1.9 & $\begin{array}{l}22 \\
11\end{array}$ & 3.857 \\
\hline $\begin{array}{l}23 \\
12\end{array}$ & $\beta^{+} \gamma$ & 100 & 4.056 & 0.439 & - & 11.32 \\
\hline $\begin{array}{l}29 \\
12 \mathrm{Mg}\end{array}$ & $\beta^{-} \gamma$ & 65.8 & 5.32 & $>2.2$ & $\begin{array}{l}29 \\
13\end{array}$ & 1.3 \\
\hline${ }_{12}^{29} \mathrm{Mg}$ & $\beta^{-} \gamma$ & 34.2 & 7.55 & $<2.2$ & $\begin{array}{l}29 \\
13\end{array}$ & 1.3 \\
\hline $\begin{array}{l}24 \\
13\end{array}$ & $\beta^{+} \gamma$ & 100 & 9.756 & $>2.7$ & - & 2.07 \\
\hline $\begin{array}{l}25 \\
13\end{array}$ & $\beta^{+}$ & 99.1 & 4.277 & - & - & 7.183 \\
\hline${ }_{13}^{26} \mathrm{Al}(\mathrm{GS})$ & $\beta^{+} \gamma$ & 2.7 & 1.066 & 2.94 & - & $2 \times 10^{13}$ \\
\hline${ }_{13}^{26} \mathrm{Al}(0+)$ & $\beta^{+}$ & 100 & 4.232 & 2.12 & - & 6.345 \\
\hline
\end{tabular}

Table continues ....

Table 8.9: All of the possible muon-induced spallation or muon-capture products that have half-lives greater than 1 second and decay by the production of a neutron or a beta with an energy greater than $4 \mathrm{MeV}$ or a $\gamma$-ray with an energy above $2.2 \mathrm{MeV}$ [60]. 
Table 8.9 continued ....

\begin{tabular}{|l|c|c|r|r|c|r|}
\hline \hline Nuc. & $\begin{array}{c}\text { Mode } \\
\text { Decay }\end{array}$ & $\begin{array}{c}\text { (\%) } \\
\text { B.R. }\end{array}$ & $\begin{array}{c}\text { (MeV) } \\
\beta \text { Max }\end{array}$ & $\begin{array}{c}\text { (MeV) } \\
\gamma \text { Max }\end{array}$ & $\begin{array}{c}\text { Product } \\
\text { Unstable }\end{array}$ & $\begin{array}{c}(\text { sec) } \\
\mathbf{T}_{1 / 2}\end{array}$ \\
\hline${ }_{13}^{29} \mathrm{Al}$ & $\beta^{-} \gamma$ & 63 & 1.2 & 2.42 & - & 393.6 \\
\hline${ }_{13}^{30} \mathrm{Al}$ & $\beta^{-} \gamma$ & 100 & 6.326 & $>2.23$ & - & 3.68 \\
\hline${ }_{14}^{26} \mathrm{Si}$ & $\beta^{+} \gamma$ & 100 & 4.838 & 1.850 & ${ }_{13}^{26} \mathrm{Al}(0+)$ & 2.234 \\
\hline${ }_{14}^{27} \mathrm{Si}$ & $\beta^{+}$ & 99.7 & 4.811 & - & - & 4.16 \\
\hline${ }_{14}^{33} \mathrm{Si}$ & $\beta^{-} \gamma$ & $\sim 9$ & 3.307 & 2.538 & ${ }_{15}^{33} \mathrm{P}$ & 6.18 \\
\hline${ }_{14}^{29} \mathrm{P}$ & $\beta^{+}$ & 98.3 & 4.943 & - & - & 4.14 \\
\hline${ }_{15}^{30} \mathrm{P}$ & $\beta^{+}$ & 99.9 & 4.232 & - & - & 150 \\
\hline${ }_{15}^{34} \mathrm{P}$ & $\beta^{-} \gamma$ & 99.9 & 5.374 & $<2.2$ & - & 12.43 \\
\hline${ }_{15}^{30} \mathrm{~S}$ & $\beta^{+} \gamma$ & 2.3 & 3.119 & 3.019 & ${ }_{15}^{30} \mathrm{P}$ & 1.178 \\
\hline${ }_{16}^{30} \mathrm{~S}$ & $\beta^{+} \gamma$ & 97.7 & 6.138 & 0.709 & ${ }_{15} \mathrm{P}$ & 1.178 \\
\hline${ }_{16}^{31} \mathrm{~S}$ & $\beta^{+}$ & 98.9 & 5.396 & - & - & 2.57 \\
\hline${ }_{16}^{37} \mathrm{~S}$ & $\beta^{-} \gamma$ & 94 & 1.76 & 3.103 & - & 303 \\
\hline${ }_{16}^{37} \mathrm{~S}$ & $\beta^{-}$ & 5.6 & 4.865 & - & - & 303 \\
\hline${ }_{16}^{33} \mathrm{Cl}$ & $\beta^{+}$ & 98.6 & 5.582 & - & - & 2.511 \\
\hline${ }_{17}^{33} \mathrm{Cl}$ & $\beta^{+}$ & 100 & 5.492 & - & - & 1.52 \\
\hline \hline
\end{tabular}

by muon-induced spallation on ${ }^{16} \mathrm{O}$.

The average muon path length in SNO is much shorter than in Super-K. Super$\mathrm{K}$ is roughly $40 \mathrm{~m}$ in diameter while $\mathrm{SNO}$ is only $18 \mathrm{~m}$ in diameter. Assuming that the spallation probability is proportional to the muon pathlength, the spallation fraction in SNO is reduced by $55 \%$. The average muon energy in SNO is roughly $339 \mathrm{GeV}$, while the average muon-energy in Super-K (at 2700 m.w.e.) is $293 \mathrm{GeV}$ (See Equation 11 in [105]). The muon-spallation cross section scales as $E^{0.75}$ [105], so we can estimate that the spallation fraction in SNO on ${ }^{16} \mathrm{O}$ is roughly $0.83 \%$. Using a muon flux of $70 /$ day, this will translate into roughly 0.58 spallation products/day. In looking at Table 8.9, only ${ }_{4}^{11}$ Be with its 13.83 sec half-life will have a significant contribution after the $20 \mathrm{sec}$ muon follower cut. But in the Appendix of Koshio's Super-K thesis [106], ${ }_{4}^{11}$ Be made up only about $5 \times 10^{-3 \%}$ of the observed spallation products. Therefore the contribution from long-lived ${ }_{8}^{16} \mathrm{O}$ spallation 
products should be less than 0.011 events for 390 days of data, which is negligible.

\subsubsection{Spallation on $\mathrm{Cl}$ and $\mathrm{Na}$}

The spallation rate for ${ }^{16} \mathrm{O}$ is roughly $0.83 \%$ per muon. However, there are far fewer $\mathrm{Na}$ and $\mathrm{Cl}$ atoms in the detector than $\mathrm{O}$ atoms, so we must scale the interaction rates accordingly. In a $0.2 \% \mathrm{NaCl}$ brine, for every $1000{ }^{16} \mathrm{O}$ atoms, there are roughly $0.674{ }^{23} \mathrm{Na}$ atoms, $0.512{ }^{35} \mathrm{Cl}$ atoms, and $0.162{ }^{37} \mathrm{Cl}$ atoms. In [107], it is shown that the average cross section for exciting a compound nucleus by the absorption of electric dipole radiation scales as NZ/A. So, for ${ }^{16} \mathrm{O},{ }^{24} \mathrm{Na},{ }^{35} \mathrm{Cl}$, and ${ }^{37} \mathrm{Cl}$ respectively this factor is $4,5.7,8.7$ and 9.2 .

Using the appropriate scale factors given above and the fact that 33 muons per day travel through the salted heavy water, only about $2.6 \times 10^{-4}$ spallation products per

day will come from ${ }^{23} \mathrm{Na}, 3.0 \times 10^{-4}$ will come from ${ }^{35} \mathrm{Cl}$, and $1.0 \times 10^{-4}$ will come from ${ }^{37} \mathrm{Cl}$. Even if every one of these decay products were long lived and produced a high-energy $\gamma$-ray capable of photodisintegration, the background to the neutrino signal would be 0.26 events for 391 days of data, which is not significant.

\subsubsection{Spallation Neutrons}

Though a large number of neutrons are likely to be produced by muons (estimated to be 0.44 per muon in [108]), the neutrons will capture with a lifetime of $5 \mathrm{msec}$, and these will be eliminated from the data set via the $20 \mathrm{sec}$ muon follower cut (See Chapter 7). In the event that a muon is not tagged by the muon veto, then an additional cut, the "missed muon follower cut", removes all events which are within $250 \mathrm{msec}$ of an event with more than 150 hits. If the muon instead passed through the cavity, but did not enter the PSUP, 


\begin{tabular}{|l|c|c|}
\hline \hline Nucleus & $\sigma$ (barns) & Unstable Product \\
\hline \hline${ }^{1} \mathrm{H}$ & 0.3326 & - \\
\hline${ }^{2} \mathrm{H}$ & 0.000519 & ${ }^{3} \mathrm{H}$ \\
\hline${ }^{12} \mathrm{C}$ & 0.00353 & - \\
\hline${ }^{13} \mathrm{C}$ & 0.00137 & ${ }^{14} \mathrm{C}$ \\
\hline${ }^{16} \mathrm{O}$ & 0.000190 & - \\
\hline${ }^{17} \mathrm{O}$ & 0.00054 & - \\
\hline${ }^{18} \mathrm{O}$ & 0.00016 & ${ }^{19} \mathrm{O}$ \\
\hline${ }^{23} \mathrm{Na}$ & 0.530 & ${ }^{24} \mathrm{Na}$ \\
\hline${ }^{35} \mathrm{Cl}$ & 43.6 & - \\
\hline${ }^{37} \mathrm{Cl}$ & 0.433 & ${ }^{38} \mathrm{Cl}$ \\
\hline \hline
\end{tabular}

Table 8.10: Thermal neutron-capture cross sections from [60] for the nuclei found in the heavy water, AV, and light water of SNO.

then this could potentially also be a source of neutrons in the detector. A study was done to look for events where light was detected in the OWL tubes but less than 150 hits were seen in the detector [109]. One of these events had a follower after it in the data. A limit of $\leq 1$ event has been assigned for this background.

\subsubsection{Delayed Neutron-Capture Reactions}

We must also consider neutron-capture reactions that can produce long lived radionuclides. Table 8.10 lists the neutron-capture cross sections for the nuclei which can be found in the SNO light water, AV, and salted heavy water. Though the vast majority of neutrons will capture on ${ }^{35} \mathrm{Cl}$, a small number will produce ${ }^{24} \mathrm{Na}$ (half-life of 14.96 hours) and ${ }^{38} \mathrm{Cl}$ (half-life of 37.2 minutes). ${ }^{38} \mathrm{Cl}$ only produces a $\gamma$-ray with an energy above 2.2 $\mathrm{MeV}$ in $0.02 \%$ of the decays, so it does not represent a serious photodisintegration background. With beta energies up to $4.9 \mathrm{MeV}$, it could pose a background for the CC and ES signal. 
A MC study of 20,000 neutrons distributed uniformly throughout the salted $\mathrm{D}_{2} \mathrm{O}$ and the $\mathrm{AV}$ neck gave $78.6 \%$ of the neutrons capturing on natural $\mathrm{Cl}$ and $1.1 \%$ of the events capturing on ${ }^{23} \mathrm{Na}$. Unfortunately SNOMAN does not directly simulate neutron capture on ${ }^{37} \mathrm{Cl}$, but by considering the relative cross sections and concentrations of ${ }^{23} \mathrm{Na}$ and ${ }^{37} \mathrm{Cl}$ (the natural abundance of ${ }^{37} \mathrm{Cl}$ is $24.2 \%$ ), we can estimate that roughly $0.22 \%$ of neutrons capture on ${ }^{37} \mathrm{Cl}$. Q.R. Ahmad performed a study of muon followers in the SNO detector [108] in which he concluded that the total neutron production rate following a muon is roughly $0.44 \pm 0.02$ neutrons per muon, averaged over all muons. Then for 70 muons/day we might expect $0.068{ }^{38} \mathrm{Cl}$ atoms and $0.34{ }^{24} \mathrm{Na}$ atoms to be produced per day. Based on a MC study of ${ }^{38} \mathrm{Cl}$ decays, only $0.157 \pm 0.023 \%$ had an energy above $5.50 \mathrm{MeV}$ and reconstructed inside $550 \mathrm{~cm}$. Therefore, the amount of muon-produced ${ }^{38} \mathrm{Cl}$ seen in the data should not be significant ( 0.048 events for 391 days of data). For ${ }^{24} \mathrm{Na}$, only $0.0051 \%$ of the decays will have a measured energy above $5.50 \mathrm{MeV}$ and reconstruct inside of $550 \mathrm{~cm}$, but nearly every decay produces a $2.75 \mathrm{MeV} \gamma$-ray. Roughly 1 in $3802.75 \mathrm{MeV} \gamma$-rays will

photodisintegrate deuterium [110]. Therefore, ${ }^{24} \mathrm{Na}$ produced following a muon will yield a neutral-current background of roughly $8.9 \times 10^{-4}$ neutrons per day, (0.35 neutrons for 391 days of data) and a Čerenkov backgrounds of $1.7 \times 10^{-5}$ events per day (or 0.007 events in 391 days of data).

\subsection{5 (n,p) Reactions and Muon Capture}

If high-energy neutrons are produced by muons, it might also be possible for (n,p) reactions to occur. Muons that stop in the detector can decay or capture on a nucleus, producing the same products as $(\mathrm{n}, \mathrm{p})$ reactions. Table 8.11 gives all of the possible products 
of $(n, p)$ or muon-capture reactions in the SNO salted water. The three nuclei which could pose the most serious background are ${ }^{16} \mathrm{~N},{ }^{23} \mathrm{Ne}$, which has betas up to $4.375 \mathrm{MeV}$, and ${ }^{37} \mathrm{~S}$, which could pose quite a troublesome background with its $3.1 \mathrm{MeV} \gamma$-rays and 5 minute half-life.

It is difficult to estimate the production rates, since they depend on the neutron energy spectrum resulting from spallation, which is uncertain. Instead we can look for the presence of ${ }^{16} \mathrm{~N}$ in the data following muons to obtain a limit. A study looking for events which are 1-50 seconds after a muon has been performed by Noah Oblath. No significant sign of a 7.13 second half-life was observed. An upper limit of 0.77 events in the 391-day dataset (after the $20 \mathrm{sec}$ muon follower cut has been applied) has been reported [111].

The cross sections for $(\mathrm{n}, \mathrm{p})$ interactions on ${ }^{23} \mathrm{Na}[112]$ and ${ }^{37} \mathrm{Cl}[113]$ are roughly the same magnitude or smaller than the ${ }^{16} \mathrm{O}(\mathrm{n}, \mathrm{p})$ cross section [114]. Since the concentrations of $\mathrm{Na}$ and $\mathrm{Cl}$ are so much smaller, the backgrounds will be negligible.

As for muon capture, 1-2\% of muons stop in the detector [64], for a total of 0.7 to 1.4 events per day in SNO. In the Kamiokande detector, approximately $42 \%$ of the muons were found to be $\mu^{-}[115]$. Assuming that at SNO's depth the ratio will remain the same, there should be at most 0.58 stopped $\mu^{-}$per day in the SNO detector. According to [116], the muon-capture rate in $\mathrm{H}_{2} \mathrm{O}$ is $1.02 \times 10^{5} \mathrm{~s}^{-1}$ compared to a muon decay rate of $4.55 \times 10^{5}$ $\mathrm{s}^{-1}$ in vacuum. In the presence of natural $\mathrm{Cl}$, the muon-capture rate is $1.64 \times 10^{6} \mathrm{~s}^{-1}$, while it is $0.337 \times 10^{6} \mathrm{~s}^{-1}$ for $\mathrm{Na}$. Taking in to account the fact that $\mathrm{SNO}$ is only $0.2 \% \mathrm{NaCl}$, yields a total $\mu^{-}$interaction rate of $5.58 \times 10^{5} \mathrm{~s}^{-1}$, of which approximately $18.3 \%$ will be nuclear capture on ${ }^{16} \mathrm{O}, 0.6 \%$ will be nuclear capture on $\mathrm{Cl}$ (with roughly $24 \%$ of these on 
${ }^{37} \mathrm{Cl}$ ), $0.1 \%$ will be muon capture on $\mathrm{Na}$, and the remainder will be muon decay. Therefore, only 0.11 muons per day will capture on ${ }^{16} \mathrm{O}$. However, there are only 4 neutron bound states of ${ }^{16} \mathrm{~N}$. It has been found that $1.66 \%$ of stopped muons in water form bound states of ${ }^{16} \mathrm{~N}$ [117], with the remainder emitting a neutron and forming ${ }^{15} \mathrm{~N}$, which is stable. So, this reduces the amount of ${ }^{16} \mathrm{~N}$ from stopped muons which will undergo $\beta \gamma$ decay to 0.0097 per day. The fiducial volume cut should also have an effect here. These ${ }^{16} \mathrm{~N}$ nuclei will be distributed throughout the light and heavy water. Assuming that this distribution is uniform, then roughly $35 \%$ of the ${ }^{16} \mathrm{~N}$ decays will occur in the $\mathrm{D}_{2} \mathrm{O}$, for a total of $0.0034 \beta \gamma$ events per day. Assuming a perfect efficiency for the muon tag for stopped muons, then a 20 s muon follower cut will reduce this number to $0.0005{ }^{16} \mathrm{~N}$ decays per day. In reality, the muon tag probably does not have a perfect efficiency for tagging these low-energy muons, so an upper limit of $0.0034{ }^{16} \mathrm{~N}$ events/day will be used here. There should be approximately 0.0008 muon captures per day on ${ }^{37} \mathrm{Cl}$ and 0.0006 captures per day on ${ }^{23} \mathrm{Na}$. For a 391 day dataset this gives an estimate of 0.31 and 0.23 captures on ${ }^{37} \mathrm{Cl}$ and ${ }^{24} \mathrm{Na}$ respectively.

\subsubsection{Summary of Muon-Induced Backgrounds}

Table 8.12 summarizes the muon-induced backgrounds that remain in the fiducial volume, above the energy threshold, after the 20 sec muon follower cut has been applied. 


\begin{tabular}{|l|c|c|r|r|c|r|}
\hline \hline $\begin{array}{l}\text { Target } \\
\text { Nucleus }\end{array}$ & $\begin{array}{c}\text { Product } \\
\text { Nucleus }\end{array}$ & Mode & $\begin{array}{r}\text { B.R. } \\
(\mathbf{\%})\end{array}$ & $\begin{array}{c}\text { Max } \beta \mathbf{E} \\
(\mathbf{M e V})\end{array}$ & $\begin{array}{c}\text { Max } \gamma \mathbf{E} \\
(\mathbf{M e V})\end{array}$ & $\begin{array}{r}\mathbf{T}_{1 / 2} \\
(\mathbf{s e c})\end{array}$ \\
\hline \hline${ }_{6}^{12} \mathrm{C}$ & ${ }_{5}^{12} \mathrm{~B}$ & $\beta^{-}$ & 97.2 & 13.37 & - & 0.020 \\
\hline${ }_{6}^{13} \mathrm{C}$ & ${ }_{5}^{13} \mathrm{~B}$ & $\beta^{-} \gamma$ & 100 & 13.44 & 3.68 & 0.0174 \\
\hline${ }_{8}^{16} \mathrm{O}$ & ${ }_{7}^{16} \mathrm{~N}$ & $\beta^{-}$ & 28 & 10.42 & - & 7.13 \\
\hline${ }_{8}^{16} \mathrm{O}$ & ${ }_{7}^{16} \mathrm{~N}$ & $\beta^{-} \gamma$ & 72 & 4.2 & $\geq 6.13$ & 7.13 \\
\hline${ }_{8}^{17} \mathrm{O}$ & ${ }_{7}^{17} \mathrm{~N}$ & $\beta^{-} \mathrm{n}$ & 95 & $\sim 3$ & - & 4.17 \\
\hline${ }_{8}^{17} \mathrm{O}$ & ${ }_{7}^{17} \mathrm{~N}$ & $\beta^{-} \gamma$ & 5 & 8.68 & 0.870 & 4.17 \\
\hline${ }_{8}^{18} \mathrm{O}$ & ${ }_{7}^{18} \mathrm{~N}$ & $\beta^{-} \gamma$ & 40 & 11.92 & $>2.2$ & 0.624 \\
\hline${ }_{11}^{23} \mathrm{Na}$ & ${ }_{10}^{23} \mathrm{Ne}$ & $\beta^{-} \gamma$ & 100 & 4.375 & $<2.2$ & 37.2 \\
\hline${ }_{17}^{35} \mathrm{Cl}$ & ${ }_{16}^{35} \mathrm{~S}$ & $\beta^{-}$ & 100 & 0.167 & - & $7.5 \times 10^{6}$ \\
\hline${ }_{17}^{37} \mathrm{Cl}$ & ${ }_{16}^{37} \mathrm{~S}$ & $\beta^{-} \gamma$ & 94 & 1.762 & 3.10 & 303 \\
\hline \hline
\end{tabular}

Table 8.11: The common (n,p) or muon-capture reactions which can occur in the SNO detector. The half-lives and other characteristics of the product nucleus are given in [60].

\begin{tabular}{|c|c|c|c|}
\hline \hline & \multirow{2}{*}{ Process } & Target & \multicolumn{2}{|c|}{ Events in 391 days } \\
\cline { 3 - 4 } & Cerenkov & $\mathrm{n}$ \\
\hline Spallation & ${ }^{16} \mathrm{O}$ & 0.11 & 0 \\
\hline Spallation & ${ }^{23} \mathrm{Na}$ & 0.10 & 0 \\
\hline Spallation & $\mathrm{Cl}$ & 0.16 & 0 \\
\hline Spallation $\mathrm{n}$ & - & - & $\leq 1$ \\
\hline Spallation $\mathrm{n}$ cap. & ${ }^{23} \mathrm{Na}$ & $<0.007$ & 0.35 \\
\hline Spallation $\mathrm{n}$ cap. & ${ }^{37} \mathrm{Cl}$ & $<0.048$ & 0 \\
\hline (n,p) & ${ }^{16} \mathrm{O}$ & $<0.77$ & 0 \\
\hline (n,p) & ${ }^{23} \mathrm{Na}$ & $\sim 0$ & 0 \\
\hline (n,p) & ${ }^{37} \mathrm{Cl}$ & $\sim 0$ & 0 \\
\hline$\mu^{-}$capture & ${ }^{16} \mathrm{O}$ & $<1.32$ & 0 \\
\hline$\mu^{-}$capture & ${ }^{23} \mathrm{Na}$ & $<0.23$ & 0 \\
\hline$\mu^{-}$capture & ${ }^{17} \mathrm{Cl}$ & $<0.31$ & 0 \\
\hline \hline Total & $\mathrm{All}$ & $<2.1$ & $<1.35$ \\
\hline \hline
\end{tabular}

Table 8.12: The muon-induced backgrounds which remain in the data after the 20 sec muon follower cut, the $R_{f i t}<550 \mathrm{~cm}$ cuts, and the energy $>5.50 \mathrm{MeV}$ cut. 


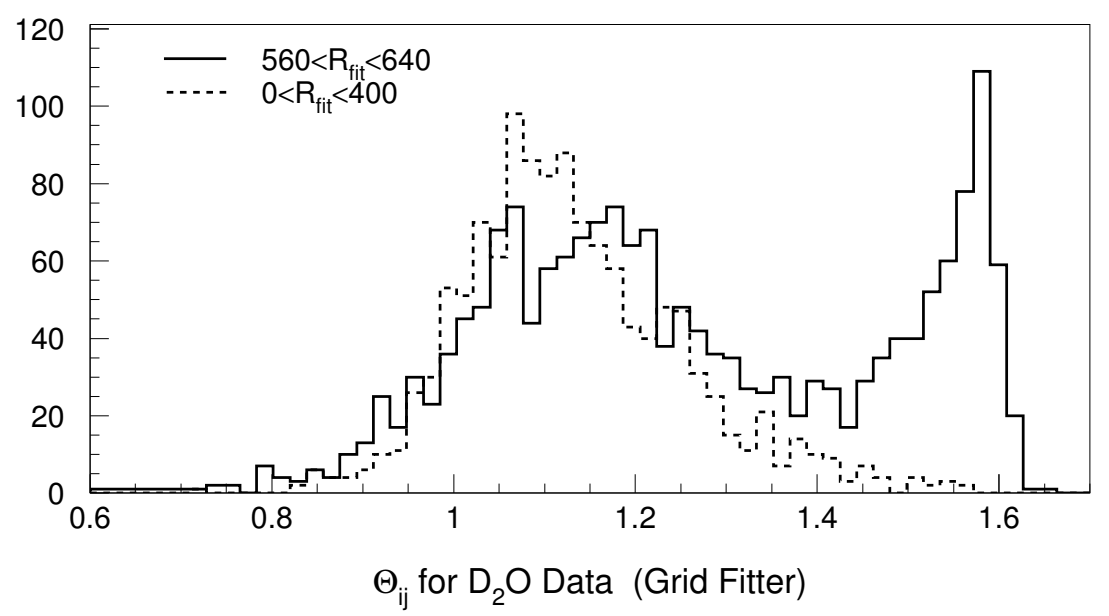

Figure 8.5: $\theta_{i j}$ for data from the pure $\mathrm{D}_{2} \mathrm{O}$ phase with the Level 1 instrumental backgrounds cuts applied. A larger $\theta_{i j}$ value indicates a more isotropic event. Notice the excess of events above $\theta_{i j}$ of 1.45 for $\mathrm{R}$ near $600 \mathrm{~cm}$.

\subsection{Acrylic Vessel Events}

\subsubsection{Description of the Events}

Another class of background events, characterized by a reconstructed position near the AV and a highly isotropic hit pattern, has been observed in the data. As can be seen in the pure $\mathrm{D}_{2} \mathrm{O}$ data in Figure 8.5, there is an excess of isotropic events in the range of $560<\mathrm{R}<640$. Applying the In-Time Ratio (ITR) cut (See Chapter 7) can remove some, but not all of these events (See Figure 8.6). In this dissertation this class of events will be referred to as "AV" events.

As can be seen in Figures 8.7, 8.8, and 8.9, the "AV" events appear to reconstruct all over the AV, though perhaps more so in the upper hemisphere. A few distinct clusters are visible, some of which are located near the joints between AV panels. However, a study 


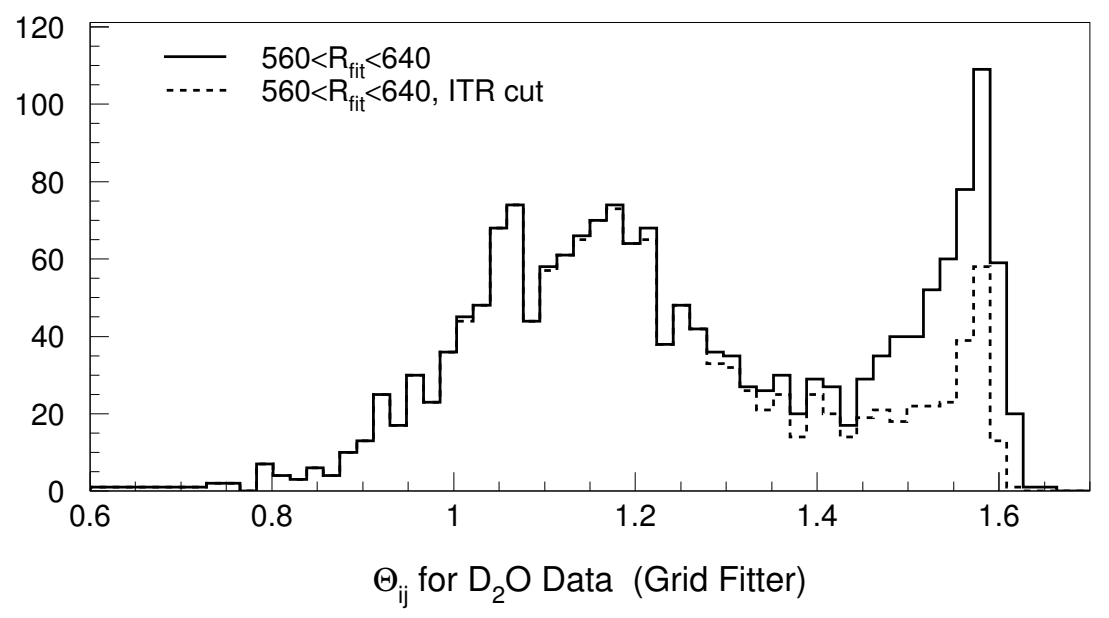

Figure 8.6: $\theta_{i j}$ for data from the pure $\mathrm{D}_{2} \mathrm{O}$ phase with the Level 1 instrumental backgrounds cuts applied. Notice that when the ITR cut is applied, many of the highly isotropic events are removed.

described in [95], did not observed a significant difference in the $\theta_{i j}$ distribution for events which reconstructed near or far from a panel joint.

These highly isotropic events near the AV do not appear to occur at a particular time of day, as can be seen in Figure 8.10. Nor do they appear to change in rate over time (See Figure 8.11). From Figure 8.11, we can estimate the frequency of "AV" events to be less than 1 event per day above $4.5 \mathrm{MeV}$ (visible energy).

The exact origin of these events is unknown. They might be caused by bubbles nucleating on the surface of the AV or some sort of static charge build-up on the AV. Another possible explanation might be scintillation light coming from bubbles in the AV bonds, which would account for the highly isotropic nature of the light. The AV was cured in an argon atmosphere, so the bubbles might have argon in them (and possibly some nitrogen 


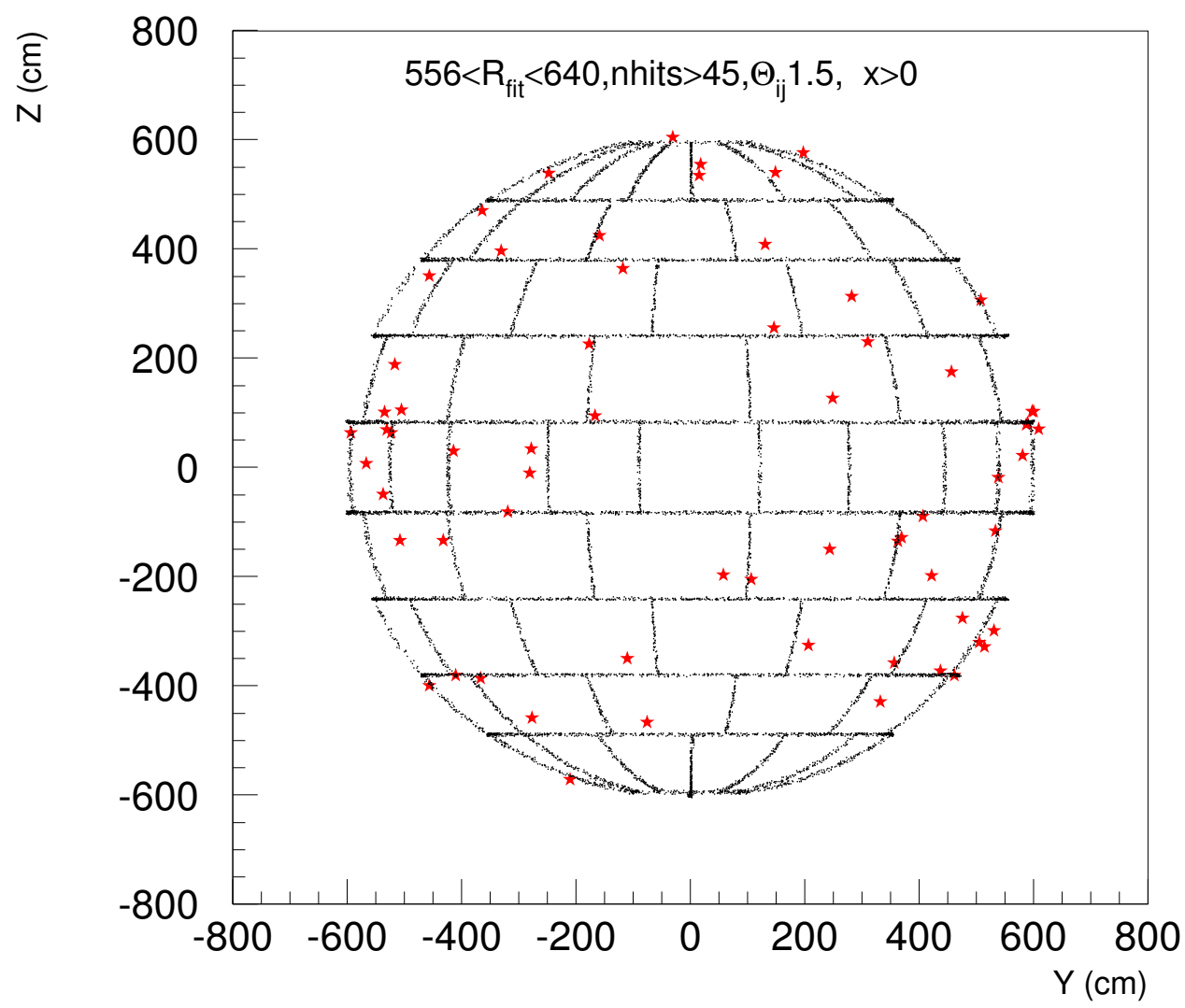

Figure 8.7: Shown here (as stars) are the reconstructed positions of events in the salt data with $\theta_{i j}$ greater than 1.45, a fit radius between 550 and $650 \mathrm{~cm}$, and nhits greater than or equal to 45. They have been superimposed over lines showing the locations of the AV tiles. 


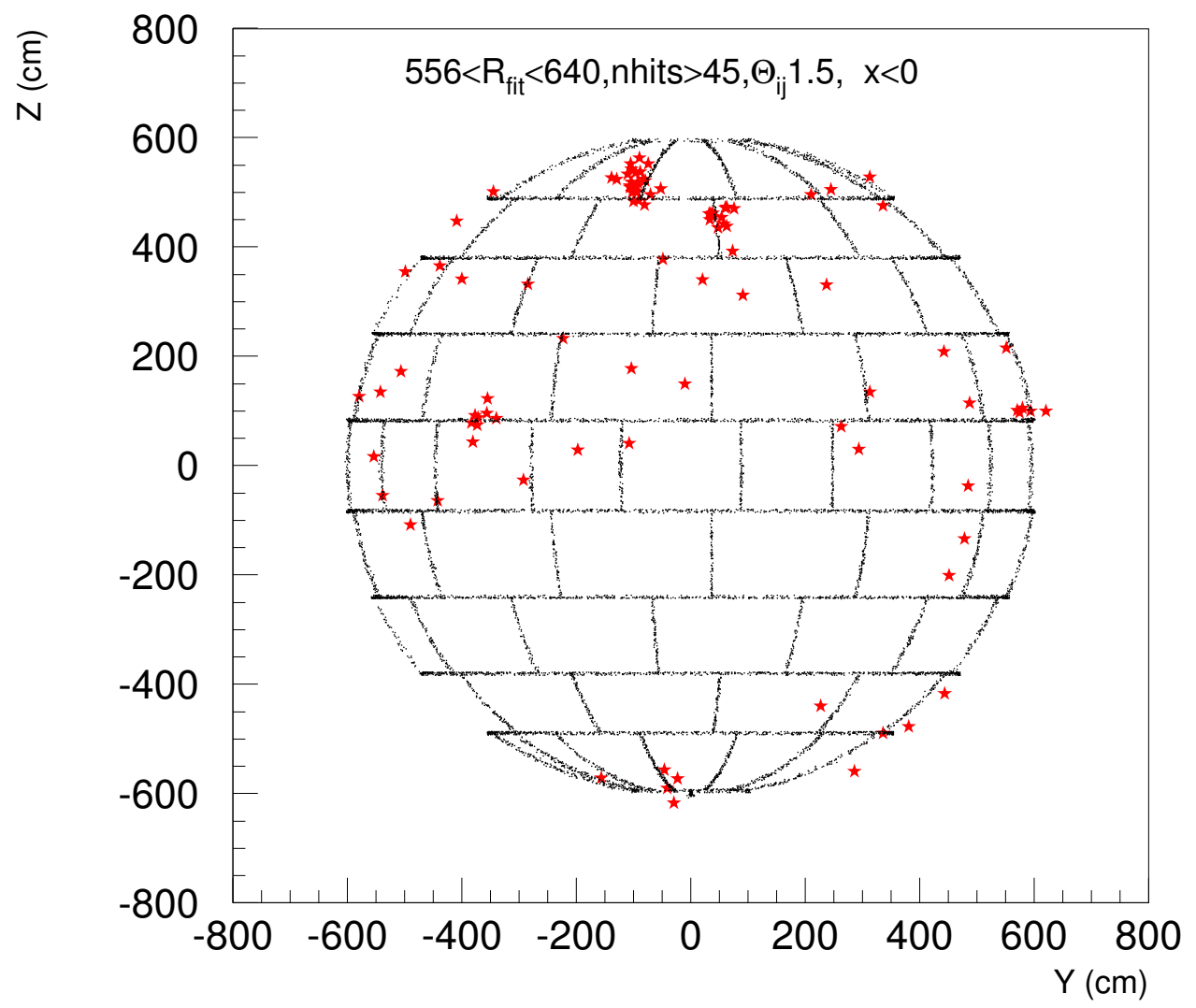

Figure 8.8: A side view of the locations of candidate AV events. 


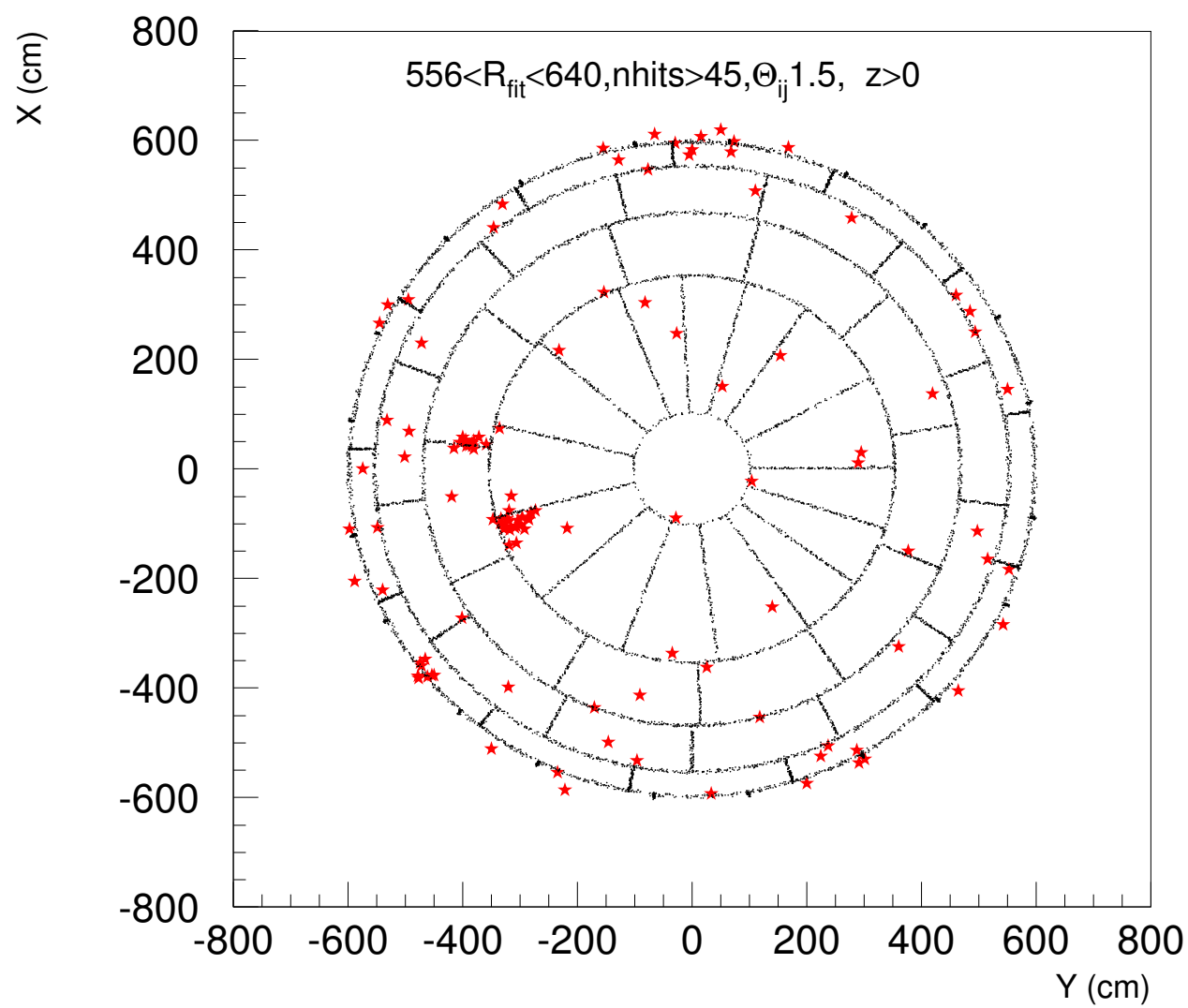

Figure 8.9: The reconstructed positions of events in the salt data with $\theta_{i j}$ greater than 1.45, a fit radius between 550 and $650 \mathrm{~cm}$, and nhits greater than or equal to 45 . 
from the mine air). However, as was estimated in [95], scintillation in air or argon bubbles can only explain these events if the bubbles are quite large (on the order of $0.5 \mathrm{~cm}$ ). Such bubbles would certainly have been noticed during the QA/QC of the acrylic vessel, and no visible bubbles were reported in the bonds. So, this is unlikely to explain their origin.

Another possibility is that the light is generated within the acrylic itself. Acrylic is known to emit light when it is fractured, through a process known as fractoluminescence. The MS thesis of Tom Andersen [118] focuses on the light emitted by acrylic when fractured or stressed. While large amounts of light were found in association with the fracture of acrylic, no light was consistently observed due to the stressing of the acrylic. However, the SNO detector houses a much larger volume of acrylic, which has been observed with far greater PMT coverage, for a much longer period of time. So, the previous tests may not have been sensitive enough to see light at the level required to explain the "AV" events. The theory of low-level acrylic stress might also explain why these events sometimes cluster near panel joints.

\subsubsection{Estimate of AV Event contamination}

During the pure $\mathrm{D}_{2} \mathrm{O}$ analysis the $\mathrm{AV}$ events were removed by placing an upper bound on $\theta_{i j}$; Events with $\theta_{i j}$ greater than 1.45 were removed. In the salt phase, this cut is problematic. Firstly, due to the more isotropic nature of neutron events in salt, this cut will remove between $7-10 \%$ of the neutron events [95]. Secondly, and perhaps more problematically, the cut removes a region of parameter space that contains much more of the neutron pdf than the CC or ES pdfs. Applying this cut is likely to result in an increase

in the overall correlation between the $\mathrm{NC}$ and $\mathrm{CC}$ signals. Without a cut on $\theta_{i j}$, the fiducial 


\section{Energy $>=4.5 \mathrm{MeV}$}

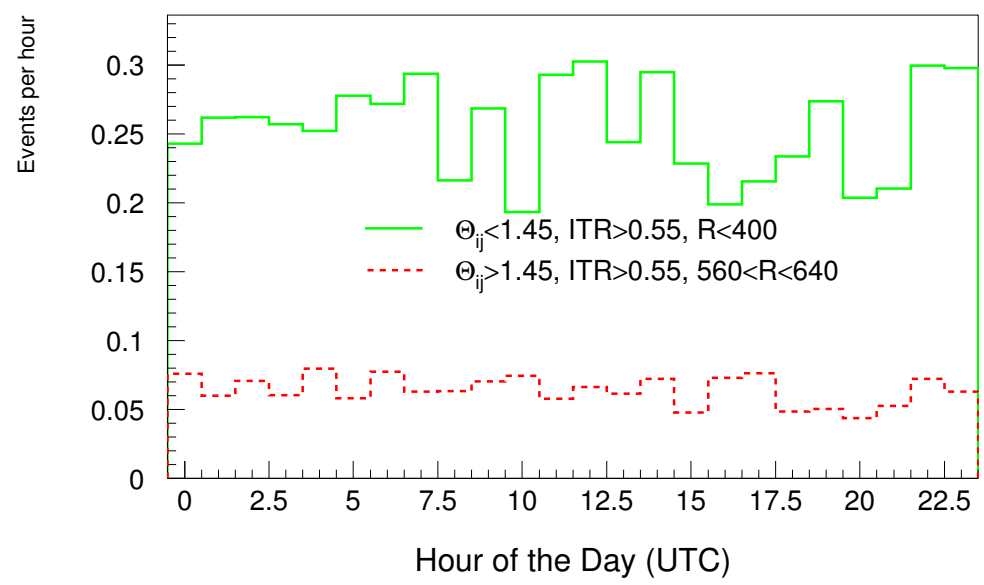

Figure 8.10: The dotted line shows the hourly rates for possible "AV" events in the salt data. The solid line shows the profile for events with a $\theta_{i j}<1.45$ and $R_{f i t}<400 \mathrm{~cm}$.

Daily Rates using Energy $>=4.5 \mathrm{MeV}$

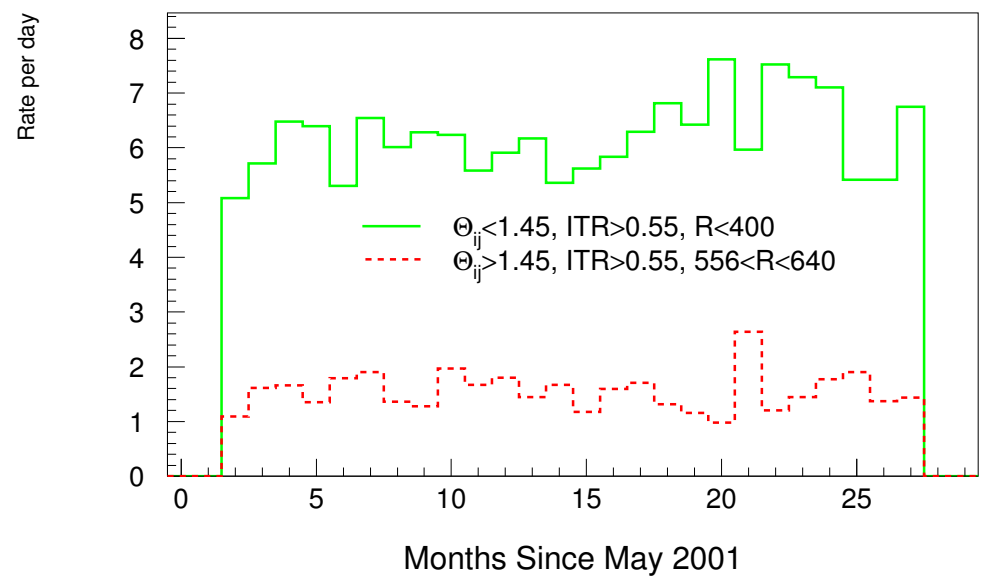

Figure 8.11: The dotted line shows the daily rates as a function of time for possible "AV" events in the salt data. The solid line shows the rates for events with $\theta_{i j}<1.45$, and $\mathrm{r}_{f i t}<400$ $\mathrm{cm}$, which are likely to be neutrino events and low-energy $\mathrm{D}_{2} \mathrm{O}$ backgrounds. 
volume cut of $R<550 \mathrm{~cm}$ should still remove the majority of these events. It is necessary to estimate this background.

The method used here involved constructing $\theta_{i j}$ probability distribution functions for neutrons and electrons. These pdfs were then fit to the clean neutrino data, and their normalizations were taken to be the number of electrons and neutrons in the data set. PDFs for the ${ }^{208} \mathrm{Tl}$ and ${ }^{214} \mathrm{Bi}$ background in the $\mathrm{D}_{2} \mathrm{O} A V, \mathrm{H}_{2} \mathrm{O}$ were included in the fit at a fixed normalization (See Section 8.2). The fit was only performed from $\theta_{i j}$ of 0.9 to 1.35 to avoid the influence of the AV events at large $\theta_{i j}$ values on the fit. An example of such fit is shown in Figure 8.12. Any discrepancy between the pdfs and the real data at high $\theta_{i j}$ was attributed to the AV events. The "AV" event contamination was then estimated by taking the difference between the number of events in the data above $\theta_{i j}$ of 1.5 and the number of events in the fit pdfs above 1.5 .

Table 8.13 shows the results of this technique for a variety of energy thresholds and fiducial volume cuts. For the fiducial volume of interest $\left(\mathrm{E}>5.50 \mathrm{MeV}, R_{\text {fit }}<550\right.$ $\mathrm{cm}$ ), the contamination is $-1.85 \pm 11.33$ (stat.) events. The fit to the data is shown in Figure 8.12. Compare this to the fit for $R_{f i t}<600 \mathrm{~cm}$, shown in Figure 8.13 where a statistically significant excess is seen at large $\theta_{i j}$. Here the uncertainty includes both the statistical uncertainty on the number of events in the dataset and the uncertainty due to the fit values for the electron and neutron pdf normalizations (taking into account the covariance between the fit values).

Some of the systematic studies that have been performed for $\mathrm{r}<550 \mathrm{~cm}$ and $\mathrm{E}>5.50$ $\mathrm{MeV}$ are shown in Table 8.14. As mentioned above, for these fits only the CC $\theta_{i j}$ pdf was 


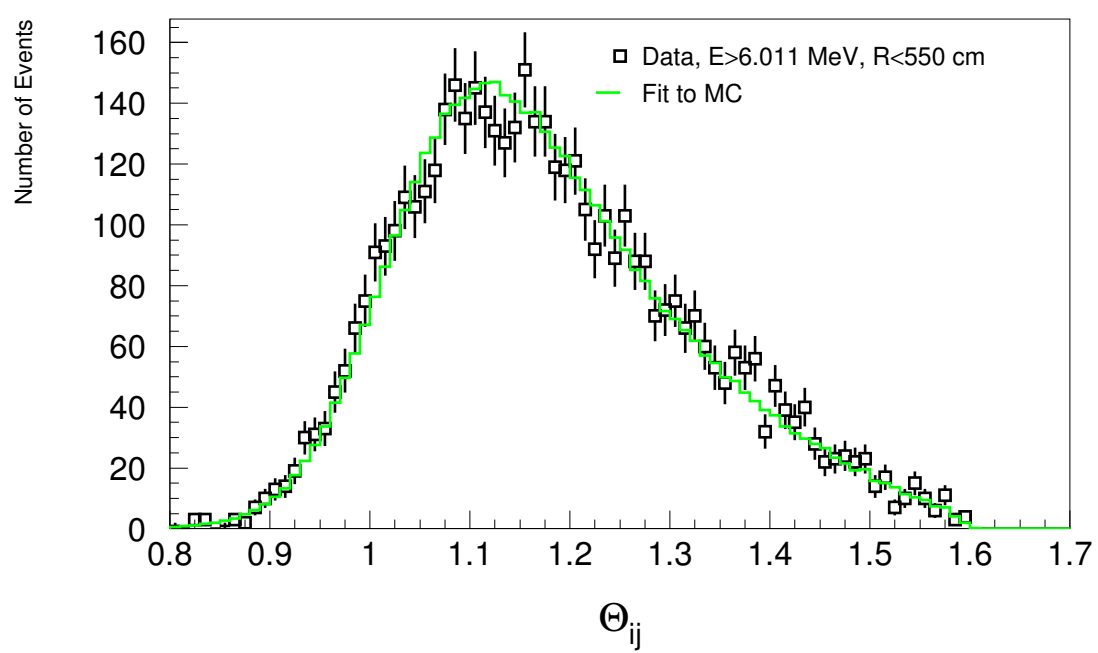

Figure 8.12: The $\theta_{i j}$ distribution for the salt data and the combined fit to the electron and neutron pdfs for all events which reconstruct inside $550 \mathrm{~cm}$ and above an energy cut of 5.50 $\mathrm{MeV}$.

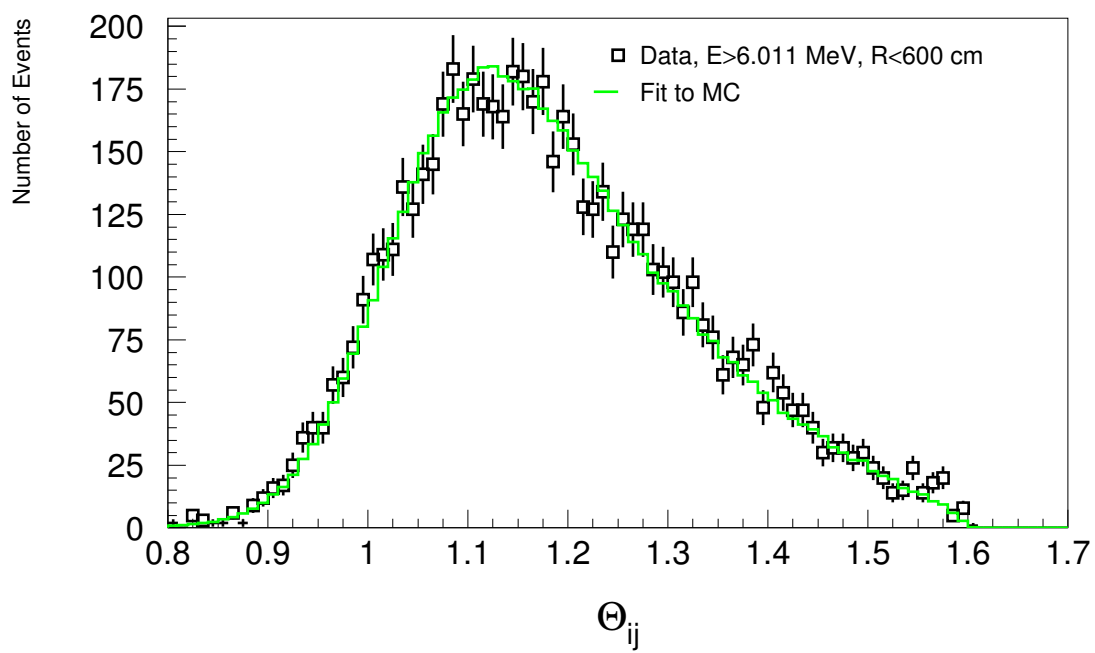

Figure 8.13: The $\theta_{i j}$ distribution for the salt data and the combined fit to the electron and neutron pdfs for all events which reconstruct inside $600 \mathrm{~cm}$ and above an energy cut of 5.50 $\mathrm{MeV}$. 


\begin{tabular}{|c|c|c|c|}
\hline R cut $(\mathrm{cm})$ & Energy $>(\mathrm{MeV})$ & Excess Events in Data & Stat Error \\
\hline \hline 525 & 5.50 & -1.4 & 10.6 \\
550 & 5.50 & -1.85 & 11.33 \\
575 & 5.50 & 0.74 & 12.40 \\
590 & 5.50 & 3.82 & 13.1 \\
600 & 5.50 & 25.2 & 14.3 \\
620 & 5.50 & 77.5 & 16.6 \\
\hline \hline
\end{tabular}

Table 8.13: Excess events in data above $\theta_{i j}$ of 1.5 .

\begin{tabular}{|c|c|}
\hline Systematic Study & Change on Fit Value (\# of Evs) \\
\hline \hline ES instead of CC & 0.45 \\
$0.5 \%$ shift in $\theta_{i j}$ & $\pm_{3.21}^{3.19}$ \\
Fit from $0.85<\theta_{i j}<1.4$ & -3.3 \\
Fit from $0.95<\theta_{i j}<1.3$ & +2.83 \\
\hline Total Systematic Uncert. & $\pm_{4.60}^{4.29}$ \\
\hline \hline
\end{tabular}

Table 8.14: Systematic studies of excess events in data above $\theta_{i j}$ of 1.5 for visible energy $>5.50 \mathrm{MeV}$ and Rfit $<550 \mathrm{~cm}$.

used to estimate the number of electron events in the data. In reality the electron signal will be composed of CC and a small amount of ES. So the fits were also performed using the ES pdf instead. The fits to the $\theta_{i j}$ profile were typically performed from 0.9 to 1.35 . The effects of broadening and narrowing the window are shown in Table 8.14. This technique is quite sensitive to errors on the $\theta_{i j}$ shape. To estimate the effect on the results of the uncertainty on the $\theta_{i j}$ shape, the signal MC pdfs were scaled up and down by $0.5 \%$. Taking all of the systematic errors in the table and adding them in quadrature gives an systematic uncertainty of $\pm_{4.60}^{4.29}$ events. Adding this in quadrature with the statistical uncertainty, gives an upper limit of 10.3 events. The technique described here only estimates the excess number of events above $\theta_{i j}=1.5$. In a MC simulation of isotropic light from the AV (with a Gaussian time spread of 3 nsec), of the 105 events which reconstructed inside $550 \mathrm{~cm}$ and 
above $5.50 \mathrm{MeV}, 17$ had a $\theta_{i j}$ below 1.5. Therefore, the contamination estimate here must be scaled up by $119 \%$. So, the upper limit on the "AV" event contamination estimate for $\mathrm{E}>5.50 \mathrm{MeV}$ and $\mathrm{R}_{\text {fit }}<550 \mathrm{~cm}$ is 12.3 events.

\subsection{Other Backgrounds}

In addition to the numerous sources of backgrounds already given in this chapter, there are still more physical processes that can contribute and have been evaluated in [111]. These backgrounds are summarized in Table 8.15 and I will briefly describe them below.

Fission: While ${ }^{238} \mathrm{U}$ usually follows the decay chain shown in Figure 8.2, it does

have a branching ratio of $5.45 \times 10^{-7}$ for spontaneous fission, which can produce both neutrons and $\gamma$-rays. An estimate of $1.2 \pm 0.2 \gamma$-ray events and $12.3 \pm 2.7$ neutron events was obtained in [111].

$\alpha$-induced neutrons: In addition to the $\gamma$-rays, which can break apart deuterons, an alpha with more than $6.66 \mathrm{MeV}$ can also disintegrate deuterium. Here only the $6.9 \mathrm{MeV}$ $\alpha$ from ${ }^{222} \mathrm{Po}$ in the Th chain is a concern. It has been estimated that Th chain activity and ${ }^{222} \mathrm{Rn}$ will produce approximately $3.73 \pm 0.73$ detected neutrons in the dataset from alpha disintegration of $\mathrm{D}$ [111]. There will also be $(\alpha, \mathrm{n})$ interactions that can occur on ${ }^{17} \mathrm{O}$ and ${ }^{18} \mathrm{O}$. Here the $\alpha$ s are coming from both the $\mathrm{U}$ and Th decay chain progenies. Using the stopping power for $\alpha \mathrm{s}$ in the various elements, an estimate of $2.63 \pm 0.44$ detected neutrons was obtained in $[109,111]$.

${ }^{24} \mathrm{Na}$ : Another concern are backgrounds from ${ }^{24} \mathrm{Na}$. Neutrons will capture on ${ }^{23} \mathrm{Na}$, producing ${ }^{24} \mathrm{Na}$, which has a 15 hour half-life and always produces a $2.75 \mathrm{MeV} \gamma$-ray. 


\begin{tabular}{|c|c|c|}
\hline \hline & \multicolumn{2}{|c|}{ Events in 391 days } \\
\cline { 2 - 3 } Process & Cerenkov & $\mathrm{n}$ \\
\hline Fission & $1.2 \pm 1.5$ & $12.3 \pm 2.7$ \\
\hline${ }^{2} \mathrm{H}(\alpha, \alpha \mathrm{n})^{1} \mathrm{H}$ & - & $3.73 \pm 0.73$ \\
\hline${ }^{17,18} \mathrm{O}(\alpha, \mathrm{n})^{20,21} \mathrm{Ne}$ & - & $2.63 \pm 0.44$ \\
\hline${ }^{24} \mathrm{Na}$ activation & - & $10.84 \pm 2.56$ \\
\hline Reactor and Terrestrial $\bar{\nu}$ & - & $2.51 \pm 0.30$ \\
\hline Atmos. $\nu$ & $7.26 \pm 2.18$ & $16.95 \pm 5.08$ \\
\hline n from CNO neutrinos & - & $0.43 \pm 0.43$ \\
\hline \hline Total & $8.5 \pm 2.2$ & $49.4 \pm 6.38$ \\
\hline \hline
\end{tabular}

Table 8.15: Summary of backgrounds from miscellaneous physical processes in the detector.

Therefore, this will be a photodisintegration neutron background. ${ }^{24} \mathrm{Na}$ production will be enhanced in the neck and during water recirculation where there is less shielding from the neutrons. ${ }^{24} \mathrm{Na}$ will also be produced during calibrations, so data is usually excluded from solar neutrino analysis for a period of time after a source is removed from the detector. The estimated number of neutrons that remain in the dataset due to ${ }^{24} \mathrm{Na}$ production in the pipes, neck, and calibration runs is $10.84 \pm 2.56$ [119].

CNO neutrinos: There are also some neutrino related backgrounds. While the primary source of solar neutrinos in SNO is the ${ }^{8} \mathrm{~B}$ branch of the p-p interaction chain, a small number of neutrinos from the CNO cycle are also present. The estimated number of observed neutrino interactions from these neutrinos is $0.43 \pm 0.43$ events for a 391 day dataset [111] for the SSM CNO fluxes.

$\bar{\nu} \mathbf{s}$ and Atmospheric $\nu$ s: The estimated background from anti-neutrinos (coming from reactors and other terrestrial sources) is $2.51 \pm 0.30$ neutrons. Atmospheric neutrinos are also estimated to contribute $16.95 \pm 5.08$ neutron events and $7.26 \pm 2.18 \gamma$-ray events above threshold [111]. 


\begin{tabular}{|c|c|c|c|}
\hline \hline \multirow{2}{*}{ Process } & \multicolumn{2}{|c|}{ Events in 391 days } & \\
\cline { 2 - 3 } & Cerenkov & Intern. $\mathrm{n}$ & Section \\
\hline $\mathrm{U}$ and Th in $\mathrm{D}_{2} \mathrm{O}, \mathrm{H}_{2} \mathrm{O}$, and AV & $2.35 \pm_{0.66}^{1.13}$ & $87 \pm_{29}^{27}$ & 8.2 \\
\hline $\mathrm{U}$ and Th in PMTs & $<17.4$ & - & 8.3 \\
\hline External $\gamma$-rays & $<8.42$ & - & 8.4 \\
\hline Muon-induced events & $<2.1$ & $<1.35$ & 8.5 \\
\hline "AV" Events & $<12.3$ & - & 8.6 \\
\hline Other Backgrounds & $8.5 \pm 2.2$ & $49.4 \pm 6.38$ & 8.7 \\
\hline \hline
\end{tabular}

Table 8.16: Summary all of the backgrounds described in this chapter that remain in the dataset following the application of the $R_{\text {fit }}<550 \mathrm{~cm}$ cut and the energy $>5.50 \mathrm{MeV}$ cut.

\subsection{Summary of Backgrounds}

Table 8.16 summarizes the estimates for all of the backgrounds discussed in this chapter. 


\section{Chapter 9}

\section{Signal Extraction}

\subsection{Introduction}

Up until this point, this dissertation has focussed on calibration of the detector response and estimation of the number of background events. We can now turn our attention to the extraction of the number of neutrino events in the data. As was discussed in Chapter 2, SNO can detect neutrinos via three interactions (CC, ES, and NC). It is not possible to distinguish these neutrino interactions from one another on an event by event basis. However, we can look at a large sample of these events and deduce how many events of each type were present.

There are some key differences between the various neutrino interactions which allow us to measure how many neutrino interactions of each type are present in the dataset. The charged-current and elastic-scattering interactions produce electrons, which give off a cone of Črenkov light, while the neutral-current interaction produces a neutron, which yields a more isotropic burst of $\gamma$-rays. The elastic-scattering reaction occurs in both $\mathrm{H}_{2} \mathrm{O}$ 


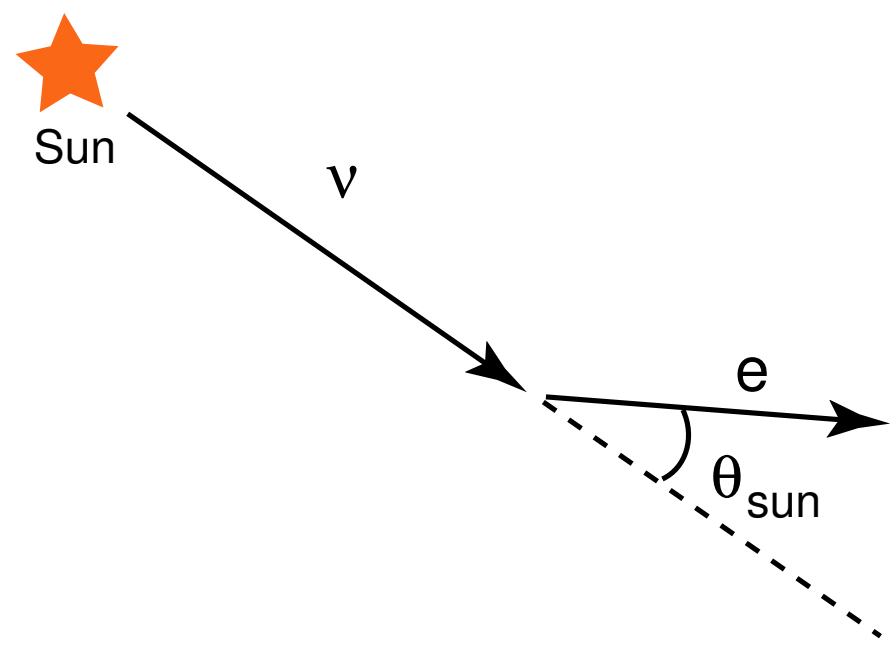

Figure 9.1: The definition of $\theta_{\text {sun }}$.

and $\mathrm{D}_{2} \mathrm{O}$, but the charged-current and neutral-current interactions only take place in $\mathrm{D}_{2} \mathrm{O}$. The direction of the electron in elastic-scattering reaction is highly correlated with the initial neutrino direction, while the neutral-current neutron is uncorrelated with the neutrino direction, and the charged-current electron is slightly anti-correlated. So, there are four variables that we can use to disentangle the neutrino signatures: the reconstructed radius $\left(R_{f i t}\right)$, the energy of the event $\left(n_{e f f}\right)$, the cosine of the angle of the event direction with respect to the Sun $\left(\cos \left(\theta_{\text {sun }}\right)\right.$, see Figure 9.1$)$, and the isotropy parameter $\left(\theta_{i j}\right)$. All of these distributions are depicted for the MC of three neutrino signals in Figure 9.2.

Background neutrons produced uniformly within the $\mathrm{D}_{2} \mathrm{O}$ will look identical to those from the NC interactions. Therefore, what we are really extracting here is the number of neutrons, from which we will have to subtract the number of internal background neutrons (which are tabulated in Chapter 8) to determine the number of NC events. Also, as was mentioned in Chapter 8, external neutrons (coming from the AV and $\mathrm{H}_{2} \mathrm{O}$ ) will be extracted 

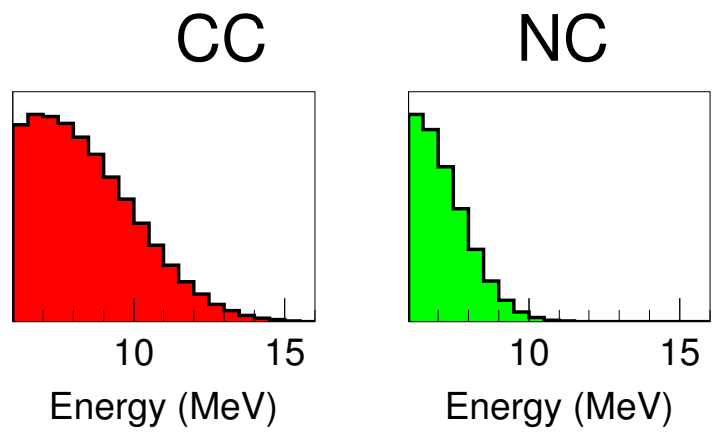

\section{ES}
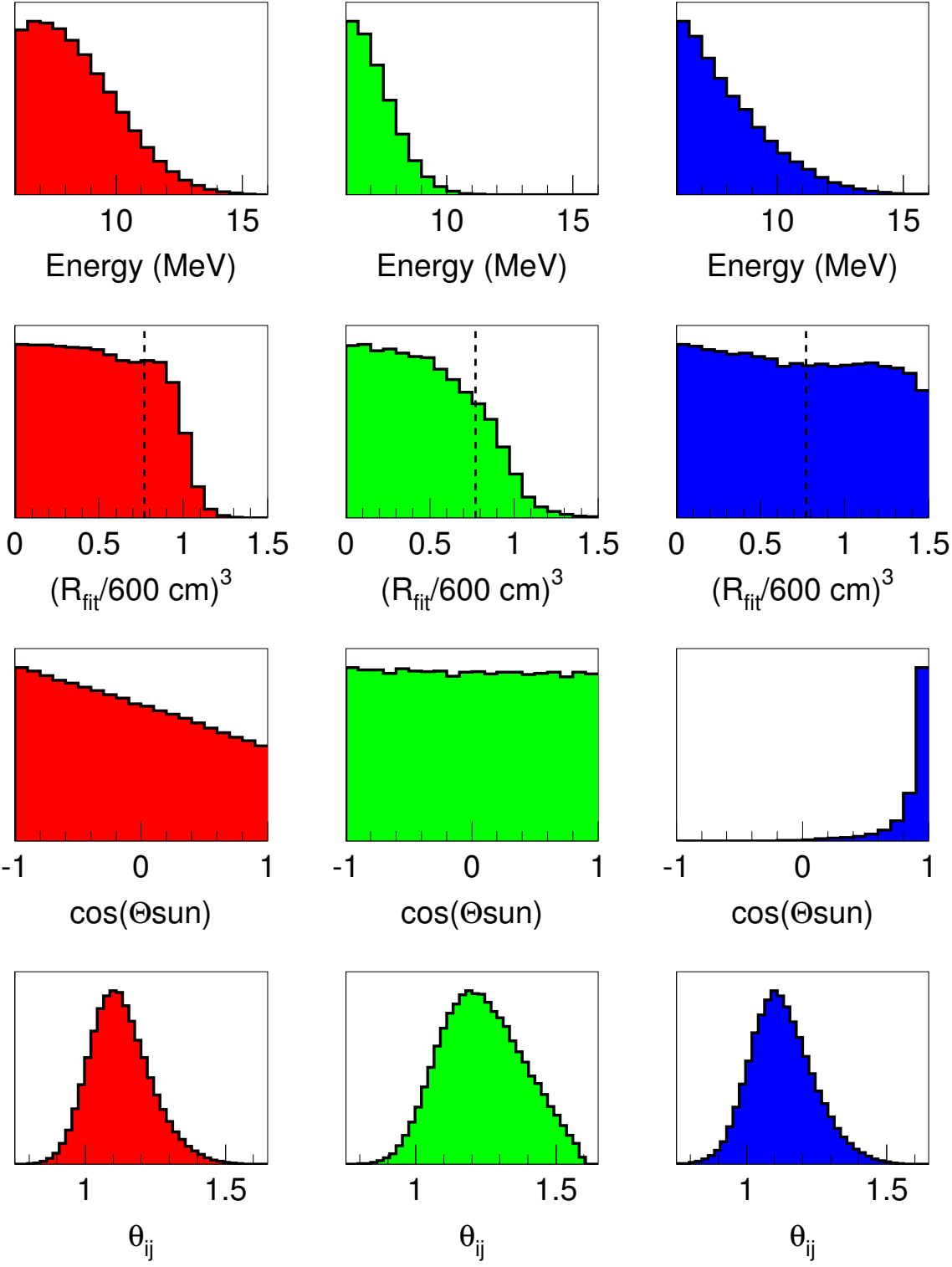

Figure 9.2: The expected energy, reconstructed radius, $\cos \left(\theta_{\text {sun }}\right)$, and $\theta_{i j}$ distributions for Monte-Carlo-simulated CC (left column), NC (middle column), and ES (right column) interactions. The dashed line on the $\mathrm{R}$ plots indicates the boundary of the fiducial volume. The y-axis scale is arbitrary. 
in the fit to the data along with the neutrino fluxes. These neutrons will look very similar to the neutrons from the neutral-current, but will have a different radial profile.

\subsection{Maximum Likelihood Algorithm}

To determine the number of neutrino events in the detector, a simultaneous maximum likelihood fit to the energy, $R_{f i t}, \cos \left(\theta_{s u n}\right)$, and $\theta_{i j}$ distributions has been performed. Here the fit determines 4 parameters: the number of $\mathrm{CC}, \mathrm{NC}, \mathrm{ES}$ and external neutron events, denoted as $N_{C C}, N_{N C}, N_{E S}$, and $N_{E N}$ respectively. We can begin with the general form for a binned extended maximum likelihood function (found in Chapter 5 of [120])

$$
\begin{array}{r}
\log \left(N_{t o t}, N_{C C}, N_{N C}, N_{E S}, N_{E N}\right)= \\
-N_{t o t}+\sum_{b=1}^{N B} D_{b} \log N_{b}\left(N_{C C}, N_{N C}, N_{E S}, N_{E N}\right)
\end{array}
$$

where we have the constraint on the total number of events $\left(N_{t o t}\right)$

$$
N_{t o t}=N_{C C}+N_{N C}+N_{E S}+N_{E N}
$$

Here $N B$ is the total number of bins, $D_{b}$ is the number of data events in bin $b$, and $N_{b}$ is the predicted number of events in bin $b$ (which depends on the values of $N_{C C}, N_{N C}, N_{E S}$, and $N_{E N}$ ). In our case, the predicted number of events for a given bin is determined from probability distribution functions derived from Monte Carlo simulations. The radial and $\cos \left(\theta_{\text {sun }}\right)$ pdfs are one dimensional. However, as was indicated in Chapter 5 , the shape of the $\theta_{i j}$ distribution varies with energy. Therefore, a joint two-dimensional pdf was used to describe the probability of obtaining an event with a given value of $\theta_{i j}$ and energy. So, the predicted number of events in bin $b$ (which is defined by the values $E_{b}, R_{b}, \cos \left(\theta_{\text {sun } b}\right.$ ), and 
$\left.\theta_{i j b}\right)$ can be expressed as

$$
\begin{aligned}
& N_{b}\left(N_{C C}, N_{N C}, N_{E S}, N_{E N}\right)= \\
& \quad N_{C C} R_{C C}\left(R_{b}\right) C_{C C}\left(\cos \left(\theta_{\text {sun } b}\right)\right) T_{C C}\left(E_{b}, \theta_{i j b}\right) \\
& +N_{N C} R_{N C}\left(R_{b}\right) C_{N C}\left(\cos \left(\theta_{\text {sun } b}\right)\right) T_{N C}\left(E_{b}, \theta_{i j b}\right) \\
& +N_{E S} R_{E S}\left(R_{b}\right) C_{E S}\left(\cos \left(\theta_{\text {sun } b}\right)\right) T_{E S}\left(E_{b}, \theta_{i j b}\right) \\
& +N_{E N} R_{E N}\left(R_{b}\right) C_{E N}\left(\cos \left(\theta_{\text {sun } b}\right)\right) T_{E N}\left(E_{b}, \theta_{i j b}\right)
\end{aligned}
$$

where $R_{x}, C_{x}$, and $T_{x}$ are the radial, $\cos \left(\theta_{\text {sun }}\right)$, and joint $\mathrm{E} / \theta_{i j}$ pdfs values for signal type $x$.

Equation 9.3 assumes that energy profile for the CC events is known (it factors into $\left.T_{C C}\right)$. However, if neutrinos are oscillating, this can cause distortion in the neutrino energy spectrum. So, it is desirable to not rely on CC energy information. To remove the dependence on the CC shape, the extraction will be performed in 10 energy bins, extracting a different value for the CC flux in each energy bin, for a total of 13 parameters to be determined in the fit. In this way the CC energy spectrum is unconstrained. For each CC energy bin, there will also be a distinct $\theta_{i j}$ pdf to account for the variation of $\theta_{i j}$ with the event energy. For the CC unconstrained spectrum fit, we can rewrite Equation 9.3 as

$$
\begin{aligned}
& N_{b}\left(N_{C C 1}, N_{C C 2}, \ldots, N_{C C 10}, N_{N C}, N_{E S}, N_{E N}\right)= \\
& \sum_{s=1}^{10} f_{s}\left(E_{b}\right) N_{C C s} R_{C C}\left(R_{b}\right) C_{C C}\left(\cos \left(\theta_{\text {sun } b}\right)\right) T_{C C s}\left(\theta_{i j} b\right) \\
& \quad+N_{N C} R_{N C}\left(R_{b}\right) C_{N C}\left(\cos \left(\theta_{\text {sun } b}\right)\right) T_{N C}\left(E_{b}, \theta_{i j b}\right) \\
& \quad+N_{E S} R_{E S}\left(R_{b}\right) C_{E S}\left(\cos \left(\theta_{\text {sun } b}\right)\right) T_{E S}\left(E_{b}, \theta_{i j} b\right) \\
& \quad+N_{E N} R_{E N}\left(R_{b}\right) C_{E N}\left(\cos \left(\theta_{\text {sun } b}\right)\right) T_{E N}\left(E_{b}, \theta_{i j b}\right)
\end{aligned}
$$


where

$$
f_{s}\left(E_{b}\right)= \begin{cases}1 & \text { if } E_{b} \text { is in CC energy bin } s \\ 0 & \text { otherwise }\end{cases}
$$

In principle the ES energy spectrum observed in the detector can also become distorted, but the electron energy spectrum for ES events is much less correlated with the incident neutrino energy. Kamiokande and Super-Kamiokande have not observed a significant distortion in the ES spectrum [36]. The number of ES events in the dataset is likely to be much smaller than the number of CC events (due to the smaller cross section). So the distortion of the ES event energy spectrum will not be considered here. The energy spectrum of the neutron captures is determined solely by nuclear physics, so the neutron energy response of the detector will not change if the incident neutrino energy spectrum is distorted.

The values for $N_{C C 1}, \ldots, N_{C C 10}, N_{N C}, N_{E S}, N_{E N}$ which maximize the likelihood given in Equation 9.1 (using Equation 9.4 for $N_{b}$ ), are the best fit values. The software used for the maximum likelihood extraction in this dissertation is an extension of a routine originally written by Scott Oser, used in [78]. The maximization (actually a minimization of the negative of Equation 9.1) is done using the CERNlib program MINUIT [121], using the SIMPLEX, MINIMIZE, and MIGRAD routines to perform the minimization, and using MINOS to determine the uncertainties on the parameters.

\subsection{The PDFs}

The probability density functions used in the maximum likelihood extraction for the CC, NC, ES, and external neutrons were generated from Monte Carlo simulations. The 
neutrino signals were generated at 100 times the predicted number based on the Standard Solar Model without oscillations. Here the Standard Solar Model (SSM) is that given by Bahcall, Pinsonneault, and Basu in [80], often referred to as the BP2000 model. The external neutrons were generated isotropically from the inner surface of the AV at a rate of 10,000 neutrons per day. The Monte Carlo simulations were generated on a run by run basis for the runs selected for the neutrino analysis, which ensures that the Monte Carlo simulations have the same temporal distribution as the data. The seasonal variations in the flux due to the variation of the Earth-Sun distance were also modeled. The Monte Carlo simulation was calibrated for energy using the relations given in Chapter 4 . The Level 1 instrumental backgrounds cuts (see Chapter 7) are not applied to the Monte Carlo simulations, but the Level 2 cuts are.

\subsection{Performance of Extraction Algorithm on Monte Carlo Simulated Datasets}

In order to test the performance of the signal extraction algorithm described in Section 9.2, 100 Monte Carlo simulated datasets were generated by randomly pulling events from the signal PDFs. The fake datasets each contain approximately $2324 \mathrm{CC}$ events $(\sim 35 \%$ SSM), 289 ES events ( 45\% SSM), 2118 NC events, and 130 external neutrons.

Table 9.1 summarizes the results of the extraction on the 100 test datasets. It lists the average difference between the fit and true number of events, the average fit uncertainty, and the average pull (defined as the fit difference divided by the fit uncertainty). In general the signal extraction algorithm performs well. The extracted values for the $\mathrm{CC}$ events and 
ES events are in good agreement with the input values. A slight bias is seen in the NC and external neutrons, with the signal extraction tending to slightly overestimate the number of external neutrons and underestimate the number of NC neutrons by approximately 25 events. This bias is believed to be caused by the fact that the signal extraction algorithm described does not take into account the variation of $\theta_{i j}$ with $\mathrm{r}$. This bias will be taken into account as a systematic uncertainty.

\begin{tabular}{|c|c|c|c|}
\hline Signal & Avg. Fit Error & Avg. Fit Uncert. & Avg. Pull \\
\hline \hline CC & -9.2 events & 86.4 events & -0.107 \\
NC & -23.3 events & 91.7 events & -0.254 \\
ES & 5.04 events & 27.7 events & 0.181 \\
Ext N & 27.5 events & 44.0 events & 0.62 \\
\hline
\end{tabular}

Table 9.1: The results of the signal extraction algorithm described in Section 9.2 for 100 $\mathrm{MC}$ test sets. Listed here is the average fit error (difference between the true and fit values), average fit uncertainty, and average pull (fit error/ fit uncertainty).

\subsection{The Dataset}

The salt dataset was taken between July 26, 2001 and August 28, 2003. For this neutrino analysis, 1212 data runs were selected, representing a total livetime of 391.4 days $[122]$.

The Level 1 and Level 2 data cleaning cuts have been applied to the events (See Chapter 7). Further cuts of $R_{f i t}<550 \mathrm{~cm}$ and $n_{e f f}>43.43$ hits (which is equivalent to a kinetic energy threshold of $5.5 \mathrm{MeV}$ ) have also been applied. A total of 4786 events remain in the dataset. 


\subsection{Extraction on Data}

The maximum likelihood signal extraction described in Section 9.2 has been applied to the dataset. The results of this fit are given in Table 9.2. In this fit, it is not possible to distinguish between internal neutron backgrounds (those that are uniformly distributed in the $\mathrm{D}_{2} \mathrm{O}$ ) and neutrons from the neutral-current neutrino interactions. The number of internal background neutrons was estimated in Chapter 8, and must be subtracted to obtain the number of $\mathrm{NC}$ events. The number of $\mathrm{CC}$ events is the sum of the values obtained for the 10 bins. Figures 9.3 to 9.6 show the $\cos \left(\theta_{\text {sun }}\right), \theta_{i j}, \mathrm{R}$, and $n_{\text {eff }}$ distributions of the data and the pdfs. The correlation coefficients are given in Table 9.3.

\begin{tabular}{|l|c|c|}
\hline Signal & Fit Value & Fit Uncert. \\
\hline \hline CC & 2232.8 events & 84.6 events \\
NC+Int. $\mathrm{n}$ & 2162.9 events & 90.7 events \\
( -Int. $\mathrm{n}$ & 137.7 events & 29.7 events) \\
NC & 2025.2 events & 90.7 events \\
ES & 261.4 events & 26.8 events \\
Ext $\mathrm{n}$ & 128.8 events & 43.6 events \\
\hline
\end{tabular}

Table 9.2: The results of the signal extraction algorithm described in Section 9.2 for the salt dataset. The number of internal background neutrons is given in Table 8.16 of Chapter 8 .

\begin{tabular}{|l||c|c|c|c|}
\hline Signal & CC & ES & NC & Ext. N \\
\hline \hline CC & 1.0 & -0.120 & -0.572 & -0.071 \\
ES & -0.120 & 1.0 & -0.068 & -0.16 \\
NC & -0.572 & -0.068 & 1.0 & -0.379 \\
Ext. N & -0.071 & -0.016 & -0.379 & 1.0 \\
\hline
\end{tabular}

Table 9.3: The correlation coefficients for the maximum likelihood fit to the salt dataset. 


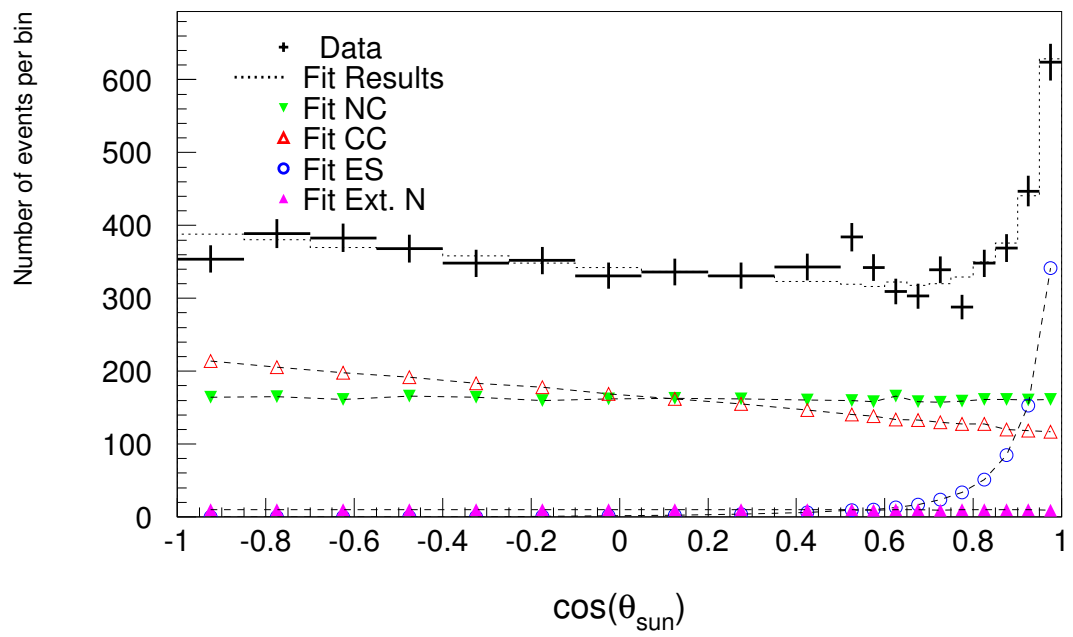

Figure 9.3: The $\cos \left(\theta_{\text {sun }}\right)$ distribution for the data and the results of the maximum likelihood fit. Here an equal number of bins have been used above and below $\cos \left(\theta_{\text {sun }}\right)=0.5$.

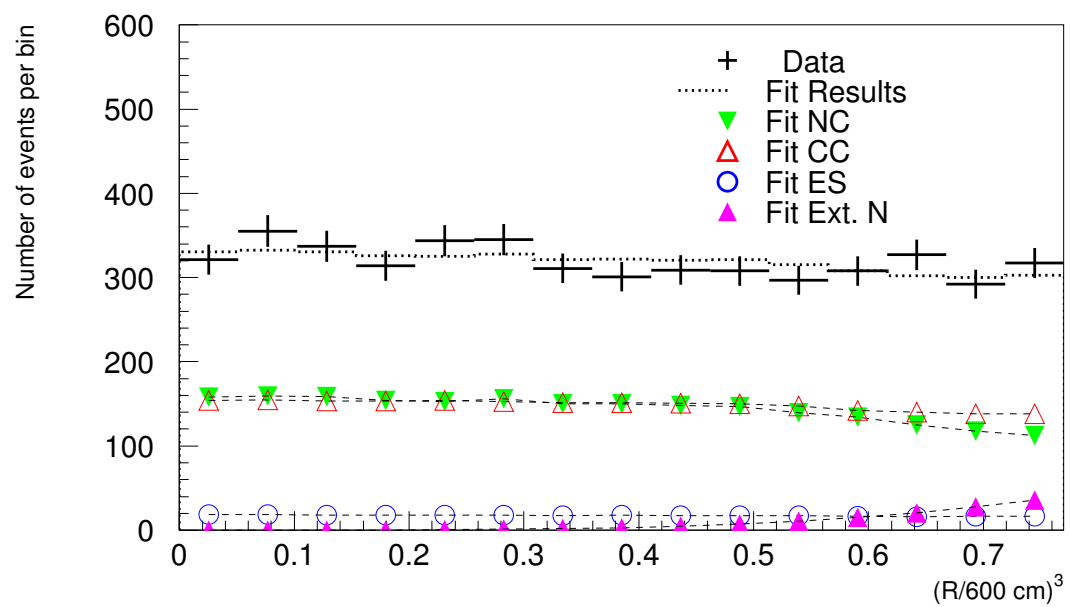

Figure 9.4: The $\mathrm{R}_{\text {fit }}^{3}$ distribution for the data and the results of the maximum likelihood fit. 


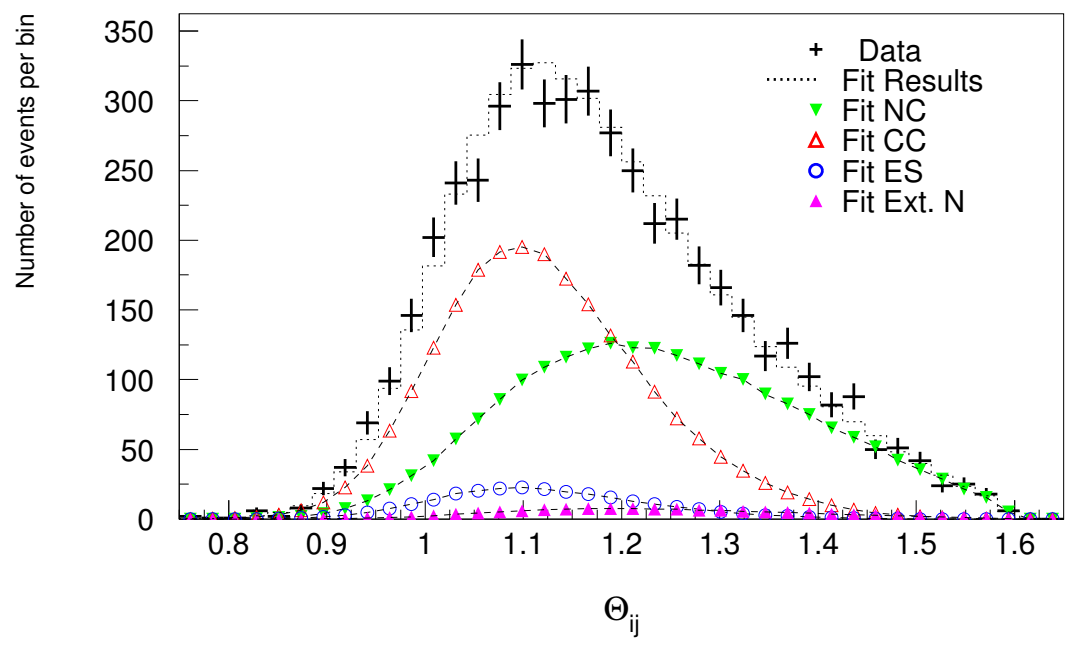

Figure 9.5: The $\theta_{i j}$ distribution for the data and the results of the maximum likelihood fit.

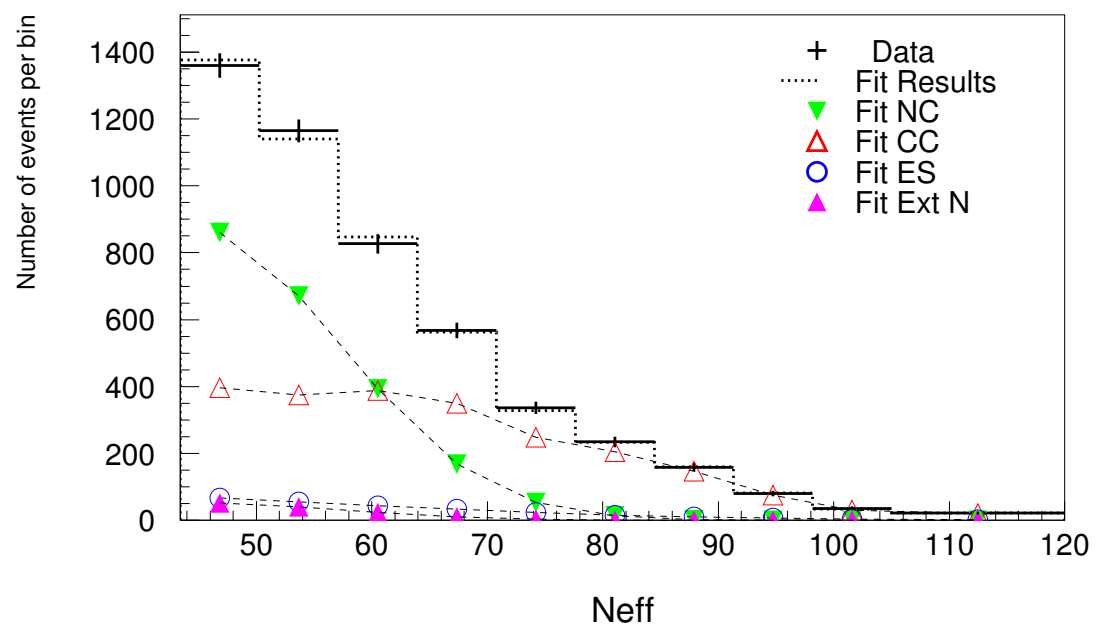

Figure 9.6: The $n_{\text {eff }}$ distribution for the data and the results of the maximum likelihood fit. The $n_{\text {eff }}$ shapes for the external neutrons, ES, and NC signals were constrained in the fit, while the CC shape was free to vary. 


\subsection{Systematics}

Table 9.4 contains all of the systematic uncertainties on the extracted number of events given in Table 9.2. In general the systematic uncertainties were evaluated by slightly modifying the PDFs input into the fit, and then refitting the data. The difference in the resulting fit value is taken to be the systematic uncertainty on the fit results. For the selected fiducial volume and energy threshold, the systematic uncertainties were evaluated by slightly varying these choices. The uncertainty on the mean of the isotropy distribution was evaluated by shifting the PDFs up and down relative to the data. The uncertainty on the width of a distribution (such as the angular resolution, the energy resolution, or the width of the isotropy distributions) was evaluated by randomly smearing out the MC PDFs by a Gaussian and then refitting the data. For example, the MC electron energy response was smeared by a Gaussian with a width of 2.1 hits to reflect the difference seen between the ${ }^{16} \mathrm{~N}$ data and $\mathrm{MC}$ in Chapter 4 . It is only possible to smear the MC to make it wider, not narrower. So, the resulting shift in the extracted number of events was assumed to be symmetric. The uncertainty obtained in Chapter 6 for the neutron detection efficiency only affects the NC signal. Many of the Čerenkov backgrounds estimated in Chapter 8 are a systematic uncertainty for both the electrons and neutrons because the $\beta \gamma$ decays can have multiple particles, making them more isotropic than a single $\beta$ event. Since the ES neutrino signal is well constrained in the fitting process by the $\cos \left(\theta_{\text {sun }}\right)$ distribution and the backgrounds are distributed uniformly in direction, background events are unlikely to affect the extracted ES flux. So, the background events were not included as systematic uncertainties on the ES flux. The theoretical cross section uncertainties were evaluated 
in [123] and the uncertainty is dominated by uncertainties in the effective field theory calculations.

Table 9.4: This table summarizes the systematic uncertainties on the maximum likelihood fit to the data. It gives the percentage changes on the various neutrino signals. The statistical uncertainties and the theoretical uncertainties on the cross sections are given at the end of the table.

\begin{tabular}{|c|c|c|c|c|c|}
\hline \multirow[b]{2}{*}{ Source } & \multirow{2}{*}{$\begin{array}{l}\text { Uncert. } \\
\text { Value }\end{array}$} & \multicolumn{3}{|c|}{$\%$} & \multirow{2}{*}{$\begin{array}{l}\text { Chapt. } \\
\text { Ref. }\end{array}$} \\
\hline & & $\Delta \mathrm{CC}$ & $\Delta \mathrm{NC}$ & $\Delta \mathrm{ES}$ & \\
\hline Fid. Volume & $\pm 2 \mathrm{~cm}$ & $\pm_{1.2}^{1.1}$ & $\pm_{1.4}^{0.9}$ & $\pm_{2.1}^{0.8}$ & 3.5 \\
\hline Angular Res. & & \pm 0.4 & \pm 0.1 & \pm 4.7 & 3.5 \\
\hline Recon. Effic. & $\pm 0.23 \%$ & $\pm 0.23 \%$ & $\pm 0.23 \%$ & $\pm 0.23 \%$ & 3.5 \\
\hline E Thresh. & $\pm 0.81 \%$ & $\pm_{1.3}^{0.6}$ & $\pm_{1.9}^{2.3}$ & $\pm_{0.2}^{1.4}$ & 4.6 \\
\hline E res. $\mathrm{e}^{-}$ & & \pm 0.09 & \pm 0.4 & \pm 0.6 & 4.6 \\
\hline E res. $n$ & & \pm 0.4 & \pm 0.4 & \pm 0.04 & 4.6 \\
\hline$\theta_{i j}$ mean $\mathrm{e}^{-}$ & \pm 0.006 & $\pm_{3.1}^{2.9}$ & $\pm_{3.1}^{3.3}$ & $\pm_{1.3}^{1.0}$ & 5.8 \\
\hline$\theta_{i j}$ width $\mathrm{e}^{-}$ & & \pm 0.6 & \pm 0.7 & \pm 0.7 & 5.8 \\
\hline$\theta_{i j}$ mean $\mathrm{n}$ & \pm 0.002 & $\pm_{1.7}^{1.5}$ & $\pm_{1.6}^{1.8}$ & \pm 0.5 & 5.8 \\
\hline$\theta_{i j}$ width $\mathrm{n}$ & & \pm 0.5 & \pm 0.4 & \pm 0.3 & 5.8 \\
\hline N Efficiency & $\pm_{5.1}^{4.1 \%}$ & - & $\pm_{5.1}^{4.1 \%}$ & - & 6.6 \\
\hline Inst. Back & 3 Evs & \pm 0.1 & \pm 0.1 & \pm 1.1 & 7.3 \\
\hline Lev 1 Sacrifice & & $\pm_{0.15}^{0.09 \%}$ & $\pm_{0.15}^{0.09}$ & $\pm_{0.15}^{0.09 \%}$ & 7.2 .1 \\
\hline Lev 2 Sacrifice & & \pm 0.006 & \pm 0.016 & \pm 0.018 & 7.2 .2 \\
\hline Int. Cer Bkg & 3.48 Evs & -0.2 & -0.2 & - & 8.8 \\
\hline Int. n Bkg Uncert. & 29.7 Evs & - & \pm 1.5 & - & 8.8 \\
\hline PMT Cer Bkg & $<17.4$ evs & -0.8 & -0.9 & - & 8.8 \\
\hline Ext. $\gamma$-rays & $<8.42$ evs & -0.4 & -0.4 & - & 8.8 \\
\hline$\mu$ Cer Bkgs & $<2.1$ Evs & -0.1 & -0.1 & - & 8.8 \\
\hline AV Events & $<12.3$ Evs & -0.6 & -0.6 & - & 8.8 \\
\hline Other Bkg Events & 8.5 Evs & -0.4 & -0.4 & - & 8.8 \\
\hline Fit $\mathrm{n} / \mathrm{NC}$ bias & 27.5 Evs & - & +1.35 & - & 9.4 \\
\hline Total Exp. Uncert. & & $\pm_{4.0}^{3.8}$ & $\pm \pm_{7.4}^{6.2}$ & \pm 5.4 & \\
\hline Stat. Uncert. & & \pm 3.8 & \pm 4.5 & \pm 10.3 & \\
\hline Cross Sec. Uncert. & & \pm 1.2 & \pm 1.1 & \pm 0.5 & \\
\hline
\end{tabular}

In this chapter, the number of neutrino events from the various solar neutrino interactions were evaluated along with the systematic uncertainties on these numbers. In the next chapter we will convert these numbers into fluxes and interpret what consequences 
these measurement have for neutrino physics. 


\section{Chapter 10}

\section{Interpretation of Results and}

\section{Conclusions}

\subsection{Neutrino Fluxes}

In the previous chapter we obtained the numbers of neutrino events in the SNO dataset within $550 \mathrm{~cm}$ of the center of the detector and above $5.5 \mathrm{MeV}$ of visible energy. To convert this into a flux, we will use the number of events predicted by the Monte Carlo simulations. This requires a number of small corrections, which are summarized in Table 10.1.

Level 1 Cut Livetime: The Level 1 cuts in Chapter 7 (in particular the muon follower cut) slightly reduce the detector livetime to $98.2 \%$ of the full run time [122]. The $\mathrm{MC}$ was generated based on the full run time, so the predicted number of $\mathrm{MC}$ events must be reduced by $98.2 \%$. 
Level 1 Cut Events: The Level 1 data cleaning cuts were applied to the data, but not to the MC. These cuts keep $99.63 \%$ of the electron events and $99.60 \%$ of the neutron events in the data, so the MC must be scaled down to reflect this.

MC Geometry Errors: When the MC events are generated, a small fraction of the events terminate due to fatal geometry errors. So, it is necessary to scale up the MC prediction.

CC on ${ }^{17,18} \mathbf{O}, \mathbf{N a}, \mathbf{C l}$ : Solar neutrinos can also undergo CC interaction on $\mathrm{Na}$, $\mathrm{Cl}$, and the trace amounts of ${ }^{17} \mathrm{O}$ and ${ }^{18} \mathrm{O}$ in the water. These will look very similar to the $\mathrm{CC}$ interaction on $\mathrm{D}$, but they are not included in the MC simulations. So, the predicted number of CC events must be scaled up slightly [111].

Density of $\mathbf{D}_{2} \mathbf{O}$ : The density of the $\mathrm{D}_{2} \mathrm{O}$ used in the $\mathrm{MC}$ is not at the correct temperature. Also SNOMAN incorrectly uses the figure of $6.023 \times 10^{31}$ deuterons to be the total number in the spherical part of the vessel and the neck, when this figure really reflects the number in the spherical part of the vessel alone. So, the number density of deuterons must be scaled up [124]. For elastic-scattering, the number density of electrons is important. SNOMAN assumes that there are exactly 10 electrons per mass of a heavy water molecule. In reality, there are trace amounts of ${ }^{1} \mathrm{H},{ }^{17} \mathrm{O}$ and ${ }^{18} \mathrm{O}$, so the correction for the ES interactions is slightly different than that for the CC and NC interactions [124].

$\mathbf{g}_{A}$ : To evaluate the neutrino-deuteron cross sections, SNO uses the effective field theory calculations of Butler, Chen and Kong [125]. However, it was pointed out by Beacom and Parke [126] that these calculations were not using the most up to date value of $\mathrm{g}_{A}$, the electroweak axial coupling constant, so a correction has been applied to the MC [123]. 
$\mathbf{L}_{1, A}:$ In the next-to-next-to leading order cross section calculations [125], the axial two-body exchange is described by a parameter $\mathrm{L}_{1, A}$. The most up to date value for this term leads to a slight correction to the cross section [127].

Radiative Corrections: The latest radiative correction calculations [128] for the neutrino-deuteron interactions are not yet implemented in SNOMAN, leading to another small correction [123]. Radiative corrections for the elastic-scattering interaction have been evaluated by Yasuo Takeuchi [129].

\begin{tabular}{|c|c|c|c|}
\hline Source & $\mathrm{CC}$ & $\mathrm{ES}$ & $\mathrm{NC}$ \\
\hline Level 1 Cut Livetime & 0.982 & 0.982 & 0.982 \\
Level 1 Cut Events & 0.9963 & 0.9963 & 0.9960 \\
MC Geometry Errors & 1.0047 & 1.0028 & 1.0034 \\
$\mathrm{CC}$ on ${ }^{17,18} \mathrm{O}, \mathrm{Na}, \mathrm{Cl}$ & 1.0081 & - & - \\
Density of $\mathrm{D}_{2} \mathrm{O}$ & 1.012 & 1.013 & 1.012 \\
$g_{A}$ & 1.0111 & - & 1.0111 \\
$L_{1 A}$ & 0.984 & - & 0.979 \\
Radiative Corrections & 1.026 & 0.971 & 1.0154 \\
\hline Total & 1.023 & 0.965 & 0.998 \\
\hline \hline
\end{tabular}

Table 10.1: Corrections to MC Predicted Event Rates

With the MC correction factors calculated, we can now convert the observed number of events into a flux. The MC generated here used the Bahcall-Pinnsoneault 2000 solar model (BP2000), which predicts a total neutrino flux of $5.05 \times 10^{6} \mathrm{~cm}^{-2} \mathrm{~s}^{-1}$ [80]. Table 10.2 gives the predicted numbers of events that correspond to this flux for each neutrino signal. Note that this flux does not assume any distortions to the neutrino energy spectrum. So, the fluxes quoted in this dissertation represent the total neutrino flux assuming no distortions to the energy spectrum.

Table 10.3 gives the numbers of observed events in the dataset. By scaling to the 


\begin{tabular}{|c|c|c|c|c|}
\hline Type & MC Flux & \# Events & Correc. & Correc. \# Events \\
\hline CC & $5.05 \times 10^{6} \mathrm{~cm}^{-2} \mathrm{~s}^{-1}$ & 6449.5 & 1.023 & 6597.8 \\
ES & $5.05 \times 10^{6} \mathrm{~cm}^{-2} \mathrm{~s}^{-1}$ & 618.8 & 0.965 & 597.1 \\
NC & $5.05 \times 10^{6} \mathrm{~cm}^{-2} \mathrm{~s}^{-1}$ & 2027.7 & 0.998 & 2023.6 \\
\hline
\end{tabular}

Table 10.2: The conversion between flux and the expected number of events in the 391-day dataset (with $R_{\text {fit }}<550 \mathrm{~cm}$, and $\mathrm{E}_{v i s}>5.5 \mathrm{MeV}$ ) assuming no neutrino oscillation. The correction factors come from Table 10.1.

values in Table 10.2, they can be converted into the corresponding flux values, also included in Table 10.3 .

\begin{tabular}{|c|c|c|}
\hline Type & \# Events & Flux \\
\hline CC & 2232.8 & $\left[1.71 \pm 0.065\right.$ (stat.) $\pm_{0.068}^{0.065}$ (sys.) \pm 0.02 (theor. $\left.)\right] \times 10^{6} \mathrm{~cm}^{-2} \mathrm{~s}^{-1}$ \\
ES & 261.4 & $\left[2.21 \pm 0.22\right.$ (stat.) $\pm_{0.12}^{0.11}$ (sys.) \pm 0.01 (theor. $\left.)\right] \times 10^{6} \mathrm{~cm}^{-2} \mathrm{~s}^{-1}$ \\
NC & 2025.2 & {$\left[5.05 \pm 0.23\right.$ (stat.) $\pm_{0.37}^{0.31}$ (sys.) \pm 0.06 (theor.) $] \times 10^{6} \mathrm{~cm}^{-2} \mathrm{~s}^{-1}$} \\
\hline
\end{tabular}

Table 10.3: The extracted number of neutrino events in the dataset and the corresponding flux value.

\subsection{Interpretation of Fluxes}

The neutrino fluxes in Table 10.3 provide strong evidence for neutrino transformations. The flux of electron neutrinos observed via the CC interaction is over $7 \sigma$ smaller than the flux of all active neutrino flavors observed via the NC interaction, and is $2 \sigma$ smaller than the mostly electron neutrino flux observed via the ES interaction. This strongly implies that the total neutrino flux from the Sun at the Earth is not equal to the electron flux. It appears that neutrinos are changing flavors between the core of the Sun and the Earth. This is strong evidence that at least some neutrino flavors must have a non-zero mass. Figure 10.2 shows the total neutrino flux plotted against the electron neutrino flux for the CC and NC fluxes obtained in this dissertation. The Super-Kamiokande measurement 
of the solar neutrino flux via the ES interaction has been included as well. The $1 \sigma$ band for the Super-Kamiokande measurement just touches the $1 \sigma$ ellipse for the combined SNO CC and NC measurement.

The observed flux of active neutrinos is also in very good agreement with the BP2000 Standard Solar Model ${ }^{8} \mathrm{~B}$ predicted flux of $5.05 \pm_{0.81}^{1.01} \times 10^{6} \mathrm{~cm}^{-2} \mathrm{~s}^{-1}$ [80]. It should also be noted here that the most recent Standard Solar Models (BP04 and BP04+) predict

${ }^{8} \mathrm{~B}$ fluxes of $5.79 \pm 1.33 \times 10^{6} \mathrm{~cm}^{-2} \mathrm{~s}^{-1}$ and $5.26 \pm 1.21 \times 10^{6} \mathrm{~cm}^{-2} \mathrm{~s}^{-1}$ respectively [130]. The changes made include new measurements of nuclear interaction cross sections, a refined equation of state, and new inputs for the surface chemical composition of the Sun (included in $\mathrm{BP} 04+$, and not in BP04). The measurement of the total neutrino flux presented in this dissertation is still within the theoretical uncertainty on the new predicted values.

\subsection{Neutrino Oscillation Parameters}

In Chapter 1, the basic equations governing the probability of neutrino oscillation were given. Two free fundamental parameters - a mixing angle $(\theta)$ and the difference in the square of the neutrino masses $\left(\Delta m^{2}\right)$ - describe two-flavor oscillations in vacuum. When neutrinos travel through matter an additional phase rotation, which depends on the electron density of the material, is introduced into the oscillation probability equations. (See

Section 1.6.) We can now examine the possible allowed regions of the $\theta-\Delta m^{2}$ parameter space which can account for the flux results given in this chapter.

The oscillation contours in this dissertation were generated using the QPhysics software package, which was also used to generate the contour plots in previous SNO pub- 


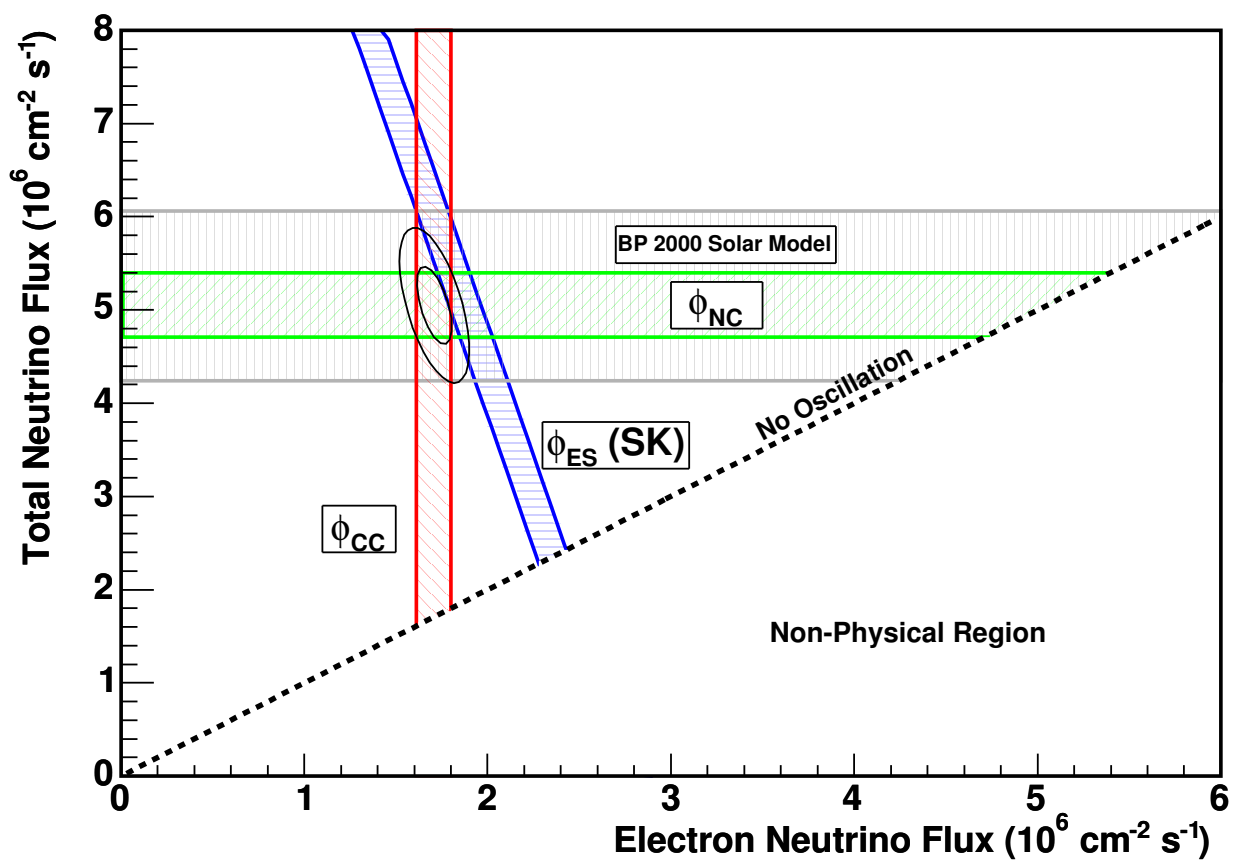

Figure 10.1: The figure shows $1 \sigma$ regions for the total neutrino flux vs the electron neutrino flux for the CC measurement from this dissertation (vertical band), the NC measurement from this dissertation (horizontal band), and the Super-Kamiokande measurement of the ES flux from [36](diagonal band). Also shown is the region predicted from the BP 2000 solar model [80]. The ellipses shown here are the $1 \sigma$ and $2 \sigma$ regions for the combined SNO $\mathrm{CC}$ and $\mathrm{NC}$ measurement, taking into account the anti-correlation between these two flux values. For the case of no oscillations, the total neutrino flux equals the electron neutrino flux. It is not possible for the electron neutrino flux to be less than the total neutrino flux. 
lications [131] [132]. Figure 10.3 shows a flow chart outlining the principal elements of the calculation. For the plots in this dissertation, a grid of $101 \log$ bins in $\tan ^{2} \theta$ (from $10^{-4}$ to 10) and $181 \log$ bins in $\Delta m^{2}$ (from $10^{-3} \mathrm{eV}^{2}$ to $10^{-12} \mathrm{eV}^{2}$ ) was used. The first step in the calculation is to calculate the survival probability in the Sun for an electron neutrino for each grid point. This is a complicated calculation involving matter (MSW) effects. After the neutrinos exit the Sun, they essentially undergo only vacuum oscillations before they reach the Earth. So, the next step of the calculation combines the solar MSW probabilities with the vacuum probabilities for each grid point. When the neutrinos reach the Earth, they can undergo matter oscillations in the Earth again. So, the survival probability in the Earth is then estimated for each grid point. This oscillation depends on the neutrino path length in the Earth, which depends on the time of day. So, the solar zenith angle distribution of the actual SNO dataset is input into the calculation as well. It is then possible to estimate the expected event rates in the SNO detector. For each grid point, a $\chi^{2}$ calculation is performed for the expected rate and the observed rate in the SNO data (taking into account the systematic and statistical uncertainties). This grid of $\chi^{2}$ values can then be used to make a plot of the allowed regions.

Figure 10.3 shows the allowed regions in $\tan ^{2} \theta-\Delta m^{2}$ space using only the SNO salt flux results given in this dissertation. Since the predicted solar neutrino flux is rather uncertain $(\sim \pm 20 \%)$, the ${ }^{8} \mathrm{~B}$ solar neutrino flux was not constrained in the fit, but was allowed to vary at each grid point to whatever value gave the best $\chi^{2}$ value for that grid point.

Figure 10.3 shows a similar plot, but with the previously published SNO pure $\mathrm{D}_{2} \mathrm{O}$ 
MODEL

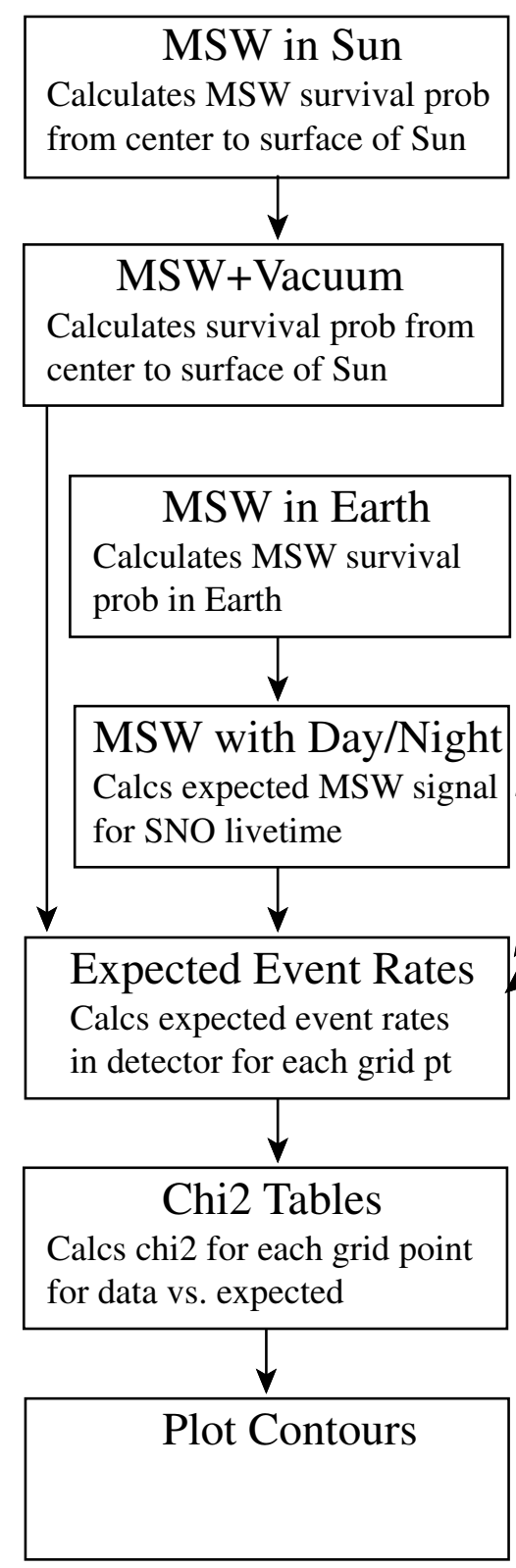

DATA

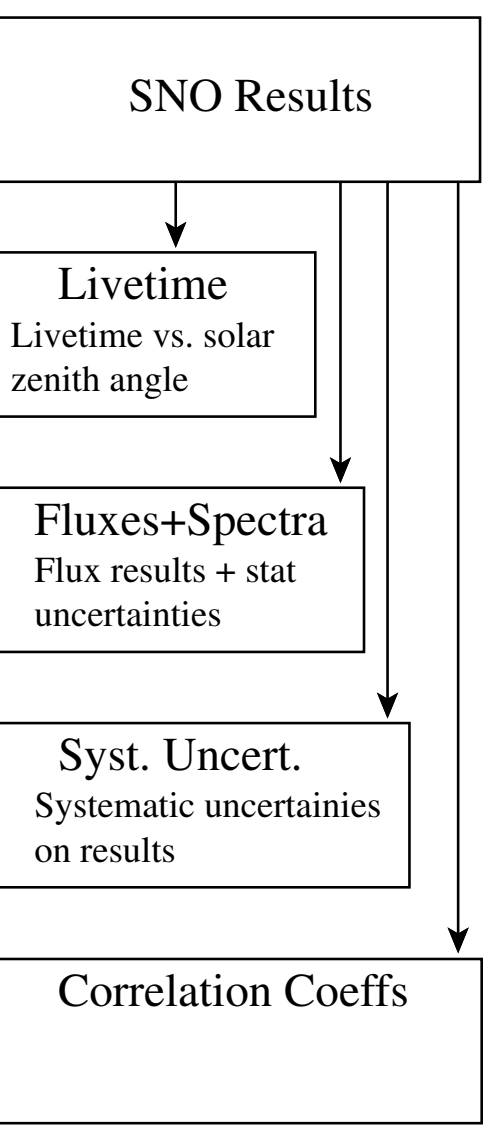

Figure 10.2: This schematic outlines the process used to determine the allowed regions for the neutrino oscillation parameters. 
fluxes [79] and day and night energy spectra [131] included in the fit along with the salt flux results from this dissertation. The combined SNO results only allow for a few regions of parameter space. The best fit point, indicated on Figure 10.3 by a star, corresponds to $\tan ^{2} \theta$ of $4.47 \times 10^{-1}$ and $\Delta m^{2}$ of $5.01 \times 10^{-5} \mathrm{eV}^{2}$. At that point the best fit value for the ${ }^{8} \mathrm{~B}$ flux is 1.017 times the BP2000 Standard Solar Model value.

Compare the allowed regions in Figure 10.3 to those in Figure 1.5, which shows the allowed regions in mixing parameter space obtained from all solar neutrino experiments, circa 2001, prior to the publication of any SNO results. Several discrete islands in the $\tan ^{2} \theta-\Delta m^{2}$ space were allowed and these are referred to as the large mixing-angle region (LMA), the small mixing-angle region (SMA), the low $\Delta m^{2}$ region (LOW), the vacuum oscillation region (VAC), and the "just-so" region. In the SNO-only plot in Figure 10.3, the LMA and LOW solutions survive, but the "just-so", SMA and VAC regions are almost entirely eliminated. The best-fit point for the SNO measurements is in the LMA region.

It is also worth pointing out that the $99.73 \%$ confidence levels do not intersect the line $\tan ^{2} \theta=1$. If solar neutrino mixing were maximal (i.e. the mixing angle is $45^{\circ}$ ), then the best fit regions would lie around $\tan ^{2} \theta=1$. It now appears that the value of $\theta$ is large, but not maximal.

\subsection{Conclusions}

This dissertation presented a determination of the solar neutrino flux inferred via the charged-current, neutral-current, and elastic-scattering interactions with salted heavy water in the Sudbury Neutrino Observatory. The flux seen via the neutral-current inter- 


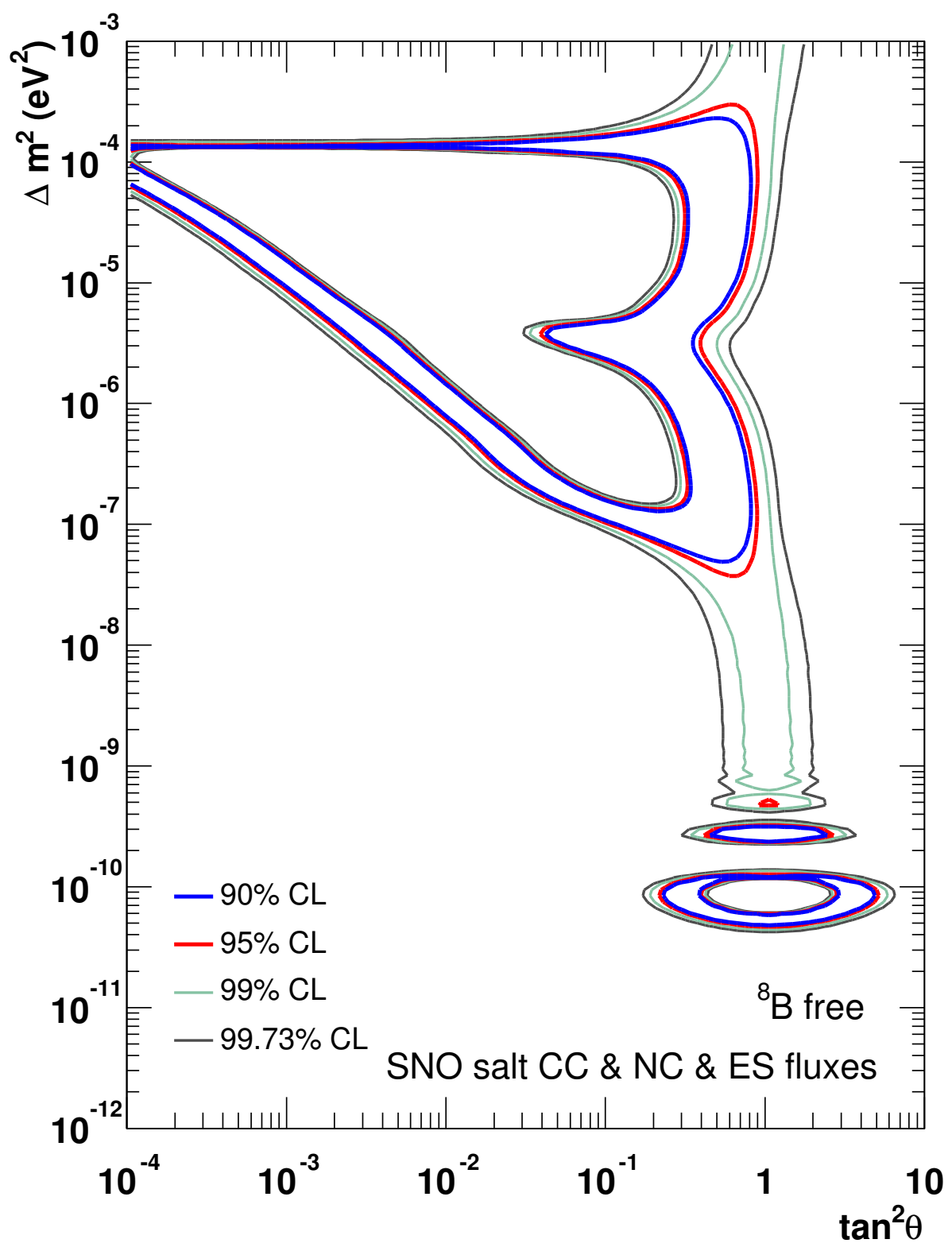

Figure 10.3: The areas enclosed by the contour lines represent the allowed $\tan ^{2} \theta$ and $\Delta m^{2}$ values for the SNO salt flux results given in this dissertation. 


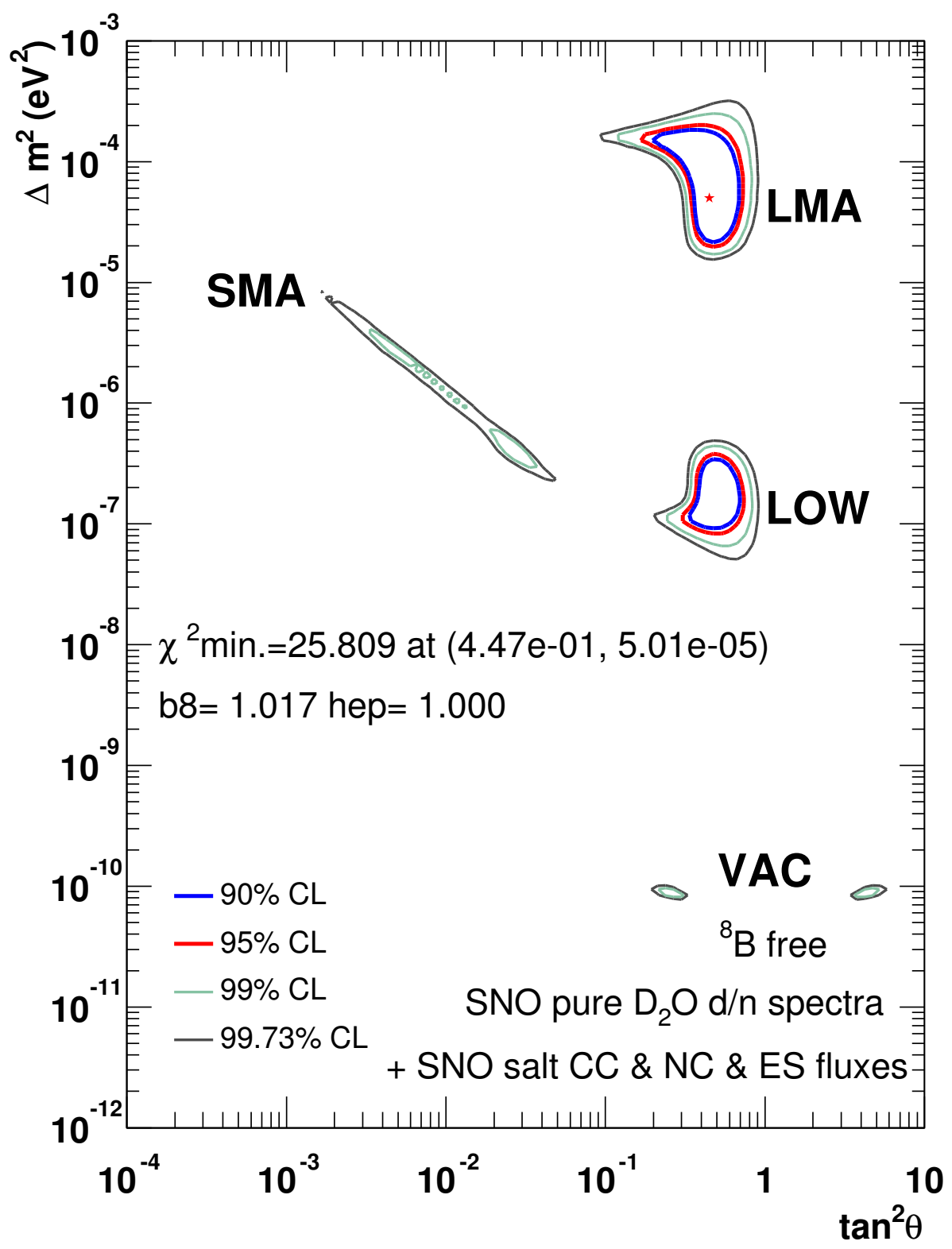

Figure 10.4: The areas enclosed by the contour lines represent the allowed $\tan ^{2} \theta$ and $\Delta m^{2}$ values for the combined SNO salt flux results and the previously published results from the pure $\mathrm{D}_{2} \mathrm{O}$ phase in [79] and [131]. The best fit point is indicated with a star. 
action (which is sensitive to all neutrino flavors) is over $7 \sigma$ larger than the flux seen via the charged-current interaction (which is sensitive only to electron neutrinos). The total flux observed via the neutral-current interaction is also in very good agreement with the predicted solar neutrino flux in the Standard Solar Model of the Sun.

The long-standing mystery of the missing solar neutrinos has finally been resolved. Neutrinos appear to be undergoing flavor transformations; only electron neutrinos are produced in the Sun and other neutrino flavors reach the Earth. If one takes into account all flavors, then the observed neutrino flux is in good agreement with the predicted flux.

A two-neutrino oscillation flavor analysis has been performed using the SNO flux results. This work indicates the neutrino oscillation parameters that can account for the observed neutrino fluxes are likely to reside in either the so-called Large Mixing Angle (LMA) region or in the LOW region. The vacuum oscillation and SMA solutions are not allowed at the $95 \%$ confidence level. The results favor a large $\left(\sim 35^{\circ}\right)$, but non-maximal, mixing angle. This mixing angle, often called $\theta_{12}$, is just one of three mixing angles which are required for three neutrino flavors. The angle that dominates atmospheric neutrino oscillation, $\theta_{23}$, has a best fit value of $45^{\circ}$, and a lower limit of $\sim 35^{\circ}$ at the $99 \%$ confidence level according to the latest Super-Kamiokande results [133]. The third mixing angle, $\theta_{13}$, has not yet been measured, but a $90 \% \mathrm{CL}$ upper limit of $\sim 9^{\circ}$ has been found by the $\mathrm{CHOOZ}$ reactor neutrino experiment [134]. Two of the three neutrino mixing angles are quite large. In this respect, neutrino flavor mixing is very different from quark flavor mixing, where the largest angle is approximately $13^{\circ}$ and the other two angles are both less than $2.5^{\circ}[18]$.

Further hints that the LMA region is the solution come from the KamLAND 
reactor neutrino experiment. Only the LMA region of the oscillation parameter space can account for the disappearance of reactor anti-neutrinos and the distortion of the antineutrino energy spectrum seen in the KamLAND detector [44]. Through solar and reactor neutrino experiments, a great deal of progress has been made in the past five years to restrict the parameters required to explain solar neutrino oscillations.

The fact that neutrinos change flavors is also a piece of very strong evidence that at least some of the neutrino flavors must have a non-zero mass. This is of interest because even a tiny neutrino mass can contribute significantly to the mass density of the universe due to the great abundance of neutrinos. The results in this dissertation favor a $\Delta m^{2}$ in the range of $10^{-5}$ to $10^{-4} \mathrm{eV}^{2}$ to explain the oscillation of the electron neutrinos from the Sun. Strong evidence for atmospheric muon neutrino oscillation has been reported by the Super-Kamiokande collaboration $[43,133]$. The most recent Super-Kamiokande atmospheric neutrino results require a $\Delta m^{2}$ value that is larger than $1.9 \times 10^{-3} \mathrm{eV}^{2}$ [133]. This means that at least one neutrino flavor must have a mass on the order of $0.04 \mathrm{eV}$. The density of relic neutrinos from the Big Bang is approximately 100 per $\mathrm{cm}^{3}$ per flavor [22]. Assuming that neutrinos and anti-neutrinos have the same mass, the mass density of neutrinos and anti-neutrinos in the universe must be at least $8 \mathrm{eV}$ per $\mathrm{cm}^{3}$. The baryon density of the universe is at most $242 \mathrm{eV}$ per $\mathrm{cm}^{3}$ [18] and the total matter density of the universe is at most $3200 \mathrm{eV}$ per $\mathrm{cm}^{3}$ [18]. Therefore, the neutrino mass density of the universe must be at least $3 \%$ of the baryon mass density and at least $0.25 \%$ of the matter density of the universe.

Despite the resolution of the solar neutrino problem, many unanswered questions 
remain about neutrinos, their masses, and their properties. What are the absolute neutrino masses and why are they so small? Do neutrino masses affect cosmology? What is the order of the mass hierarchy? Why are the mixing angles so large? Why are neutrinos only left-handed? Are neutrinos their own anti-particle?

We have only begun to scratch the surface in our understanding of neutrino masses and mixings. Hopefully many exciting discoveries await us in the field of neutrino physics over the next few decades. 


\section{Appendix A}

\section{KamLAND Electronics}

\section{A.1 Waveform Digitization in the KamLAND Detector}

The Kamioka Liquid scintillator Anti-Neutrino Detector (KamLAND), currently in operation in Japan, is a long baseline neutrino oscillation experiment that uses Japanese commercial nuclear power reactors as the neutrino source. The neutrino target consists of 1 kton of liquid scintillator, encased in a $13 \mathrm{~m}$ diameter spherical balloon. This balloon sits inside an 18-m spherical stainless steel containment vessel, filled with dodecane and isoparaffin oils to provide shielding from external radiation. The containment vessel also supports approximately 1900 photo-multiplier tubes, which look into the liquid scintillator target. A side view of the detector is shown in Figure A.1. More details on the detector design can be found in [135].

The primary goal of the experiment is to measure the flux of reactor anti-neutrinos coming from nuclear power plants in the vicinity of the detector. There are 51 nuclear reactors in Japan within $850 \mathrm{~km}$ of the KamLAND site, and $80 \%$ of the anti-neutrino flux 


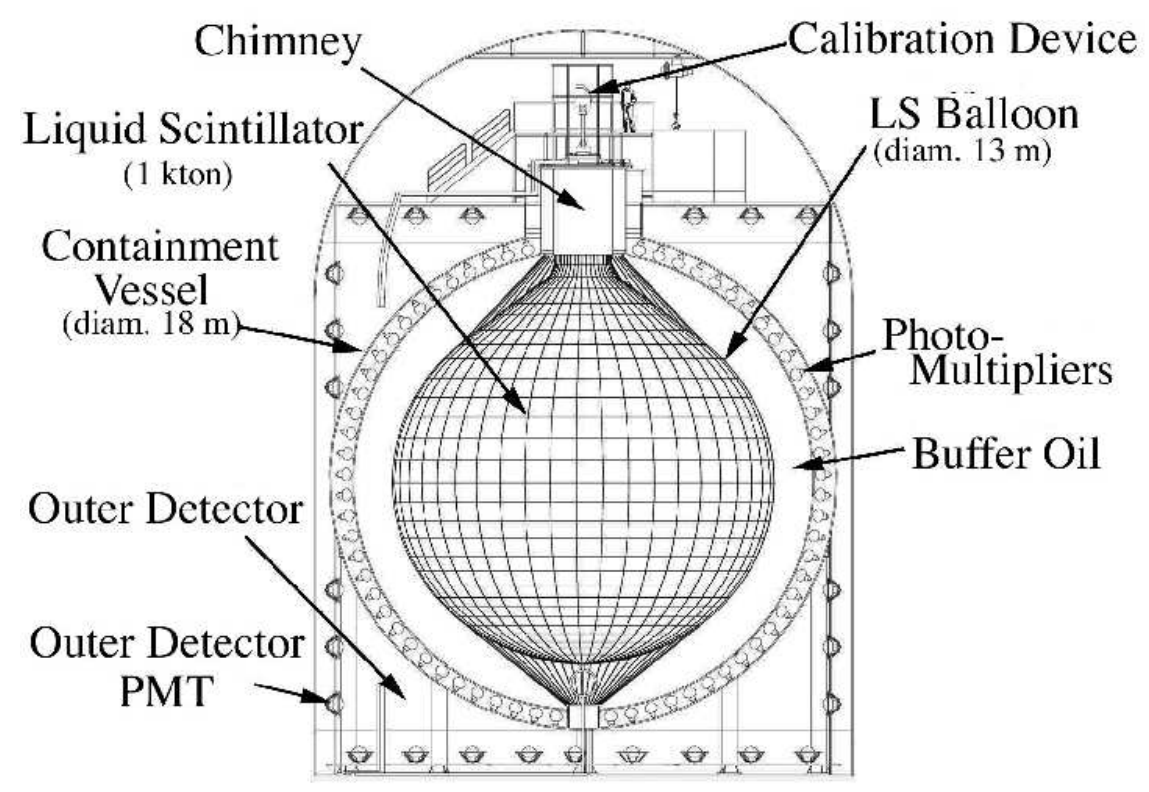

Figure A.1: This diagram from [136] shows a side view of the KamLAND detector.

comes from reactors that are 140 to $210 \mathrm{~km}$ away [135]. The combination of reactor antineutrino energies and distances and the large flux of anti-neutrinos means that KamLAND is sensitive to the same neutrino oscillation parameter space as solar neutrino experiments. The anti-neutrinos are detected in KamLAND via the following reaction

$$
\bar{\nu}_{e}+p \rightarrow e^{+}+n
$$

where the $e^{+}$is detected by scintillation light (in combination with the two $0.511 \mathrm{MeV}$ $\gamma$-rays produced when it annihilates with an $\left.e^{-}\right)$and the neutron is detected through the delayed capture on a proton, yielding a $2.2 \mathrm{MeV} \gamma$-ray.

Unlike in SNO, where the neutrino interactions are not produced in coincidence with another light signal, in KamLAND the study of time correlations is very important. Since the average neutron capture time is on the order of $500 \mu$ sec [135], excellent time 
resolution is necessary and the ability to discriminate between multiple hits in a PMT during a given event is very important. Detailed pulse shape information can also provide $n$ - $\gamma$-ray discrimination. For these reasons, PMT pulses in KamLAND are captured digitally using a waveform recording design developed at LBNL. Approximately 4000 Analog Transient Waveform Digitizer (AWTD) chips are used to digitize the pulses from the photomultiplier tubes (two ATWD chips will be used for each PMT). This chip is a modified version (Version 3.1) of the one originally described in [137]. Within the integrated circuit waveforms are captured and held temporarily in an array of storage capacitors and are then digitized using a Wilkinson multi-ADC. Each of the ATWDs has four channels. In the KamLAND electronics, three of these will are used for PMT pulses (one high gain channel, one medium gain channel, and one low gain channel) and the fourth is be used for timing signals from the master clock. Here we will refer to these channels as H, M, L, and X. Each of the four channels has 128 time bins. The time separation between bins can be tuned from 1 ns to 10 ns using an analog current. Each bin stores a 10 bit digital number. A sample waveform is shown in Figure A.2.

\section{A.2 ATWD Tester Setup}

Due to the complexity of the ATWD circuit design, approximately 8000 chips were fabricated and it was necessary to test these for functionality. A testing board was fabricated from an existing 12-channel KamLAND prototype data acquisition board. The board was modified to power the ATWDs from an external source (to monitor the current consumption) and to include a computer-controlled pulser. The pulser could provide both very 


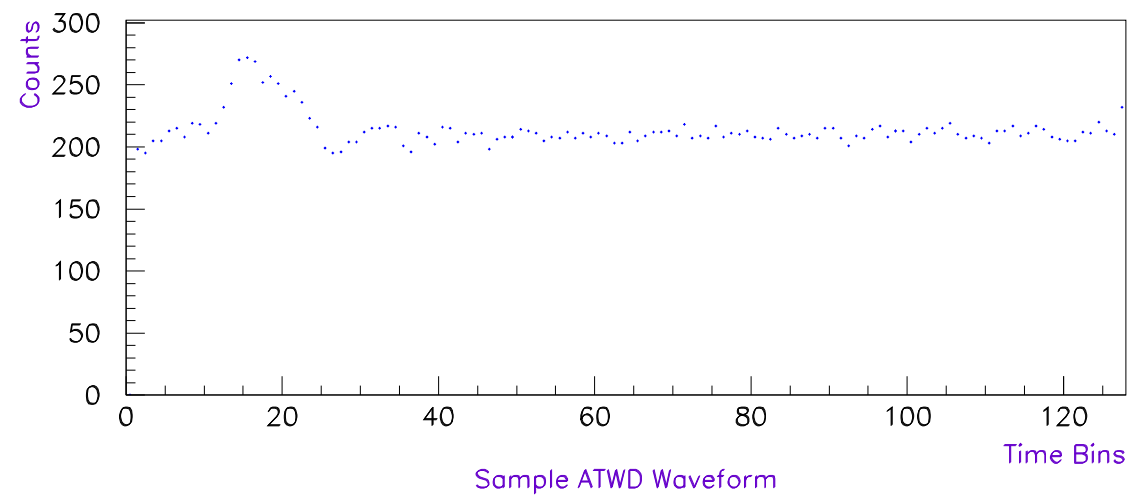

Figure A.2: This plot shows the ATWD output for a sample PMT pulse.

broad pulses (which would cover the entire ATWD digitization time) and high frequency pulses (approximately $20 \mathrm{MHz}$ ).

During the testing, the signal input to the ATWD chip consisted of $2.61 \mathrm{~V}$ pedestal with negative-going pulses added to the pedestal. (So, for example a pulse of amplitude $0.5 \mathrm{~V}$ starts at $2.61 \mathrm{~V}$ and goes down to $2.11 \mathrm{~V}$.) In the initial testing, it was found that all chips are linear down to an input voltage of approximately $740 \mathrm{mV}$. Below $740 \mathrm{mV}$, the chips become non-linear and will tend to output the maximum value of 1023 counts. In the initial testing phase on approximately 50 chips the gain variations between the chips were also studied and were found to be roughly $2 \%$.

\section{A.3 Testing Criteria and Procedure}

The testing software was written by Sanshiro Enomoto using the Kinoko package. For the mass testing it was desired to study the power consumption, pedestal shape, lin- 
earity, gain, time response, and cross talk between channel for all of the ATWDs. These tests were applied to all four channels. In the testing program, data was collected and the chips needed to pass the 10 criteria given below. Note that these tests were applied in the order given here. A detailed description of the testing procedure and the testing software was given in [138].

1) Bad Current: If the current consumed by the chip was less than $20 \mathrm{~mA}$ or greater than $30 \mathrm{~mA}$ the chip was rejected.

2) Did not Digitize: If the chip did not respond within 1 second, it was rejected.

3) Bad Deviation Across Waveform during Pedestal Test: For the pedestal check, no pulser signals were applied to the chip. Approximately 50 pedestal waveforms were obtained. The mean and deviation across the entire waveform was obtained. It the deviation was greater than 7 counts, the chip was rejected. The helped to catch chips with stuck bits. It was noted that the last three bins normally tended to have high pedestal, so they were not included. The pedestals were normally approximately 120 counts. The high gain channel (which was the first one read out) normally had a slightly lower pedestal than the other channels.

4) Bad Deviation in a Bin During Pedestal Test: The same 50 waveforms discussed above were also analyzed to find the mean and deviation on each of the 128 time bins. If the deviation on any one bin was greater than 2 counts, the chip was rejected.

5) Samples Reached 0 or 1023: Throughout the pedestal and linearity tests, if any waveform had 3 or more bins with the minimum (0) or maximum (1023) count values the chip was rejected. This was a sign of non-linearity. 
6) Bad Linearity Slope: For the linearity test, square pulses of height $0 \mathrm{~V}, .48$ $\mathrm{V}, .72 \mathrm{~V}, .96 \mathrm{~V}, 1.2 \mathrm{~V}, 1.44 \mathrm{~V}, 1.675 \mathrm{~V}$. , and $1.87 \mathrm{~V}$ were applied to the chips. (These pulses were negative going and were added to the pedestal value of $2.61 \mathrm{~V}$, i.e. the $0.48 \mathrm{~V}$ pulse had a max value of 2.61 and went down to $2.13 \mathrm{~V}$.) These pulses were very broad and covered the entire ATWD acquisition time. The mean and deviation of these pulses was taken across the 128 bins. Since the chips generally tended to be linear in the middle of the voltage range, the mean values of the five middle pulses $(.48 \mathrm{~V}$ to $1.675 \mathrm{~V})$ were fit to a line. To provide a check of the chip gain, any chip with a slope (measured in counts per volt) less than 345 or greater than 430 was rejected.

7) Bad RMS across waveform during linearity test: As mentioned above, the deviation for each of the 7 pulses in the linearity test was taken. If the deviation was large, it might indicate a stuck bit or non-linearity. The output values tended to become very jumpy as a chip was beginning to behave non-linearly. Any chip with a deviation greater than 10 counts was rejected.

8) Bad Linearity Extrapolation: As mentioned above, only the five middle pulses were fit to a line. This line was then extrapolated to $0 \mathrm{~V}$ and $1.87 \mathrm{~V}$. If either of the measured values for these voltages deviated from the extrapolation by more than 10 counts, the chip was rejected as it was becoming non-linear.

9) Bad timing Chi-Squared or Gain: For the timing check, a high frequency $(20 \mathrm{MHz})$ square pulse was fed to the chip. The edges of these pulses were then found and the edge number was plotted as a function of the time bin location for that edge. For a uniform timing response, we would expect a straight line for this plot. The data were fit 
to a straight line. If the Chi-squared of this fit was greater than .5, the chip was rejected. Also, to insure that the chips all had similar timing gains, if the slope of the line was greater than 18 or less than 15.5 , the chip was rejected.

10) High Crosstalk: One last concern was cross talk between the four channels. For this measurement, a $20 \mathrm{MHz}$ pulse was applied to only one of the channels. The waves collected on the other three channels were then compared to the pedestals taken earlier in the testing process. If any bin changed by more than 30 counts, the chip was rejected. This was repeated for all four channels.

If a chip passed all of the above criteria, it was considered to be acceptable. Rejected chips were sorted according to the first criteria that they failed, and saved for future reference.

\section{A.4 Testing Results}

Two different batches of silicon were tested, one from June 2000 and one from April 2001. The June batch contained 724 chips which were tested with 581 of these chips passing. The April 2001 batch had 7744 chips tested, with 6117 passing the test criteria. It was noted that the two batches had slightly different linearity and timing gains. Therefore, only chips from the April 2001 were used in the KamLAND electronics. The result are shown in the Table A.1.

These results show that the total fraction of chips which passed the testing criteria was $80.2 \%$ for the June 2000 batch and $79.0 \%$ for the April 2001 batch. The most common failure modes were a bad RMS across the 128 channels for the pedestals and a bad current 
Table A.1: The table below shows the number of chips for each of the 10 failure modes. Recall that the failure mode shown here is only the first criteria which the chip failed on. This table contains 143 failed chips from the June 2000 chip batch and 1627 failed chips from the April 2001 batch.

\begin{tabular}{|c|c|}
\hline \hline Failure Mode & Number Failed \\
\hline \hline 1 & 455 \\
\hline 2 & 119 \\
\hline 3 & 606 \\
\hline 4 & 252 \\
\hline 5 & 103 \\
\hline 6 & 65 \\
\hline 7 & 124 \\
\hline 8 & 29 \\
\hline 9 & 14 \\
\hline \hline 10 & 3 \\
\hline \hline Total & 1770 \\
\hline
\end{tabular}

value. 


\section{Bibliography}

[1] D. Griffiths. Introduction to Elementary Particles. John Wiley and Sons, Inc., New York, 1987.

[2] L. M. Brown. The idea of the neutrino. Physics Today, pages 23-28, September 1978.

[3] E. Fermi. Tentativo di una teoria dei raggi $\beta$. Il Nuovo Cimento, Series 8, 11(1):1-19, 1934.

[4] E. Fermi. Zeitschrift fur Physik, 88:161, 1934.

[5] E. Fermi and F. L. Wilson (translator). Fermi's theory of beta decay. Amer. J. Phys., 36:1150, 1968.

[6] H. R. Crane and J. Halpern. New experimental evidence for the existence of a neutrino. Phys. Rev., 53:789-794, 1938.

[7] J. S. Allen. Experimental evidence for the existence of a neutrino. Phys. Rev., 61:692697, 1942.

[8] R. F. Christy et al. The conservation of momentum in the disintegration of $\mathrm{Li}^{8}$. Phys. Rev., 72(8):698-711, 1947. 
[9] C. L. Cowan, Jr., F. Reines, et al. Detection of the free neutrino: A confirmation. Science, 124(3212):103, 1956.

[10] R. Davis, Jr. An attempt to detect the neutrinos from a nuclear reactor by the $\mathrm{Cl}^{37}\left(\bar{\nu}, e^{-}\right) \mathrm{Ar}^{37}$ reaction. Bull. of Amer. Phys. Soc., Series 2, 1(4):219, 1956.

[11] C. S. Wu et al. Experimental test of parity conservation in $\beta$ decay. Phys. Rev., 105:1413-1415, 1957.

[12] T. D. Lee and C. N. Yang. Parity nonconservation and a two-component theory of the neutrino. Phys. Rev., 105:1671-1675, 1957.

[13] M. Goldhaber et al. Helicity of the neutrino. Phys. Rev., 109:1015-1017, 1958.

[14] G. Danby et al. Observation of high-energy neutrino reactions and the existence of two-kinds of neutrinos. Phys. Rev. Lett., 9(1):36-44, 1962.

[15] M. Perl et al. Evidence for anomalous lepton production in $\mathrm{e}^{+} \mathrm{e}^{-}$annihilation. Phys. Rev. Lett., 35:1489-1492, 1975.

[16] K. Kodama et al. Observation of tau neutrino interactions. Phys. Lett. B, 504:218$224,2001$.

[17] G. Kane. Modern Elementary Particle Physics: the fundamental particles and forces? Addison-Wesley, Reading, Massachusetts, updated paperback edition edition, 1993.

[18] Particle Data Group. Review of Particle Physics. Phys. Rev D, 66, 2002.

[19] U. S. Department of Agriculture, Agricultural Research Service. USDA Nutrient Database for Standard Reference, Release 15, 2002. 
[20] F. W. Walker and J. R. Parrington. Nuclides and Isotropes, Fourteenth Edition. General Electric Company, 1989.

[21] A. Burrows. The birth of neutron stars and black holes. Physics Today, 40:28-37, September 1987.

[22] C. Hagmann. Cosmic neutrinos and their detection. In Proceedings of the APS Meeting, Division of Particles and Fields, Los Angeles, Jan 5-9, 1999, 1999. astro$\mathrm{ph} / 9905258$.

[23] J. N. Bahcall. Neutrino Astrophysics. Cambridge University Press, Cambridge, 1989.

[24] J. N. Bahcall. Solar neutrinos: Where we are, where we are going. Astrophy. J., $467: 475,1996$.

[25] F. H. Shu. The Physical Universe. University Science Books, Sausalito, California, 1982.

[26] W. A. Fowler. Completion of the proton-proton reaction chain and the possibility of energetic neutrino emission by hot stars. Astrophy. J., 127:551-556, 1958.

[27] J. N. Bahcall, W. A. Fowler, et al. Solar neutrino flux. Astrophy. J., 137:344-346, 1963.

[28] R. Davis, Jr. Solar neutrinos II. experimental. Phys. Rev. Lett., 12:305-305, 1964.

[29] R. Davis, Jr. et al. Search for neutrinos from the Sun. Phys. Rev. Lett., 20:1205-1209, 1968. 
[30] B. Cleveland et al. Measurement of the solar electron neutrino flux with the Homestake chlorine detector. Astrophy. J., 496:505-526, 1998.

[31] J. N. Bahcall et al. How uncertain are solar neutrino predictions? Phys. Lett. B, 433:1-8, 1998.

[32] J. N. Abdurashitov et al. Measurement of the solar neutrino capture rate with gallium metal. Phys. Rev. C, 60:055801, 1999.

[33] M. Altmann et al. GNO solar neutrino observations: Results for GNO I. Phys. Lett. B., 490:16-26, 2000.

[34] The SNO Collaboration. Sudbury Neutrino Observatory Proposal, October 1987.

[35] Y. Fukuda et al. Solar neutrino data covering solar cycle 22. Phys. Rev. Lett., 77(9):1683-1686, 1996.

[36] S. Fukuda et al. Determination of solar neutrino oscillation parameters using 1496 days of Super-Kamiokande-I data. Phys. Lett. B, 539:179-187, 2002.

[37] B. Kayser et al. The Physics of Massive Neutrinos. World Scientific, Singapore, 1989.

[38] B. Kayser. On the quantum mechanics of neutrino oscillations. Phys. Rev. D, $24(1): 110-116,1981$.

[39] L. Wolfenstein. Neutrino oscillations in matter. Phys. Rev. D, 17:2369-2374, 1978.

[40] S. P. Mikheyev and A. Y. Smirnov. Resonance enhancement of oscillations in matter and solar neutrino spectroscopy. Sov. Jour. Nucl. Phys., 42:913-917, 1985. 
[41] A. Gouvêa et al. The dark side of the solar neutrino parameter space. Phys. Lett. B, 490:125-130, 2000.

[42] J. N. Bahcall et al. Solar neutrinos: global analysis and implications for SNO. JHEP, 05:015, 2001.

[43] Y. Fukuda et al. Evidence for oscillation of atmospheric neutrinos. Phys. Rev. Lett, 81:1562-1567, 1998.

[44] T. Araki et al. Measurement of neutrino oscillations with KamLAND: Evidence of spectral distortion. Sumitted to Phys. Rev. Lett., June 2004. hep-ex/0406035.

[45] C. Athanassopoulos et al. Results on $\nu_{\mu} \rightarrow \nu_{e}$ neutrino oscillations from the LSND experiment. Phys. Rev. Lett, 81:1774-1777, 1998.

[46] C. Athanassopoulos et al. Evidence for neutrino oscillations from muon decay at rest. Phys. Rev. C, 54:2685-2708, 1996.

[47] The BooNE Collaboration. A proposal for an experiment to measure $\nu_{\mu} \rightarrow \nu_{e}$ oscillations and $\nu_{\mu}$ disappearance at the Fermilab Booster: BooNE, December 1997.

[48] S. Raby and R. Slansky. Neutrino masses: How to add them to the standard model. Los Alamos Science, 25:64-70, 1997.

[49] H. H. Chen. Direct approach to resolve the solar-neutrino problem. Phys. Rev. Lett., 55:1534-1536, 1985.

[50] J. Boger et al. The Sudbury Neutrino Observatory. Nucl. Inst. and Meth. A, 449:172$207,2000$. 
[51] G. T. Ewan et al. The Sudbury Neutrino Observatory. Nucl. Inst. and Meth. A, 314:372-379, 1992.

[52] X. Dai et al. Multi-elemental and spectroscopic analyses of the desalination samples. SNO internal note, Jan. 2004.

[53] G. Doucas et al. Light concentrators for the Sudbury Neutrino Observatory. Nucl. Inst. and Meth. A, 370:579-596, 1996.

[54] S. D. Biller et al. Measurements of photomultiplier single photon counting efficiency for the Sudbury Neutrino Observatory. Nucl. Inst. and Meth. A, 432:364-373, 1999.

[55] C. J. Jillings et al. The photomultiplier tube testing facility for the Sudbury Neutrino Observatory. Nucl. Inst. and Meth. A, 373:421-429, 1996.

[56] K. T. Lesko C. Okada and A. W. P. Poon. Determination of true north for the Sudbury Neutrino Observatory detector. Technical Report SNO-STR-2000-015, June 2000.

[57] D. Cowen et al. The Sudbury Neutrino Observatory electronics chain. IEEE Transactions on Nuclear Science, 42(4):925-932, 1995.

[58] R. J. Ford. Nitrogen/dye laser for the optical calibration of SNO. Master's thesis, Queen's University, Kingston, Ontario, Canada, 1993.

[59] M. R. Dragowsky et al. The ${ }^{16} \mathrm{~N}$ calibration source for the Sudbury Neutrino Observatory. Nucl. Inst. and Meth. A, 481:284-296, 2002. 
[60] R. B. Firestone. Table of Isotopes. John Wiley and Sons, Inc., New York, 8th edition, 1996.

[61] R. R. Spencer et al. A measurement of the average number of prompt neutrons from the spontaneous fission of Californium-252. Nucl. Sci. and Engin., 80:603-629, 1982.

[62] E. B. Norman. ${ }^{241} \mathrm{Am}-{ }^{9}$ Be source for neutron and $\gamma$-ray calibrations of SNO. SNO internal note, February 2002.

[63] S. Croft. The use of neutron intensity calibrated ${ }^{9} \mathrm{Be}(\alpha, \mathrm{n})$ as $4438 \mathrm{keV}$ gamma-ray reference standards. Nucl. Inst. Meth. A, 281:103-116, 1989.

[64] N. Tagg. The ${ }^{8}$ Li calibration source and through-going muon analysis in the Sudbury Neutrino Observatory. PhD thesis, University of Guelph, Guelph, Ontario, Canada, 2001.

[65] A. W. P. Poon et al. A compact ${ }^{3} \mathrm{H}(\mathrm{p}, \gamma){ }^{4} \mathrm{He} 19.8 \mathrm{MeV}$ gamma ray source for energy calibration at the Sudbury Neutrino Observatory. Nucl. Inst. and Meth. A, 452:115$129,2000$.

[66] A. W. P. Poon. Energy calibration of the Sudbury Neutrino Observatory using monoenergetic gamma-ray sources. PhD thesis, University of British Columbia, Vancouver, Canada, 1998.

[67] The SNO Collaboration. The SNOMAN User's Manual, Version 5.0, Feb 2003.

[68] CERN, Geneva, Switzerland. The ZEBRA System, CERN Program Library Long Write-up Q100/Q101. 
[69] CERN, Geneva, Switzerland. GEANT Detector Description and Simulation tool, CERN Program Library Long Write-up Q123.

[70] W. Nelson et al. The EGS4 Code System, SLAC Report 264. SLAC, Palo Alto, California, 1985.

[71] A. Fasso et al. FLUKA92, Proceedings of workshop on Simulating Accelerator Radiation Environments, 11-15 Jan 1993, Santa Fe Los Alamos Report LA-12835-C. Los Alamos National Laboratory, Los Alamos, NM, 1993.

[72] Los Alamos National Laboratory, Los Alamos, NM. MCNP Manual, Los Alamos Report LA-13709-M, 2000.

[73] P. Antonioli et al. A three-dimensional code for muon propagation through the rock: MUSIC. Astroparticle Physics, 7:357-368, 1997.

[74] Naoya Hata. Theoretical Implications of Solar neutrino Observations. PhD thesis, University of Pennsylvania, Philadelphia, Pennsylvania, 1995.

[75] CERN, Geneva, Switzerland. The HBOOK reference manual, CERN Program Library Long Write-up Y250.

[76] CERN, Geneva, Switzerland. PAW Physics Analysis Workstation: An Introductory Tutorial, CERN program library long write-up Q121.

[77] R. Brun and F. Rademakers. ROOT - an object oriented data analysis framework. Nucl. Inst. Meth. A, 389:81-86, 1999. See also http://root.cern.ch. 
[78] Q. R. Ahmad et al. Measurement of the rate of $\nu_{e}+d \rightarrow p+p+e^{-}$interactions produced by ${ }^{8} B$ solar neutrinos at the Sudbury Neutrino Observatory. Phys. Rev. Lett, 87:071301-1-071301-6, 2001.

[79] Q. R. Ahmad et al. Direct evidence for neutrino flavor transformation from the neutral-current interactions in the Sudbury Neutrino Observatory. Phys. Rev. Lett, 89:011301-1-011301-6, 2002.

[80] J. N. Bahcall et al. Solar models: Current epoch and time dependencies, neutrinos, and helioseismological properties. Astrophys. Jour., 555:990-1012, July 2001.

[81] International Commission on Radiation Units and Measurements, Bethesda, MD. Stopping powers for electrons and positrons, report 37 edition, 1984.

[82] M. E. Moorhead. Grid fitter for reducing tails in spatial distributions. Technical Report SNO-STR-95-042, August 1996.

[83] X. Chen. An improved grid fitter. Technical Report SNO-STR-2000-004, April 2000.

[84] K. S. Krane. Introductory Nuclear Physics. John Wiley and Sons, New York, 1988.

[85] A. Bellerive et al. Energy and optics measurement for the salt phase of SNO. SNO internal note, May 2003.

[86] M. R. Dragowsky et al. Measurement of the ${ }^{252} \mathrm{Cf}$ acrylic source standard. Technical Report SNO-STR-2001-009, November 2001.

[87] G. Knoll. Radiation Detection and Measurement. John Wiley and Sons, Inc., New York, 1989. 
[88] B. Krusche et al. Gamma ray energies and ${ }^{36} \mathrm{Cl}$ level scheme from the reaction ${ }^{35} \mathrm{Cl}(\mathrm{n}, \gamma)$. Nucl. Phys. A, 386:245-268, 1982.

[89] M. Holmberg. The (n,2n) cross section of ${ }^{2} \mathrm{H}$ in the energy region 4.0-6.5 MeV. Nucl. Phys. A, 129:327-330, 1969.

[90] M. L. Rustgi et al. Two-body effects and deuteron photodisintegration with polarized and unpolarized gamma rays. Phys. Rev. C., 29:785-793, 1984.

[91] M. Kos et al. Neutron detection efficiency during the salt phase of SNO. SNO internal note, June 2003.

[92] M. Kos. Neutron detection efficiency for the complete salt data set. SNO internal note, June 2004.

[93] N. McCauley. PhD thesis, Oxford University, 2002.

[94] S. Brice et al. Sacrifice and contamination in SNO data cleaning cuts. SNO internal note, Nov 2000.

[95] M. Dunford et al. Updates to data cleaning for the salt phase. SNO internal note, Aug 2003.

[96] SNO Collaboration. SNO preliminary analysis for neutral current rate measurement. SNO internal note, April 2002.

[97] J. Farine et al. Final ex-situ and merged pd in $\mathrm{D}_{2} \mathrm{O}$. SNO internal note, May 2004. http://manhattan.sno.laurentian.ca/sno/ananoteb.nsf/URL/MANN-5YYGNT. 
[98] J. Farine et al. Final merged Th/U concentrations in $\mathrm{H}_{2} \mathrm{O}$. SNO internal note, May 2004. http://manhattan.sno.laurentian.ca/sno/ananoteb.nsf/URL/MANN-5ZEQFN.

[99] A. W. P. Poon et al. Final in-situ and ex-situ results. SNO internal note, August 2003. http://manhattan.sno.laurentian.ca/sno/ananoteb.nsf/URL/MANN-5QTK3R.

[100] P. Skensved and B. C. Robertson. Summary of backgrounds in SNO. Technical Report SNO-STR-1994-013, 1994.

[101] A. Hallin. Hot acrylic source review summary, March 2001. http://sno.phy.queensu.ca/ hallin/private/HotAcrylicsource/has2.html.

[102] R. K. Heaton et al. $\alpha$-particle induced high-energy $\gamma$-ray yields from light elements. Nuc. Inst. Meth. A, page 317, 1995.

[103] Y.-F. Wang et al. Predicting neutron production from cosmic-ray muons. Phys. Rev. D., 64 .

[104] H. Ishino. Measurement of the solar neutrino energy spectrum at Super-Kamiokande. PhD thesis, University of Tokyo, Jan 1999.

[105] B. Heisinger et al. Production of selected cosmogenic radionuclides by muons 1. Fast muons. Earth and Planetary Science Letters, 200:345-355, 2002.

[106] Y. Koshio. Study of Solar Neutrinos at Super Kamiokande. PhD thesis, University of Tokyo, April 1998.

[107] J. Blatt and V. Weisskopf. Theoretical Nuclear Physics. John Wiley and Sons, New York, 1952. 
[108] Q. R. Ahmad. Muon Correlated Background at the Sudbury Neutrino Observatory. PhD thesis, Brown University, May 2002.

[109] M. Boulay et al. "Other" background sources in the salt phase of SNO. SNO analysis note, August 2003.

[110] G. MacGregor. PhD thesis, Merton College, Oxford University, 2002.

[111] M. Boulay et al. "Other" background sources for the extended salt data. SNO analysis note, April 2004.

[112] C. F. Williamson. Absolute cross sections of the reactions $\mathrm{Na}^{23}(\mathrm{n}, \mathrm{p}) \mathrm{Ne}^{23}$ and $\mathrm{Na}^{23}(\mathrm{n}, \alpha) \mathrm{F}^{20}$. Phys. Rev., 122(6), 1961.

[113] S. C. Mathur and I. L. Morgan. The disintegration of Ar by 13.0-20.2 MeV neutrons. Nucl. Phys., 75, 1966.

[114] R. W. Stooksberry DeJuren, J. A. and M. Wallis. Measurement of the ${ }^{16} \mathrm{O}(\mathrm{n}, \mathrm{p}) \mathrm{N}^{16}$ cross section from 11 to $19 \mathrm{MeV}$. Phys. Rev., 127(4), 1962.

[115] M. Yamada et al. Measurements of the charge and polarization of $1.2 \mathrm{TeV} / \mathrm{c}$ cosmicray muons with the Kamiokande II detector. Phys. Rev. D, 44.

[116] D. F. Measday Suzuki, T. and J. P. Roalsvig. Total nuclear capture rates for negative muons. Phys. Rev. C, 35 .

[117] R. C. Cohen et al. $\mu$ capture in oxygen. Phys. Rev. Lett., 11, 1963.

[118] T. C. Andersen. Light emission from acrylic plastics during fracture. Master's thesis, Laurentian University, 1991. 
[119] S. Oser. Summary of Day-Night backgrounds. SNO analysis note, June 2004.

[120] G. Cowan. Statistical Data Analysis. Oxford University Press, Oxford, UK, 1998.

[121] CERN, Geneva, Switzerland. MINUIT Function Minimization and Error Analysis, CERN Program Library Long Write-up D506.

[122] K. Miknaitis et al. Livetime for the full salt data set. SNO analysis note, May 2004.

[123] R. G. H. Robertson et al. Long $\mathrm{D}_{2} \mathrm{O}$ paper: Target properties. SNO analysis note, May 2003.

[124] P. J. Doe et al. Systematics of the $\mathrm{D}_{2} \mathrm{O}$ target in SNO. Technical Report SNO-STR2002-005, 2002.

[125] M. Butler et al. Neutrino-deuteron scattering in effective field theory at next-to-nextto leading order. Phys. Rev. C, 63:035501, 2001.

[126] J. F. Beacom and S. J. Parke. Normalization of the neutrino-deuteron cross section. Phys. Rev. D, 64:091302, 2001.

[127] S. Nakamura et al. Neutrino reactions on the deuteron. Phys. Rev. C, 63:034617, 2001.

[128] A. Kurylov et al. Radiative corrections in the neutrino-deuterium disintegration. Phys. Rev. C, 65:055501, 2002.

[129] Y. Takeuchi. Cross section correction for salt ES data, 2003. Posted on web site, http://owl.phy.queensu.ca/ takeuchi/private/esrd/. 
[130] J. N. Bahcall and M. Pinsonneault. What do we (not) know theoretically about solar neutrino fluxes? Phys. Rev. Lett., 92(12):121301, March 2004.

[131] Q. R. Ahmad et al. Measurements of day and night neutrino energy spectra at SNO and constraints on neutrino mixing parameters. Phys. Rev. Lett, 89:011302, 2002.

[132] Q. R. Ahmad et al. Measurement of the total active ${ }^{8} \mathrm{~B}$ solar neutrino flux at the Sudbury Neutrino Observatory with enhanced neutral current sensitivity. Phys. Rev. Lett, 92:181301, 2004.

[133] Y. Ashie et al. Evidence for an oscillatory signature in atmospheric neutrino oscillations. Sumitted to Phys. Rev. Lett., April 2004. hep-ex/0404034.

[134] M. Apollonio et al. Search for neutrino oscillations on a long base-line at the CHOOZ nuclear power station. Eur. Phys. Jour. C, 27:331-374, 2003.

[135] U.S. KamLAND Collaboration. Proposal for US participation in KamLAND. KamLAND internal note, May 2000.

[136] K. Eguchi et al. First results from KamLAND: Evidence for reactor antineutrino disappearance. Phys. Rev. Lett, 90:021802-1-021802-4, 2003.

[137] S. Kleinfelder. A multi-gigahertz analog transient recorder integrated circuit. $\mathrm{PhD}$ thesis, University of California, Berkeley, Department of Electrical Engineering and Computer Science, Berkeley, CA, 1992.

[138] A.D. Marino. Testing procedure for analog transient waveform digitizers. KamLAND internal note, May 2000. 